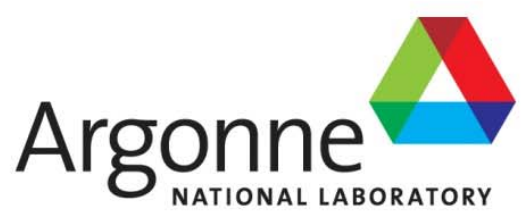

\title{
Use of Wind Power Forecasting in Operational Decisions
}

Decision and Information Sciences Division 


\begin{abstract}
About Argonne National Laboratory
Argonne is a U.S. Department of Energy laboratory managed by UChicago Argonne, LLC under contract DE-AC02-06CH11357. The Laboratory's main facility is outside Chicago, at 9700 South Cass Avenue, Argonne, Illinois 60439. For information about Argonne and its pioneering science and technology programs, see www.anl.gov.
\end{abstract}

\title{
Availability of This Report
}

This report is available, at no cost, at http://www.osti.gov/bridge. It is also available on paper to the U.S. Department of Energy and its contractors, for a processing fee, from:

U.S. Department of Energy

Office of Scientific and Technical Information

P.O. Box 62

Oak Ridge, TN 37831-0062

phone (865) $576-8401$

fax (865) 576-5728

reports@adonis.osti.gov

\section{Disclaimer}

This report was prepared as an account of work sponsored by an agency of the United States Government. Neither the United States Government nor any agency thereof, nor UChicago Argonne, LLC, nor any of their employees or officers, makes any warranty, express or implied, or assumes any legal liability or responsibility for the accuracy, completeness, or usefulness of any information, apparatus, product, or process disclosed, or represents that its use would not infringe privately owned rights. Reference herein to any specific commercial product, process, or service by trade name, trademark, manufacturer, or otherwise, does not necessarily constitute or imply its endorsement, recommendation, or favoring by the United States Government or any agency thereof. The views and opinions of document authors expressed herein do not necessarily state or reflect those of the United States Covernment or any agency thereof, Argonne National Laboratory, or UChicago Argonne, LLC. 


\section{Use of Wind Power Forecasting in Operational Decisions}

by

Audun Botterud, Zhi Zhou, and Jianhui Wang

Decision and Information Sciences Division, Argonne National Laboratory

Ricardo J. Bessa, Hrvoje Keko, Joana Mendes, Jean Sumaili, and Vladimiro Miranda Instituto de Engenharia de Systemas E Computadores do Porto

September 30, 2011 



\section{CONTENTS}

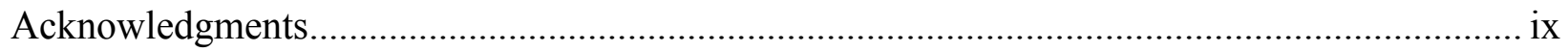

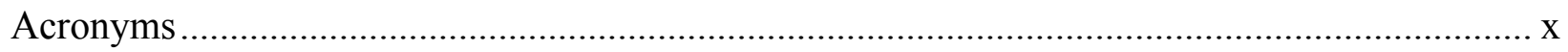

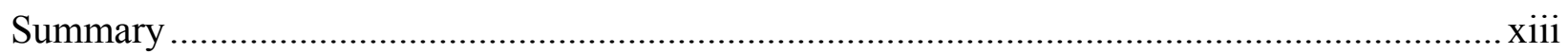

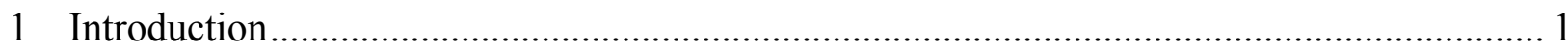

1.1 Wind Power Forecasting ................................................................................... 1

1.1.1 Wind Power Point Forecasting …........................................................ 2

1.1.2 Wind Power Uncertainty Forecasting ........................................................... 2

1.2 Wind Power Forecasting in Operational Decisions …............................................. 3

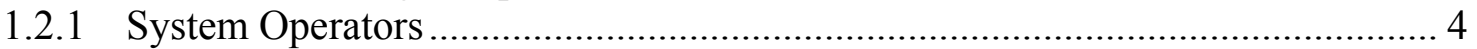

1.2.2 Wind Power Producers ........................................................................... 4

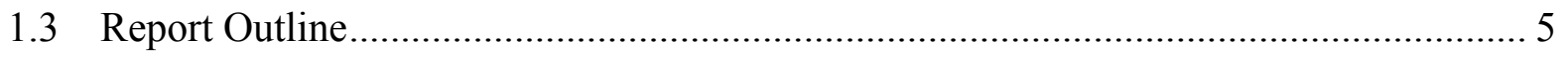

2 Good or Bad Wind Power Forecasting: A Relative Concept .......................................... 7

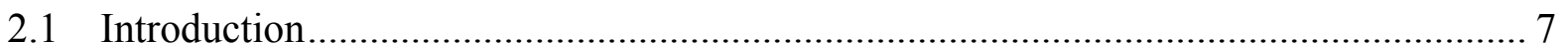

2.2 The "Forecast Consumer Paradigm" ...................................................................... 9

2.2.1 Wind Generation Companies' Viewpoint................................................ 9

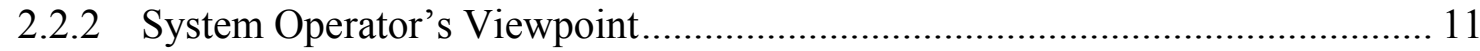

2.2.3 The Compromise Viewpoint, or the Search for Utopia ................................. 13

2.3 Training W2P Models ....................................................................................... 14

2.3.1 Aspects of Training W2P Models ................................................................ 14

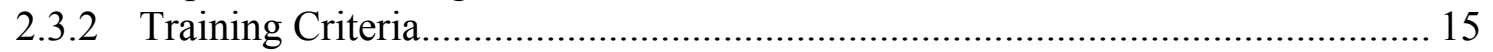

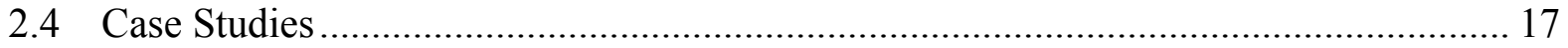

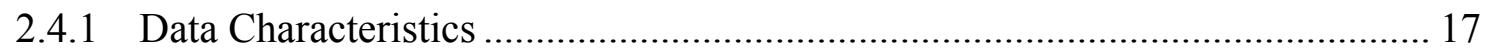

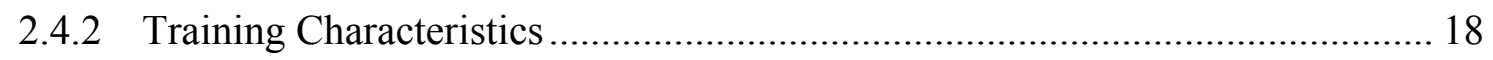

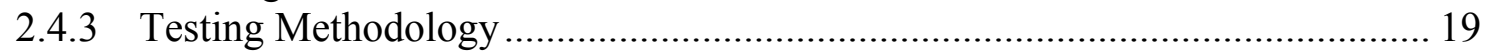

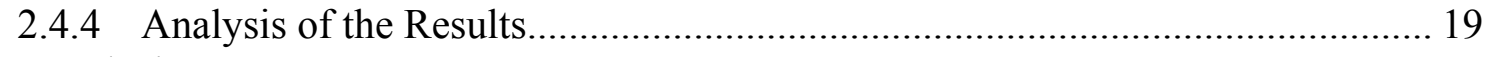

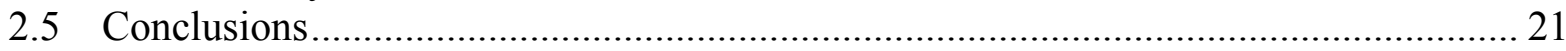

3 Wind Power Forecasting in U.S. Electricity Markets ................................................ 23

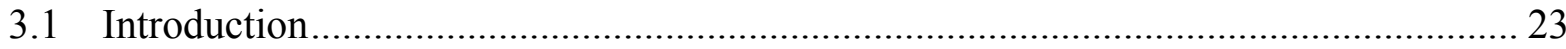

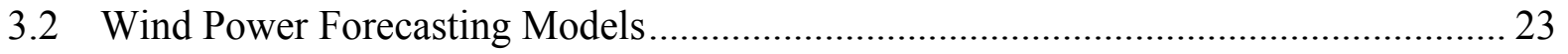

3.3 Use of Forecasting in Market Operations .............................................................. 24

3.3.1 Operating Reserve Requirements ......................................................... 25

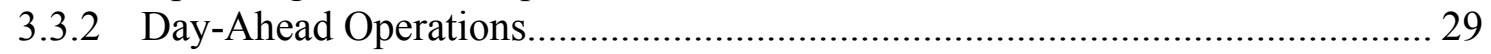

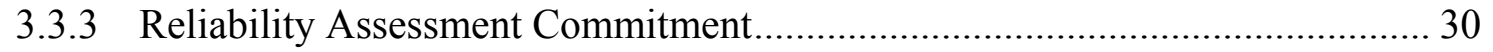

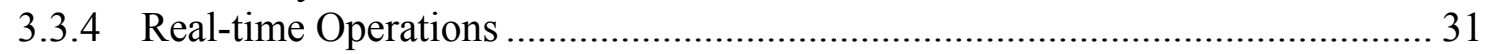

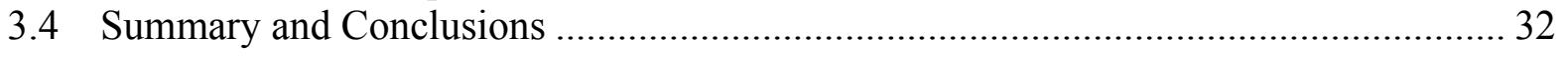




\section{CONTENTS (CONT.)}

4 Wind Power Forecasting Uncertainty and Unit Commitment ........................................ 35

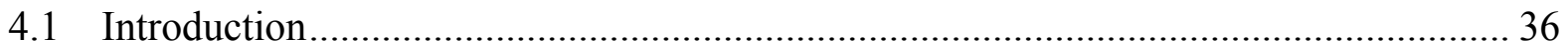

4.2 Forecasting and Scenario Generation Approaches .................................................. 38

4.3 Unit Commitment and Dispatch Formulations …................................................ 39

4.3.1 Objective Function................................................................................. 39

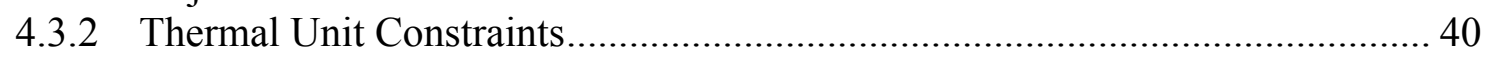

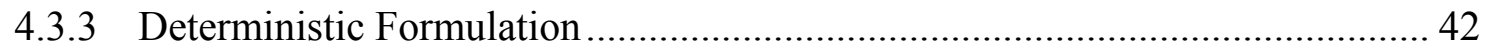

4.3.4 Economic Dispatch ....................................................................... 42

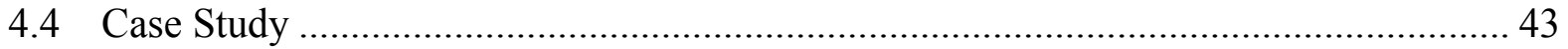

4.4.1 Assumptions.................................................................................... 43

4.4.2 Wind Power and Forecast Characteristics .................................................... 44

4.4.3 Simulated Cases .................................................................................. 45

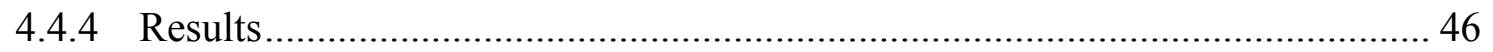

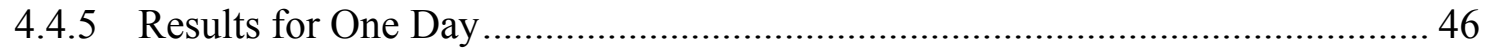

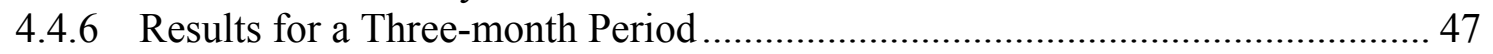

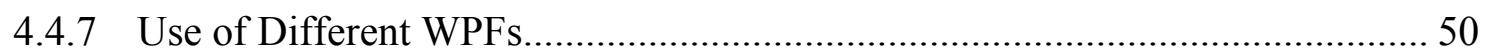

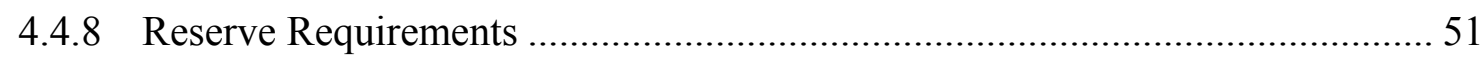

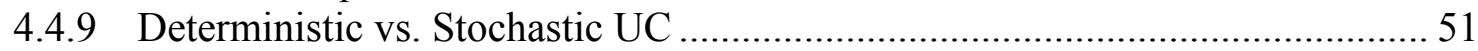

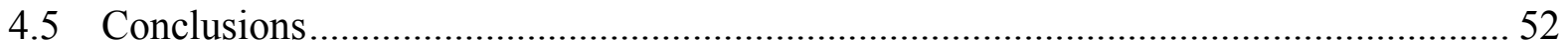

5 Representative Wind Power Scenarios and their Probabilities for Stochastic Models.......... 55

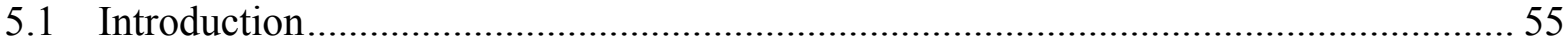

5.2 Scenario Generation and the Need for Scenario Reduction....................................... 57

5.3 Wind Power Scenario Clustering and Modes Finding............................................. 57

5.3.1 Application of Classical Clustering Techniques to Wind Power Scenarios ...... 57

5.3.2 Modes Finding using the ITL Mean Shift Algorithm .................................. 58

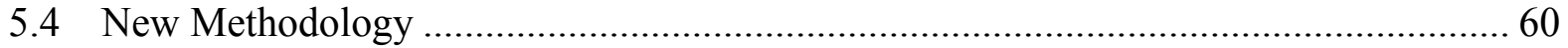

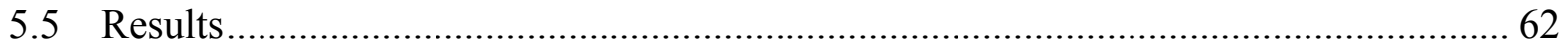

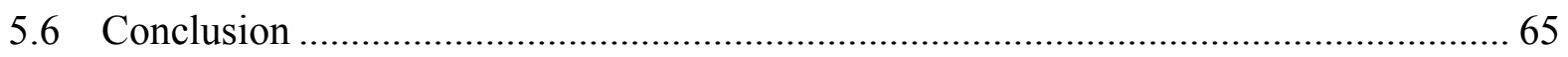

6 Unit Commitment and Operating Reserves with Probabilistic Wind Power Forecasts.......... 67

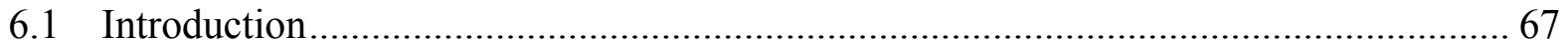

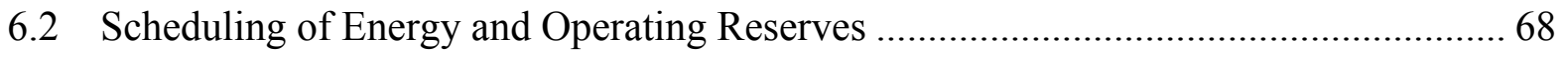

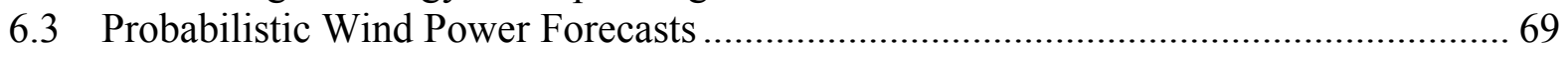

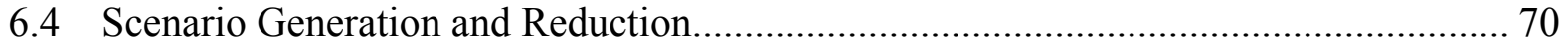

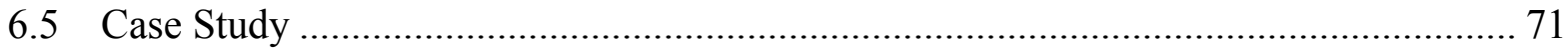

6.5.1 Probabilistic Wind Power Forecasts ....................................................... 71

6.5.2 Overview of Simulated Cases ................................................................... 75

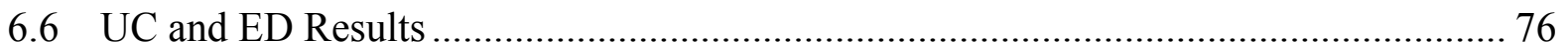

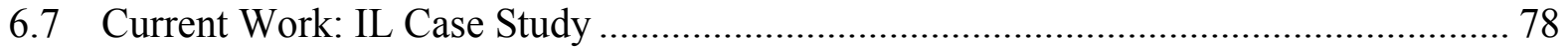

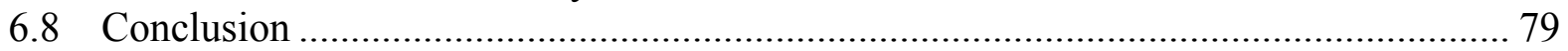




\section{CONTENTS (CONT.)}

7 Wind Power Trading Under Uncertainty in Electricity Markets .................................... 81

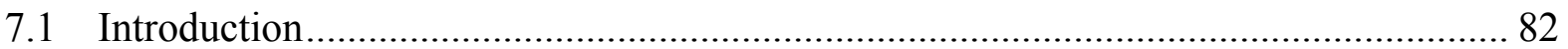

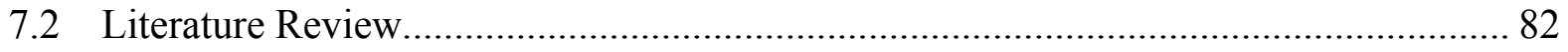

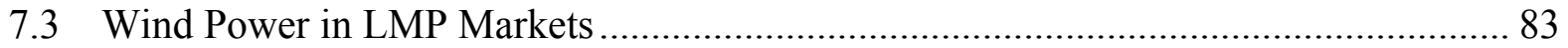

7.4 Probabilistic Wind Power Forecasting.................................................................. 87

7.5 A Model for Wind Power Trading under Uncertainty............................................. 89

7.5.1 Representation of Uncertainty in DA and RT LMPs.................................. 90

7.5.2 Mathematical Formulation of the DA Bidding Problem ................................. 90

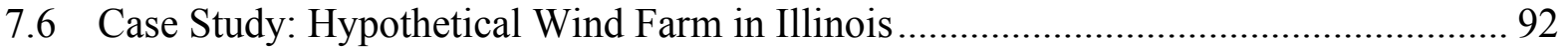

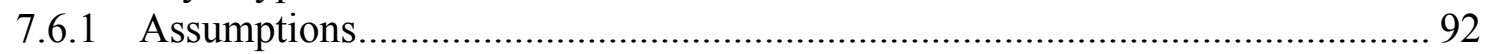

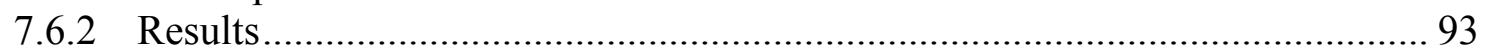

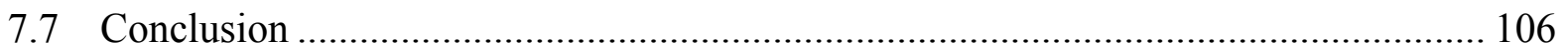

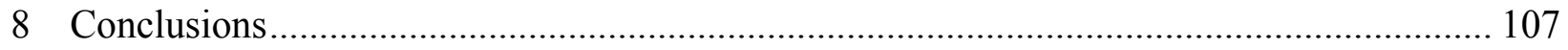

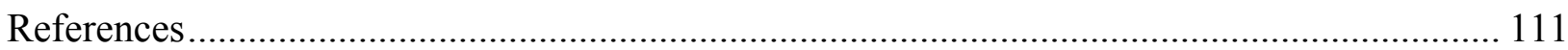

\section{LIST OF FIGURES}

Fig. 1-1 Illustration of WPF system............................................................................... 2

Fig. 2-1 Basic arrangement of a W2P identifying its three main modules. .............................. 15

Fig. 3-1 Uncertainty representation in wind power forecasts: (a) probabilistically by a set of intervals or quantiles (left), and (b) scenarios of wind power generation representing the temporal dependency of forecast errors (right).............................. 24

Fig. 3-2 Market operations timeline for MISO, indicating where WPF could play

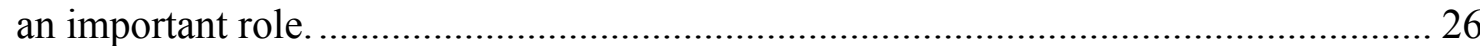

Fig. 3-3 Traditional operating reserve categories as defined by NERC and a proposed new category to address increased uncertainty and variability

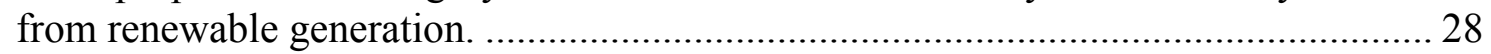

Fig. 3-4 Role of WPF in electricity market operations......................................................... 33

Fig. 4-1 Wind power forecast (deterministic point forecast and 10 stochastic scenarios)

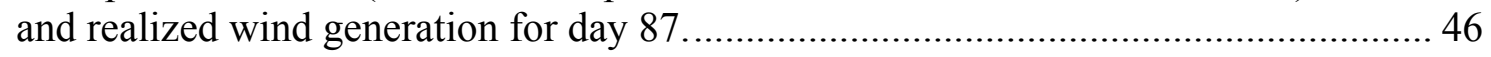

Fig. 4-2 Number of on-line units for day 87 ..................................................................... 48

Fig. 4-3 Available operating reserves in RT dispatch.................................................... 48

Fig. 4-4 Total hours of commitment for thermal units in all cases.......................................... 48

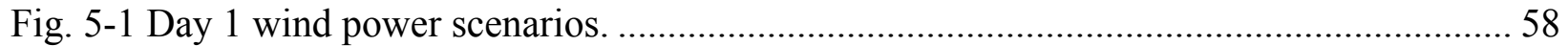

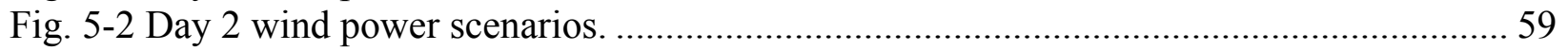

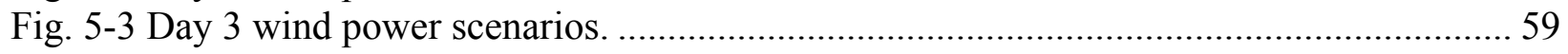

Fig. 5-4 Day 3 Mode finding results for the three-day set of scenarios - thick lines represent the modes discovered, coherent with the merged sets. ............................. 60

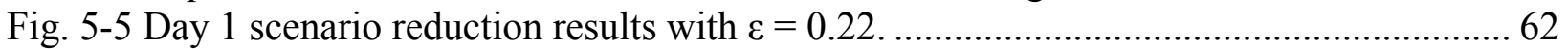




\section{LIST OF FIGURES (CONT.)}

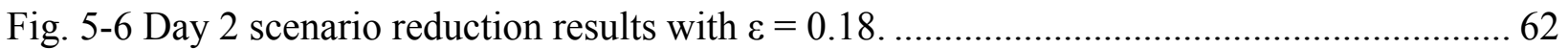

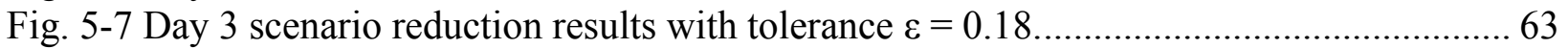

Fig. 5-8 Day 1 distribution of the maximum deviation between each pair of 2 scenarios. ......... 64

Fig. 5-9 Day 2 distribution of the maximum deviation between each pair of 2 scenarios. ......... 64

Fig. 5-10 Day 3 distribution of the maximum deviation between each pair of 2 scenarios. ........ 64

Fig. 6-1 Probabilistic forecasts of wind power quantiles $(5 \%, 10 \%, \ldots 95 \%)$ based on

QR and realized wind power generation (dotted line) for day 1 ................................ 72

Fig. 6-2 Calibration diagram for QR and NW methods, Oct.-Dec. 2006. ............................... 72

Fig. 6-3 Averages of 10 scenarios with reduction methods SR1, SR2, and SR3 as compared to the average of the original 1,000 scenarios, QR, and days 1 and 2 .

Fig. 6-4 Variances for 10 scenarios with reduction methods SR1, SR2, and SR3 as compared to the average of the original 1,000 scenarios, $\mathrm{QR}$, and days 1 and 2 .

Fig. 6-5 Variances for 100 scenarios with reduction methods SR1, SR2, and SR3 as compared to the average of the original 1000 scenarios, QR, and days 1 and 2 .....

Fig. 6-6 Overview of simulated total costs for deterministic cases. ...................................... 76

Fig. 6-7 Overview of simulated total costs for stochastic cases. ........................................... 76

Fig. 6-8 Simulation of two settlement electricity markets in Illinois case study...................... 78

Fig. 7-1 Examples of hourly financial settlements as function of deviation between RT delivery and DA schedule with penalties: pen $_{\mathrm{DA}}=0$ (left), and penDA $=5 \$ / \mathrm{MWh}$ (right), and LMP differences: $p R T, h m-p D A$, $h m=3,-3,0 \$$ MWh (upper, middle, lower)................................................... 87

Fig. 7-2 Probabilistic representation of wind power forecast as intervals or quantiles.............. 88

Fig. 7-3 Forecast scenarios of wind power generation. ..................................................... 88

Fig. 7-4 Excerpts from Excel interface. ................................................................ 91

Fig. 7-5 Wind power forecast: Deterministic point forecast (solid thick line) and 10 forecast scenarios for selected day (Oct. 9, 2006). ....................................... 92

Fig. 7-6 Estimated DA and RT price parameters for selected day (Oct. 9, 2006)................... 93

Fig. 7-7 30 scenarios for DA (upper) and RT (lower) prices

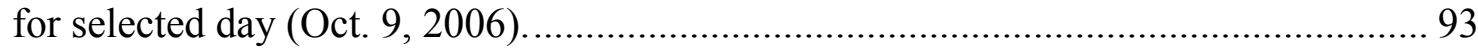

Fig. 7-8 Expected profit (E) and CVAR criteria (C) as function of DA bid quantity, no deviation penalty (hour 5, Oct. 9, 2006).

Fig. 7-9 Expected utility for the risk-averse $(\beta=-3)$ and risk-prone $(\beta=3)$ decision maker, no deviation penalty.

Fig. 7-10 Variance and conditional value at risk, no deviation penalty (hour 5, Oct. 9, 2006). *Without wind power curtailment during negative prices.

Fig. 7-11 Illustration of the trade-off between expected profit and conditional value at risk, no deviation penalty, (hour 5, Oct. 9, 2006).................................... 96

Fig. 7-12 Illustration of the trade-off between expected profit and conditional value at risk, \$5/MWh deviation penalty, (hour 5, Oct. 9, 2006)... 


\section{LIST OF FIGURES (CONT.)}

Fig. 7-13 Optimal DA bidding under different decision criteria for 24 hours

(Oct. 9, 2006). No deviation penalty. pf is the point forecast. 98

Fig. 7-14 Optimal DA bidding under different decision criteria for 24 hours

(Oct. 9, 2006). US\$5/MWh penalty. pf is the point forecast. 98

Fig. 7-15 Optimal DA bidding under different decision criteria for 24 hours

(Oct. 9, 2006). US\$10/MWh penalty. pf is the point forecast. 99

Fig. 7-16 Optimal DA bidding with risk prone decision criteria $(\beta=3)$ for different deviation penalties $(0,5,10 \$ / \mathrm{MWh})$. pf is the point forecast. (Oct. 9, 2006).

Fig. 7-17 Illustration of the trade-off between expected daily profit and conditional value at risk, no deviation penalty (Oct. 9, 2006).

Fig. 7-18 Illustration of the trade-off between expected daily profit and conditional value at risk, \$5/MWh deviation penalty (Oct. 9, 2006).

Fig. 7-19 Total October profit vs. hourly conditional value at risk, no deviation penalty. ........ 101

Fig. 7-20 Total October profit vs. average absolute deviation from schedule, no deviation penalty.

Fig. 7-21 Total monthly profit vs. hourly conditional value at risk, $\$ 5 / \mathrm{MWh}$ deviation penalty.

Fig. 7-22 October profit vs. average absolute deviation from schedule,

$\$ 5 / \mathrm{MWh}$ deviation penalty.

Fig. 7-23 Monthly profit for different quantile forecasts compared to bidding based on point forecast and based on the expected profit criterion, no penalty (left), \$5/MWh deviation penalty (right).

Fig. 7-24 Average hourly profit and CVaR for different quantile forecasts, no penalty (left), $\$ 5 / \mathrm{MWh}$ deviation penalty (right).

Fig. 7-25 Evaluation of probabilistic forecasts for different methods: calibration (left) and sharpness (right). Training period: January-July, 2006;

test period: August-December 2006. 105

\section{LIST OF TABLES}

Table 1-1 Different types of uncertainty representation in WPF. 3

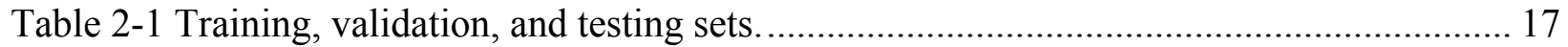

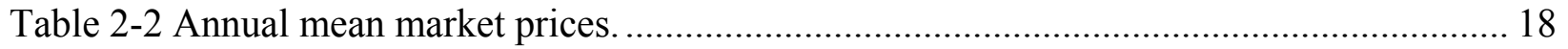

Table 2-3 Simulation of wind farm A's participation in the Iberian market. ............................ 19

Table 2-4 Simulation of wind farm B's participation in the Iberian market. ........................... 19

Table 2-5 Simulation of wind farm C's participation in the Iberian market. ............................. 20

Table 3-1 Overview of WPF and market operation in five U.S. electricity markets.

Based on information from the Utility Wind Integration Group (UWIG)

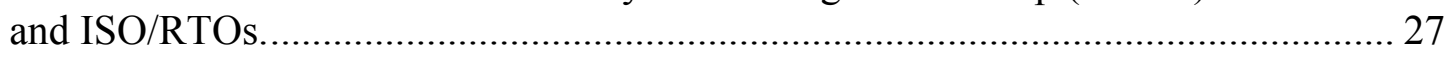

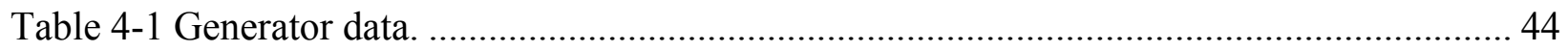

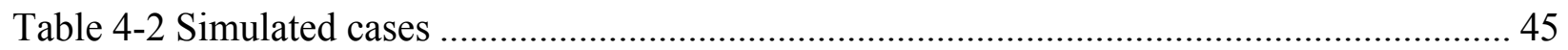

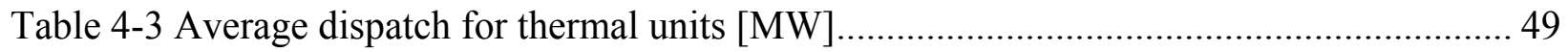




\section{LIST OF TABLES (CONT.)}

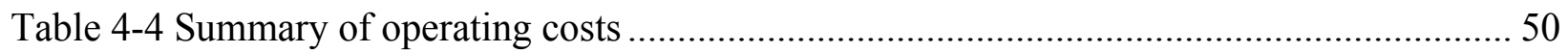

Table 4-5 Summary of other results.......................................................................... 50

Table 6-1 Summary statistics for scenarios (QR) with different reduction methods: average (AVG), mean absolute error (MAE), Oct.-Dec........................................ 74

Table 6-2 Simulated cases with deterministic UC ............................................................ 75

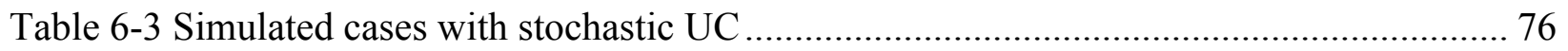

Table 6-4 Summary of other results: deterministic cases .................................................... 77

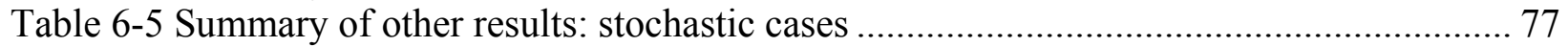

Table 7-1 Overview of published research/models for wind power trading. ........................... 84

Table 7-2 Price parameters and wind power point forecast (pf) for hour 5, Oct. 9, 2006.......... 94

Table 7-3 Summary of results at optimum for different decision criteria

(hour 5, Oct. 9, 2006), no deviation penalty......................................................... 97

Table 7-4 Summary of results at optimum for different decision criteria (hour 5, Oct. 9, 2006), \$5/MWh deviation penalty.................................................... 97

Table 7-5 Summary statistics for wind power and DA/RT prices, October............................ 100

Table 7-6 Summary of results for October with different decision criteria, no deviation penalty.

Table 7-7 Results summary for October with different decision criteria, $\$ 5 / \mathrm{MWh}$ deviation penalty.

Table 7-8 Summary of results for October with the $\mathrm{C}^{*}(\mathrm{w}=0.1)$ strategy for different wind power uncertainty forecasts, $\$ 5 / \mathrm{MWh}$ deviation penalty. 106 


\section{ACKNOWLEDGMENTS}

This report has been prepared by Argonne National Laboratory in collaboration with INESC Porto, Portugal. The authors acknowledge the U.S. Department of Energy, Office of Energy Efficiency and Renewable Energy through its Wind \& Water Power Program for funding the research presented in this report under contract DE-AC02-06CH11357.

Argonne National Laboratory, September 30, 2011. 


\section{ACRONYMS}

AGC automatic generation control

CAISO California Independent System Operator

CTE conditional tail expectation

CVAR conditional value at risk

DA

day-ahead

DOE

U.S. Department of Energy

ED economic dispatch

ERCOT Electric Reliability Council of Texas

EWITS Eastern Wind Integration and Transmission Study

GHG greenhouse gas

GW gigawatt(s)

ISO independent system operator

ITL information theoretic learning

KDE kernel density estimation

KDF kernel density forecast

LMP locational marginal prices

MAE mean absolute error

MCC Maximum Correntropy Criterion

MEE Minimum Error Entropy

MIBEL name of the Iberian electricity market

MISO Midwest Independent System Operator

MLP multilayer perceptron

MPC Minimum Penalty Costs

MPCC Maximum Parametric Correntropy Criterion

MSE Minimum Square Error

MW megawatt

NERC North American Electricity Reliability Corporation

NW Nadaraya-Watson

NWP numerical weather prediction

NYISO New York Independent System Operator

pdf probability density function

pmf probability mass function

PPA power purchasing agreement 


$\begin{array}{ll}\text { QC } & \text { quantile-copula } \\ \text { QR } & \text { quantile regression } \\ \text { RAC } & \begin{array}{l}\text { reliability assessment commitment } \\ \text { real time } \\ \text { RT }\end{array} \\ \text { regional transmission organization } \\ \text { SCADA } & \begin{array}{l}\text { supervisory control and data acquisition } \\ \text { security-constrained economic dispatch }\end{array} \\ \text { SCED } & \text { security constrained unit commitment } \\ \text { SCUC } & \text { system operator } \\ \text { SO } & \text { standard deviation } \\ \text { Std } & \text { unit commitment } \\ \text { UC } & \text { wind-to-power } \\ \text { W2P } & \text { wind generation company } \\ \text { WGENCO } & \text { wind power forecasting } \\ \text { WPF } & \end{array}$


This page intentionally blank. 


\section{SUMMARY}

The rapid expansion of wind power gives rise to a number of challenges for power system operators and electricity market participants. The key operational challenge is to efficiently handle the uncertainty and variability of wind power when balancing supply and demand in ths system. In this report, we analyze how wind power forecasting can serve as an efficient tool toward this end. We discuss the current status of wind power forecasting in U.S. electricity markets and develop several methodologies and modeling tools for the use of wind power forecasting in operational decisions, from the perspectives of the system operator as well as the wind power producer. In particular, we focus on the use of probabilistic forecasts in operational decisions. The main findings from each of the chapters are summarized below.

Statistical algorithms based on computational learning are typically used to convert weather forecasts and observational data to wind power point forecasts. In Chapter 2, we test different training criteria for a neural network applied to wind power point forecasting. We argue that the best choice of training criterion depends on the forecast user and the intended application of the forecast. In a case study with data from the Iberian electricity market, we illustrate the conflicting objectives between wind power producers and system operators, and how this conflict may influence the choice of training criteria for wind power forecasting. In the Iberian market, there is an assymmetry in the imbalance prices, and this gives the wind power producers an incentive to base their market bids on a biased forecast. However, such a strategy would give rise to larger deviations between scheduled and delivered energy, which is not in the interest of the system operator. By testing the traditional minimum square error criterion against several entropy-based criteria from information theoretic learning, we find that the maximum parametric correntropy criterion in this case gives results that are a compromise between the objectives of the system operator and wind power producer. We conclude that it is important for system operators to have their own unbiased forecast for reliability purposes.

We analyze the current status of wind power forecasting in U.S. electricity markets in Chapter 3. Most independent system operators (ISOs)/regional transmission operators (RTOs) in the United States have relatively limited experience with wind power forecasting. Still, wind power forecasting is already used for several important applications with a focus on maintaining reliability. As the wind power capacity is rapidly increasing, we find that there is a need to better integrate wind power forecasting into different parts of electricity market operations. Wind power forecasting can play an important role in the determination of operating reserve requirements, day-ahead scheduling and market clearing, reliability commitments, and real-time dispatch. At the same time, from a regulatory perspective it is also important to design electricity markets that give market participants the opportunity and right incentives to provide their unbiased forecast information through their scheduling and bidding decision. ISO/RTOs and market participants will need to develop new tools and procedures to make efficient use of the forecast information with the overall goal to make better operational decisions under the increased uncertainty and variability from wind power and other sources of renewable generation. A key challenge is therefore to improve decision making under uncertainty and the understanding of the impact of uncertainty on operational decisions. At the same time, it is important to continue the improvements of wind power forecasting models and to better tune 
them to the specific needs of the forecast users. From the perspective of system/market operators, it is particularly important to improve predictions of forecast uncertainty and ramping events.

We investigate the representation of wind power forecasting uncertainty in the unit commitment problem in Chapter 4. Traditional deterministic unit commitment models use a point forecast for wind power output. In contrast, we propose a stochastic alternative that captures forecast uncertainty by using scenarios that capture cross-temporal dependencies in the predicted wind power. We conduct a comparison of a diversity of unit commitment strategies on a test power system with ten thermal units and $20 \%$ wind power penetration. The results indicate that representing wind power forecasting uncertainty with wind power scenarios combined with stochastic unit commitment has advantages over the traditional deterministic approaches. Moreover, the stochastic model provides a rational and adaptive way to provide adequate spinning reserves at every hour, as opposed to increasing reserves to predefined, fixed margins that cannot account for the system's costs or assumed risks. However, the results also indicate that the scenarios do not capture the full range of wind power forecast uncertainty, and that it is prudent to impose a certain level of additional reserves also with stochastic unit commitment.

A set of scenarios is a convenient way to represent wind power uncertainty in decision problems with inter-temporal constraints. However, an extremely large set of elementary scenarios is needed to obtain an accurate representation of the probability density function covering the complete forecasting space. The size of this set is usually incompatible with complex algorithms such as the stochastic unit commitment problem, which is time demanding and requires high computational effort. In Chapter 5, we propose a methodology that can substitute a large scenario set by a smaller set of clusters. Each cluster is replaced by a representative scenario associated with the probability of the scenarios it represents. The reduction capability depends on the admitted tolerance, the distance metric used to define the clusters, and the dispersion of the original scenarios. The choice of the metric and the admissible tolerance should be defined in accordance with the decision problem, and the level of accuracy needed for the results. The method not only produces a set of focal or representative scenarios, but also orders them by probability value. This approach allows retaining a selected number of scenarios and understanding the risk of missing an adequate representation due to truncating the original scenario set. Furthermore, the necessary number of scenarios to satisfy a maximum level of risk can be chosen. This work, therefore, is a contribution to facilitating the adoption of stochastic optimization models in operational planning, for instance, to use as input to the stochastic unit commitment algorithms such as the one presented in Chapter 4.

In Chapter 6, we investigate in more detail the potential use of probabilistic wind power forecasting in the commitment of energy and operating reserves. Such forecasts can provide scenarios as input to a stochastic unit commitment or, alternatively, can be used to derive dynamic operating reserve requirements for use in deterministic unit commitment. One advantage of dynamic operating reserves is that this approach is better aligned with current operating procedures. Furthermore, the deterministic unit commitment model has a much lower computational burden. However, dynamic reserves do not capture the effect of inter-temporal ramping events. Moreover, this approach does not consider uncertainty and its cost in the objective function. In contrast, stochastic unit commitment does address inter-temporal variability through the scenario representation of uncertainty. Furthermore, the total cost, including the expected cost of scarcity, is explicitly taken into account in the objective function. However, the switch to stochastic unit commitment involves a more radical departure from 
current practice, it may run into computational constraints in large systems, and the benefits in terms of cost savings may be limited. In fact, our numerical results from the 10-unit test case, and also from a more realistic case study of the power system in Illinois, show that dynamic operating reserves and stochastic unit commitment give similar results in terms of cost and reliability although there may be distinct differences in commitment and dispatch decisions. Furthermore, we find that scenario reduction algorithms, such as the one presented in Chapter 5, tend to lower the variance in the reduced scenario set. In turn, this result reduces the amount of hedging built into the stochastic unit commitment solutions giving rise to a need for additional reserves. Finally, when comparing the use of different probabilistic forecasts, we find that forecasts based on kernel density estimation tend to give better operational results than forecasts from quantile regression, possibly due to better forecast calibration.

Future work is likely to improve the quality of probabilistic wind power forecasts, both in terms of the estimation of probability distributions and in the generation of adequate scenarios. However, there will always be inaccuracy in probabilistic forecasts, just as in point forecasts. This fact calls into question the overall risk paradigm under which the system operator's scheduling decisions are made. In stochastic unit commitment, where the objective is to minimize the expected cost, one can adjust the risk level by changing the costs of unserved energy and reserves. However, other approaches based on different decision paradigms — such as utility theory, value at risk, robust optimization, or regret - should also be considered. Ultimately, the optimal decision strategy does not only depend on the risk preferences of the system operator, but also on the quality of the probabilistic forecast.

Finally, in Chapter 7 we investigate the potential use of probabilistic forecasting for wind power producers. We describe a model for optimal trading of wind power in day-ahead electricity markets under uncertainty in wind power and prices. The model considers settlement mechanisms in markets with locational marginal prices, where wind power is not necessarily penalized from deviations between day-ahead schedule and real-time dispatch. We use utility theory and conditional value at risk to represent the risk preferences of the wind power producer. The model is tested on data from a hypothetical wind farm located in the state of Illinois combined with historical price data. The results demonstrate that probabilistic wind power forecasts can play an important role in guiding wind power trading decisions in the day-ahead market. The results also indicate that price forecasts are important in order to derive good trading strategies, particularly under market designs without penalties for deviations between day-ahead schedule and real-time delivery. Furthermore, risk preferences clearly influence the optimal trading decisions, with risk averse owners bidding less energy into the day-ahead market to reduce their exposure to high real-time prices. The decisions are also highly dependent on market design, and the results show that a deviation penalty drives the optimal day-ahead bid closer to the point forecast for all strategies. This is in the interest of the system operator, because it makes the task of balancing supply and demand in the system an easier one. However, deviation penalties also add a significant financial burden on wind power producers and therefore reduce the profitability of wind energy. Revising market rules to give efficient operational incentives without adding an undue burden on wind power and other renewables will clearly be a challenge as we move forward. Overall, we conclude that stochastic models will be important tools to control the trade-off between risk and return for wind power producers participating in electricity markets. Improved scheduling decisions and better risk management will increase the viability of 
wind power in the long run, and our analysis shows that wind power forecasting can serve as an important tool to achieve this goal. 


\section{INTRODUCTION}

Driven by increasing prices for fossil fuels and concerns about greenhouse gas (GHG) emissions, wind power, as a renewable and clean source of energy, is rapidly being introduced into the existing electricity supply portfolio in many parts of the world. The U.S. Department of Energy (DOE) has analyzed a scenario in which wind power meets $20 \%$ of the U.S. electricity demand by 2030, which means that the U.S. wind power capacity would have to reach more than 300 gigawatts $(\mathrm{GW})$ [1]. The European Union is pursuing a target of 20/20/20, which aims to reduce greenhouse gas (GHG) emissions by $20 \%$, increase the amount of renewable energy to $20 \%$ of the energy supply, and improve energy efficiency by $20 \%$ by 2020 as compared to 1990 [2]. Meanwhile, China is the leading country in terms of installed wind capacity, and had $45 \mathrm{GW}$ of installed wind power capacity out of about $200 \mathrm{GW}$ on a global level at the end of 2010 [3].

The rapid increase in the penetration of wind power into power systems introduces more variability and uncertainty in the electricity generation portfolio, and these factors are the key challenges when it comes to integrating wind power into the electric power grid. Wind power forecasting (WPF) is an important tool to help efficiently address this challenge, and significant efforts have been invested in developing more accurate wind power forecasts. In this report, we document our work on the use of wind power forecasting in operational decisions.

\subsection{Wind Power Forecasting}

A WPF system, as illustrated in Fig. 1-1, uses input data from different sources, including results from numerical weather prediction (NWP) models, local meteorological measurements, supervisory control and data acquisition (SCADA) data describing the real-time (RT) state of the wind power plants, and additional information about the characteristics of the wind power plants and the nearby terrain and topography. WPF models are typically based on a combination of physical and statistical models. The physical approach describes the physical relationship between atmospheric conditions, local topography, wind speed, and the output from the wind power plant. In contrast, the statistical approach estimates a statistical relationship between all the relevant input data and the resulting wind power generation without considering the physics of the system. The performance of wind power forecasts and the forecast accuracy depends on the availability of good NWP forecasts, the complexity of the terrain, and the availability of RT weather and wind power plant data. Hence, there can be large differences in forecasting errors between wind power plants at different locations. WPF systems typically produce forecasts for a time horizon up to 2-3 days ahead in time. In general, the forecasting error increases with the forecast horizon.

There are two common forms of delivering the wind power forecast: point forecast and uncertainty forecast. Through the course of this project, we have developed and tested improved statistical algorithms for both types of forecasts, as briefly outlined below. We provide an extensive overview of the state-of-the-art in WPF in [4]. A complete documentation of our work on improved statistical WPF algorithms is available in [5]. 

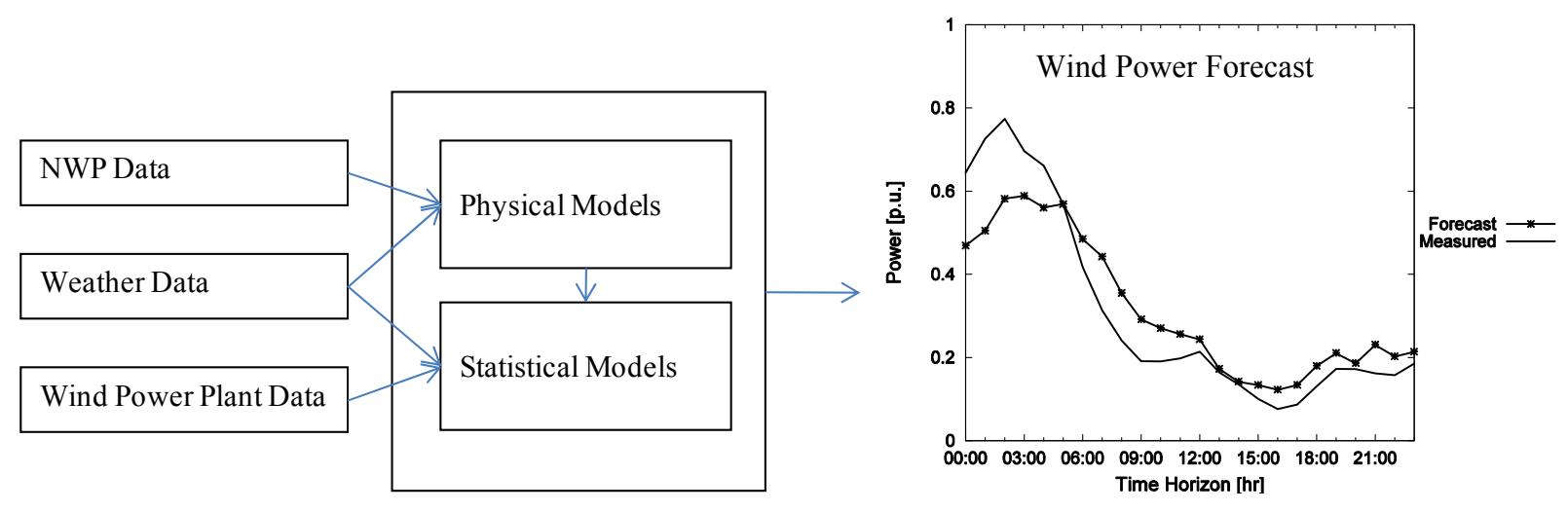

Fig. 1-1 Illustration of WPF system.

\subsubsection{Wind Power Point Forecasting}

A point forecast represents a single value for each look-ahead time horizon, which contains the conditional expectation at each time step. The point forecast estimation is typically based on statistical training criteria (e.g. by minimizing the square error [MSE]). We have analyzed the impacts of adopting different statistical training criteria for point forecasting models, focusing on the comparison of different forecasting error metrics. Our study, documented in [5], showed a significant degree of dependence on the training criterion applied, also pointing out the importance of online training. Specifically, the application of information theoretic learning (ITL) training criteria in wind power point forecasting, using neural networks, was tested for two wind farms located in the U.S. Midwest. The advantage of using such metrics over the MSE criterion was confirmed in these experiments. However, a point/deterministic forecast cannot provide information about the dispersion of observations around a predicted value. Moreover, an expectation cannot help decision makers in calculating risks or the conditional values at risk. Therefore, it is essential to generate, together with (or as an alternative to) point forecasts, a representation of the wind power uncertainty by a description of the probability density function (pdf).

\subsubsection{Wind Power Uncertainty Forecasting}

Uncertainty forecasts estimate the marginal distributions for each time step, for example, in the form of intervals, quantiles, or scenarios, which represent information about the uncertainty. Such forecasts can help forecast users in their decision-making processes (e.g., enabling derivation of advanced strategies for market participation). Wind power uncertainty comprises three key features: model chain, uncertainty representation, and time-adaptive models. A traditional model chain consists of using the forecast errors of point forecasts from a deterministic WPF model as input to the uncertainty estimation. The wind power uncertainty representation is determined by the end-user's requests and the nature of the decision-making problem. It can take the form of probabilistic forecasts, risk indices, or scenarios for short-term wind power generation (Table 1-1). In general, one can only talk about more or less adequate uncertainty representations, rather than better or worse ones. However, the pdf representation is the most complete and flexible, enabling a range of different decision-making problems in the power system domain. By using a time-adaptive model, the forecasting system is able to cope with non-stationary data and, therefore, to adapt to the new situation without requiring additional offline training of the model. 
Table 1-1 Different types of uncertainty representation in WPF.

\begin{tabular}{|c|c|}
\hline \multicolumn{2}{|c|}{ Uncertainty Representation } \\
\hline \multirow{4}{*}{ Probabilistic } & Quantiles \\
\hline & Interval Forecasts \\
\hline & Probability Mass Function \\
\hline & Probability Density Function \\
\hline \multirow{2}{*}{ Risk Indices } & Meteo Risk Index \\
\hline & Prediction Risk Index \\
\hline \multirow[b]{2}{*}{ Scenarios of Generation } & Scenarios with Temporal Dependency \\
\hline & $\begin{array}{c}\text { Scenarios with Spatial/Temporal } \\
\text { Dependency }\end{array}$ \\
\hline
\end{tabular}

Our uncertainty forecasting studies, documented in [5], tested two new kernel density forecast (KDF) methods applied to the wind power uncertainty problem in both offline and time-adaptive modes: the Nadaraya-Watson (NW) estimator and quantile-copula (QC) estimator. Both the Eastern Wind Integration and Transmission Study datasets, as well as two Midwest wind farms, were used in different case studies and were compared to linear and splines quantile regression (QR) methods, which is the current state of the art. The results show that the KDF and QR methods have similar performance in terms of sharpness, resolution, and skill score, although the former lead to a better calibration. In addition, we found that time-adaptive approaches for both NW and QC change the bias of the probabilistic forecasts (i.e., calibration), while only slightly changing the sharpness and resolution. Moreover, the time-adaptive mode improves the skill score when compared to the offline approach.

An alternative way of presenting the wind power uncertainty is by using a discrete set of scenarios, sampled according to a probability density function associated with the forecasts, and therefore able to characterize the uncertainty according to the historical error distribution. Another advantage of a scenario set is that it can include the temporal dependency information of the errors. However, in order to capture the entire characteristic of the wind power forecasted during the prediction period accurately, a decision maker may need to evaluate a high number of scenarios. This is a time-consuming and computationally demanding task — and the computational burden usually is not compatible with stochastic programming algorithms run in useful time. Therefore, there is typically a need to select only a small number of scenarios that can represent the whole scenario set at best. We explore scenario reduction algorithms and their application in the power system domain in more detail in this report.

\subsection{Wind Power Forecasting in Operational Decisions}

Different agents in the electricity market may have conflicting interests as they interact in the market environment. Wind power producers typically aim at minimizing their forecasting error over a longer period of time, in order to better schedule their generation. However, their main concern is in maximizing income. System operators, on the other hand, are concerned with guaranteeing the security of the system. System operators are interested in minimizing operational costs while maintaining a high level of reliability. This implies that the choice of a WPF model is not neutral and, in some cases, the definition of what constitutes a good wind power forecast might differ between forecast users. For a long time, a "good" forecast has been considered to fit predictions closest to the observed values, in what is known as the forecaster's 
paradigm. This idea has given place to the forecast consumer's paradigm, which states that forecasts should be tuned toward the user's interests. With this (new) relative concept, we can assess the forecast either in terms of consistency (correspondence between forecasters' judgment), quality (correspondence between forecasts and observations), or value (incremental benefits due to implementation into decision-making process). We focus on system operators and wind power producers as two distinct and different WPF users in this report.

\subsubsection{System Operators}

From the perspective of a system operator, its concern in guaranteeing the security of the system is closely related to WPF uncertainty. In fact, large unexpected changes in wind power output over a short period of time is one of the major issues in power system operations with wind power. In the presence of such ramping events, system operators have to develop operational procedures in order to satisfy the load and maximize both the economical and environmental benefits. The longer the time ahead of such events that they can be predicted, the more options a system operator has to balance the system. However, forecast uncertainty generally increases with the forecast horizon. A key challenge for the system operator is therefore to handle the uncertainty and variability from wind power in a cost-efficient manner without compromising system reliability. System operators will need to adopt new methods to better handle wind power and other renewable resources in their scheduling and dispatch decisions. In this study, we tackle this problem from two ways. One way is to extend the traditional operating reserve strategies, that is, to schedule appropriate amounts of reserve capacity based on information from wind power uncertainty forecasting. Another way is to rethink the formulations of the power system scheduling and market clearing problems. We explore a stochastic formulation of the unit commitment (UC) problem and analyze to what extent stochastic programming approaches can deal with the wind power uncertainty.

\subsubsection{Wind Power Producers}

From the perspective of a wind power producer, it is becoming increasingly important to manage the risk of participating in the electricity market. A majority of the wind power in the United States is sold under long-term power purchasing agreements (PPAs), which lock in the price of delivered electricity and thereby stabilize the revenue stream. However, a significant share of wind power is being sold directly to the electricity market. It is obviously important for merchant wind generation to apply scheduling and trading strategies that reflect the uncertainty in wind power and prices and the corresponding trade-off between risk and return. Buyers of PPAs also have to schedule the contracted wind power into the electricity market and must consider how wind power influences the scheduling strategy of the rest of their portfolio. Trading of wind power in electricity markets is still a relatively new problem. In this report, we investigate the scheduling and trading of wind power focusing on electricity markets in the United States with locational marginal prices (LMPs), including settlement procedures and their influence on trading decisions. Moreover, we develop an optimization model for wind power trading under uncertainty. The model can find the optimal wind power bid under uncertainty in forecasts for wind power and prices (day-ahead and real-time, considering the risk preferences of the wind power producer and the impact of potential deviation penalties. 


\subsection{Report Outline}

This report documents our research on the application of wind power forecasting for both system operators and wind power producers. The report is organized as follows: Chapter 2 reports a study of WPF training criteria and the "goodness" of a forecast, focusing on the electricity market in Spain and Portugal; in Chapter 3, we discuss the current use of WPF in U.S. independent system operator (ISO)/regional transmission operator (RTO) markets; Chapter 4 presents our implementation of WPF uncertainty in a stochastic formulation of the UC problem, as well as a comparison among different UC strategies; Chapter 5 presents a new clustering-based scenario reduction method for generation of adequate WPF scenarios for use in stochastic programming models; in Chapter 5, we present a comparison between different probabilistic forecasting and scenario reduction methods and test the resulting forecasts on the stochastic UC model from Chapter 4; Chapter 7 describes a new model for optimal trading of wind power in day-ahead electricity markets under uncertainty in wind power and prices, considering settlement mechanisms in the electricity markets with LMPs; finally, Chapter 8 summarizes the overall conclusions from our work on the use of WPF in operational decisions.

Note that for many of the chapters, the content has been published in conference proceedings or journal papers. References to underlying publications are provided in footnotes at the beginning of each chapter. 
This page intentionally blank. 


\section{GOOD OR BAD WIND POWER FORECASTING: A RELATIVE CONCEPT ${ }^{1}$}

This chapter reports a study on the importance of the training criteria for WPF and calls into question the generally assumed neutrality of the "goodness" of particular forecasts. The study, focused on the Spanish electricity market as a representative example, combines different training criteria and different users of the forecasts to compare them in terms of the benefits obtained. In addition to more classical criteria, an Information Theoretic Learning (ITL) training criterion, called parametric correntropy, is introduced as a means to correct problems detected in other criteria and to achieve more satisfactory compromises among conflicting criteria, namely forecasting value and quality. We show that the interests of wind farm owners may lead to a preference for biased forecasts, which may be in conflict with the larger needs of secure operating policies. The ideas and conclusions are supported by results from three real wind farms.

\subsection{Introduction}

The demand for more accurate, short-term WPF models has led to solid and impressive development in recent years [4][6]. Increasing the value of wind generation by improving WPF systems' performance - and furthering its integration into operational management - is one of the priorities in current research [7]. On many past occasions, energy market participants have taken for granted that a "good" forecast would be something that turns out to be as close to the actual values as possible. This view is called the forecaster paradigm, where the concern is to fit the prediction to the observed values in the signal processing sense. A good example of a WPF evaluation protocol in agreement with this paradigm is [8]: the main concern is minimizing the forecasting error, and no importance is given to the nature and consequences of the errors. However, it may be argued that the definition of the goodness of a forecast should be strongly related to the use given to such a forecast. In the forecasting pilot project by the Alberta Electric System Operator [9], an important idea is that a forecast should be oriented by the user's interests. Forecasting errors may be seen through many perspectives (e.g., mean, bias, maximum deviation, phase error, etc.), and the nature of the errors may be too broad - one particular forecast is not likely to be considered optimal simultaneously in distinct applications, such as market participation or power system operation.

This situation has been recognized in the domain of weather forecast. Several publications discuss what a good or bad forecast is. Murphy [10] distinguished three different types of goodness in forecasts: (1) the correspondence between forecasts and forecasters' judgments (consistency); (2) correspondence between forecasts and observations (quality); and (3) the incremental benefits (economic/or other) when employed by users as an input into their decisionmaking processes (value). The factor of consistency in WPF is not discussed in this chapter: it is assumed to be included by the forecaster in the model during the development phase. In order to assess the quality of WPF, the standard approach is based on statistical error measures (e.g., mean absolute error) [8]. However, this approach does not guarantee that the forecast with the best score is the one with best quality. An alternative approach, called verification framework, was presented by Murphy and Winkler [11]. This approach is based on the joint distribution of forecasts and observations and factorization of this distribution into conditional

\footnotetext{
${ }^{1}$ This chapter is reproduced from Wind Energy, Vol. 14, No. 5, R.J. Bessa, V. Miranda, A. Botterud, J. Wang,

“'Good' or 'Bad' Wind Power Forecasts: A Relative Concept," pp. 625-636, 2011, with permission from Wiley.
} 
and marginal distributions. Pinson [12] used this framework, named the "distribution-oriented approach," and applied it to WPF. His objective was to study the main characteristics of WPF uncertainty.

The third type of forecast goodness, forecast value, is often very difficult to quantify, as in some cases it is related to non-economic factors. However, some decision makers may give a high relevance to these criteria [13]. Some researchers have reported incremental economic benefit from the use of WPF in the participation of wind power in electricity markets. Usaola et al. [14] quantified the economic advantages of using an advanced forecasting approach that takes as input numerical weather predictions (NWPs) instead of persistence. Barthelmie et al. [15] estimated the wind farm size for which WPF outweighs the cost of installing or implementing short-term forecasts. The authors conclude that the economic benefit of using WPF depends on the accuracy and cost of purchasing the forecast. Angarita-Márqueza et al. [16] estimated the income obtained by a wind farm in the Spanish and British markets by using an advanced forecasting system (Sipreólico) compared to persistence. In the British market, the Sipreólico system produced results similar to persistence because the period between gate closure and actual delivery was very short. For the Spanish market, because the period between gate closure and delivery was longer, Sipreólico improved the income amount(s) when compared to persistence.

Pinson et al. [17] studied the use of WPF probabilistic forecasts to support the participation of wind generation in the electricity market. The authors showed that with the information about uncertainty, it is possible to derive advanced strategies for market participation leading to a significant increase in income when compared to persistence and point forecasts obtained with fuzzy-neural networks. Fabbri et al. [18] proposed a probabilistic methodology for estimating the WPF error costs in the electricity market. Other related works can be found in [19]-[21].

One crucial aspect is that the relationship between quality and value is nonlinear and differs from problem to problem, as well as from user to user [22]. Hence, it is important to recognize that there are different users for WPF, the two most important being wind generation companies (WGENCOs) that bid their wind generation into the electricity market and system operators (SOs) that must guarantee the security of the system.

The output of a forecasting process or model can therefore be evaluated according to two paradigms, which we will call the forecaster paradigm and the forecast consumer paradigm. The former is mainly concerned with the time series in itself, in the signal processing sense, and is therefore related with quality; the latter focuses on the use given to the forecasts and is therefore related to value.

The main idea driving this chapter is to promote the recognition (many times forgotten) that distinct user groups may have conflicting interests in a restructured market environment, and therefore the choice of a model is not neutral. For instance, SOs are interested in minimizing operational costs while maintaining a high level of reliability. In contrast, WGENCOs are mainly interested in maximizing their income levels in the electricity market. Hence, in some conditions, the definition of a "good" forecast varies with different forecast users. 
This chapter contributes to the discussion of this topic by comparing the forecast errors and economic consequences of adopting several criteria for the statistical training of WPF models. To illustrate the relevance of the concepts discussed, the chapter presents a case study of the Iberian day-ahead (DA) electricity market (MIBEL). The study could be reproduced for other markets; however, the objective here is not to study and compare markets but to discuss models, and the example presented is sufficiently representative. Our main objective is not to provide an alternative to the approaches based on probabilistic forecasts [17]-[21] but rather to highlight a new view of the relative importance of the forecast error in a multi-criteria and multi-perspective paradigm, in which biased point forecasts could be produced to increase the market income, and to stress the conflicting objectives that may exist between different users (or stakeholders). To compensate for some of the problems identified and analyzed, we introduce parametric correntropy, which is a new training criterion for WPF that aims to arrive at a satisfactory compromise between forecast value and quality.

We first discuss the WGENCOs' and SOs' perspectives on WPF. Next, on the basis of actual DA market data from MIBEL, we illustrate the conflict between the viewpoints and also the applications of different training criteria. Finally, we analyze the consequences of the different training criteria for the two WPF users.

\subsection{The "Forecast Consumer Paradigm"}

\subsubsection{Wind Generation Companies' Viewpoint}

In a restructured market environment, forecast errors may have an associated cost or penalty, as the wind power producer usually ends up supplying an amount of energy that differs from the market bid. The market remuneration scheme adopted in this chapter is for operating in the DA market and is analogous to the one formulated in [17]-[21].

In this mechanism, wind power producers offer energy quantity bids $\left(E^{b}\right)$ at day $D$ (typically till 12:00 noon) for every hour of day $D+1$ in the DA market, and they are paid at the market clearing price $\left(p^{s}\right)$. Then, if the actual wind generation is above or below their bid, they will be subject to a penalty as follows:

- If the actual generation $\left(E^{p}\right)$ is above the market bid, the excess is paid at a discounted price $\left(p^{\text {surplus }}\right)$. This price can also be negative, which represents a cost.

- If the actual generation remains below the market bid, a penalty is applied according to the price $\left(p^{\text {shortage }}\right)$ of the generation that the SO has to purchase to compensate for the insufficient generation.

Each market specifies its own rules for the calculation of deviation costs, and therefore, no general conclusion can be offered. However, it seems obvious - and this chapter confirms this finding — that if the penalties are asymmetric, then an opportunity arises for a gambler to benefit from using a biased model instead of a neutral model.

A wind farm's income $(I)$ from selling for a given look-ahead time $t+k$ can be formulated as: 


$$
I_{t+k}=\left\{\begin{array}{l}
p_{t+k}^{s} \cdot E_{t+k}^{b}+p_{t+k}^{\text {surplus }} \cdot\left(E_{t+k}^{p}-E_{t+k}^{b}\right), E_{t+k}^{p}>E_{t+k}^{b} \\
p_{t+k}^{s} \cdot E_{t+k}^{b}-p_{t+k}^{\text {shortage }} \cdot\left(E_{t+k}^{b}-E_{t+k}^{p}\right), E_{t+k}^{p}<E_{t+k}^{b}
\end{array}\right.
$$

This formulation can be rearranged by considering the balancing prices, $c_{\text {down }}$ reg $=p_{s}-p_{\text {surplus }}$ and $c_{\text {up_reg }}=p_{\text {shortage }}-p_{s}$, as:

$$
I_{t+k}=\left\{\begin{array}{c}
p_{t+k}^{s} \cdot E_{t+k}^{p}-c_{t+k}^{\text {down_reg }} \cdot\left(E_{t+k}^{p}-E_{t+k}^{b}\right), E_{t+k}^{p}>E_{t+k}^{b} \\
p_{t+k}^{s} \cdot E_{t+k}^{p}-c_{t+k}^{u p_{-} r e g} \cdot\left(E_{t+k}^{b}-E_{t+k}^{p}\right), E_{t+k}^{p}<E_{t+k}^{b}
\end{array}\right.
$$

It is important to emphasize that in this market formulation, the WGENCOs are appointed balance responsibility; hence, they have a financial responsibility for any imbalance.

In some markets, if the deviation helps the SO balancing measure, then the market agent is not penalized. Note that the intra-day markets are not taken into account but may reduce the forecast error - and consequently the deviations - considerably.

From the WGENCO viewpoint, the value of the WPF in the market is translated by the additional income provided by such forecasts over the income obtained with an alternative method. The first component of (2-1) is constant and corresponds to the income obtained with perfect forecasts. Hence, maximizing the market income is analogous to minimizing the expected costs of balancing (second component in [(2-2)]). Therefore, in order to meet its goal, the WGENCO will follow a particular bidding strategy depending on the market balancing costs.

Because of the uncertainty in prices and wind generation, resolving this matter is a decision problem under uncertainty. Several decision rules can be used for finding the preferred bid. The decision rule used most often is to find the bid $E_{t+k}^{b}$ that minimizes the expected balancing costs (i.e., to maximize expected income). This rule was first introduced by Bremnes [23] and has been used by authors in [17] and [21]. This decision rule describes a risk-neutral attitude (i.e., a linear utility function). Bourry et al. [20] presented an alternative strategy based on portfolio theory that balances between the expected value and the risk of the balancing costs distribution. Botterud et al. [24] present a methodology to derive optimal DA bids for a wind power producer under uncertainty and compare the results of different bidding strategies (e.g., maximizing the utility). The authors discussed how the optimal bids depend on the electricity market design.

Under the market assumptions outlined above, the "optimal" bid of the expected value decision rule possesses an analytical solution; for a proof see [23]. The bid that maximizes the expected income is the $c_{\text {down_reg }}\left(c_{\text {down_reg }}+c_{\text {up r reg }}\right)^{-1}$ quantile of the forecasted probability distribution of wind generation. It is found that the "optimal" bid depends only on the quantile proportion computed from the balancing prices. For instance, if $c_{\text {down_reg }}>c_{\text {up_reg, }}$, the "optimal" quantile is above 0.5 (median), and this result constitutes overestimation; conversely, if $\mathrm{c}_{\mathrm{down} \_ \text {reg }}<\mathrm{c}_{\text {up_reg, }}$, the "optimal" quantile is below the median and the result is underestimation.

Based on this idea and as carried out by Pinson et al. [17], the forecast with higher economic value is not necessarily the one with lower forecast error (in accordance with the "forecaster's paradigm"). The results presented by the authors support this idea: a point forecast with $40.55 \%$ 
of imbalances (with respect to produced energy) achieved an income of $1.15 \mathrm{M} €$ over one year, whereas an advanced strategy based on a probabilistic forecast reached a higher $55.46 \%$ of imbalances — but also an income of 1.21 M€.

As mentioned before, if the deviation is in favor of the system, then the WGENCO does not pay balancing costs under some markets' rules. In [21], the bidding strategy proposed by Bremnes [23] was extended to include this possibility, assuming that the system balance is unrelated to the wind power imbalance or that all wind power producers follow the same bidding strategy.

Finding the decision rule and the best bidding strategy is clearly a complex problem that requires wind power and price forecasting information. One alternative is to develop economic-oriented training criterion for training wind-to-power (W2P) models. Ravn [25] analyzed a training criterion that penalizes according to the balancing costs and the sign of the forecast errors:

$$
\min \sum_{J^{d o w n}} w_{\text {down_reg }} \cdot\left|\hat{P}_{t+k}-P_{t+k}\right|+\sum_{J^{u p}} w_{\text {up_ } r e g} \cdot\left|\hat{P}_{t+k}-P_{t+k}\right|
$$

The expression is divided into terms of overestimation, $J^{u p}\left(\hat{P}_{t+k}>P_{t+k}\right)$, and underestimation, $J^{\text {down }}\left(\hat{P}_{t+k}<P_{t+k}\right) ; w_{\text {down_reg }}$ and $w_{\text {up_reg }}$ are penalization factors related to the market balancing costs. By applying this training criterion, an economic bias is introduced, which can increase the forecast error, but also increases the income from the market. Therefore, the economic value of a wind power forecast can be increased by information provided by either probabilistic forecasts or economic-oriented deterministic (point) forecasts.

\subsubsection{System Operator's Viewpoint}

In our discussion, the viewpoint of the power SO is not linked to how the SO should make the forecasts. In Botterud et al. [26], the current use of WPF in U.S. ISO/RTO markets is discussed, and recommendations for how to make efficient use of the information in state-of-the-art forecasts are offered.

In this section, the discussion is related instead to the impact on the operational policy from the economic-oriented market bids provided by the WGENCOs. As stated in the previous section, a WPF system that provides the maximization of income to a WGENCO should not be trusted or used by SOs.

The SO needs to manage the combination of generation and consumption variability considering unit outages. Therefore, in a restructured electricity market environment, the SO acquires all of the reserve needed for the control area, in order to maintain a minimum reliability level.

In the daily market, the SO at day $D$ is in charge of defining and contracting the operating reserve (i.e., the reserve related to loss of load or generation surplus attributable to forecast errors — both in load and wind - and unit outages (e.g., spinning and non-spinning reserve) needed for the next day (day $D+1$ ) [27]. These operating reserve levels are generally settled by deterministic criteria, such as the capacity of the largest unit plus an empirical formula, such as the Union for the Coordination of Transmission of Electricity (UCTE, now ENTSO-E) rule [28]. 
However, in the literature, probabilistic approaches are proposed by several authors; see, for example, [29] and [30].

The integration of large shares of wind generation requires an increase in the amount of operating reserve that is needed to balance generation and load in order to maintain an acceptable level of reliability. Results from [29] and [31] support this idea. Despite reducing wind power variability as a result of the aggregation of several wind farms, the variability and uncertainty of wind generation tend to be a source of stress for the operations personnel.

Because the load and generation are dispatched by the market mechanism, and assuming that WGENCOs participate with bids in the market, the strategic bidding followed by WGENCOs may influence significantly the needs for operating reserves and also the overall cost of balancing the system. As mentioned in the previous section, a WGENCO's income does not necessarily increase by reducing forecast deviations. Therefore, the forecast error should be considered to be of secondary concern for the $\mathrm{SO}$, because the relevant error in this case is introduced by the market bids.

For instance, if the market bids overestimate the wind generation, then the load will be met by "untrue" generation in the DA market. In this case, the SO with its own neutral WPF system can forecast the wind generation uncertainty and by using, for instance, an approach that is similar to that used in [30] to detect a high risk of not meeting the load. Hence, the SO will need to commit more generating units (e.g., using the reliability assessment commitment to commit more units [26]) or to contract more upward reserve, which increases the operational costs.

The need for upward reserves is the most critical situation, even for planning the power system capacity, as it requires that traditional power station capacities must be available as operating reserves. This situation may limit the ability of wind power plants to replace the conventional power station capacities.

Of course, this system balancing cost depends on the generation mix (e.g., the reserve provided by gas turbines or hydro is less expensive) and on the operating strategy [32]. For instance, in cases where the SO defines the reserve requirements on the basis of deterministic criteria, the awareness of this overestimation may lead to a highly conservative attitude and an adoption of high safety margins to minimize the risk - and will therefore heavily increase the operational costs. In addition, as mentioned in [33], imbalances may aggravate potentially large out-of-merit automatic generation control (AGC) costs. All of these operational costs will be part of the tariff paid by all customers.

Nevertheless, the SO may have to shed load if it has offline units because of the expected wind power availability (as bid into the market). It may also happen that the deviation between load and generation cannot be met by all of the available operating reserve because of transmission constraints.

The same behavior is valid for the case where the wind power bids are underestimated. This problem is of less concern for the SO, because it does not need to shed load or have additional power stations available to cover the energy deficit. However, the SO also needs to contract 
downward reserve (e.g., reserve to deal with generation surplus due to forecast errors, e.g., pumped storage) and may have to curtail wind generation. This situation leads to what is called an "over-commitment" of conventional generation, which will reduce the economic and environmental welfare of the power system.

At this point, the key question from an SO viewpoint is: what is a "good" forecast (or bid) for the market made by WGENCOs? The "UCTE NetWork of Experts on Wind Power," composed of experts from several SOs such as Transpower (Germany) and REE (Spain), stated in [34] that the crucial issue is the expected maximum forecast deviation, and not the mean forecast error. The group's finding is because the extreme deviations may lead to load shedding. Consequently, for an $\mathrm{SO}$, a good criterion will be to minimize the expected maximum forecast error. However, this training criterion may be difficult to translate to a function, so the minimum mean square error (MSE) could be an acceptable criterion because it weights the large deviations more heavily.

However, this criterion must be viewed from the perspective of maintaining a desired reliability standard based on energy offered in the market by the market participants, including WGENCOs. In this case, a "good" WGENCO forecast for an SO should be the one that leads to: (1) less use of reserves, in particular upward reserves; (2) a lower expected maximum forecast error; and (3) deviations in favor of the system.

The first two criteria may lead to a conflict with the WGENCO's viewpoint. With respect to the third item, it is important to point out that if all WGENCOs use a bidding strategy based on the system deviation, it is probable that their deviations will be against the system deviation. Even in a situation where forecast errors help the system deviation, the SO must (in the day before) contract reserve and schedule the generation according to a probabilistic algorithm or deterministic rule without knowing this information a priori.

\subsubsection{The Compromise Viewpoint, or the Search for Utopia}

The forecasting and market bidding analysis must be performed carefully, because we are dealing with two generally conflicting viewpoints, as outlined above. In this case, there is no direct application of the trade-off concept, because there is no single agent that evaluates its gains in one criterion for giving up some value in another criterion - with disjoint or partially disjoint sets of criteria, a loss in one criterion is felt by an agent by just a loss. This analysis pertains to the field of multiple agent decision making, where it is recognized that the "Utopia" solution (i.e., one that optimizes all criteria for all agents, even those with conflicting interests and who may not even share the same criteria) is generally an infeasible point. Utopia corresponds to the highest level of aspiration for all agents. In contrast, the "ideal" solution refers to the maximum aspiration of a single agent and is therefore an individualistic concept.

Market participants therefore seek solutions that may be seen as an acceptable compromise by the several agents. These problems are solved either by negotiation or by arbitration (if one excludes the use of force); in the former case, the mode is unsupervised, and in the latter, it is a supervised mode. The relations between Utopia and the negotiation solutions have been extensively studied: the Nash bargaining solution (a Pareto-set solution that is the most equitable), the Kalai-Smorodinsky solution (providing more weighting to the "needier" player), and the Gupta-Liven solution (a Pareto-set solution in the line connecting the reference or focal point solution with Utopia) are just examples [35][36]. 
In our study, this function is not carried out through a negotiation process but by adopting the concept of a "compromise viewpoint" through the external assumption of what a "good" compromise should look like - this concept resembles the arbitration process, where an external independent agent with supervisory powers (e.g., a regulator) may decide on the characteristics of compromises among agents by considering criteria (or utility functions) pertaining to all agents in a joint framework.

It is natural to measure the forecast value relative to the income obtained by the market participant. However, the "optimal" forecast for a WGENCO may not be desired by the SO, because this agent considers, in its own evaluation of solutions, distinct criteria from those that the WGENCOs consider, namely, costs that are not included in the deviation costs of (2-2): for instance, cost of energy not supplied, contracted reserve capacity, and environmental costs. The aim in our approach is to offer a WPF training criterion function that suggests an acceptable compromise between the two viewpoints. In the following section, several training criteria described in the literature are presented.

It must be added, however, that an alternative exists: the decoupling of the problem - that is, when the distinct agents work with separate forecasts and do not take into account, for their own decisions, the forecasts used by the other parties. In that situation, each agent can adapt the models and training processes to accommodate its own interests without entering into direct conflict with the others.

\subsection{Training W2P Models}

\subsubsection{Aspects of Training W2P Models}

A W2P model is a term used to designate a neural network, a fuzzy inference system, or, in general, any system that emulates an input-output transfer function and whose performance depends on the tuning of internal weights or parameters, which are used to translate wind characteristics (e.g., wind speed, direction) into electric power. We can identify three basic modules in a W2P: its internal structure, the training criterion, and the optimization algorithm. Three types of actions can be applied in order to constrain the training of a W2P model (see Fig. 2-1) in:

- The internal structure, by modifying the number of weights;

- The training criterion, by selecting an adequate measure of performance related with the forecast error between the WP2 output $(y)$ and target value (T) (e.g., mean square error);

- The optimization algorithm, by choosing a mechanism or procedure (e.g., gradient descent) to close a feedback loop that updates the weights as a function of the training criterion computed for a training dataset.

This chapter addresses the aspect of training criterion. 


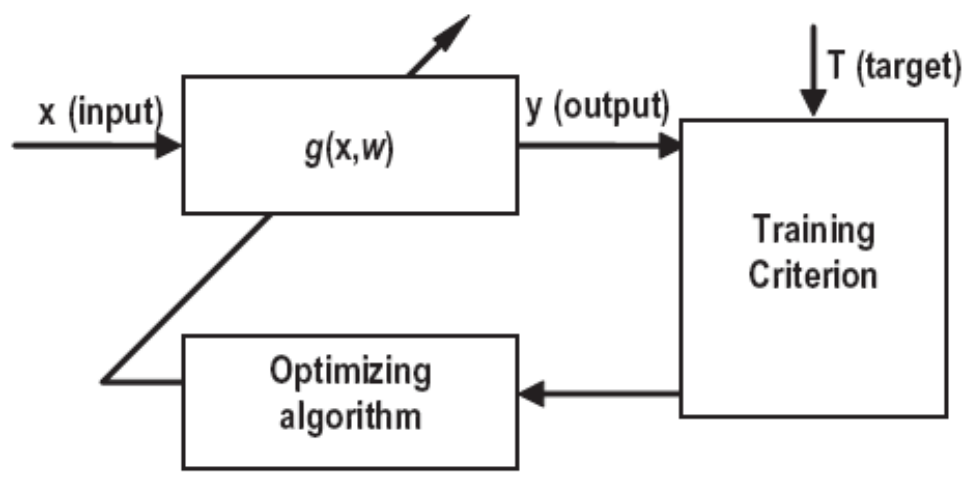

Fig. 2-1 Basic arrangement of a W2P identifying its three main modules.

\subsubsection{Training Criteria}

The traditional MSE and the mean absolute error (MAE) are training criteria with generalized adoption under the "forecaster's paradigm." Information theoretic learning (ITL) concepts especially challenge the unquestioned adoption of MSE, because this criterion is equivalent to minimizing the variance of the error distribution, and MSE is only an optimal criterion if this distribution is Gaussian - which is not true in most cases, especially not in WPF models.

Bessa et al. [37] first introduced the idea of exploring ITL criteria [38] to use in training neural networks for WPF (W2P model), where two training criteria from the ITL paradigm were considered. The first training criterion is the Minimum Error Entropy (MEE) [39]. The basic idea is as follows: if the error distribution of the output would become a Dirac function (meaning that all errors would be equal), we would have reached a predictor whose output would reproduce exactly the actual data series - by just adding to the results a bias corresponding to the mean of the probability density function ( $p d f)$ of the errors (i.e., the deviation from zero). But it so happens that the Dirac function has minimum entropy. Renyi's Entropy is combined with a Parzen Windows estimation of the error $p d f$ to form the basis of MEE under which neural networks are trained.

The second training criterion was derived from an ITL measure named correntropy [40]. Correntropy is a generalized similarity measure between two arbitrary scalar random variables, $\mathrm{X}$ and $\mathrm{Y}$, defined by:

$$
V_{\sigma}(X, Y)=E\left[k_{\sigma}(X-Y)\right]
$$

where $k_{\sigma}$ is the kernel function (usually Gaussian).

Correntropy is directly related to the probability of how similar two random variables are along the line $y=x$ in a neighborhood of the joint space defined by the kernel bandwidth $\sigma$, and it provides the probability density of the event $p(X=Y)$. The bandwidth controls the observation window in which the similarity is assessed but makes it impossible to assess similarity in the whole joint space. This limitation is actually good, because it leads to the rejection of outliers whose consideration contaminates models. The training criterion consists of maximizing the 
correntropy between the output $y$ and the target $T$ and was called Maximum Correntropy Criterion (MCC). These two ITL criteria were only studied in the context of the "forecaster's paradigm," and no attention was given to the value of the forecast.

Viewing the WPF problem from the "forecast user's paradigm," for a WGENCO, the training criterion should be translated into economic value, which is related with profit in the market and penalization of forecast errors. Hence, the cost function presented in (2-3) could in theory lead to a better "forecast" under this paradigm. An alternative that we propose is to use the parametric correntropy [41], which has been used so far in signal processing problems. The idea is similar to that of correntropy; however, the comparison between $\mathrm{X}$ and $\mathrm{Y}$ is performed along the line, $a X+b=y$, where $a$ and $b$ are parameters.

The training criteria that we use to train the W2P model (e.g., neural networks) in the WPF problem are summarized as follows:

1. MSE - Minimum Square Error. This value is the classical criterion that minimizes the variance of the error distribution and has the form:

$$
\operatorname{MSE}(e) \Leftrightarrow \min \frac{1}{N} \sum_{i=1}^{N} \frac{1}{2} e_{i}^{2}
$$

where $e=\left(T_{i}-y_{i}\right)$ is the error of sample $i$ relative to the target value $T_{i}$, and $N$ the number of training samples.

2. MCC - Maximum Correntropy Criterion. This criterion is based on a correntropy and may be expressed as:

$$
\operatorname{MCC}(e) \Leftrightarrow \max \frac{1}{N} \sum_{i=1}^{N} k_{\sigma}\left(e_{i}\right)
$$

where $k_{\sigma}$ is the Gaussian Kernel with bandwidth $\sigma$.

3. MAE - Minimum Absolute Error. This criterion minimizes the absolute error without taking into account the sign of the error:

$$
\operatorname{MAE}(e) \Leftrightarrow \min \frac{1}{N} \sum_{i=1}^{N}\left|e_{i}\right|
$$

4. MPC - Minimum Penalty Costs. This criterion minimizes the error penalties, as shown in $(2-3)$.

5. MPCC - Maximum Parametric Correntropy Criterion. This criterion is based on parametric correntropy and may be expressed as: 


$$
\operatorname{MPCC}(e, a) \Leftrightarrow \max \frac{1}{N} \sum_{i=1}^{N} k_{\sigma}\left(a \cdot y_{i}-T_{i}\right)
$$

where $a$ is a parameter that is defined by the forecaster or computed from the market balancing costs, and $b$ is set to zero because the bias of the forecast can be introduced only with parameter $a$, without increasing the number of parameters of the problem.

\subsection{Case Studies}

\subsubsection{Data Characteristics}

Three real wind farms with different rated power (ranging from $20 \mathrm{MW}$ to $50 \mathrm{MW}$ ) and situated in different types of terrain are used as case studies. Data collected in the wind farms include supervisory control and data acquisition registers with an average "package" containing 10 minutes of power delivered by the wind farms to the grid. We also have available forecasts produced for the same period by a mesoscale weather model for mean wind speed and wind direction for a reference point in the wind farm, with forecasting horizons ranging from 0 to 48 hours in 1-hour intervals. These data cannot be discussed in further detail for reasons of confidentiality.

To organize the tests, the available data were divided into three sets. The first set, with several months' worth of data, was used as a training set. The second set, with one month's worth of data, was used as a validation set. And the third set, with the remaining months, was used as a testing set for comparative purposes. Table 2-1 presents the months used for training, testing, and validation of the neural network.

Table 2-1 Training, validation, and testing sets.

\begin{tabular}{cccc}
\hline Wind Farm & Training & Validation & Testing \\
\hline \multirow{2}{*}{ A } & Feb-Dec & Jan (2007) & Jan-Dec \\
& $(2007)$ & & $(2008)$ \\
B & Mar-Dec & Feb (2007) & Apr-Dec \\
& $(2007)$ & & $(2008)$ \\
C & Feb-Aug & Jan (2007) & Sep-Dec \\
& $(2007)$ & & $(2007)$ \\
\hline
\end{tabular}

Data from the Iberian electricity market was used in this study. The data consists of hourly spot and balancing prices from two years, 2007 and 2008, and was collected from e-sios (the information system of the system operator of Red Eléctrica de Espãna, http://www.esios.ree.es). A very complete statistical analysis of the price data used in this study can be found in [42] (see Chapter 5). Moreover, the author analyzed the correlation between the market prices (spot and balancing) and the wind generation for the same data used in this chapter. The author concluded that wind power is still having only a limited impact on the spot prices in the Iberian market; however, it has already made an impact on the balancing prices. Table 2-2 shows the annual mean energy prices in 2007 and 2008 for the Spanish market (in €/MWh). 
Table 2-2 Annual mean market prices.

\begin{tabular}{ccc}
\hline & 2007 & 2008 \\
\hline Spot Price & 39.34 & 64.47 \\
Up Balancing & 2.97 & 4.04 \\
Down Balancing & 7.99 & 8.02 \\
\hline
\end{tabular}

The balancing prices are very asymmetric, where the down balancing is more expensive. Also evident is a considerable change in prices from one year to the next, but this variation does not affect the results of our study — what is relevant is the asymmetry in balancing prices.

\subsubsection{Training Characteristics}

We have trained a feed-forward multilayer perceptron (MLP) neural network with only one hidden layer consisting of 7 neurons by using a hyperbolic tangent activation function. The inputs of the MLP are NWP forecasted values: mean wind speed values and mean wind direction values. Because of the cyclic character of the wind direction, this variable comprised two components (i.e., the sine and cosine components). This means a total of three input variables, which were standardized by using the min-max method, were used.

Because the purpose is to compare results, we do not describe details of the training here; however, the training details can be found in [37]. Moreover, determining the best topology of the neural network is beyond the scope of this work.

The penalization factors of the training criterion MPC (2-3) consist in average values of balancing prices for each look-ahead time step (in this case, there are 24 time steps) computed from year 2007 data. Hence, the diurnal cycle of the prices is included in the model. Regarding the parameter $a$ of the MPCC criterion, the adopted methodology is:

1. In the beginning of each training epoch, the parameter $a$ is estimated from the training dataset by using the average value of balancing prices of one year for each look-ahead time step as penalization factors of underestimation $\left(w_{\text {down } r e g}\right)$ and overestimation $\left(w_{\text {up_reg }}\right)$. The training criterion is:

$$
\max _{a}\left\{\begin{array}{c}
k_{\sigma^{\prime}}\left(\left(a \cdot y_{i}-T_{i}\right) \cdot w_{\text {down_reg }}\right), y_{i}<T_{i} \\
k_{\sigma^{\prime}}\left(\left(a \cdot y_{i}-T_{i}\right) \cdot w_{\text {up_reg }}\right), y_{i}>T_{i}
\end{array}\right.
$$

where $\sigma^{\prime}$ is the kernel bandwidth and depends on the size of the penalization factors.

2. After estimating the parameter $a$, the following training criterion is maximized:

$$
\max _{w} k_{\sigma}\left(a^{\prime} \cdot y_{i}-T_{i}\right)
$$

where $\sigma$ is a kernel bandwidth much smaller than $\sigma^{\prime}$, and $a^{\prime}$ is equal to $1 / a$. 
The kernel size $\sigma$ used by MCC is the same size used in [37]. The kernel size $\sigma$ in MPCC was found in the validation set to be equal to 0.1 , and $\sigma$ ' was equal to 3 . As expected, the kernel size does not vary widely from one wind farm to another. Yet, $\sigma^{\prime}$ presents a major importance in this problem, because small values indicate small sensitiveness to asymmetric costs. What is desired is a value that gives importance to the asymmetric costs but that also maintains a balance between over- and underestimation. The same values are used for the three wind farms since what is desired in WPF is to train a neural network for any wind farm without concern about tuning parameters.

\subsubsection{Testing Methodology}

The neural network was used to predict the power $\mathrm{p}_{\mathrm{t}+\mathrm{k} \mid \mathrm{t}}$ produced by the wind farm at time stamp 7:00 GMT (when new NWP predictions become available) for each look-ahead $t+k$ of the next day. The wind power prediction was performed for each day of the test dataset. We have taken each wind farm and simulated its participation in the electricity market in the year 2008, offering the power prediction generated by prediction systems trained with different training criteria and using the hourly spot and balancing prices from 2008.

\subsubsection{Analysis of the Results}

In this section, we present results for training of neural networks in three real wind farms and one electricity market, when training under the different criteria. Table 2-3 through Table 2-5 summarize the results obtained from the participation of wind farms $\mathrm{A}, \mathrm{B}$, and $\mathrm{C}$ in the Spanish market with bids equal to the forecasts provided by each training criterion. $\mathrm{Q}_{95} \%$ is the quantile $95 \%$ of the overestimation errors distribution, which means that the probability of having an upward deviation larger or equal to this value is $5 \%$; the conditional tail expectation (CTE) of this quantile can be interpreted as the expected maximum forecast deviation.

Table 2-3 Simulation of wind farm A's participation in the Iberian market.

\begin{tabular}{lccccc}
\hline & MSE & MCC & MAE & MPC & MPCC \\
\hline Surplus [\% of produced energy] & 33.79 & 35.55 & 39.08 & 19.71 & 30.89 \\
Shortage [\% of produced energy] & 33.74 & 29.64 & 24.37 & 58.07 & 35.56 \\
Total deviations [\% of produced energy] & 67.54 & 65.21 & 63.47 & 77.79 & 66.46 \\
\hline Deviations against the system [\% of produced energy] & 30.09 & 28.68 & 28.21 & 34.97 & 29.02 \\
Q95\% [\% of rated power] & 41.74 & 41.70 & 43.58 & 61.50 & 48.30 \\
CTE $^{\text {[\% of rated power] }}$ & 53.67 & 58.05 & 57.89 & 73.28 & 63.26 \\
\hline Total Income (deviation from the best) [k€] & -10.16 & -7.61 & -12.47 & -4.1 & 0.0 \\
${ }^{a}$ CTE $=$ conditional tail expectation. & & & & &
\end{tabular}

Table 2-4 Simulation of wind farm B's participation in the lberian market.

\begin{tabular}{|c|c|c|c|c|c|}
\hline & MSE & $\mathrm{MCC}$ & MAE & MPC & MPCC \\
\hline Surplus [\% of produced energy] & 34.09 & 34.13 & 39.29 & 18.21 & 25.85 \\
\hline Shortage [\% of produced energy] & 25.16 & 24.14 & 19.67 & 48.31 & 34.37 \\
\hline Total deviations [ $\%$ of produced energy] & 59.25 & 58.28 & 58.96 & 66.52 & 60.23 \\
\hline Deviations against the system [\% of produced en & 26.33 & 26.10 & 26.41 & 28.83 & 26.49 \\
\hline Q95\% [\% of rated power $]$ & 28.19 & 30.22 & 26.98 & 40.67 & 32.92 \\
\hline $\mathrm{CTE}^{\mathrm{a}}[\%$ of rated power] & 34.21 & 40.27 & 34.78 & 49.78 & 42.13 \\
\hline Total Income (deviation from the best) [k€] & -20.09 & -20.43 & -30.92 & 0.0 & -7.45 \\
\hline
\end{tabular}


${ }^{\mathrm{a}} \mathrm{CTE}=$ conditional tail expectation.

Table 2-5 Simulation of wind farm C's participation in the lberian market.

\begin{tabular}{llllll}
\hline & MSE & MCC & MAE & MPC & MPCC \\
\hline Surplus [\% of produced energy] & 34.46 & 36.91 & 37.63 & 19.58 & 24.62 \\
Shortage [\% of produced energy] & 19.71 & 16.09 & 15.02 & 33.12 & 27.42 \\
Total deviations [\% of produced energy] & 54.17 & 53.00 & 52.64 & 52.70 & 52.04 \\
\hline Deviations against the system [\% of produced energy] & 23.07 & 22.88 & 23.14 & 20.54 & 21.13 \\
Q95\% [\% of rated power] & 31.08 & 34.00 & 38.34 & 46.07 & 39.44 \\
CTE $^{\mathrm{a}}$ [\% of rated power] & 38.33 & 44.42 & 46.88 & 52.42 & 48.21 \\
\hline Total Income (deviation from the best) [k€] & -14.2 & -15.35 & -16.59 & 0.0 & -4.78 \\
\hline
\end{tabular}

${ }^{\mathrm{a}} \mathrm{CTE}=$ conditional tail expectation.

Wind Generation Companies' Viewpoint

The first three training criteria are not economically oriented. One may see that wind farm A realized a lower level of income when using them than it did with MPC and MPCC. The comparison of the results from MPC with the first three training criteria (which are typical of the forecaster paradigm) illustrates that a forecast of worse quality (i.e., larger deviations) results nevertheless in higher income for a WGENCO. By comparing MCC with MPCC, it is evident that accepting a small increment in the error leads to an increase of around 7,600 $€$ in revenue. In addition, for wind farm $\mathrm{A}$ and over the one-year test period, the forecasts generated by the MPCC led to the highest profits (around 4,100€ higher) than did those forecasts obtained with MPC.

For wind farm B, the first three training criteria also led to lower income levels. In this case, MPC presents a higher profit potential than does MPCC (by around 7,500€). The same conclusions for wind farm $\mathrm{B}$ are also valid for wind farm $\mathrm{C}$. The economic-oriented training criteria led to higher levels of income, and once more the MPC realized a higher level of income than did the MPCC.

From the results of the three wind farms, it becomes clear that using MPCC instead of any statistical criterion enables increases to the income without significantly increasing the deviations resulting from forecast errors.

\section{System Operator Viewpoint}

For wind farm A, the forecasts generated with MCC and MAE produce fewer total deviations and deviations against the system. MCC and MAE underestimate the wind generation, but despite this underestimation, a higher income was achieved with MCC when compared with MSE. The MSE criterion places a heavy penalty on large errors, and therefore the CTE for MSE presents the lowest value of all of the training criteria - this result means that wind farm A is usually perceived as a good property by the SOs. It is therefore satisfactory that the new criterion, MPCC, could lead as well to a high level of income without significantly increasing the total deviations and the deviations against the system. On the other hand, the MPC has a higher bias on the side with lower balancing costs (e.g., a shortage of generation), which is in opposition to the interests of the SO in terms of security of the system. The MPCC for wind farm A is near the "compromise viewpoint," as it allows some increase in the income without a major increase 
in the total deviation. The comparison of MPC and MPCC in the quantile 95\% and CTE values also show that MPCC is better for the SO when compared with MPC.

For wind farm B, the first three training criteria led to lower total deviations and deviations against the system. The tendency for this wind farm is to underestimate the wind generation. For this wind farm, the training criterion with fewer total deviations was MCC, which is surprising because MAE was expected to achieve fewer total deviations. Once more, MSE presents the lowest value of CTE. MPC presents a higher realized income than does MPCC; however, MPCC allowed (or enabled) increases in realized income while the total number of deviations and deviations against the system are maintained at an acceptable level.

The same behavior is verified for wind farm $\mathrm{C}$, where, while the MPCC realized a lower income than did the MPC, the total deviation values and CTE are lower. In this case, MPC presented a lower value for deviations against the system.

\subsection{Conclusions}

This chapter addresses the WPF problem by recognizing that the value and quality of forecasts must be appreciated in the framework that decision making is carried out by multiple agents and that agreement may not be possible among the agents (WGENCOs or SOs) regarding what constitutes a "good" forecast. The issues discussed - the relation between forecast error and market profit - also occur in situations involving more complex forecasting systems and electricity market rules. The results in this chapter provide evidence that forecasts with higher accuracy (in the forecaster's paradigm sense) do not necessarily lead to higher incomes for a WGENCO. As such, a WGENCO may be willing to reduce the forecasts' accuracy in exchange for an increased income.

The main contributions of the chapter are summarized in the following:

1. A challenge to the assumption of the mathematical neutrality of forecasting models.

2. A thorough discussion of conflicting interests associated with the "goodness" of forecasts for two types of agents in an electricity market.

3. A comparison of the relative advantages and drawbacks of several criteria referred to in the literature and used in training WPF models.

4. A new approach proposed to achieve acceptable "compromise" solutions to market participants in the choice of a forecasting model by introducing the ITL concept of parametric correntropy.

5. The verification of the theoretical concepts in a representative example, namely, the Iberian market, MIBEL.

What is the significance of the results shown in this chapter for future forecasting practices and market policies? First, these results reveal the importance of the choice of a training criterion for a forecasting tool. In this sense, it is not necessarily true that it is impossible to achieve a high remuneration in the electricity market and maintain the forecast error within an acceptable level. Methods that produce acceptable compromises are available. Second, in systems with high wind penetration levels, it is important to look at the impact of the wind generation market bids on the balancing needs. In fact, the market rules and the way that wind power is remunerated in the market should be revisited and possibly redesigned to better facilitate the decisions made by the 
SO regarding the security of the system. This chapter shows that an asymmetry in balancing prices makes possible the opportunity for profit by those forecaster's paradigm WGENCOs that make careful selection of the characteristics of the output of the forecasting systems. Whenever there is an asymmetry in the price structure, whether in balancing prices or spot prices (which is more likely to occur in cases of large penetration of wind power, with larger impacts on price volatility), a neutral model (in the forecaster's paradigm sense) may not be the model that leads to maximizing the profits of WGENCOs that bid in the market.

This chapter also points toward areas for wider discussion, namely, toward an analysis on possible reactions of other agents in the market to wind power bids, especially if indications appear that bids do not correspond to a "neutral" forecast (in the forecaster paradigm sense), which could lead to speculation against admittedly biased wind power forecasts. One possible evolution that the results in this chapter suggest would be to decouple the concepts of forecasting and bidding. In some markets these days, SOs demand that WGENCOs bid the forecast they have produced - and then trust it as a valid forecast. However, WGENCOs may wish to develop a bidding strategy, and this impetus leads to the possibility of adopting models that, while seeming to be mathematically "neutral," will have characteristics that serve their particular market strategy - and will possibly be contrary to SOs' interests. So, it follows naturally that WGENCOs may be allowed to bid in an unrestricted manner on whatever power value they prefer, but that SOs will also have their own independent forecasts and will act according to those and not solely to the forecasts announced by WGENCOs in order to ensure reliability in the power system. 


\section{WIND POWER FORECASTING IN U.S. ELECTRICITY MARKETS ${ }^{2}$}

WPF is becoming an important tool in electricity markets as the amount of wind power rapidly increases. However, the use of WPF in market operations and among market participants is still at an early stage. We discuss the current use of WPF in U.S. ISO/RTO markets, and give recommendations for how to make efficient use of the information in state-of-the-art wind power forecasts.

\subsection{Introduction}

Wind power generation has increased rapidly in the United States over the last few years, and at the end of 2010, there was an installed capacity of more than $40,000 \mathrm{MW}$ at a national level. ${ }^{3}$ Wind power is already having a significant impact on the operation of electricity markets and power systems in areas with the highest penetration of wind power, such as in the Electric Reliability Council of Texas (ERCOT) and the Midwest ISO (MISO). A number of challenges arise when integrating renewable generation into the power system, from transmission planning, resource adequacy, and interconnection standards to dealing with the increased uncertainty and variability in short-term operations. Many of these challenges are discussed in recent reports by the North American Electric Reliability Council (NERC) [43] and the U.S. Department of Energy [44].

It is generally agreed that forecasting will play an increasingly important role in the operation of power systems as the level of wind power increases. WPF models have been continuously improving over the last decade. A number of WPF providers have emerged that are competing to provide the best forecasts to the electric power industry. There are several different users of the wind power forecasts, including generation companies, wind power producers, utilities, market analysts and traders, and power system and electricity market operators. The desired forecasting capabilities typically vary among user groups. For instance, system operators are particularly concerned with the ability to forecast rapid changes in wind power production, so-called ramping events. Wind power producers, on the other hand, may be more concerned with minimizing their forecasting error over a longer time period to better schedule their resources in the marketplace. Some companies already have considerable experience in the use of WPF, as documented in $[7][45][46]$.

In this chapter, we first give a brief introduction to WPF models. We then present an overview of the current use of WPF in U.S. electricity markets, with focus on the ISO/RTO markets that currently have the highest penetration of wind power. Finally, we discuss the need to revise market design to make better use of the information provided by advanced WPF systems in market operations.

\subsection{Wind Power Forecasting Models}

A WPF system, as illustrated in Fig. 1-1, uses input data from different sources, including results from numerical weather prediction (NWP) models, local meteorological measurements, SCADA

\footnotetext{
${ }^{2}$ This chapter is reproduced from the Electricity Journal, Vol. 23, No. 3, A. Botterud, J. Wang, V. Miranda, and R.J. Bessa, "Wind Power Forecasting in U.S. Electricity Markets," pp. 71-82, 2010, with permission from Elsevier.

${ }^{3}$ According to the American Wind Energy Association (AWEA), http://www.awea.org.
} 
data describing the real-time (RT) state of the wind power plants, and additional information about the characteristics of the wind power plants and the nearby terrain and topography. Modern WPF models are usually based on a combination of physical and statistical models. The physical approach describes the physical relationship between atmospheric conditions, local topography, wind speed, and the output from the wind power plant. In contrast, the statistical approach estimates a statistical relationship between all of the relevant input data and the resulting wind power generation without considering the physics of the system. The performance of wind power forecasts and the forecast accuracy depends on the availability of good NWP forecasts, the complexity of the terrain, and the availability of RT weather and wind power plant data. Hence, there can be large differences in forecasting errors between wind power plants at different locations. WPF systems typically produce forecasts for a time horizon up to 2-3 days ahead in time. In general, the forecasting error increases with the forecast horizon.

WPF models are continuously being improved with the goal of increasing the forecast accuracy. Ongoing research also focuses on producing probabilistic forecasts, because information about the uncertainty in the wind generation can be of great value for system operators and market participants. As an example, Fig. 3-1 shows two different types of uncertainty representation in WPF. Ramp forecasting is another important area of research. For a detailed review of the current state of the art in WPF, we refer to our state-of-the-art report [1].
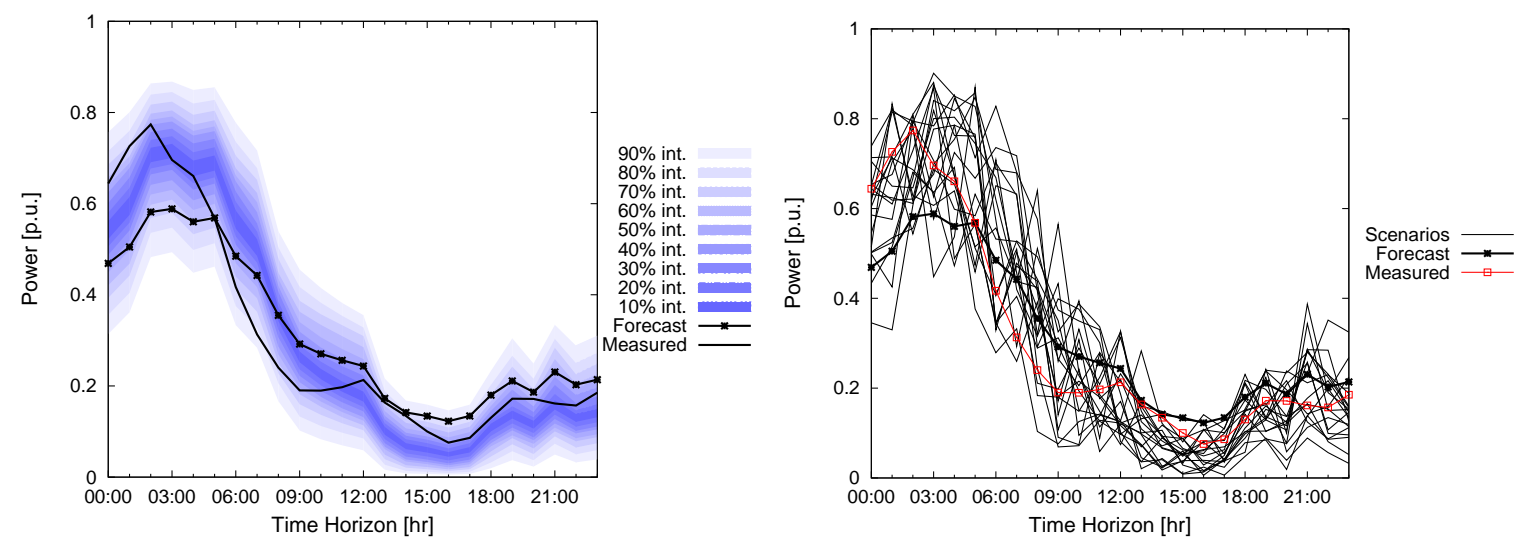

Fig. 3-1 Uncertainty representation in wind power forecasts: (a) probabilistically by a set of intervals or quantiles (left), and (b) scenarios of wind power generation representing the temporal dependency of forecast errors (right).

\subsection{Use of Forecasting in Market Operations}

In this section, we discuss the potential use of WPF in the short-term operation of ISO/RTO markets in the United States. The markets in different regions of the country have seen a considerable degree of convergence in market design in recent years. ISO/RTO markets already have several features that are advantageous for the integration of wind power into the electric power system, such as the following:

1. Large Balancing Authority Areas give the system operator the control of generation and demand resources over a larger area, increasing the overall flexibility of the system to handle variations in wind power generation. At the same time, the variation in total wind 
power generation, and also the WPF error, generally decreases as a function of geographical size. Each ISO/RTO is operated as one balancing authority area.

2. Centralized unit commitment and scheduling of energy and operating reserves gives the system operator control of a large pool of generation and load resources, which can be used to effectively accommodate fluctuations in wind power output. Combined operation of energy and operating reserves markets also ensures cost-efficient and market-based procurement of energy and operating reserves.

3. Frequent dispatch of resources in real time. Most ISO/RTO markets re-dispatch the system resources every five minutes, upon which new set-points are sent to generators and eligible demand resources. The frequent system dispatch reduces the need to maintain expensive regulation reserves to respond to short-term fluctuations in load and non-dispatchable generation.

4. Locational marginal prices (LMPS) give locational market-based incentives for shortterm operation and long-term expansion. LMPs can also enable price-based and efficient curtailment of wind generation during periods with surplus wind power generation, which are typically caused by a combination of transmission bottlenecks and minimum generation limits for thermal power plants. LMPs also provide efficient incentives for location of new wind power generation, taking network congestion into account.

The experience with WPF, however, is relatively limited so far. A brief summary of the current state of WPF for MISO, New York ISO (NYISO), PJM, ERCOT, and California ISO (CAISO) is provided in Table 3-1. A typical timeline for the operation of the electricity market is shown in Fig. 3-2. The procedures and timeline in the figure are based on the current rules in the MISO market. However, other ISO/RTO markets are operated in a similar way. The main steps in the market operations, including determination of reserve requirements, DA operations, reliability assessment commitment, and RT operations, are discussed below. As indicated in Fig. 3-2, WPF can play an important role in all of these steps as wind power penetration increases.

\subsubsection{Operating Reserve Requirements}

The requirements for operating reserves in U.S. power systems are based on standards determined by the NERC [47]. Operating reserves are categorized into several types depending on how quickly they can respond to changes in the system (Fig. 3-3). The regulating reserve responds immediately to balancing needs in the system, and is usually provided by generating units with automatic generation control (AGC) responding to frequency deviations in the network.

Contingency reserves are used to respond to contingencies that may occur, such as forced outages of generators or transmission lines. The contingency reserve can be split into spinning and non-spinning reserves, where the spinning component needs to be able to respond within 10 minutes. It is common that both generation and demand resources can provide contingency reserves. In the event that operating reserves need to be used due to a contingency in the system, the required level of reserve must be restored as soon as possible and at least within 60 minutes [47]. 
The ISO/RTOs are required to maintain sufficient regulating reserve to meet NERC's criterion for area control error. ${ }^{4}$ The requirement for contingency reserves is based on the N-1 rule, that is, that sufficient contingency reserve must be held to cover "the loss of generating capacity due to forced outages of generation or transmission equipment that would result from the most severe single contingency." At least half of the contingency reserve must be spinning. These are the minimum requirements set by NERC; however, regional variations exist and some ISO/RTOs use more stringent requirements for their operating reserves. In systems with large and congested networks, it is common to specify regional reserve requirements in addition to the system-wide criteria. It is also interesting to note that some markets have introduced a demand curve for different types of operating reserves rather than the traditional fixed requirements. Operating reserve requirements may be updated to accommodate changes in the system conditions. Although the update frequency varies between different markets (seasonal, monthly, and daily updating), the requirements for the next operating day must be posted at least before operation of the DA market begins.

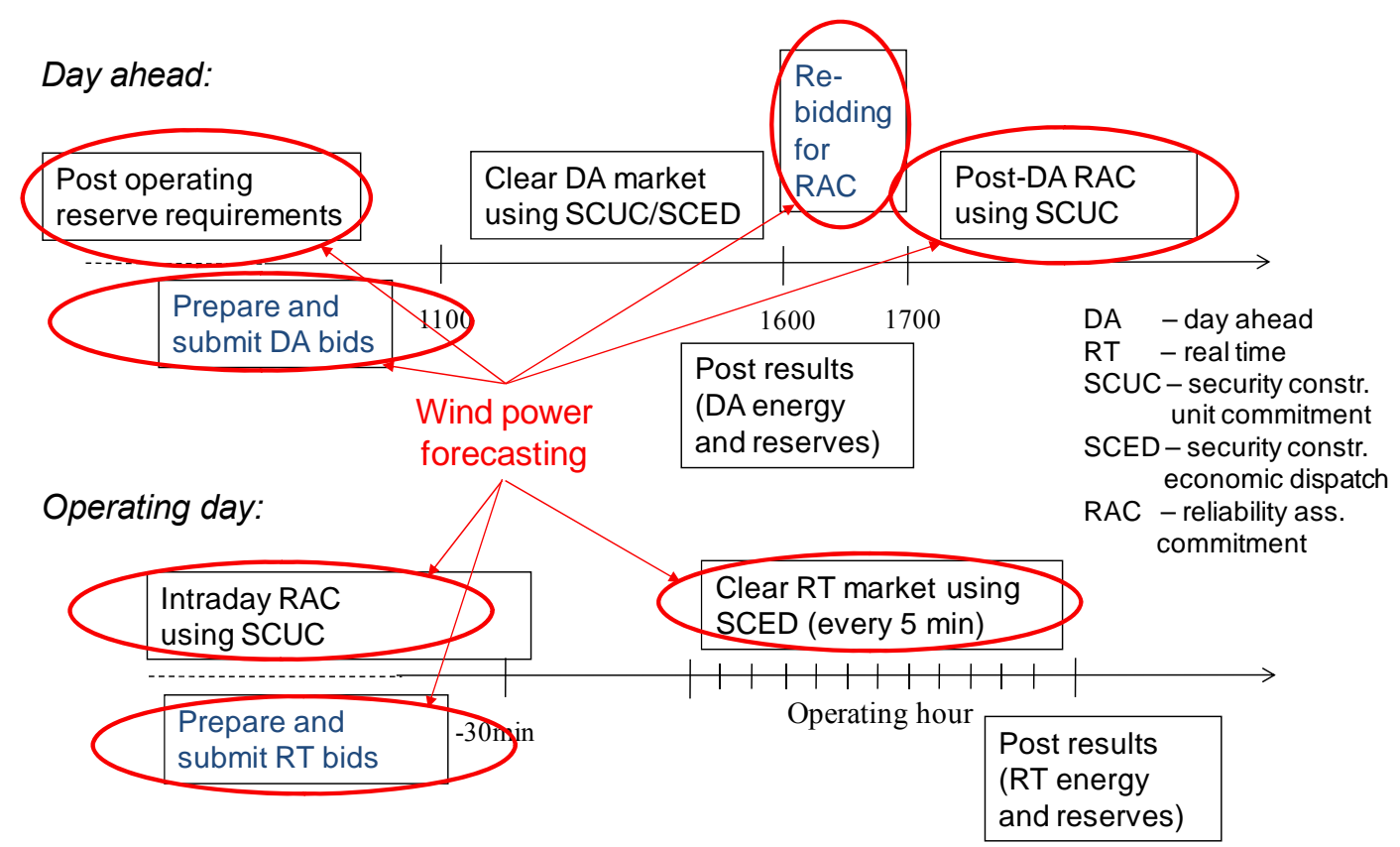

Fig. 3-2 Market operations timeline for MISO, indicating where WPF could play an important role.

\footnotetext{
${ }^{4}$ The Area Control Error is a measure for the deviation between scheduled and realized exchange from a balancing authority area and is defined by NERC as "the instantaneous difference between net actual and scheduled interchange, taking into account the effects of Frequency Bias including correction for meter error."
} 
Table 3-1 Overview of WPF and market operation in five U.S. electricity markets. Based on information from the Utility Wind Integration Group (UWIG) and ISO/RTOs.

\begin{tabular}{|c|c|c|c|c|c|}
\hline & MISO & NYISO & PJM & ERCOT & CAISO \\
\hline Peak load & $\begin{array}{l}\text { 116,030 MW } \\
(7 / 31 / 2006)\end{array}$ & \begin{tabular}{|l|}
$33,939 \mathrm{MW}$ \\
$(8 / 2 / 2006)$ \\
\end{tabular} & $\begin{array}{l}\text { 144,644 MW } \\
(8 / 2 / 2006)\end{array}$ & $\begin{array}{l}62,339 \mathrm{MW} \\
(8 / 17 / 2006) \\
\end{array}$ & \begin{tabular}{|l|}
$50,270 \mathrm{MW}$ \\
$(7 / 24 / 2006)$ \\
\end{tabular} \\
\hline $\begin{array}{l}\text { Total } \\
\text { installed } \\
\text { capacity }\end{array}$ & Ca. $138,000 \mathrm{MW}$ & Ca. 38,000 MW & $\begin{array}{l}\text { Ca. 165,000 } \\
\text { MW }\end{array}$ & Ca. $80,000 \mathrm{MW}$ & $\begin{array}{l}\text { Ca. 59,000 MW } \\
\text { (incl. imports) }\end{array}$ \\
\hline $\begin{array}{l}\text { Wind } \\
\text { capacity } \\
\text { (end of 2009) }\end{array}$ & Ca. 7,600 MW & Ca. $1,300 \mathrm{MW}$ & Ca. 2,500 MW & Ca. 9,000 MW & Ca. 3,000 MW \\
\hline $\begin{array}{l}\text { Wind power } \\
\text { forecasting }\end{array}$ & $\begin{array}{l}\text { In operation since } \\
\text { 2008: } \\
\text { - DA and intra- } \\
\text { day RAC } \\
\text { - Transmission } \\
\text { security and } \\
\text { outage } \\
\text { coordination } \\
\text { - Transmission } \\
\text { security and } \\
\text { peak load } \\
\text { analysis } \\
\text { - Indication of } \\
\text { ramps }\end{array}$ & $\begin{array}{l}\text { In operation since } \\
\text { 2008: } \\
\text { - Reliability } \\
\text { assessment } \\
\text { commitment at } \\
\text { DA stage } \\
\text { - RT } \\
\text { commitment } \\
\text { and dispatch } \\
\text { - Ramping alert } \\
\text { system under } \\
\text { consideration }\end{array}$ & $\begin{array}{l}\text { In operation } \\
\text { since 2009: } \\
\text { - DA } \\
\text { transmission } \\
\text { security and } \\
\text { reserve } \\
\text { adequacy } \\
\text { assessments } \\
\text { - Developing } \\
\text { automated } \\
\text { procedures } \\
\text { - Specific ramp } \\
\text { forecast }\end{array}$ & $\begin{array}{l}\text { In operation } \\
\text { since } 2008: \\
\text { - } 80 \% \\
\text { exceedance } \\
\text { forecast used } \\
\text { for DA } \\
\text { planning } \\
\text { - To be fully } \\
\text { integrated in } \\
\text { new nodal } \\
\text { design, to be } \\
\text { introduced } \\
\text { end of 2010 } \\
\text { - Developing } \\
\text { ramp forecast }\end{array}$ & $\begin{array}{l}\text { In operation } \\
\text { since 2004: } \\
\text { - Used to } \\
\text { calculate } \\
\text { energy } \\
\text { schedule in RT } \\
\text { market } \\
\text { - Advisory role } \\
\text { in DA market }\end{array}$ \\
\hline $\begin{array}{l}\text { Market } \\
\text { timeline }\end{array}$ & $\begin{array}{l}\text { DA bids due: } \\
11 \text { a.m. } \\
\text { DA results: } 4 \text { p.m. } \\
\text { Re-bidding due: } \\
5 \text { p.m. } \\
\text { RT bids due: } \\
\text { OH }-30 \text { min }\end{array}$ & $\begin{array}{l}\text { DA bids due: } \\
5 \text { a.m. } \\
\text { DA results: } \\
11 \text { a.m. } \\
\text { RT bids due: } \mathrm{OH} \\
-75 \text { min }\end{array}$ & $\begin{array}{l}\text { DA bids due: } \\
\text { noon } \\
\text { DA results: } \\
4 \text { p.m. } \\
\text { RT bids due: } \\
6 \text { p.m. (DA) }\end{array}$ & $\begin{array}{l}\text { DA bids due } \\
\text { (reserves only): } \\
1 \text { p.m./4 p.m. } \\
\text { DA results: } \\
\text { 1:30 p.m./6 p.m. } \\
\text { RT bids due: } \\
\text { OH -60 min }\end{array}$ & $\begin{array}{l}\text { DA bids due: } \\
10 \text { a.m. } \\
\text { DA results: } \\
1 \text { p.m. } \\
\text { RT bids: } \mathrm{OH} \mathrm{-} \\
75 \text { min }\end{array}$ \\
\hline $\begin{array}{l}\text { Wind power } \\
\text { bidding, } \\
\text { dispatch, } \\
\text { imbalance } \\
\text { settlements, } \\
\text { deviation } \\
\text { penalties }\end{array}$ & $\begin{array}{l}\text { - If wind is a } \\
\text { capacity } \\
\text { resource, it must } \\
\text { bid in DA } \\
\text { market and RAC } \\
\text { - No deviation } \\
\text { penalties } \\
\text { - No wind } \\
\text { dispatch, but this } \\
\text { is being } \\
\text { considered }\end{array}$ & $\begin{array}{l}\text { - Wind required } \\
\text { to bid in RT } \\
\text { market. DA } \\
\text { bidding } \\
\text { optional } \\
\text { - Dispatch } \\
\text { signals } \\
\text { provided from } \\
\text { SCED } \\
\text { - Penalty for } \\
\text { over-generation } \\
\text { in constrained } \\
\text { situations } \\
\text { - No penalties } \\
\text { for under- } \\
\text { generation }\end{array}$ & $\begin{array}{l}\text { - If wind is a } \\
\text { capacity } \\
\text { resource, it } \\
\text { must bid in DA } \\
\text { market } \\
\text { - Deviation } \\
\text { charges apply } \\
\text { - Wind dispatch } \\
\text { signals } \\
\text { provided in } \\
\text { constrained } \\
\text { situations }\end{array}$ & $\begin{array}{l}\text { - Bilateral } \\
\text { market } \\
\text { - Imbalances } \\
\text { settled at RT } \\
\text { zonal energy } \\
\text { price } \\
\text { - Penalty } \\
\text { exemption for } \\
+/-50 \% \text { of } \\
\text { scheduled } \\
\text { generation } \\
\text { - Ramping } \\
\text { limits }\end{array}$ & $\begin{array}{l}\text { - Wind program } \\
\text { participants' } \\
\text { RT bid based } \\
\text { on ISO } \\
\text { forecast. } \\
\text { Deviations } \\
\text { netted over } \\
\text { month at } \\
\text { average price } \\
\text { - 10-min } \\
\text { imbalance } \\
\text { charges for } \\
\text { nonparticipants } \\
\text { - Wind is not } \\
\text { dispatched }\end{array}$ \\
\hline
\end{tabular}

Key: $\mathrm{DA}=$ day-ahead, $\mathrm{OH}=$ operating hour, $\mathrm{RAC}=$ reliability assessment commitment, $\mathrm{RT}=$ real-time, $\mathrm{SCED}=$ security-constrained economic dispatch 


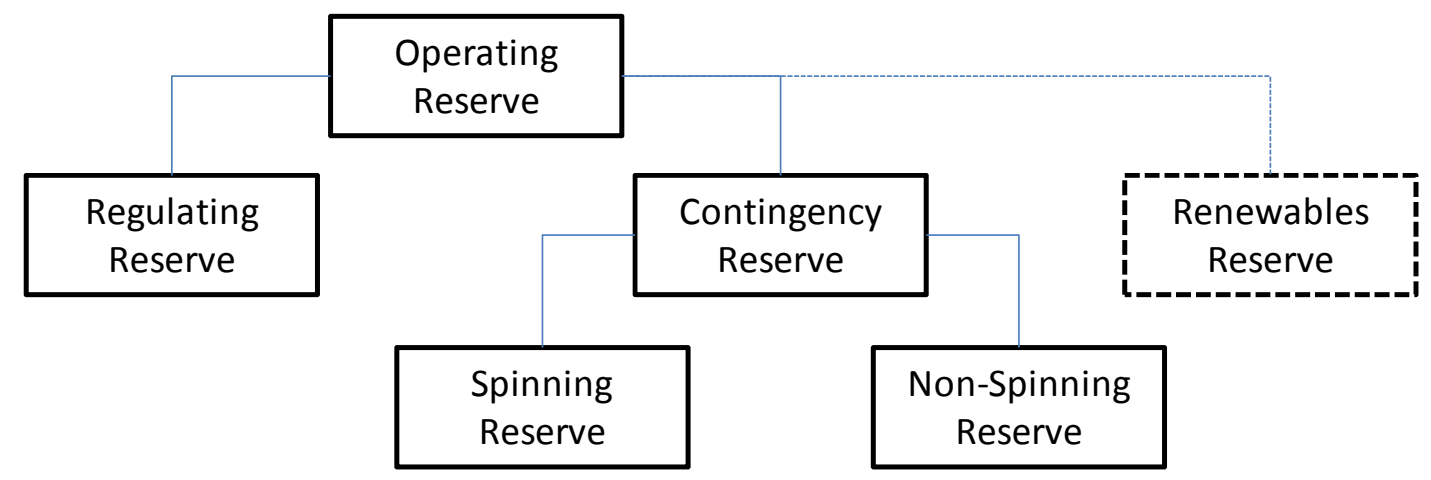

Fig. 3-3 Traditional operating reserve categories as defined by NERC and a proposed new category to address increased uncertainty and variability from renewable generation.

The additional uncertainty and variability caused by an increasing penetration of wind power generation raises the question of whether current requirements for operating reserves are adequate. In general, WPF can guide the procurement and use of the operating reserves by increasing the system operators' knowledge of projected wind power generation and therefore the overall balance between supply and demand in the system. Several improvements to existing requirements and procedures are relevant, including:

1. New definitions of operating reserve requirements that better reflect the needs in power systems with a large share of renewable energy. For instance, changes in wind power output unfold much more slowly than the traditional contingencies, which are caused by sudden equipment failures. Hence, the system operator has more time to respond to these changes, and a slower type of reserve than the current contingency reserves (at least slower than the current spinning reserves) may be sufficient. At the same time, it is less critical to replace these reserves immediately, as a second down-ramp event is not likely, and oftentimes not even possible, if there has already been one major reduction in wind power generation. An additional type of "renewables reserve" is therefore proposed in Fig. 3-3. Such a reserve category would have slower response and restoration times and so should be less expensive than the traditional contingency reserves. There is also likely to be a need for additional regulation reserves. However, the short dispatch intervals reduce the need for regulation to handle short-term variability in wind generation. Better short-term wind power forecasts would also limit the amount of regulation reserves needed.

2. Improved determination of reserve requirements to better accommodate the characteristics of wind power and other renewable generation. Traditional deterministic criteria (e.g., loss of the largest unit in the system) should be replaced with probabilistic approaches that directly reflect the uncertain nature of supply and demand. Ideally, probabilistic wind power forecasts should be used in the calculation of improved reserve requirements that account for the risk of losing load due to wind generation forecast errors in addition to forced outages and unexpected changes in load. The historical forecasting errors should also be taken into account. Ongoing research looks at probabilistic determination of optimal spinning reserve criteria [30][48] in systems with high wind power capacity, as well as demand curves for operating reserves [49]. 
3. Dynamic and frequent updating of operating reserve requirements based on the most updated system information, including the most recent wind power forecast. This will result in better-informed decisions, and thus in more efficient operations. More frequent updating of reserve requirements would enable operating reserves to be adjusted according to how much wind generation is forecasted for the next hours and days, as well as the projected uncertainty in the forecast.

Several ISO/RTOs are currently considering the need to change their procedures and requirements for operating reserves. ERCOT, which is the ISO/RTO with the highest level of wind power capacity in the United States (Table 3-1), is already considering wind power penetration and forecasting uncertainty in their determination of requirements for regulation and non-spinning reserves [50]. In a recent study of the ancillary services requirements in ERCOT [51], GE recommends that ERCOT make adjustments to its procedures as the level of wind power generation increases, pointing out the importance of making better use of forecasting in the procurement of different types of operating reserves, and that the level of reserves should be adapted to actual conditions.

\subsubsection{Day-Ahead Operations}

The deadline for market participants to bid into the DA market varies among the different ISO/RTOs, as shown in Table 3-1. The bids of the market participants must reflect how much energy and operating reserves they can provide. Information on unit commitment (UC) constraints (e.g., ramping rates, startup costs/times, minimum down-times, etc., for generating units), self-scheduling, and bilateral schedules is also provided to the ISO/RTO.

The clearing of the DA market is a two-stage procedure in most markets. First, a security constrained unit commitment (SCUC) is run to commit resources. Second, a security-constrained economic dispatch (SCED) algorithm is run based on the commitment schedule from the SCUC. Transmission constraints may be simplified or omitted from the SCUC formulation to solve the problem in a reasonable time. In contrast, the transmission constraints are always included in the SCED formulation, although usually with a simplified linear representation (DC-OPF). In most U.S. markets, the SCUC/SCED procedure co-optimizes energy and operating reserves. The output from the DA market clearing therefore includes schedules for energy and operating reserves. In addition, LMPs are derived for each transmission node, and market-clearing prices are also calculated for each category of operating reserves.

In the United States, about $70 \%$ of the installed wind power through 2008 was sold through Power Purchasing Agreements (PPAs), mainly to utilities [52]. Most of the wind power generation is therefore not being bid directly into the DA market by the wind power producers, but rather is integrated into the utilities' scheduling plans, along with loads and other generating resources. It is therefore hard to estimate to what extent wind power forecasts are taken into account in the bids and schedules submitted into the DA market. In many cases, wind power generation is first being dispatched in the RT market. This may be one of the reasons why RT prices currently tend to be lower than DA prices in areas with large wind penetration. ${ }^{5}$ Wind

\footnotetext{
${ }^{5}$ As an example, within MISO's footprint in 2009, the average RT prices in areas with a substantial amount of wind energy (like North Dakota, Iowa, and Illinois) were as much as $5 \%$ lower than average DA prices. The difference between DA and RT prices was only $1-2 \%$ in the eastern part of the MISO footprint, which has less wind.
} 
power producers may therefore benefit from using wind power forecasts to bid into the DA market and thereby receive a higher and less volatile price. ${ }^{6}$

It is important to integrate the information in wind power forecasts into the DA market clearing. Wind power will have an increasing impact on the marginal cost of electricity, and this should be properly reflected in the DA market clearing, where most of the energy is settled. For the DA market to reflect the expected RT conditions as closely as possible, it is important that the market participants have the right incentives to provide the best possible wind power forecast information through their bids. Since the DA operation should be market-driven and based on the market participants' schedules and bids, the system operator should not use its own forecast to directly represent wind power generation in the SCUC/SCED procedure in the DA market. The system operators' forecast information should still be reflected in the operating reserve requirements, as discussed above, and in this way would also influence the market results.

One way to encourage participation of wind power generation in the DA market would be to reduce the time period between DA bidding and RT operations. The due times for DA bids vary among markets, but are at least 12 hours before the start of the operating day (Table 3-1). By shortening the time period and possibly introducing additional markets between DA and RT, the market participants, and particularly the wind power producers, could clearly make better use of forecast information, as the forecast error decreases with a shorter time horizon. It is also important to avoid situations in which market participants are incentivized to submit biased DA bids. As an example, the ERCOT market used to have protocols that unintentionally gave wind power producers in some areas incentives to over-schedule, due to allowances for uninstructed deviations combined with rules for congestion management [53]. In the Iberian electricity market (Spain and Portugal), asymmetric deviation penalties make it more attractive for wind power producers to bid above their expected generation [42].

\subsubsection{Reliability Assessment Commitment}

After the clearing of the DA market and before the start of the operating day, the ISO/RTO usually performs a revised commitment procedure, with more focus on reliability. The so-called reliability assessment commitment (RAC) is also performed using SCUC. However, at this stage the demand bids that were used to clear the DA market are replaced with the forecasted load for the next day. The ISO/RTO may therefore decide to change the commitment schedule from the DA market clearing based on the results from the RAC. The RAC procedure may take place according to a fixed time schedule or whenever the system operator finds it necessary to consider system re-commitment, due to unexpected operating conditions (forced outages, deviations from forecasted loads, etc.). Rules are typically in place to make sure that generating resources committed in the RAC recover all of their operating costs. This may sometimes require makewhole side payments, in addition to the regular payments based on the market clearing prices, in order to recover all costs (including no-load costs, start-up costs, etc.).

ISO/RTOs are currently focusing on how to integrate the information in wind power forecasts into the RAC. In contrast to the DA market clearing, the system operator can now use its own

\footnotetext{
${ }^{6}$ By using wind power forecasting for scheduling and bidding, wind power producers may also reduce their exposure to negative prices and lower their imbalance penalties (if such penalties exist). This is discussed in more detail in Chapter 7. In addition, WPF can play an important role in maintenance planning.
} 
forecast information directly as input to the SCUC. Hence, both demand and wind power bids/schedules are replaced with the system operators' own forecast information in order to ensure reliable operation. An important question is how to consider the additional uncertainty from wind power at this stage in the market operation. One can address the uncertainty and variability from wind power by adjusting and increasing the operating reserve requirements, as discussed above. However, the treatment of wind resources in the RAC will also influence how much other generation capacity is committed and available to accommodate the predicted and unpredicted system variability.

An interesting approach is taken by ERCOT, which is currently using an $80 \%$ exceedance forecast ${ }^{7}$ for wind power generation as input to its DA resource planning procedures [54]. ERCOT is considering whether to use the same type of forecast in the RAC as the nodal market system is introduced. In reality, using a conservative projection of wind power means that more capacity is committed than what would be the case if the mean or median forecast were used. Hence, while the resulting security margin may increase the reliability of the system, repeated over-commitment would result in inefficient operations.

An alternative way to address the uncertainty from wind power generation is to use a stochastic formulation of the UC problem. Several different approaches have been proposed in the literature recently to address uncertainty from wind power generation in the UC problem [55][56][57][58][59][60]. Preliminary results indicate that stochastic models can contribute to reducing costs while maintaining system security under increased uncertainty and variability. However, more research is needed into developing and testing stochastic models for UC and into evaluating their real-world applicability for system and market operations. In particular, it is important to generate consistent and representative information about the forecast uncertainty for use in such stochastic models. It is also important to consider the close interaction between the operating reserve requirements and the UC policy because they both influence the level of committed and available capacity. An important consideration is that while capacity committed as operating reserve receives reserve payments, additional capacity committed in the RAC is likely to receive make-whole side payments to recover costs, as pointed out above. The required level of capacity should be achieved in the most cost-efficient manner. Some of these issues are further explored in Chapters 4, 5, and 6 .

Another potential application of a wind power uncertainty forecast at this stage in the market operation is in congestion management analysis to assess the risk of congestion in some network branches and perform corrective measures to hedge against a higher risk of congestion [61].

\subsubsection{Real-time Operations}

Market participants can bid their remaining resources into the RT market. The deadline for submitting bids to the RT market varies quite widely between different markets (Table 3-1). ${ }^{8}$ During the operating hour, the ISO/RTO uses SCED to dispatch the system on the basis of the committed capacity in the system after the DA and RAC operations have been completed. At the same time, RT prices for energy (LMPs) and operating reserves are calculated. Conventional

\footnotetext{
${ }^{7}$ This means that there is an $80 \%$ chance that the actual wind power generation will exceed the forecast. Hence, it is equivalent to a $20 \%$ forecast quantile.

${ }^{8}$ Some ISO/RTOs, like NYISO and CAISO, also have a formal hour-ahead market clearing procedure.
} 
power generation sources are penalized if they deviate from their RT dispatch signals. Until recently, however, renewable generation such as wind power has not been given dispatch signals from the ISO/RTO and has typically been dispatched as a price taker in the RT market. Wind power generation also has exemptions from RT deviation penalties in most markets (Table 3-1).

ISO/RTOs are currently working on improving the integration of wind power into their RT dispatch procedures. Short-term wind power forecasts, which have relatively low uncertainty, should be taken into account in the ISO/RTOs' RT SCED. At the same time, it is important that the ISO/RTO is able to control the generation from wind power plants and enforce curtailment of wind power generation in situations where this is needed, either from an economic or reliability perspective. ${ }^{9}$ CAISO is requiring wind power plants in the Participating Intermittent Resource Program (PIRP) to bid into their hour-ahead market according to CAISO's short-term wind power forecast. Rules are also in place to limit the deviation penalties for wind power (Table 3-1).

NYISO recently introduced new rules to incorporate wind power in its SCED [62]. With the new rules, wind power plants will be required to bid into the RT market. A price-bid is offered into the market based on the wind power producer's preference, whereas the bid quantity is determined by the system operator's short-term wind power forecast. During unconstrained hours, wind power plants can operate freely. However, in constrained situations, wind power plants are directed to curtail their output when the clearing price at their location falls below their economic bid. Penalties are in place for exceeding the dispatch instructions. The new procedure makes certain that the economic preferences of the wind power producers are reflected in the SCED. It also contributes to a more efficient dispatch overall and reduces the need for using outof-market actions to maintain system reliability. PJM has also introduced a scheme for RT dispatch of wind power plants based on their bids. Other ISO/RTOs should consider similar measures to improve the handling of wind power resources in the RT dispatch.

\subsection{Summary and Conclusions}

WPF is an important tool to improve the efficiency and reliability of power systems with a large share of wind power. ISO/RTOs in the United States are currently working on integrating wind power forecasts into their operating procedures, and WPF is already used for a number of important applications. ${ }^{10}$ However, as the amount of wind power capacity is rapidly increasing, there is a need to better integrate WPF into different parts of electricity market operations, from DA scheduling to RT operations. It is also important to design electricity markets that give market participants the opportunity and right incentives to provide their unbiased forecast information through their scheduling and bidding.

Fig. 3-4 summarizes how we foresee that WPF will play important roles in the main steps in electricity market operations. ISO/RTOs and market participants will need to develop new tools and procedures to make efficient use of the forecast information, with the overall goal of making

\footnotetext{
${ }^{9}$ Modern wind power plants include a number of features that make them more similar to conventional power plants, including reactive power contribution, voltage regulation, disturbance ride-through, grid frequency response, smoothing wind ramps, and controlled startup/shutdown.

${ }^{10}$ More information on the status of the integration of wind power and forecasting in U.S. ISO/RTOs is provided by UWIG in [63][64].
} 
better operational decisions under the increased uncertainty and variability from wind power and other sources of renewable generation. A key challenge, therefore, is to improve decision making under uncertainty and the understanding of the impact of uncertainty on operational decisions (such as UC). At the same time, it is important to continue the improvements of WPF models, and to better tune them to the specific needs of the forecast users. For electricity market operators, it is particularly important to improve predictions of forecast uncertainty and ramping events.

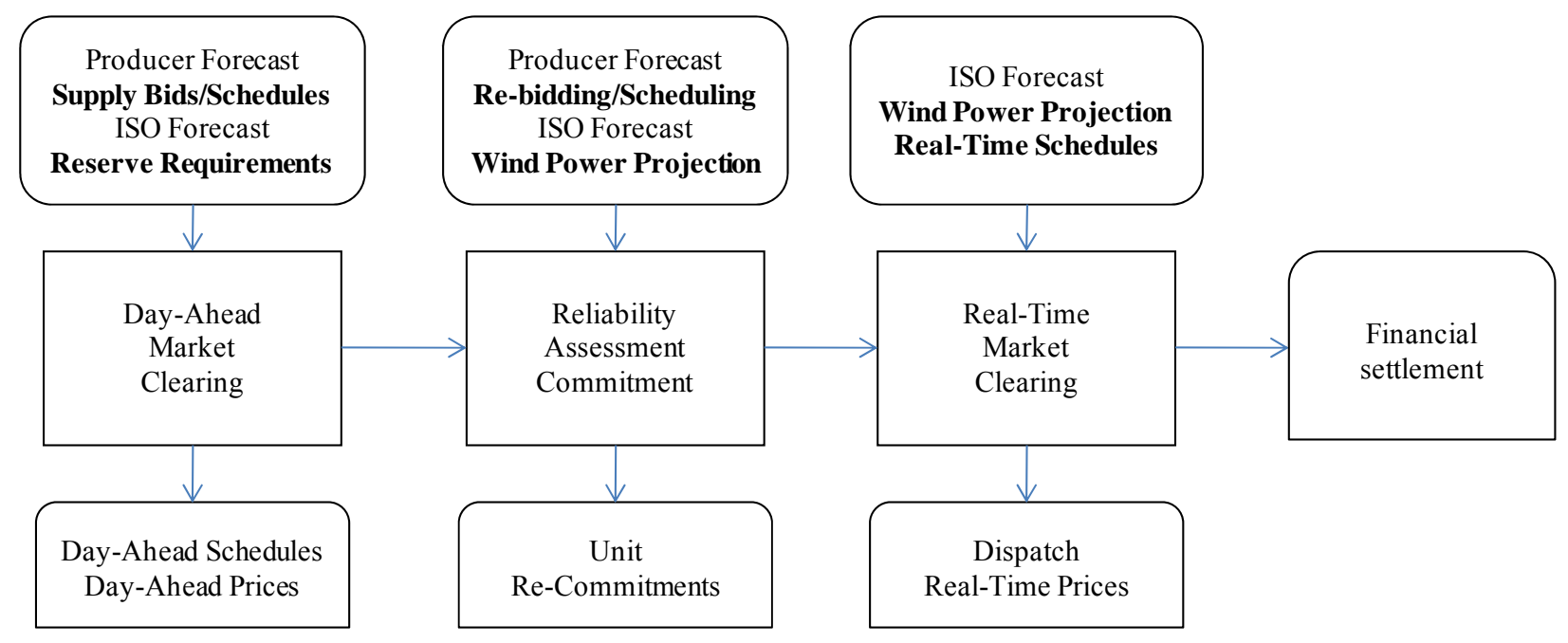

Fig. 3-4 Role of WPF in electricity market operations. 
This page intentionally blank. 


\section{WIND POWER FORECASTING UNCERTAINTY AND UNIT COMMITMENT ${ }^{11}$}

In this chapter, we investigate the representation of WPF uncertainty in the unit UC problem. While deterministic approaches use a point forecast of wind power output, WPF uncertainty in the stochastic UC alternative is captured by a number of scenarios that include cross-temporal dependency. A comparison among a diversity of UC strategies (based on a set of realistic experiments) is presented. The results indicate that representing WPF uncertainty with wind power scenarios that rely on stochastic UC has advantages over deterministic approaches that mimic the classical models. Moreover, the stochastic model provides a rational and adaptive way to provide adequate spinning reserves at every hour, as opposed to increasing reserves to predefined, fixed margins that cannot account either for the system's costs or its assumed risks.

The following nomenclature is used in this chapter:

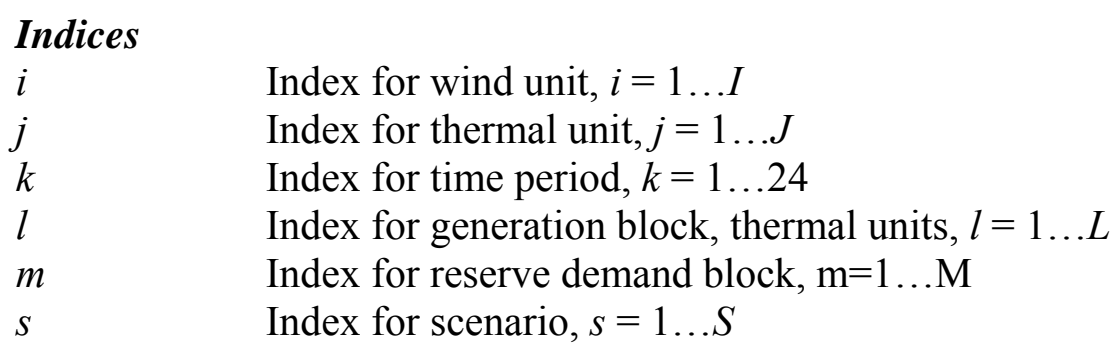

\section{Constants}

$\bar{\Delta}_{\mathrm{l}, \mathrm{j}}$

$\alpha(s)$

$a, b, c$

$A_{j}$

$C_{\text {ens }}$

$C C j$

$C R_{r n s, m}$

$D(k)$

$D C_{j}$

$H C j$

$M C_{l, j}$

$\operatorname{prob}_{s}$

$\overline{P T}_{j}$

$\mathrm{PT}_{j}$

$P W_{i}^{f, s}(k)$

$R L_{j}$

$S U_{j}$

$S D_{j}$

$T_{j}^{c o l d}$
Capacity, block $l$, thermal unit $j$

Operating reserve percentage, scenario $s$

Unit production cost function coefficients

Operating cost at minimum load, thermal unit $j$

Cost of energy not served

Cold start cost, thermal unit $j$

Cost of reserve not served, block $m$

Load or demand, period $k$

Shut-down cost, thermal unit $j$

Hot start cost, thermal unit $j$

Marginal cost (or bid), block $l$, thermal unit $j$

Probability of occurrence, wind scenario $s$

Capacity, thermal unit $j$

Minimum output, thermal unit $j$

Forecasted maximum generation, wind unit $i$, period $k$, scenario $s$

Ramping limit (up/down), thermal unit $j$

Start-up ramp limit, thermal unit $j$

Shut-down ramp limit, thermal unit $j$

Time for cold start cost (in addition to minimum downtime), thermal unit $j$

\footnotetext{
${ }^{11}$ This chapter is reproduced from Applied Energy, Vol. 88, No. 11, J. Wang, A. Botterud, R. Bessa, H. Keko, L. Carvalho, D. Issicaba, J. Sumaili, and V. Miranda, "Wind Power Forecasting Uncertainty and Unit Commitment,", pp. 4014-4023, 2011, with permission from Elsevier.
} 


\begin{tabular}{ll}
$T_{j}^{u p}$ & Minimum up-time, thermal unit $j$ \\
$T_{j}^{u p, 0}$ & Minimum up-time, initial time step, thermal unit $j$ \\
$T_{j}^{d n}$ & Minimum down-time, thermal unit $j$ \\
$T_{j}^{d n, 0}$ & Minimum down-time, initial time step, thermal unit $j$ \\
$W_{i}(k)$ & Actual maximum wind generation, wind unit $i$, period $k$ \\
$W R(k)$ & Additional wind reserve, period $k$ \\
& \\
$V a r i a b l e s$ & \\
$\delta_{l, j}(k)$ & Generation, block $l$, thermal unit $j$, period $k$ \\
$c_{j}^{p}(k)$ & Production cost, thermal unit $j$, period $k$ \\
$c_{j}^{u}(k)$ & Start-up cost, thermal unit $j$, period $k$ \\
$c w_{i}^{S}(k)$ & Curtailed wind generation, wind unit $i$, period $k$, scenario $s$ \\
$e n s$ & Energy not served, period $k$, scenario $s$ \\
$p t_{j}(k)$ & Generation, thermal unit $j$, period $k$ \\
\hline$p t_{j}(k)$ & Maximum feasible generation, thermal unit $j$, period $k$ \\
$p w_{i}^{s}(k)$ & Generation, wind unit $i$, period $k$, scenario $s$ \\
$r^{s}(k)$ & Reserve requirement (spinning), scenario $s$, period $k$ \\
$r n s_{m}^{s}(k)$ & Reserve curtailed, period $k$, scenario $s$ \\
$v_{j}(k)$ & Binary on/off variable, thermal unit $j$, period $k$
\end{tabular}

\subsection{Introduction}

UC and economic dispatch (ED) operate in tandem in the power generation industry to enable generation scheduling and grid management, thereby contributing to a system's overall reliability. However, the increasing use of renewable generation sources (such as wind power) is adding unprecedented amounts of uncertainty to a system operator's grid management and generation scheduling. Two key elements of this uncertainty - the inherent intermittency and the variability of renewable resources - are thus driving system operators to alter current industry approaches to UC and ED. Whereas considerable research effort has been directed to formulating and improving UC algorithms [65], efforts to consider wind power uncertainty have been more limited to date.

This chapter investigates the use of multiple wind power forecasts in UC and ED and analyzes the impact of different reserve requirements and UC policies on system operation. Our aim is to demonstrate that, despite the inherent uncertainties and risks when relying on wind power, it is possible for operators to adopt a quantified method to deal accurately with this renewable source. There are several reasons why UC and ED are greatly impacted when large amounts of wind energy are part of the mix of generation sources. First of all, WPF errors bring greater levels of uncertainty to system operation, because the RT amount of injected wind power may be very different from what is forecasted. Forecasting errors, which are mismatches between the value(s) plugged in during the UC stage versus the amounts used in the RT dispatch, can cause great difficulty for system operators, who must balance the unexpected surpluses or deficits of wind power. 
This forecasting uncertainty can be manifested in several ways. For example, an unforeseen reduction in wind power can hinder system reliability if the available ramping-up capabilities of the system's other on-line units are not sufficient to accommodate the current load. A large upward ramp in the supply of wind power may be unfavorable, however, in a system in which other units lack sufficient downward reserves. Wind power is sometimes curtailed to mitigate this situation, which nevertheless leads to an unwanted waste of available resources. In fact, a surplus in wind power at night may often have to be curtailed: while the wind may be strong, system load is usually low. In this case, wind generation may have to be spilled to maintain stable operation of thermal units (e.g., coal-fired, nuclear) because of their physical and/or economic constraints.

Variability is also a challenge for generation scheduling: because wind power is normally assumed to have an operating cost of zero, system operators try to make use of wind power as often as possible. To meet this objective, however, the output of other generation sources must be adjusted to accommodate wind power variability, which complicates scheduling the generation of non-wind resources. Therefore, even if wind power could be forecasted perfectly, variability by itself (i.e., considered in isolation) would still be important, because the system must still operate within the physical constraints of other non-wind units, such as their rampingup/-down constraints and minimum-on/minimum-off time constraints, etc.

These uncertainty factors complicate the challenges that operators and researchers face in revising and adjusting UC and ED algorithms to accommodate wind power, resulting in a variety of approaches. Some researchers focus on revising the current security-constrained unit commitment (SCUC) formulation. Others aim at novel UC methods. Barth et al. [55] presented the early stage of the Wind Power Integration in the Liberalised Electricity Markets (WILMAR) model in [66]. Tuohy et al. [59] extended their previous studies in [67] and [68] to examine the effects of stochastic wind and load on the UC and ED of power systems with high levels of wind power by using the WILMAR model. The WILMAR model calculates both UC decisions and the associated RT dispatch decisions using a rolling planning approach that uses realized wind power production and load in the first stage of the scenario trees. Ummels et al. [69] analyzed the impacts of wind power on thermal generation UC and ED in the Dutch system, which has a significant share of combined heat and power (CHP) units. Bouffard and Galiana [56] proposed a stochastic UC model to integrate significant wind power generation while maintaining the security of the system. Ruiz et al. [70] proposed a stochastic formulation to manage uncertainty in the UC problem. In a related paper [58], the authors consider uncertainty and variability in wind power in the UC problem by using the same stochastic framework. Wang et al. [57] presented an SCUC algorithm that takes the intermittency and variability of wind power generation into account. Delarue and D'haeseleer [71] develop a mixed-integer programming model to analyze the value of forecasting in unit commitment. Most of the models presented in the literature so far, however, are used for planning (i.e., UC) purposes (e.g., [55][57][58][66][70]), while there is only very limited research that explores the use of different WPF methods in actual market operation: in other words, the linkage between DA UC and RT ED is largely missing.

To deal with these issues in this chapter, we use (1) point and probabilistic forecasts of wind power that are generated by state-of-the-art techniques, and (2) two different methods: stochastic 
and deterministic. The consequences of a UC schedule to system costs are then assessed by running a dispatch simulation that takes into account the actual value of wind power instead of forecasted values. The relationship between UC policies and reserve requirements is discussed in the context of the large uncertainties arising from reliance on wind power. With added uncertainty, the focus shifts to considering some amount of extra reserve margin (e.g., see [72] and [73]). A detailed probabilistic analysis of the impact of renewable sources in the power system of the Iberian Peninsula is given in [74]. A methodology to define operating reserve considering conventional generation outages, load forecast uncertainty, and wind power forecast uncertainty is described in [75]. Probabilistic forecasts are used for wind power. In [76], a survey of the methods of determining the optimal amount of operating reserves for systems with high wind penetrations is presented, showing that various methods may have significantly different results. The challenge, therefore, is to define the amount of reserve needed for each hour adaptively and adequately instead of aiming at an arbitrarily fixed value for the required reserve. To contribute to this discussion, a number of cases are simulated in order to analyze and compare the results in detail.

In this study, a 24-hour optimization procedure is run at the beginning of the RT operation to represent the RT dispatch (assuming perfect knowledge of wind power in the following hours of the day). This way, the real cost of operation may be assessed fairly for comparison. Therefore, this constitutes a lower bound for the real cost of operation for a given commitment. In reality, there is still uncertainty in wind power for the subsequent time steps in relation to the current hour. This approach has the merit of taking into account the ramping constraints for the thermal units across the 24-hour operation horizon. In addition, because the objective is to compare UC models, we wanted to avoid blurring the analysis by simultaneously introducing a model for the ED that also included the uncertainty component. For similar reasons, we do not consider commitment decisions between DA scheduling and RT dispatch. In reality, the SO usually has some flexibility to commit additional fast-starting resources after the DA UC to account for any unexpected events, such as WPF deviations. This flexibility will contribute to improve the RT operation regardless of the DA UC strategy. However, in order to isolate the effect of the DA $\mathrm{UC}$, we do not consider the impact of intra-day commitments in this analysis.

The rest of the chapter is organized as follows: Section 4.2 briefly describes WPF and the scenario generation method we used. Section 4.3 presents the problem formulation. Numerical examples are provided in Section 4.4 Section 4.5 presents conclusions based on the discussion.

\subsection{Forecasting and Scenario Generation Approaches}

An advanced WPF system uses input data from different sources, and the forecast accuracy depends on a number of factors, including numerical weather prediction (NWP) models and the quality of wind predictions, local meteorological measurements, SCADA data (e.g., active power generated), and additional information (e.g., on the characteristics of the wind power plants, the complexity of the terrain, the availability of RT weather and power plant data). Large differences in forecasting errors can occur among wind power plants at different locations. WPF systems typically produce forecasts for a time horizon of up to 2-3 days ahead of the present time. In general, the forecasting error increases with the forecast horizon. We provide a detailed description of the state-of-the-art in WPF in [1]. 
There are several different approaches to model uncertainty in wind power predictions [1]. In this chapter, we use the approach from [77] to produce probabilistic forecasts based on point forecast errors. The model employs a linear quantile regression, where the base functions are formulated as cubic B-splines, in order to obtain the quantile with a proportion of the forecast errors. The probabilistic forecast is represented through a set of quantiles ranging from $5 \%$ to 95\% (in 5\% increments), and the tails are modeled by exponential interpolation reflecting improbable and extreme events [77].

Since the probabilistic forecasts (i.e., set of quantiles) produced by the quantile regression method do not capture the temporal correlation between forecast errors (i.e., uncertainty) of different hours, a scenario generation method described in [78] is used to generate a number of wind power scenarios that provide information on the development of the prediction errors through the set of look-ahead times. The method takes as inputs the forecasted quantiles for each look-ahead time-step and also the observed wind power generation. Assuming that the probabilistic forecasts are reliable, the forecast errors are made Gaussian by applying a transformation with the inverse of the Gaussian cumulative distribution function. This results in a Gaussian random variable with zero mean and unit standard deviation. Considering the vector with all the forecasts for each look-ahead time-step, it is assumed that the random vector follows a multivariate Gaussian distribution, with mean values being a vector of zeros and a covariance matrix. The temporal interdependence structure is represented by the covariance matrix, which is recursively estimated because of the nonstationary characteristics. A specified number of scenarios are obtained through sampling from an inverse cumulative distribution function. More details about the method can be found in [78]. The scenarios produced by the method have the following characteristics: (i) they respect the marginal wind power forecasted distribution for the coming horizon (i.e., probabilistic forecasts); and (ii) the temporal correlation of forecast errors are embedded in the scenarios values.

\subsection{Unit Commitment and Dispatch Formulations}

The UC and dispatch formulations used in this chapter expand on our initial version of the UC problem introduced in [60]. The general UC constraints follow the deterministic model in [79]. However, we make adjustments in the stochastic version on the basis of introducing WPF uncertainty, which is represented in the form of scenarios.

\subsubsection{Objective Function}

The objective is to minimize the sum of three kinds of costs: expected production costs, the expected cost of unserved energy and reserve curtailment, and start-up costs, as shown in (4-1). Constraints on load and operating reserves are represented in (4-2)-(4-3). We use a step-wise reserve demand curve to mimic the reserve requirement practiced by some system operators, such as MISO [80], as shown in (4-4). This formulation allows the reserve requirement, which is represented by a certain percentage of load, to be reduced in some cases at the reserve curtailment cost captured in (4-1) to avoid load curtailment. We also introduce the possibility of dedicating an additional operating reserve at the DA UC stage specifically for use with wind power generation. The idea is that this wind power reserve helps accommodate the uncertainty and variability that arise from reliance on wind units. Wind units may also be curtailed, if necessary, as shown in (4-5). Note that the dispatch of thermal units - and therefore the costs associated with production, curtailed energy, and reserve - all vary by wind scenario. 
Furthermore, the constraints for load, operating reserves, and wind curtailment must be met in all wind scenarios. In contrast, thermal units' start-up costs are independent of the various wind scenarios because we assume that the commitment of thermal units has to be fixed at the DA stage.

We also assume that each thermal unit is offered into the market as a step-wise, price-quantity offer function and that the offers can be derived by linearizing a standard quadratic production cost function. Thus, we can express the operating cost for one thermal unit with the equations in (4-6)-(4-9). The coefficients for the generation blocks are derived from the quadratic production cost function. The last part of the objective function is the start-up cost, which is modeled by assuming that there is a cold start-up cost and a warm start-up cost, depending on the length of time that a given unit has been ramped down. The mathematical formulation is shown in (4-10)(4-12).

\subsubsection{Thermal Unit Constraints}

The constraints for the operation of thermal units include generation limits, ramping-up and -down limits, and minimum-up and minimum-down time. The upper and lower generation limits for the thermal plants are shown in (4-13). The maximum power output of a unit, $\overline{p t_{J}^{s}}(k)$, is constrained by the generation limit of a unit in (4-14), limitations on start-up and ramp-up rates in (4-15), shut-down ramp rates in (4-16), and ramp-down limits in (4-17). The availability of spinning reserves is equal to the difference between the maximum potential generation and the actual generation, that is, $\overline{p t_{j}^{s}}(k)-p t_{j}^{S}$. Thus, the reserve requirement in (4-3) takes into account the constraints imposed by (4-13)-(4-17). The reserve requirement is maintained for each individual wind scenario.

The final constraints included are the minimum-up and -down time constraints. Minimum-up times are represented by (4-18)-(4-20), which represent the initial status, the intermediate time periods, and the final time steps of the planning period, respectively. The minimum-down time constraints are represented analogously by (4-21)-(4-23). Note that the equations for generation and ramping limits, (4-13)-(4-17), must be included for all wind scenarios, because thermal dispatch depends on the wind generation. In contrast, the minimum-up and -down time constraints, (4-18)-(4-23), are functions of commitment only and do not vary with wind scenarios. Note that the calculation of the $T_{j}^{u p, 0}$ variable depends on the initial state and minimumon time constraints of the unit. If the unit has been on for less than the minimum on time, the unit has to remain on until it meets the minimum on time constraint. A similar logic applies to $T_{j}^{d n, 0}$ as well. For simplicity's sake, transmission constraints are currently not represented in the model, although their absence does not change the conclusions qualitatively. 


$$
\begin{aligned}
\operatorname{Min} \sum_{s=1}^{S} \operatorname{prob}_{s} & \cdot\left\{\sum_{k=1}^{K} \sum_{j=1}^{J} c_{j}^{p, s}(k)+\sum_{k=1}^{K} C_{e n s} \times e n s^{s}(k)+\sum_{k=1}^{K} \sum_{m=1}^{M} C R_{r n s, m} \times r n s_{m}^{s}(k)\right\} \\
& +\sum_{k=1}^{K} \sum_{j=1}^{J} c_{j}^{u}(k)
\end{aligned}
$$

s.t.

$\sum_{i=1}^{I} p w_{i}^{s}(k)+\sum_{j=1}^{J} p t_{j}^{s}(k)=D(k)-e n s^{s}(k), \quad \forall k, \forall s$

$\sum_{j=1}^{J}\left[\overline{p t}{ }_{j}^{s}(k)-p t_{j}^{s}(k)\right] \geq r^{s}(k), \quad \forall k, \forall s$

$r^{s}(k)=D(k) * \alpha(s)-\sum_{m=1}^{M} r n s_{m}^{s}(k)+W R(k), \quad \forall k, \forall s$

$p w_{i}^{s}(k)+c w_{i}^{s}(k)=P W_{i}^{f, s}(k), \forall i, \forall k, \forall s$

$c_{j}^{p, s}(k)=A_{j} v_{j}(k)+\sum_{l=1}^{L} M C_{l, j}(k) \cdot \delta_{l, j}^{s}(k), \quad \forall j, \forall k, \forall s$

$p t_{j}^{S}(k)=\underline{P T_{j}} \cdot v_{j}(k)+\sum_{l=1}^{L} \delta_{l, j}^{s}(k), \quad \forall j, \forall k, \forall s$

$\delta_{l, j}^{s}(k) \leq \bar{\Delta}_{\mathrm{l}, \mathrm{j}} \quad, \quad \forall l, \forall j, \forall k, \forall s$

$\delta_{l, j}^{s}(k) \geq 0 \quad, \quad \forall l, \forall j, \forall k, \forall s$

$c_{j}^{u}(k) \geq C C_{j} \cdot\left[v_{j}(k)-\sum_{n=1}^{N} v_{j}(k-n)\right], \forall j, \forall k$

where $N=T_{j}^{d n}+T_{j}^{\text {cold }}$

$c_{j}^{u}(k) \geq H C_{j} \cdot\left[v_{j}(k)-v_{j}(k-1)\right], \quad \forall j, \forall k$

$c_{j}^{u}(k) \geq 0, \quad \forall j, \forall k$

$\underline{P T_{j}} \cdot v_{j}(k) \leq p t_{j}^{S}(k) \leq \overline{p t_{j}^{S}}(k) \quad, \quad \forall j, \forall k, \forall s$

$0 \leq \overline{p t}_{j}^{s}(k) \leq \overline{P T}_{j} \cdot v_{j}(k) \quad, \quad \forall j, \forall k, \forall s$

$\overline{p t_{j}^{S}}(k) \leq p t_{j}^{S}(k-1)+R L_{j} \cdot v_{j}(k-1)+S U_{j} \cdot\left[v_{j}(k)-v_{j}(k-1)\right]+$ $\overline{P T}_{j} \cdot\left[1-v_{j}(k)\right] \quad, \quad \forall j, \forall k, \forall s$

$\overline{p t_{j}^{s}}(k) \leq \overline{P T}_{j} \cdot v_{j}(k+1)+S D_{j} \cdot\left[v_{j}(k)-v_{j}(k+1)\right], \quad \forall j, \forall k=1 . .23, \forall s$ 


$$
\begin{aligned}
& p t_{j}^{S}(k-1)-p t_{j}^{S}(k) \leq R L_{j} \cdot v_{j}(k)+S D_{j} \cdot\left[v_{j}(k-1)-v_{j}(k)\right]+ \\
& \overline{P T}_{j} \cdot\left[1-v_{j}(k-1)\right], \forall j, \forall k, \forall s \\
& \sum_{k=1}^{T_{j}^{u p, 0}}\left[1-v_{j}(k)\right]=0, \quad \forall j \\
& \sum_{n=k}^{k+T_{j}^{u p}-1} v_{j}(n) \geq T_{j}^{u p} \cdot\left[v_{j}(k)-v_{j}(k-1)\right], \quad \forall j, \forall k=T_{j}^{u p, 0}+1, \ldots, T-T_{j}^{u p}+1 \\
& \sum_{n=k}^{T}\left\{v_{j}(n)-\left[v_{j}(k)-v_{j}(k-1)\right]\right\} \geq 0, \forall j, \forall k=T-T_{j}^{u p}+2, \ldots, T \\
& \sum_{k=1}^{T_{j}^{d n, 0}} v_{j}(k)=0 \quad, \quad \forall j \\
& \sum_{n=k}^{k+T_{j}^{d n}-1}\left[1-v_{j}(n)\right] \geq T_{j}^{d n} \cdot\left[v_{j}(k-1)-v_{j}(k)\right] \\
& \forall j, \forall k=T_{j}^{d n, 0}+1, \ldots, T-T_{j}^{d n}+1 \\
& \sum_{n=k}^{T}\left\{1-v_{j}(n)-\left[v_{j}(k-1)-v_{j}(k)\right]\right\} \geq 0, \quad \forall j, \forall k=T-T_{j}^{d n}+2, \ldots, T
\end{aligned}
$$

\subsubsection{Deterministic Formulation}

In a simplified representation, the formulation above would consider only one scenario for the forecasted wind generation. In such a case, the formulation would be equivalent to a deterministic version of the UC problem. The selected scenario could be the expected wind power generation or point forecast or could also represent a certain quantile in the forecasting probability distribution. This formulation is useful for comparison purposes.

\subsubsection{Economic Dispatch}

In order to assess the dispatch cost in RT, we also develop an ED formulation. The commitment variables are now assumed to be fixed from the UC run. The representation of wind power generation by scenarios is replaced by the realized wind power output (without considering potential wind power curtailment). Hence, we formulate a deterministic ED problem consisting of equations (4-1)-(4-9) and (4-13)-(4-17), with only one wind power scenario and fixed values for the thermal commitment variables, $v_{j}(k)$. The start-up cost and minimum-up and -down time constraints are not considered because of the fixed commitment. Ramping constraints are in (4-13)-(4-17), and the 24-hour problem is solved in one shot. Notably, the operating reserve requirement in (4-3) is also imposed in the ED formulation. As discussed in the introduction, we assume that the dispatch is carried out with perfect knowledge of the actual wind power being injected into the system for future time steps. 
A market simulation has been set up that first solves the UC by making use of the wind power forecast, and then solves the ED by using the realized wind conditions. This action is performed in sequence for multiple days. An updated wind power forecast, along with the unit status and generation output for the thermal units from the previous day, are taken as initial conditions for the UC problem for the next day. The main results (UC, dispatch, available reserves, unserved load, curtailed reserve, prices, etc.) are calculated and stored after each simulation day.

Because the focus is on quantifying the impact of wind power forecasts on system operation, the only uncertainty we consider derives from wind generation. Other uncertainties, such as load or forced outage, are not considered directly. We assume that operating reserves maintained in the RT dispatch and based on classical criteria are adequate to accommodate these uncertainties. As a result, the additional amount of "wind power reserve" becomes critical to addressing the impact of uncertainty when using wind power. With the stochastic UC formulation, the need for additional operating reserves is arguably already addressed because we include a representative set of wind power outcomes in the scenarios. However, even if the scenarios are highly accurate in capturing all of the possible wind power outputs, additional reserve may be needed even in the stochastic formulation to cover any extra uncertainty that the simulated scenarios are not able to capture. In the case study below, we run a number of different cases to investigate the impact of UC strategy and operating reserve policy on the system dispatch.

\subsection{Case Study}

\subsubsection{Assumptions}

To provide a didactic and clear case study, we use a test power system to simulate the impact of using different wind power forecasts and operating reserve policies. The UC and ED are run in sequence as described above. The main assumptions for the case study are outlined below.

The characteristics of the thermal power plants are based on the case studies presented in [79] and [81], although we have modified some of the cost coefficients and also introduced data for ramping rates. The resulting input parameters are shown in Table 4-1. Each unit is represented with four blocks of equal size. The bid price of each block is calculated by linearizing the quadratic cost function. The production cost increases from baseload units to peak units. The hourly profile of the loads is taken from the historical data for two utilities in the state of Illinois for the three months of October through December of 2006. The overall load level is scaled down to match the generation capacity in the test power system.

The operating reserve (spinning) requirement in the UC formulation is assumed to be $10 \%$ of the system load as the default value. The required operating reserves have to be met by the thermal power plants (i.e., wind power plants cannot provide reserves). Note that the stochastic model allows the use of different reserve requirements in each scenario. In the case study, however, we use the same value in all scenarios. We investigate the consequences of increasing the reserve requirement in the $\mathrm{UC}$, as discussed above, whereas the reserve requirement is kept constant at $10 \%$ of load in the ED problem, representing RT operations. We assume only one step in the spinning reserve demand curve. The cost of reserve curtailment is $\$ 1,100 / \mathrm{MWh}$, and the cost of unserved energy is $\$ 3,500 / \mathrm{MWh}$, which corresponds to the current practice at the MISO in the 
United States [82]. Thus, reserves will be curtailed ahead of load. It should be noted that the computational results are influenced by these penalty prices for unserved load and reserve. ${ }^{12}$

Table 4-1 Generator data.

\begin{tabular}{|c|c|c|c|c|c|c|}
\hline Unit & $\begin{array}{c}\overline{P T}_{j} \\
{[\mathrm{MW}]}\end{array}$ & $\frac{P T_{j}}{[\mathrm{MW}]}$ & $\begin{array}{c}R L_{j}^{\mathrm{a}} \\
{[\mathrm{MW} / \mathrm{h}]}\end{array}$ & $\begin{array}{c}T_{j}^{u p} \\
{[\mathrm{~h}]}\end{array}$ & $\begin{array}{c}T_{j}^{d n} \\
{[\mathrm{~h}]}\end{array}$ & $\begin{array}{c}\text { In. } \\
\text { state } \\
{[\mathrm{h}]}\end{array}$ \\
\hline Base1 & 455 & 150 & 200 & 8 & 8 & 8 \\
\hline Base2 & 455 & 150 & 200 & 8 & 8 & 8 \\
\hline Int1 & 130 & 20 & 100 & 5 & 5 & -5 \\
\hline Int2 & 130 & 20 & 100 & 5 & 5 & -5 \\
\hline Int3 & 162 & 25 & 100 & 6 & 6 & -6 \\
\hline Int4 & 80 & 20 & 80 & 3 & 3 & -3 \\
\hline Int5 & 85 & 25 & 85 & 3 & 3 & -3 \\
\hline Peak1 & 55 & 10 & 55 & 1 & 1 & -1 \\
\hline Peak2 & 55 & 10 & 55 & 1 & 1 & -1 \\
\hline Peak3 & 55 & 10 & 55 & 1 & 1 & -1 \\
\hline Unit & $\begin{array}{c}a_{j} \\
{[\$ / \mathrm{h}]}\end{array}$ & $\begin{array}{c}b_{j} \\
{[\$ / \mathrm{MWh}]}\end{array}$ & $\begin{array}{c}c_{j} \\
{\left[\$ / \mathrm{MW}^{2} \mathrm{~h}\right]}\end{array}$ & $\begin{array}{c}C C_{j} \\
{[\$ / \mathrm{h}]}\end{array}$ & $\begin{array}{l}H C_{j} \\
{[\$ / \mathrm{h}]}\end{array}$ & $\begin{array}{c}T_{j}^{\text {cold }} \\
{[\mathrm{h}]}\end{array}$ \\
\hline Base1 & 1,000 & 16 & 0.00048 & 9,000 & 4,500 & 5 \\
\hline Base2 & 970 & 17 & 0.00031 & 10,000 & 5,000 & 5 \\
\hline Int1 & 700 & 30 & 0.002 & 1,100 & 550 & 4 \\
\hline Int2 & 680 & 31 & 0.0021 & 1,120 & 560 & 4 \\
\hline Int3 & 450 & 32 & 0.004 & 1,800 & 900 & 4 \\
\hline Int4 & 370 & 40 & 0.0071 & 340 & 170 & 2 \\
\hline Int5 & 480 & 42 & 0.00079 & 520 & 260 & 2 \\
\hline Peak1 & 660 & 60 & 0.0041 & 60 & 30 & 0 \\
\hline Peak2 & 665 & 65 & 0.0022 & 60 & 30 & 0 \\
\hline Peak3 & 670 & 70 & 0.0017 & 60 & 30 & 0 \\
\hline
\end{tabular}

\subsubsection{Wind Power and Forecast Characteristics}

We used time series of the DA deterministic point forecasts and realized wind power output for 15 hypothetical locations in the state of Illinois in 2006 as our wind power data, which were obtained from the Eastern Wind Integration and Transmission Study [83]. The data were produced by combining a mesoscale weather model with a composite power curve for a number of potential sites for wind power farms. The DA forecasts were generated based on observed forecast errors from four real wind power plants. The data methodology is explained in [84]. Data for the 15 sites were aggregated into one time series. The accuracy of the DA wind power forecast varies from day to day. For the point forecast, the normalized mean average errors (NMAEs) over a 91-day simulation period vary between $8.4 \%$ and $12.4 \%$ for different hours of the day, with the highest forecast errors occurring in the afternoon between noon and 6:00 p.m.

The total installed capacity of wind power is assumed to be $500 \mathrm{MW}$, and for simplicity we model this amount as one large wind power plant. For the simulated period, the wind power capacity factor is $39.9 \%$, and the wind power meets $20.2 \%$ of the load (with no wind

\footnotetext{
${ }^{12}$ We have run sensitivity case studies on the penalty prices. The results show that, whereas the commitment and dispatch of thermal units do not change much, the costs of reserve and load curtailment do naturally depend on these values.
} 
curtailment). Wind power and load are uncorrelated with a correlation coefficient of 0.01 . With these assumptions, the total installed capacity of the thermal units is $10.8 \%$ higher than the peak load. If we assign a capacity value of $20 \%$ to the wind power capacity, the system reserve margin increases to $17.4 \%$.

To generate the wind power scenarios, we used the wind power data (forecasts and realized generation) for the period from January to July to train the quantile regression, and then the months from August to December are used as a test dataset. Because the first month is used only to initialize the estimation of the covariance matrix, the scenarios are only produced for October, November, and December.

\subsubsection{Simulated Cases}

To compare the results of using different wind power forecasts and UC formulations (deterministic vs. stochastic), and also the different UC policy and reserve requirements, we have conducted tests of a list of 10 simulation cases as summarized in Table 4-2. The cases are in three subgroups: forecasts, reserve requirements, and stochastic UC. In the forecasts subgroup, the first case (F1) is a reference case with a perfect wind power forecast. Case F2 uses the wind power point forecast. In case F3, the UC is performed without considering the use of wind power at all. In F4 and F5, different forecasts in terms of different quantiles are used. F4 uses the 20\% quantile forecast instead of the point forecast, which is similar to the current practice for DA scheduling in ERCOT [85]. F5 uses the 50\% quantile forecast and aims to investigate the performance of the median forecast.

Table 4-2 Simulated cases.

\begin{tabular}{|c|c|c|c|}
\hline Case & Description & $U C$ & Forecast \\
\hline \multicolumn{4}{|c|}{ Subgroup 1-Forecasts } \\
\hline F1 & Deterministic (Det.) UC w/perfect forecast & Det. & Perfect \\
\hline F2 & Det. UC w/point forecast & Det. & Point \\
\hline F3 & Det. UC w/no forecast & Det. & No \\
\hline $\mathrm{F} 4$ & Det. UC w/20\% quantile forecast & Det. & Point \\
\hline F5 & Det. UC w/50\% quantile forecast & Det. & Point \\
\hline \multicolumn{4}{|c|}{ Subgroup 2 - Reserve Requirements } \\
\hline R1 & $\begin{array}{l}\text { Det. UC w/additional reserve } \\
\text { ( } 20 \% \text { of point forecast) }\end{array}$ & Det. & Point \\
\hline R2 & $\begin{array}{l}\text { Det. UC w/additional reserve } \\
\text { (point forecast }-10 \% \text { quantile forecast) }\end{array}$ & Det. & Point \\
\hline R3 & $\begin{array}{l}\text { Det. UC w/additional reserve } \\
\text { (additional } 5 \% \text { of load) }\end{array}$ & Det. & Point \\
\hline \multicolumn{4}{|c|}{ Subgroup 3 - Stochastic UC } \\
\hline S1 & Stochastic UC w/regular reserve & Stoch. & Scenarios \\
\hline $\mathrm{S} 2$ & Stochastic UC w/additional reserve (additional $5 \%$ of load) & Stoch. & Scenarios \\
\hline
\end{tabular}

The reserve requirements subgroup illustrates the impact of different reserve requirements on the deterministic UC results. We assume that a basic reserve requirement of $10 \%$ of the load is adequate to accommodate load forecast errors and forced outages of generating units.

In R1, an additional $20 \%$ of the point forecast is added to the reserve requirement to accommodate wind power uncertainty. In other words, the wind reserve, $W R(k)$, in Eq. (4-4) is equal to $20 \%$ of the point forecast. In R2, we calculate the additional wind reserve requirement 
by deducting the $10 \%$ quantile forecast from the point forecast. Because the realized wind power will most likely be higher than the $10 \%$ quantile forecast ( $90 \%$ of the time), R2 should be able to accommodate the uncertainty in WPF to a large extent. In R3, we increase the level of reserves from $10 \%$ to $15 \%$ of the load, so that in this case, the additional wind reserve does not consider the information in the wind power forecast.

Finally, case S1 uses stochastic UC with a regular reserve requirement, and case 2 is the stochastic UC with the additional reserve (15\% of the system load instead of $10 \%$ in S1). The stochastic UC cases use a total of 10 wind power scenarios, which not always be sufficient to capture the full range of potential outcomes for the wind power generation for the next day. The purpose of increasing the reserve in $\mathrm{S} 2$ is to use the additional reserve to cover the extra uncertainty in wind power not captured by the scenarios.

\subsubsection{Results}

In this section, we provide detailed dispatch results for one selected day and the overall simulation results to show the short-term impact of different system scheduling methods and longer-term simulation statistics with different wind power forecasts.

\subsubsection{Results for One Day}

We present the dispatch results for day 87 , which is selected because there is a relatively large forecasting error on this day. Fig. 4-1 shows that both the point forecast and most of the scenarios predict too much wind power for most of the day. Fig. 4-2 shows the number of units on-line in cases F2, R3, and S1, which are the most likely alternatives to represent how system operators may incorporate wind power forecasts into their UC. In this figure, we can see the number of on-line units in S1 is higher than in F2 and higher in R3 than in S1.

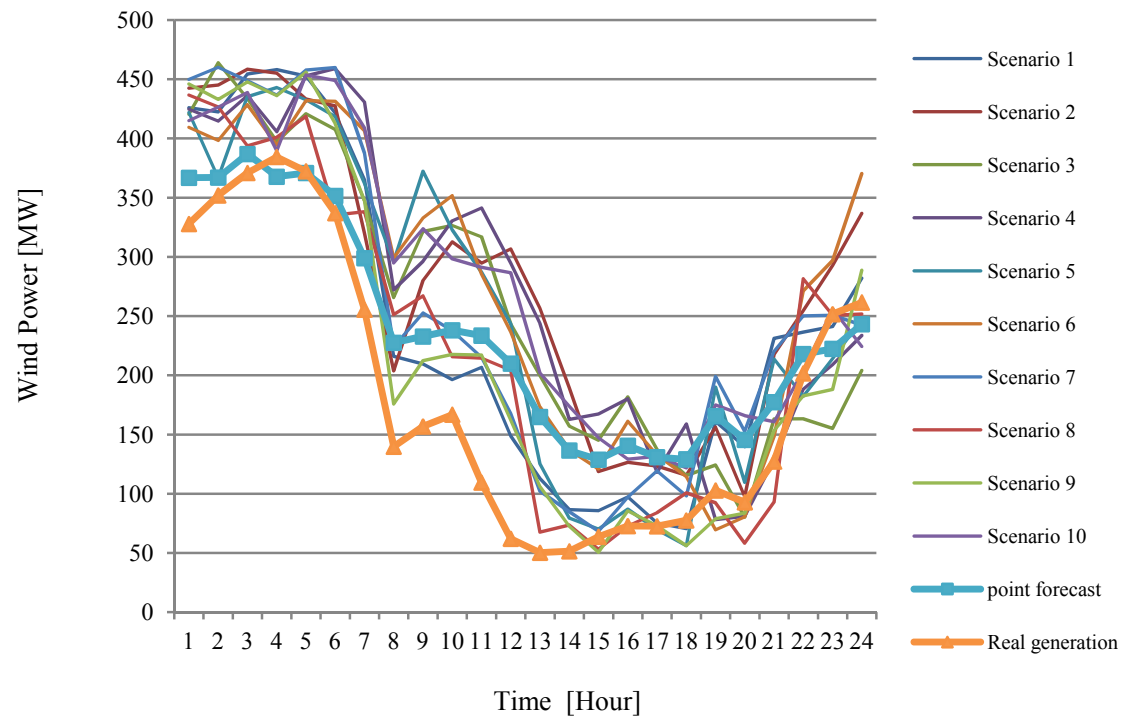

Fig. 4-1 Wind power forecast (deterministic point forecast and 10 stochastic scenarios) and realized wind generation for day 87. 
This result is not surprising, given that more generating units should be expected to be scheduled on-line in S1 compared to F1, to provide sufficient ramping capability to handle the different wind power scenarios (Fig. 4-1). A parallel effect is obtained with R3 by imposing a higher reserve requirement at the UC stage. Fig. 4-3 shows the level of available operating reserves in the RT dispatch for the three alternatives. The available capacity surplus is derived by subtracting the dispatch, $p t_{j}(k)$, from the sum of maximum feasible generation, $\overline{p t}_{j}(k)$, for each thermal unit and adding up the results. It is of note that in F2, the available capacity drops below the required ED reserve requirement for several hours. This is particularly the case in hours 11 and 12 when there is a large deviation between the realized wind generation and the point forecast. This deviation causes the use of all of the available capacity, including operating reserves, and eventually leads to the load curtailment in those two hours (Fig. 4-3) for F2.

In this case, it is evident that the stochastic strategy S1 provided a better overall solution. The available operating reserves from S1 match well with the ED reserve requirement in Fig. 4-3 with only minor shortages in hours 8 and 9 , while R3 leads to an excessive amount of available reserves as shown between hours 8 and 22 in Fig. 4-3.

\subsubsection{Results for a Three-month Period}

Fig. 4-4 shows the total hours of commitment for the ten thermal units in a three-month simulation period. Total hours of commitment are the highest in F3, which does not take wind power into account in the UC and therefore needs more units to be committed to compensate for the absence of the wind power generation (available in all of the other cases). This case illustrates that if system operators are not using the information in the wind power forecast, the over-commitment of thermal units may easily result. The higher reserve requirement in S2, plus the wind uncertainty (represented by the scenarios), led to a high commitment of units in S2, as well. Case F2 has the lowest commitment of units, which leads to lower available capacity for reserve and higher curtailment, as shown next.

Table 4-3 shows the average dispatch of thermal units for all of the cases. The results show that the differences in dispatch are relatively smaller than are the differences in commitment. This result occurs because the same realized wind generation output is used in the RT dispatch in all of the cases. The resulting average thermal dispatch therefore becomes similar in the ten cases, despite the differences in commitment. For the peaking plants, note that they are being dispatched at their minimum levels most of the time while committed. 


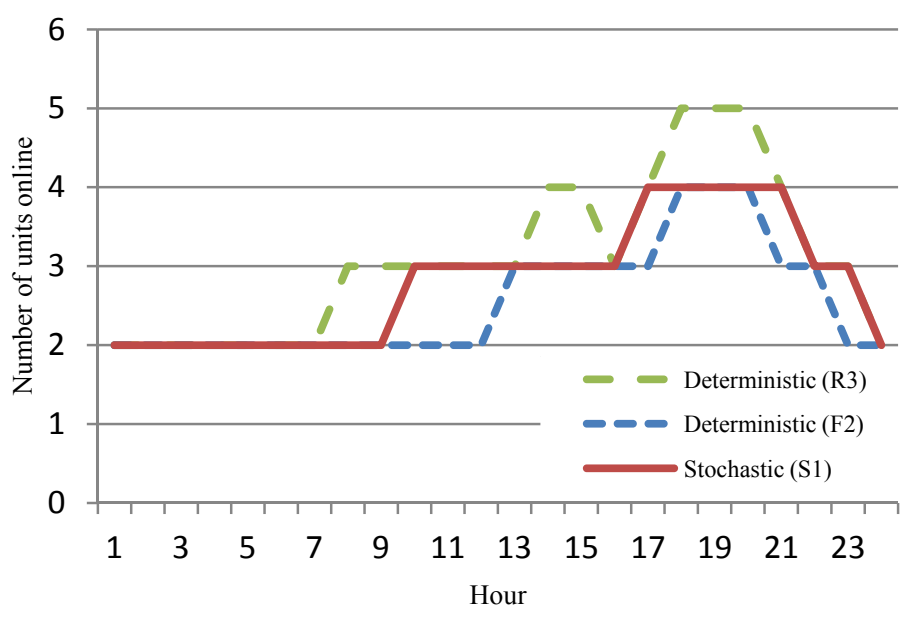

Fig. 4-2 Number of on-line units for day 87.

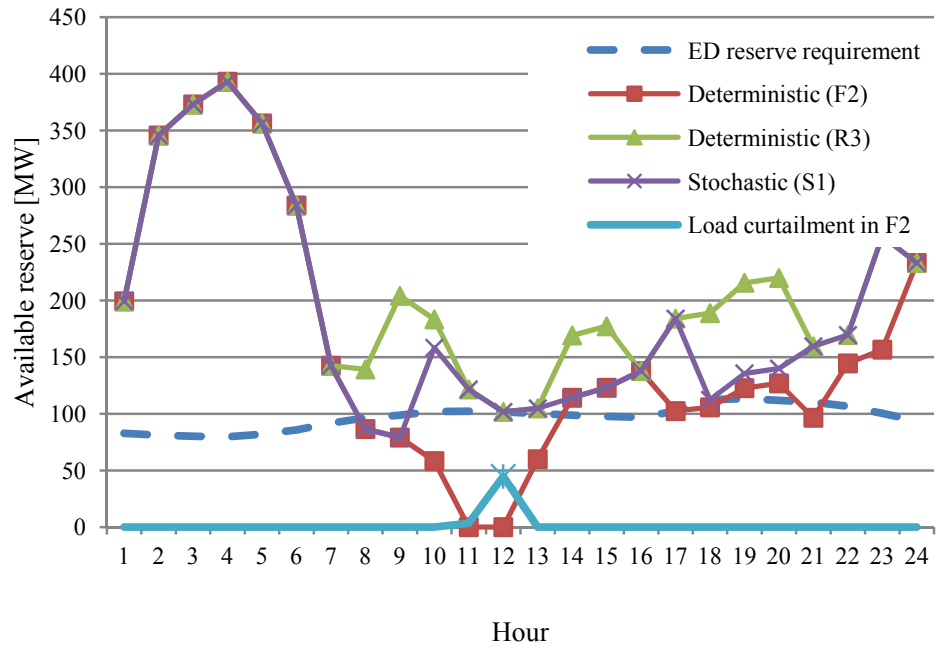

Fig. 4-3 Available operating reserves in RT dispatch.

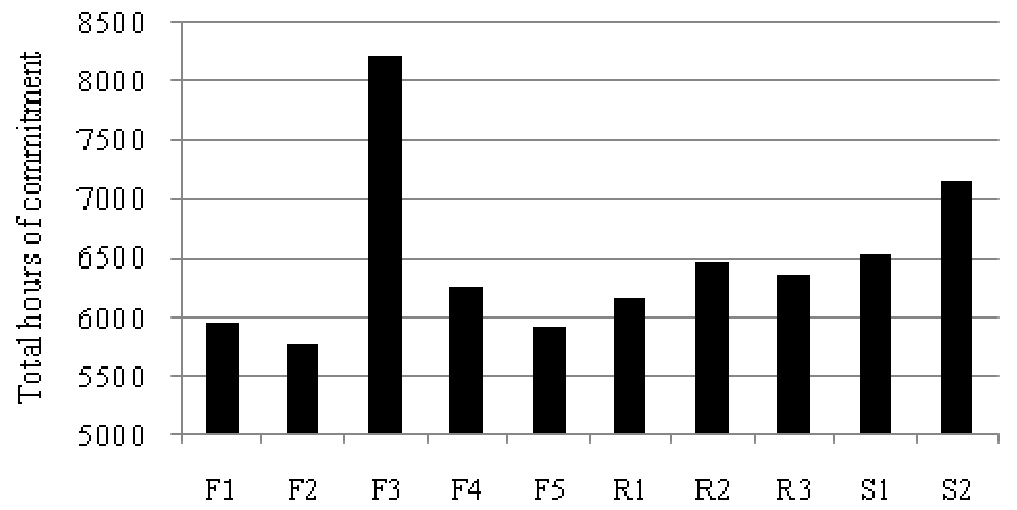

Fig. 4-4 Total hours of commitment for thermal units in all cases. 
Table 4-3 Average dispatch for thermal units [MW].

\begin{tabular}{|c|c|c|c|c|c|}
\hline Unit & $\begin{array}{l}\text { F1 (Perfect } \\
\text { forecast) }\end{array}$ & $\begin{array}{l}\text { F2 (Point } \\
\text { forecast) }\end{array}$ & $\begin{array}{l}\text { F3 (No } \\
\text { forecast) }\end{array}$ & $\begin{array}{c}\mathrm{F} 4(20 \% \\
\text { quantile } \\
\text { forecast) }\end{array}$ & $\begin{array}{c}\mathrm{F} 5(50 \% \\
\text { quantile } \\
\text { forecast) }\end{array}$ \\
\hline Base1 & 441.3 & 440.5 & 436.0 & 440.8 & 440.8 \\
\hline Base2 & 315.4 & 316.6 & 300.9 & 313.6 & 315.9 \\
\hline Int1 & 13.9 & 8.4 & 22.4 & 15.6 & 11.2 \\
\hline Int2 & 1.9 & 1.2 & 3.7 & 1.8 & 1.2 \\
\hline Int3 & 15.3 & 21.3 & 23.0 & 16.2 & 18.2 \\
\hline Int4 & 2.3 & 1.9 & 3.5 & 1.9 & 2.5 \\
\hline Int5 & 0.1 & 0.1 & 0.5 & 0.2 & 0.2 \\
\hline Peak1 & 0.2 & 0.2 & 0.3 & 0.2 & 0.3 \\
\hline Peak2 & 0.0 & 0.0 & 0.1 & 0.0 & 0.0 \\
\hline Peak3 & 0.0 & 0.0 & 0.0 & 0.0 & 0.0 \\
\hline Unit & $\begin{array}{l}\mathrm{R} 1 \text { (wind pf } \\
\text { resrve) }\end{array}$ & $\begin{array}{l}\text { R2 (wind } \\
\text { quantile } \\
\text { reserve) }\end{array}$ & $\begin{array}{l}\text { R3 (load } \\
\text { reserve) }\end{array}$ & $\begin{array}{c}\text { S1 (Stochastic } \\
\text { UC) }\end{array}$ & $\begin{array}{l}\text { S2 (Stochastic } \\
\quad+\text { reserve) }\end{array}$ \\
\hline Base1 & 440.5 & 440.5 & 440.5 & 440.5 & 440.3 \\
\hline Base2 & 314.0 & 312.5 & 313.1 & 312.0 & 308.1 \\
\hline Int1 & 9.5 & 15.5 & 12.9 & 14.1 & 17.1 \\
\hline Int2 & 2.1 & 2.0 & 1.9 & 4.0 & 3.9 \\
\hline Int3 & 21.0 & 16.8 & 18.6 & 16.8 & 17.0 \\
\hline Int4 & 2.9 & 2.6 & 3.1 & 2.4 & 3.4 \\
\hline Int5 & 0.1 & 0.2 & 0.1 & 0.3 & 0.3 \\
\hline Peak1 & 0.3 & 0.2 & 0.2 & 0.3 & 0.4 \\
\hline Peak2 & 0.0 & 0.0 & 0.0 & 0.1 & 0.1 \\
\hline Peak3 & 0.0 & 0.0 & 0.0 & 0.0 & 0.0 \\
\hline
\end{tabular}

Table 4-4 and Table 4-5 summarize operating costs and other main results in ED. We can see, as expected, that $\mathrm{F} 1$ has the lowest total operating cost because it assumes that a perfect wind power forecast is available. In other words, the dispatch from the UC run does not need to change in the RT ED. F2 has the highest cost as a result of the high load curtailment, which is caused by WPF errors. Both of the stochastic cases have relatively low operating costs, and the lowest cost occurs in S2. It is interesting to note that R2 performs slightly better than S1 in terms of the operating cost. This result occurs because the additional reserve requirement was derived from the WPF information, which leads to bringing more units on-line to provide the ramping capability to accommodate the forecasting errors. The column of reserve curtailment in Table 4-5 shows the amount of curtailed reserve in the various cases. Because we assume the reserve requirement is in the form of a demand curve, and the cost of unserved reserve is lower than the cost of unserved load, a certain amount of reserve will be curtailed ahead of system load when system reliability is hampered. Most cases have some unserved reserve, whereas only F2 and F5 have unserved load. 
Table 4-4 Summary of operating costs.

\begin{tabular}{|c|c|c|c|c|c|}
\hline Case & Description & $\begin{array}{c}\text { Fuel } \\
\text { Cost } \\
{[\mathrm{M} \$]}\end{array}$ & $\begin{array}{c}\text { Load } \\
\text { Curtailment } \\
\text { Cost } \\
{[\mathrm{K} \$]}\end{array}$ & $\begin{array}{c}\text { Reserve } \\
\text { Curtailment } \\
\text { Cost } \\
{[\mathrm{M} \$]}\end{array}$ & $\begin{array}{c}\text { Total } \\
\text { Dispatch } \\
\text { Cost } \\
{[\mathrm{M} \$]}\end{array}$ \\
\hline F1 & Perfect forecast & 34.86 & 0 & 0.00 & 34.87 \\
\hline F2 & Point forecast & 34.78 & 282 & 13.02 & 48.08 \\
\hline F3 & No forecast & 36.81 & 0 & 0.00 & 36.81 \\
\hline F4 & $\begin{array}{c}20 \% \text { quantile } \\
\text { forecast }\end{array}$ & 35.11 & 0 & 2.49 & 37.59 \\
\hline F5 & $\begin{array}{c}50 \% \text { quantile } \\
\text { forecast }\end{array}$ & 34.86 & 574.87 & 7.70 & 43.14 \\
\hline R1 & Wind pf reserve & 35.06 & 0 & 5.05 & 40.11 \\
\hline R2 & $\begin{array}{c}\text { Wind quantile } \\
\text { reserve }\end{array}$ & 35.26 & 0 & 0.86 & 36.12 \\
\hline R3 & Load reserve & 35.19 & 0 & 1.42 & 36.61 \\
\hline S1 & Stochastic UC & 35.34 & 0 & 1.05 & 36.39 \\
\hline S2 & $\begin{array}{c}\text { Stochastic }+ \\
\text { reserve }\end{array}$ & 35.83 & 0 & 0.06 & 35.90 \\
\hline
\end{tabular}

Table 4-5 Summary of other results.

\begin{tabular}{|c|c|c|c|c|}
\hline Case & Description & $\begin{array}{c}\text { Load } \\
\text { Curtailment } \\
{[\mathrm{MWh}]}\end{array}$ & $\begin{array}{c}\text { Reserve } \\
\text { Curtailment } \\
{[\mathrm{MWh}]}\end{array}$ & $\begin{array}{c}\text { Average } \\
\text { Available } \\
\text { Reserve } \\
{[\mathrm{MW}]}\end{array}$ \\
\hline F1 & Perfect forecast & 0 & 0.57 & 178 \\
\hline F2 & Point forecast & 80.64 & 0.01 & 172 \\
\hline F3 & No forecast & 0 & 0.00 & 294 \\
\hline F4 & $\begin{array}{c}20 \% \text { quantile } \\
\text { forecast }\end{array}$ & 0 & $2,259.11$ & 194 \\
\hline F5 & $\begin{array}{c}50 \% \text { quantile } \\
\text { forecast }\end{array}$ & 164.25 & $7,003.10$ & 176 \\
\hline R1 & Wind pf reserve & 0 & $4,588.75$ & 189 \\
\hline R2 & $\begin{array}{c}\text { Wind quantile } \\
\text { reserve }\end{array}$ & 0 & 781.5 & 204.5 \\
\hline R3 & Load reserve & 0 & $1,288.42$ & 199 \\
\hline S1 & Stochastic UC & 0 & 952.91 & 207 \\
\hline S2 & Stochastic + reserve & 0 & 58.78 & 237 \\
\hline
\end{tabular}

\subsubsection{Use of Different WPFs}

In subgroup 1, various WPFs are used. The total cost ranges from $\$ 34.87 \mathrm{M}$ in F1 to $\$ 48.08 \mathrm{M}$ in F2. The total cost in F3 is lower than the operating cost in F2 as a result of F2's high curtailment cost. The difference between F2 and F3 illustrates the importance of avoiding extreme errors in WPF, where the wind power forecast has a very large magnitude or phase error compared to the realized wind power generation. This event could occur within one hour or a limited number of hours even though the average forecast error over the entire period may be small. In fact, load curtailment occurs in only four hours out of the 91-day simulation in F2. Nevertheless, reserve curtailment occurs as often as $17 \%$ of the time in F2.

Forecasting errors also lead to reserve curtailment. In these situations, the system operator is assumed to use the RT operating reserve intended for unexpected deviations in load and thermal generation to accommodate the WPF errors. These events cause great challenges for system 
operations because the generating units are not scheduled accordingly, and sufficient ramping capability is therefore not available. However, F3 ends up with fuel costs that are higher than they are in any of the other scenarios, because more high-cost generating units are being dispatched compared to what is needed, given that wind power is not considered at all in the UC problem.

F4 is the $20 \%$ quantile forecast, which means that the realized wind power is higher than this forecast with a probability of $80 \%$, and is used in place of the point forecast in the UC. The commitment results show that more units are committed in F4 compared to F2 because of using the conservative forecast for wind power. As a result, the curtailment cost and the total operating costs are both much lower in F4 than in F2. The operating cost in F5 is much higher than it is in $\mathrm{F} 3$ and F4, which shows that it is too risky to rely on the $50 \%$ quantile forecast in the UC.

\subsubsection{Reserve Requirements}

Subgroup 2 compares the impact of the reserve requirements on the system dispatch. The additional reserve in this subgroup is meant to compensate for the WPF error in a predefined manner. This predefined amount could be inaccurate as discussed above. The total hours of commitment in Fig. 4-4 show that the three reserve requirements have a similar effect on the commitment results because more units are required to be on-line to provide extra reserve.

However, case R1 suffers from the wind power point forecast errors, to some extent, because the additional reserve requirement is a function of the point forecast. If the forecast deviates substantially from the realized wind power, the resulting reserve may not be sufficient to handle the forecast error. In this subgroup, R2 uses the best strategy. In this case, the possible forecasting errors are largely compensated for by using the larger extra reserve, which is the difference between the point forecast and the $10 \%$ quantile forecast. The operating cost in R3 turns out to be slightly higher than that of R2 because the reserve requirement in R3 is linked with the system load, not the WPF. Thus, because the load is not strongly correlated with the wind power output, the reserve associated with load may be incorrect.

In comparison to subgroup 1, the strategies in R2 and R3 lead to lower costs than those of F3 and F4, where no forecast or a conservative forecast is used. This result shows, as expected, that increasing the reserve requirements in the deterministic approach has the effect of better accommodating wind variability and uncertainty. The resulting operating reserve is higher in R2 and R3 compared to all of the cases in subgroup 1 except F3 (Table 4-5). Because F3 can be regarded as the most conservative way to commit the units - that is, by ignoring the existence of wind power - the level of realized operating reserves is therefore much higher than it is in the other cases.

\subsubsection{Deterministic vs. Stochastic UC}

Fig. 4-4 shows that the units are on more frequently in S1 and S2 than in the deterministic cases, except in F3 where there is no wind power forecast. This result occurs because the units need to be on to provide the additional ramping capacity to deal with the variability of wind power output in the scenarios in the stochastic approach.

In the stochastic cases S1 and S2, we can see that the total operating costs end up being close to deterministic case F3, which has no wind power forecast, and cases R2 and R3, which have 
additional operating reserves. S2 shows the best performance in terms of the total cost among all of the cases because both the wind power variability in the scenarios and the limited accuracy of the scenario generation are taken into account. For the same reason, the available reserve is also relatively higher in S2. This observation is consistent with [9], where the increased reserve is used to cover the additional generator outages and load variations, which are not captured by the scenarios.

The results show that the UC strategy and the reserve requirements have important implications for managing the costs and reliability of power systems with large amounts of wind power. The ramping capability and reserves provided by on-line units are, to a large extent, determined by these factors, which therefore influence the RT dispatch results to a great extent. Again, it should be noted that the RT dispatch can be further improved if intra-day rescheduling of generating units is applied, which is not considered in this chapter.

\subsection{Conclusions}

This chapter analyzes the impact of WPF on UC and ED. More than elaborating on theoretical models, it is important to assess whether the difference in decisions, resulting from moving from deterministic models to stochastic programming models, is relevant enough to justify the attention of the industry to changing the way they operate the system. Therefore, the studies reported in the chapter were carefully organized in a series of experiments to cover a whole set of strategic variants. The results show that the added uncertainty resulting from WPF uncertainty requires the adoption of an improved UC policy that can define higher spinning reserves than those used in classical systems that do not rely on wind resources. This result is of major economic impact to system operators.

The simplest approach to this requirement of setting a larger reserve margin is to define a larger fixed value (in terms of percentage of the load) for the spinning reserve - so that not only errors in WPF but also in load forecast and the possibility of unplanned outages are covered. Our results show, however, that an adaptive strategy to define adequate reserves should be put in place, because WPF errors vary importantly from hour to hour. Our results also show that a probabilistic wind power forecast can be used to define more adequate reserve requirements.

In addition, the example shows that the desired level of reserves is more likely to be achieved with a stochastic UC formulation that explicitly takes into account the varying uncertainty in wind power predictions. One of the major contributions of this chapter is to adopt scenarios with temporal correlation of the errors within each scenario when applied to the UC problem. Most approaches published to date have been using Gaussian representations of WPF uncertainty that are in the form of marginal distributions, which do not represent time dependency ${ }^{13}$ - a treatment that results in gross approximations, because the UC problem also has a strong timedependency component in its definition (ramping, shut-down/start-up decisions, and, if hydro power is present, the use of water). A stochastic UC formulation with representative scenarios is clearly better, because it can handle the temporal correlation in forecasting errors as opposed to explicit reserve requirements.

\footnotetext{
${ }^{13}$ An exception is the WILMAR model, which does not use a Gaussian representation of WPF uncertainly, but uses an approach taking the temporal correlations of the forecast errors into account [66].
} 
In conclusion, the chapter sustains that system operators may move with confidence to the adoption of probabilistic (stochastic) decision methods in order to reduce costs and risks. In fact, the adoption of a "blind" fixed criterion for the reserve tells nothing about the credibility of its assumptions or about the risks. It may happen that in some instances, a fixed reserve value may approximate the results from a stochastic decision process - however, one can never be certain about this beforehand. In the presence of the large and unavoidable wind power prediction errors, especially in thermal-dominated power systems, stochastic approaches should prove to be more useful. A first step would be to move toward the use of adaptive reserve requirements, which are a function of the wind power forecast. Next, operators can deploy an entirely stochastic approach, in which the wind power forecast uncertainty is fully represented in the UC formulation. 
This page intentionally blank. 


\section{REPRESENTATIVE WIND POWER SCENARIOS AND THEIR PROBABILITIES FOR STOCHASTIC MODELS ${ }^{14}$}

This chapter analyzes the application of clustering techniques for wind power scenario reduction. The results show the unimodal structure of scenarios generated under a Monte Carlo process. The unimodal structure has been confirmed by the modes found by the information theoretic learning Mean Shift algorithm. The chapter also presents a new technique that is able to represent the WPF uncertainty by a set of representative scenarios capable of characterizing the probability density function of the wind power forecast. From an initial large set of sampled scenarios, a reduced discrete set of representative scenarios associated with a probability of occurrence can be created finding the areas of high-probability density. This discrete set will allow the reduction of the computational burden in stochastic models that require scenario representation, such as the stochastic UC models presented in Chapter 4.

\subsection{Introduction}

The continuous increase of wind power penetration is introducing more variability and more volatility in electricity generation. This challenges the management and planning of the electric power system. To cope with uncertain wind power production, efforts are constantly invested in developing more accurate wind power forecasts. A common form of delivering the wind power forecast is called point forecast and represents a single value for each look-ahead time horizon. However, no forecast can be perfect. When the forecast is delivered as a point forecast, it commonly contains only the conditional expectation at each time step. However, an expectation cannot help in calculating risks or the conditional values at risk: one needs a description of the probability density function (pdf).

The power industry is in need of estimates of forecasting uncertainty, besides the point forecasts. The most common description has been in the form of marginal distributions for each time step. The presentation of these results, usually, is done in the form of intervals or quantiles, but this is a somewhat misleading form of presentation, because it gives the illusion of "trajectories" or time sequences. However, at each time step what one has is a marginal distribution, regardless of what happened in the previous time steps — the time dependency is absent from the interval/quantile representation.

However, for some applications, one cannot neglect time dependencies: it is the case, for instance, of UC decisions to be made by system operators. Because of technical constraints, such as ramping limits or minimum shutdown and start-up times, and because of the nature of costs, the decisions to be made also involve time dependencies.

The traditional practice calls for deterministic models to be used by system operators in the decision-making process. This was acceptable when the uncertainties were of limited amplitude, such as in load forecasting. However, wind generation uncertainty tends typically to be significantly greater than load forecasting uncertainty. Hence, the description of uncertainty must be incorporated in the models.

\footnotetext{
${ }^{14}$ This chapter is reproduced from Proceedings $16^{\text {th }}$ Int. Conference on Intelligent System Application on Power Systems (ISAP), J. Sumaili, H. Keko, V. Miranda, Z. Zhou, A. Botterud, J. Wang, "Finding Representative Wind Power Scenarios and their Probabilities for Stochastic Models," Greece, Sept. 2011, with permission from IEEE.
} 
Because the decision models are usually based on mathematical programming approaches, the logical way to include uncertainty in a multiple time step optimization process is to adopt stochastic programming models. These models usually work with scenarios associated with probabilities. In the case of WPF, a scenario is a time series or a particular sequence of values representing an assumed possible realization of wind power along some period. A thorough description of wind power uncertainty would be by a probability density function in a space of multiple dimensions (the number of time steps within the prediction horizon). An approximate representation of this pdf can be obtained with a set of scenarios, sampled from the probability density function representing the historical (observed) error distribution. While this method is favorable since it is able to carry the information on the temporal development of uncertainty, the primary drawback of the scenario representation is its implied computational burden: a high number of scenarios is needed to accurately represent the associated uncertainty. This means that the decision making involving stochastic optimization may turn prohibitively hard for such a large set of scenarios.

In this chapter, we analyze a technique of representing the wind power forecast related uncertainty with a reduced set of scenarios. The development of a reduced scenario set is based on clustering methods. Within the process of reducing the initial large set of scenarios, the newly obtained scenarios are also associated with the probability related to the cluster it represents. The newly obtained set of representative scenarios could then be applied to computationally heavy problems.

The proposed methodology assumes departing from a large set of wind power scenarios generated using a Monte Carlo sampling based method. Each scenario is actually a Monte Carlo sample or a point in a space whose dimension is equal to the number of time steps considered. This large set of scenarios should be a suitable approximation of the probability density function of wind power. One of the possible approaches to generate these scenarios is presented in [78].

The high-density areas are substituted by a single representative or focal scenario. Departing from the reasoning that the initial samples are sampled by using a Monte Carlo process, the relative weight of each high-density area with regard to all of the samples becomes the probability of the representative scenarios representing that area.

In this work, the scenarios sampled by using the process described in [78] are analyzed and the structure of such scenarios is shown to be unimodal, by means of using the information theoretic learning (ITL) Mean Shift algorithm [86], [87].

The goal of the presented work is to find an adequate representation of the shape of the probability density function by using a reduced set of scenarios with assigned probabilities. Having the assigned probabilities makes the proposed reduction method adequate for risk assessment. The scenario reduction topics from the literature commonly use the scenario tree construction method from [88], [89]. This work instead uses a clustering approach without assuming any particular temporal development characteristic of errors. Given a set of initial scenarios, the reduction level is only determined by the tolerance admitted. 
Depending on the particular power system characteristics, the wind forecast uncertainty may carry large hedging costs. The reduced set of scenarios obtained by clustering is a discrete representation of the probability density function using a smaller weighted set of wind power scenarios. Such a smaller set of scenarios may increase the feasibility of using risk assessment models in a practical setting, which is favorable for managing the hedging costs.

This chapter describes the details of the model developed and presents the results for three DA forecasted wind power scenarios, in a study case built with realistic data. The use of scenario reduction methods for stochastic UC is further explored in Chapter 6.

\subsection{Scenario Generation and the Need for Scenario Reduction}

The initial sets of scenarios used refer to DA wind power forecasts in 2006 for a hypothetical wind farm located in the state of Illinois. Time series of DA deterministic point forecasts were obtained from the Eastern Wind Integration and Transmission Study [83].

To produce wind power scenarios, wind power data (forecasted and realized) were used to train the uncertainty estimation model, as well as to generate scenarios for the forecasted wind power, according to the methodology introduced by Pinson et al. [78], which is equivalent to producing scenarios under a Monte Carlo process.

The work presented shows how one can organize a large set of scenarios into a small set of clusters, based on a principle of maximum density; associate each cluster with an empirical probability value; and represent each cluster by a focal or representative scenario. This technique allows the organization of a cumulative distribution of clusters. This permits not only the selection of the most representative equivalents but also keeps a quantitative (probability) evaluation of the coverage of possibilities guaranteed - and, of course, of the risk of missing the actual wind power series.

\subsection{Wind Power Scenario Clustering and Modes Finding}

\subsubsection{Application of Classical Clustering Techniques to Wind Power Scenarios}

We apply the classical pattern clustering techniques in order to identify high-probability density areas characterized by similar wind power scenarios.

The hierarchical clustering methods rely on a measure of similarity (for agglomerative clustering), in order to build up the clusters from the samples. One starts with the lowest level of hierarchy where each cluster contains a single member, that is, the number of clusters is equal to the number of scenarios and merges (agglomerates) progressively the two most similar clusters [90]. At the very highest level of hierarchy, there is a single cluster containing all of the data. At the intermediate levels of hierarchy, a common behavior of the hierarchical clustering methods is to group the samples into a single relatively large cluster, while the rest of the clusters contain outliers. This behavior is observed for the wind power scenarios generated according to [78].

On the other hand, the classic K-means algorithm [91] leads to the separation of the initial scenario set into different clusters; however, this clustering method maintains relatively high dispersion inside the clusters. This is due to the unimodal structure of the initial scenario set generated by the model [78] behind the Monte Carlo sampling. 


\subsubsection{Modes Finding using the ITL Mean Shift Algorithm}

The hypothesis that the initial scenario distribution produced by the technique proposed in [78] is unimodal is confirmed by using the ITL Mean Shift algorithm [86], [87]. The Mean Shift is a nonparametric, mode-finding method that relies on a kernel-based construction of the search space surface.

The mode-finding method was applied to confirm that the actual distribution represented by the initial set of scenarios is unimodal, which seems to present a difficulty for the classic clustering methods, as discussed in the previous subsection. When the Mean Shift algorithm is applied to a single-day data set, it finds just a single mode, confirming the hypothesis.

A test of the ability of the ITL Mean Shift algorithm to deal with multimodal wind scenario distributions was designed, as well. A total of 1,000 scenarios from three different days (Fig. 5-1 through Fig. 5-3) were used as the input data set, totaling 3,000 scenarios. Each of the separate days should have a single mode, and the three-day data set, when merged together as if it were a single day, should then be three-modal.

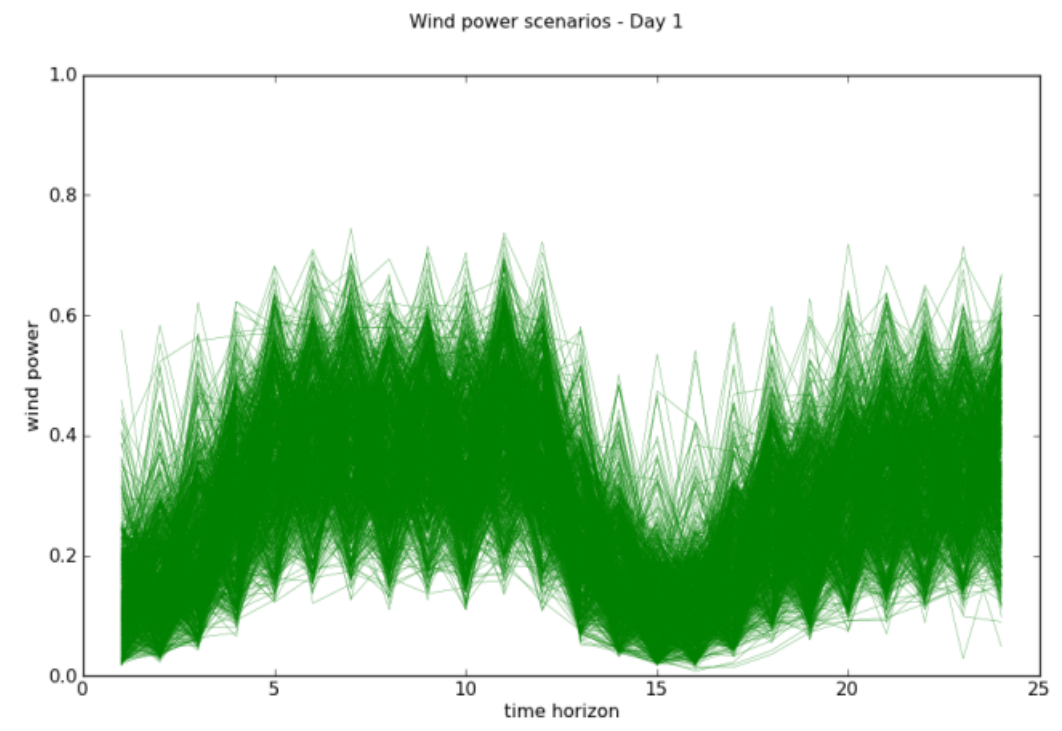

Fig. 5-1 Day 1 wind power scenarios. 
Wind power scenarios - Day 2

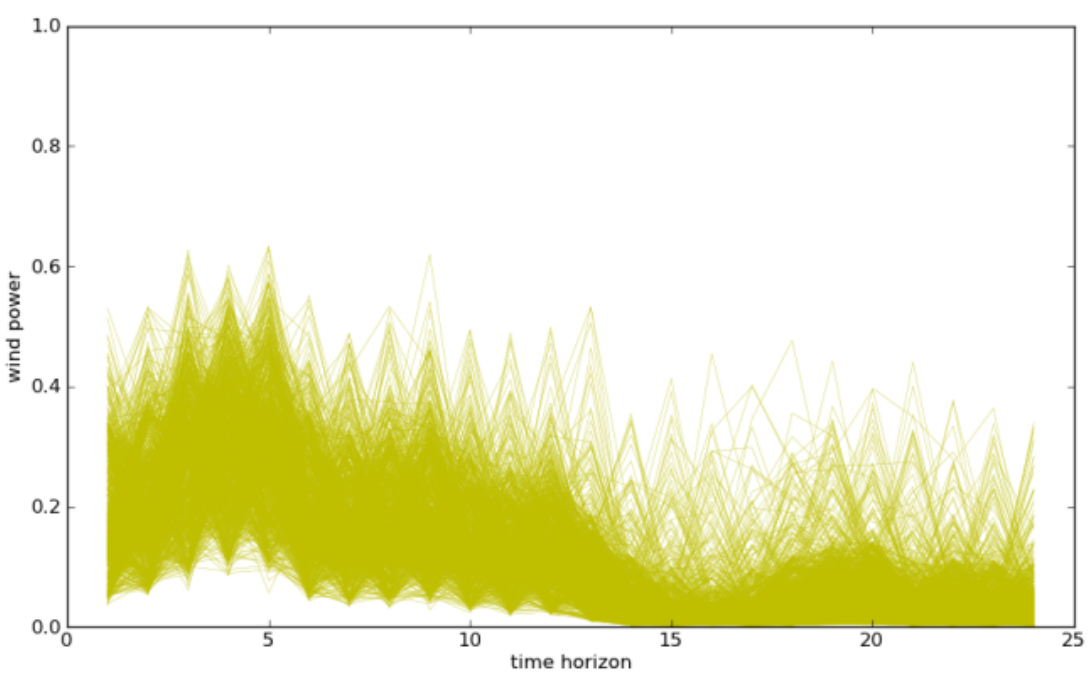

Fig. 5-2 Day 2 wind power scenarios.

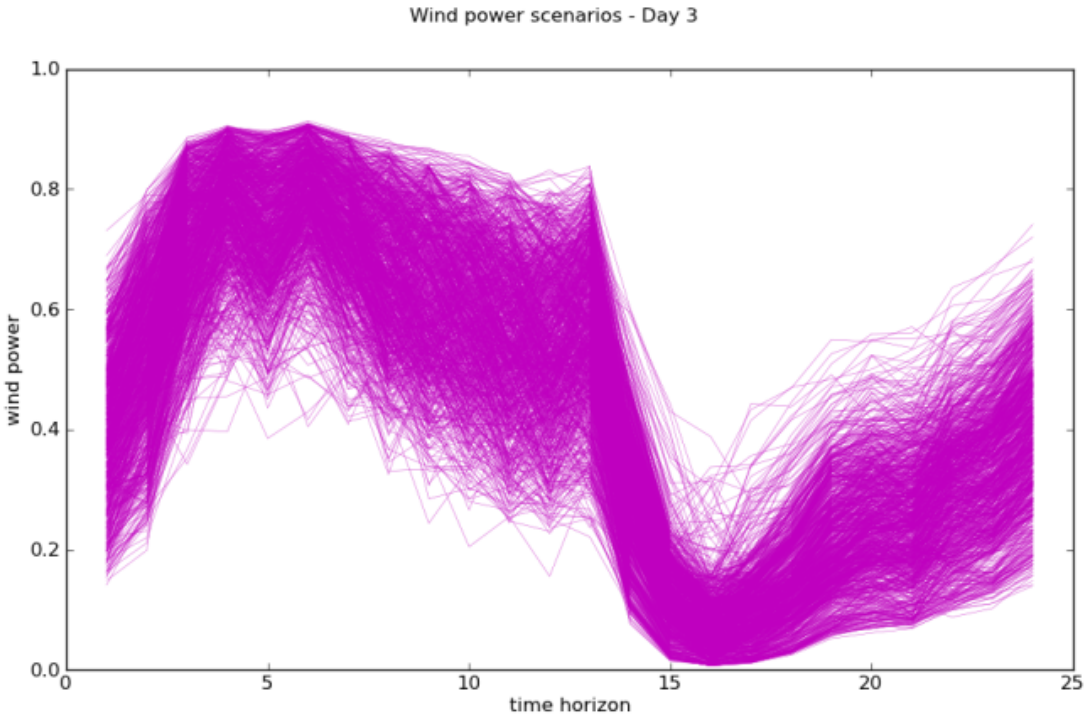

Fig. 5-3 Day 3 wind power scenarios.

Fig. 5-4 illustrates the results of the ITL Mean Shift algorithm with $\lambda=1$ and $\sigma=0.18$ (for details of the algorithm, refer to [86][87]). 


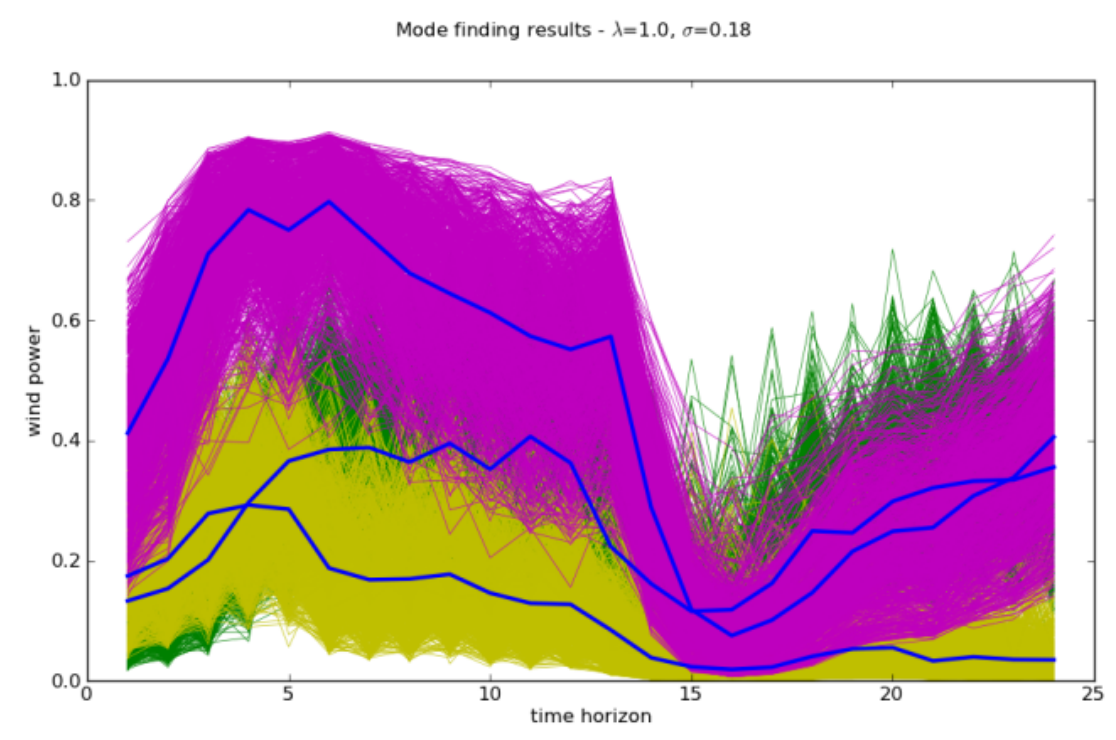

Fig. 5-4 Day 3 Mode finding results for the three-day set of scenarios - thick lines represent the modes discovered, coherent with the merged sets.

The ITL Mean Shift algorithm was successful in finding the three modes in the merged scenario set resulting from joining the three days into a single set. Each mode is represented by a thick line, and it represents the three main evolutionary trends present in the global scenario set.

This property may suggest that the ITL Mean Shift algorithm can be used effectively when the set of scenarios is produced from ensembles and merged - the information is not lost and can be recovered.

\subsection{New Methodology}

Given a set of $\mathrm{M}$ wind power scenarios sampled according to some probability density function describing the uncertainty in forecasts, the aim of the reduction process is to merge scenarios presenting high similarity in order to produce a new reduced set, containing the representative scenarios only.

Each set of merged scenarios forms a cluster and is associated to a certain frequency within the sample - its representative or focal scenario is therefore associated with the same probability value.

Let us denote the forecasted wind power as a series:

$$
\mathrm{p}^{(\mathrm{m})}=\left\{\mathrm{p}_{1}{ }^{(\mathrm{m})}, \ldots, \mathrm{p}_{\mathrm{T}}{ }^{(\mathrm{m})}\right\},
$$

for time horizon $\mathrm{t}=1, \ldots, \mathrm{T}$ in scenario $\mathrm{m}=1, \ldots, \mathrm{M}$. This is also a point in a space of dimension $\mathrm{T}$.

The forecasted wind power scenarios $\mathrm{p}^{(\mathrm{m})}$ are normalized with respect to the installed power of the wind farm (or generator) in order to obtain the vectors $\mathrm{x}^{(\mathrm{m})}$, whose components lie in the 
range $[0,1]$. Alternatively, this range can be changed to $[0,100]$ if the forecasted power is expressed as a percentage of the installed power.

Different metrics can be used to define the distance between two scenarios $\mathrm{x}^{(\mathrm{i})}$ and $\mathrm{x}^{(\mathrm{j})}$. For the work presented in this paper, the maximum deviation has been used:

$$
d_{\max }\left(x^{(i)}, x^{(j)}\right)=\max _{t}\left\|X_{t}^{(i)}-X_{t}^{(j)}\right\|, t=1, \ldots, T
$$

where $\mathrm{x}^{(\mathrm{i})}$ and $\mathrm{x}^{(\mathrm{j})}$ are the two scenarios that are being compared, and $\mathrm{T}$ is their dimension.

The reduction procedure begins with an extremely large set of wind power scenarios, and then one finds the scenario presenting the highest number of neighbors according to a defined tolerance $\varepsilon$. Two scenarios $\mathrm{x}^{(\mathrm{i})}$ and $\mathrm{x}^{(\mathrm{j})}$ are considered to be neighbors if the following is satisfied:

$$
\mathrm{d}_{\max }\left(\mathrm{x}^{(\mathrm{i})}, \mathrm{x}^{(\mathrm{j})}\right) \leq \varepsilon
$$

The identified scenario along with its neighbors are then cut from the initial set and grouped in a cluster. The process is repeated with the remaining part until the scenarios do not have any neighbor within the defined tolerance. In this way, a set of clusters is defined. Each of them is associated with a focal element together with a probability value.

For this method, it is necessary to define a tolerance for the scenario aggregation process. The value of this tolerance is a trade-off between accuracy and set reduction capability, which is related to the computational effort.

After identifying a cluster and its members, the attracting scenario is taken as the representative or focal scenario of the cluster and replaces the cluster as a condensed discrete representation. Another option is to calculate the mean value of the cluster to obtain the centroid of the cluster. This scenario is thus produced by averaging the cluster members. A third option is to apply the ITL Mean Shift algorithm to find the mode within the cluster. In these latter cases (average or mode), the result is usually not an existing scenario.

One should be careful in choosing the options for calculating the representative scenario. Averaging the members of the cluster may artificially introduce smoothing effects in the new "artificial" scenario. This "average" scenario would not have been generated by the initial scenario-generation process, so its behavior may not be in line with the original probabilistic model that captured the predicted behavior of the wind power.

On the other hand, if a cluster member is chosen as the representative scenario, therefore becoming the focal element, the total expected value may deviate from the expected value of the whole set of scenarios. The preferred method for the focal scenario calculation thus depends on the purpose of the scenario reduction.

Each focal scenario is then associated with a probability value, calculated as the ratio between the number of scenarios in the cluster it represents and the total number of scenarios. Finally, after choosing the representative scenarios and calculating the corresponding probabilities, the 
continuous probability density function of wind power initially represented by a large discrete sample becomes represented by a much smaller discrete set of focal scenarios, each with a probability value assigned.

\subsection{Results}

The methodology described above was applied to the sets of 1,000 scenarios for the three days illustrated in Fig. 5-1 through Fig. 5-3 in Section 5.3.2. The scenario reduction results are summarized in Fig. 5-5 through Fig. 5-7 below.
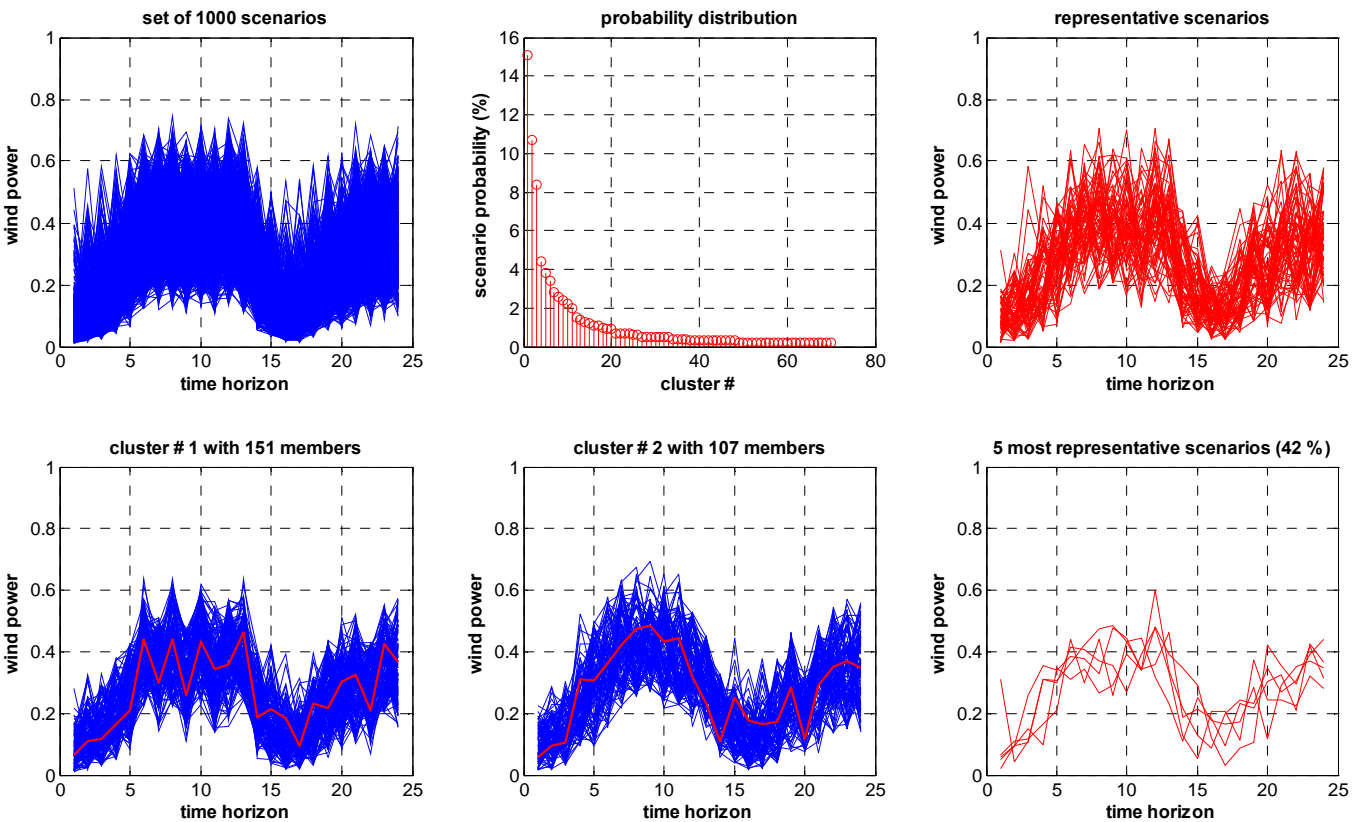

Fig. 5-5 Day 1 scenario reduction results with $\varepsilon=0.22$.
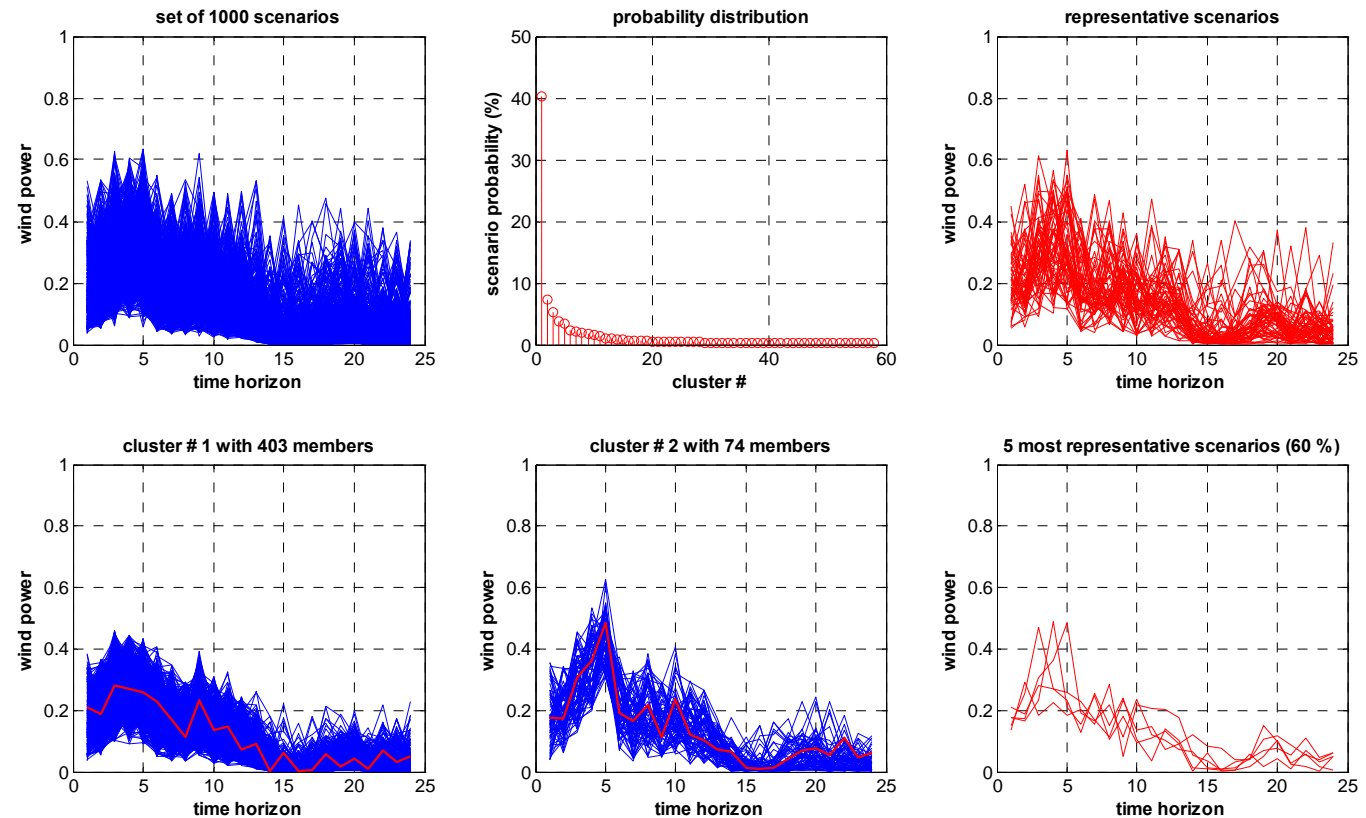

Fig. 5-6 Day 2 scenario reduction results with $\varepsilon=0.18$. 

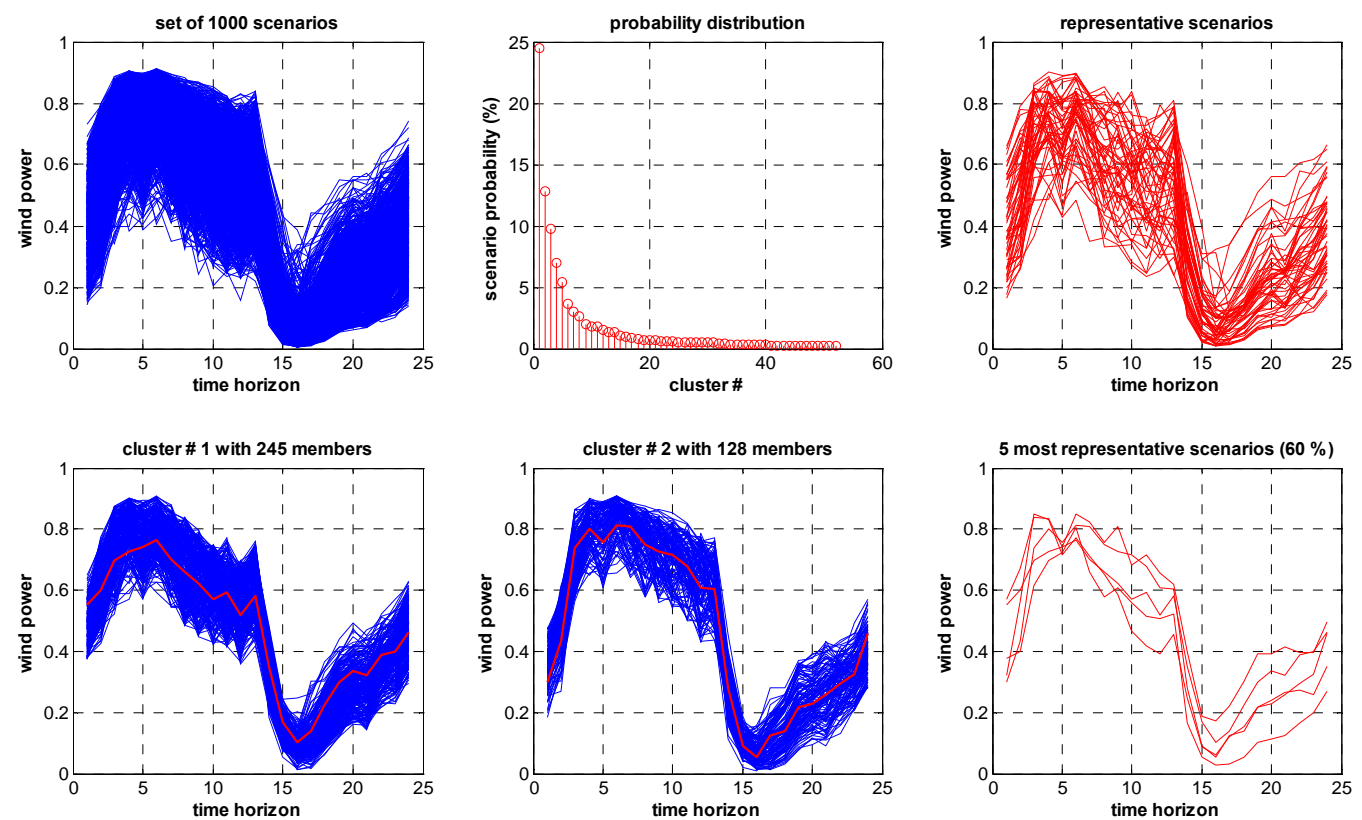

Fig. 5-7 Day 3 scenario reduction results with tolerance $\varepsilon=0.18$.

On the top line, the three windows illustrate the initial set of scenarios; the probability distribution associated to the clusters, ordered by decreasing probability value; and the whole set of representative scenarios, obtained with the full distilling of the data set. On the lower line, the two first windows illustrate the two more populated clusters with their corresponding focal scenarios, and the last figure presents the five most representative focal scenarios (with highest probability). The coverage of the possibilities, when only these five scenarios are retained, is indicated.

With the admissible tolerance $\varepsilon$ set to 0.22 , the initial 1,000 -scenario set of day 1 has been reduced to 70 clusters and 150 outliers (Fig. 5-5). The 70 clusters represent $85 \%$ of the total number of scenarios. The five most representative scenarios represent only about $42 \%$ of the 1,000 initial scenarios.

If one decides to run a stochastic model only with the five most representative focal scenarios, one is missing $58 \%$ of the scenario representation, which may be considered as a risk measure the risk of missing the adequate representation of wind power, for accepting the 5 focal scenarios as representative of the real distribution. To reduce the risk, one has to consider the addition of more scenarios. Naturally, this addition should be in an order following a decreasing value of the probability assigned to each cluster.

Fig. 5-6 and Fig. 5-7 present results for days 2 and 3 with tolerance $\varepsilon$ set to 0.18 . The scenario reduction procedure has achieved 58 representative scenarios and 112 outliers (the five most representative scenarios contain about $60 \%$ of the 1,000 initial scenarios) for day 2 and 52 representative scenarios and 50 outliers (the five most representative scenarios contain about $60 \%$ of the 1,000 initial scenarios) for day 3 .

The number of obtained representative scenarios depends on the tolerance value $\varepsilon$ and on the dispersion of the initial scenario data set. Fig. 5-8, Fig. 5-9, and Fig. 5-10 illustrate the distribution of the maximum deviation between any pair of the original scenarios for days 1,2 , 
and 3, respectively. Notice that day 1 presents the lowest number of pairs of scenarios displaying a maximum deviation lower than 0.1 ; in other words, day 1 has a lower number of similar scenarios according to the maximum deviation distance metric. This result sets the base and rationale for defining tolerance values $\varepsilon$ with a distinct value for different days.

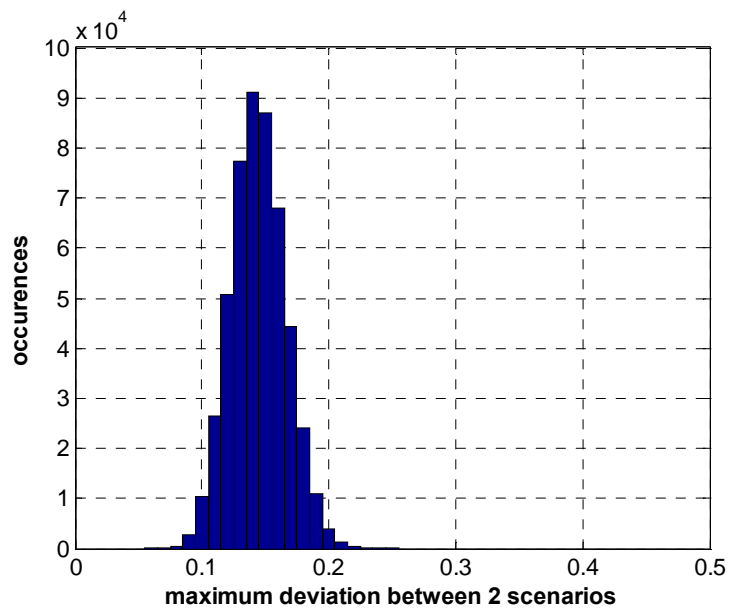

Fig. 5-8 Day 1 distribution of the maximum deviation between each pair of 2 scenarios.

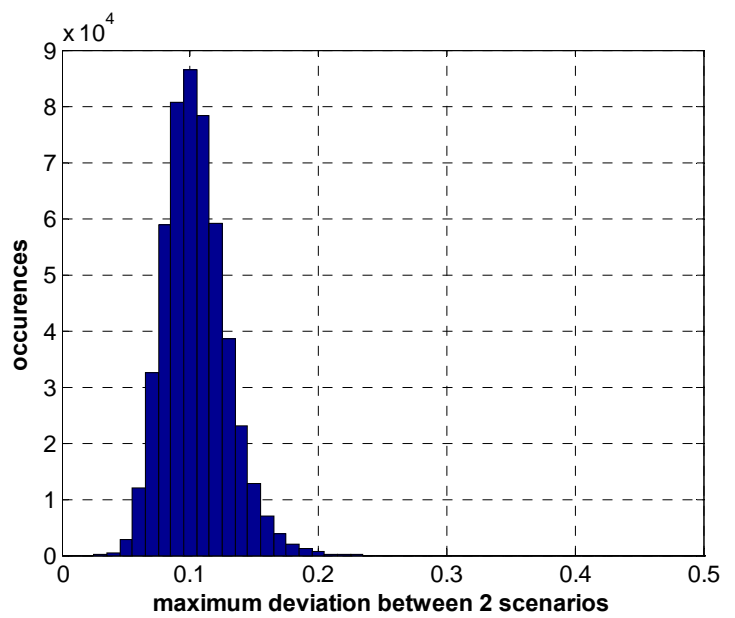

Fig. 5-9 Day 2 distribution of the maximum deviation between each pair of 2 scenarios.

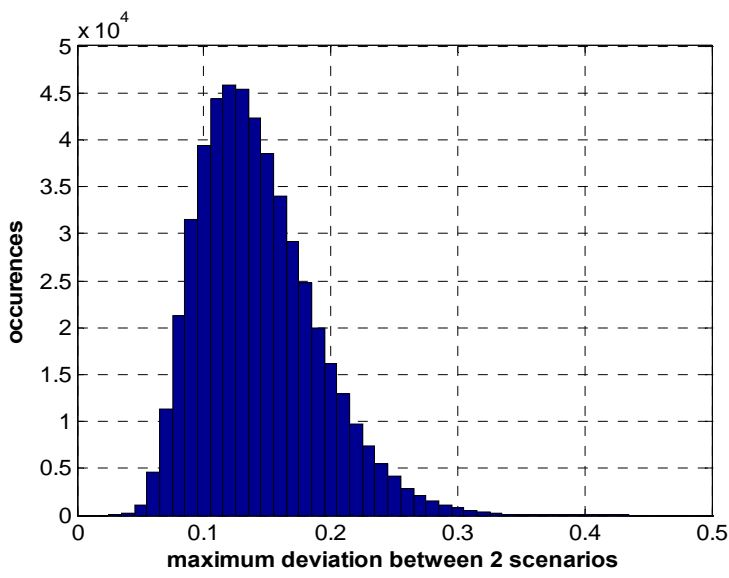

Fig. 5-10 Day 3 distribution of the maximum deviation between each pair of 2 scenarios. 


\subsection{Conclusion}

Decision-making processes in the operation of a power system must take into account the uncertainty in wind power prediction. The most convenient way to represent this uncertainty is by a set of scenarios.

A discrete approximation of the probability density function representing the uncertainty in WPF may be provided by a sampling procedure of the Monte Carlo type. However, to have an accurate representation of the probability density function covering all of the forecasting space, an extremely large set of elementary scenarios is needed. But the size of this set is usually incompatible with the computational complexity of stochastic programming algorithms, which are time demanding and require high computational effort.

The methodology proposed in this paper can substitute a large scenario set by a smaller set of clusters, each one being replaced by a representative scenario associated with the probability of the cluster it represents.

The reduction capability depends on the admitted tolerance, the distance metric used to define the clusters, and the scenario dispersion. The choice of the metric and the admissible tolerance has to be defined in accordance with the problem for which the scenario reduction is being made and the level of accuracy needed for the results.

The method not only produces a set of focal or representative scenarios, but also orders them by probability value. This allows retaining a selected number of scenarios and understanding the risk of missing an adequate representation for truncating the scenario set. It also allows one to choose the necessary number of scenarios in order to satisfy a maximum level of risk.

This work, therefore, is a contribution to facilitating the adoption of stochastic optimization models in operation planning, a move that is seen as necessary to embed the uncertainty in WPF in the system operators' decision-making processes.

Stochastic optimization models for UC and wind power trading are presented and applied in Chapters 4, 6, and 7. 
This page intentionally blank. 


\section{UNIT COMMITMENT AND OPERATING RESERVES WITH PROBABILISTIC WIND POWER FORECASTS ${ }^{15}$}

This chapter expands on the previous two and discusses in more detail how probabilistic wind power forecasts can serve as an important tool to efficiently address wind power uncertainty in power system operations. In particular, we compare different probabilistic forecasting and scenario reduction methods, and test the resulting forecasts on the stochastic UC model presented in the Chapter 4. The results are compared to deterministic UC, where dynamic operating reserve requirements can also be derived from the probabilistic forecasts. In both cases, the use of probabilistic forecasts contributes to improve the system performance in terms of cost and reliability.

\subsection{Introduction}

The increasing penetration of wind power and other renewables are posing new challenges to power system operators in grid management and generation scheduling. The inherent uncertainty and variability from wind power calls for new approaches to the traditional UC and ED problems. Stochastic UC has been proposed as one approach to better handle the wind power uncertainty in market operations [55]-[58] and [66]-[68],[70]. Furthermore, new approaches to calculating operating reserve requirements under wind power uncertainty have also been proposed (e.g., based on assumed normal distributions of WPF errors [29][48], or on a probabilistic wind power forecast [75]). It is obvious that WPF can provide important inputs to the operational decisions of the system operator. However, probabilistic WPF is still a relatively new area of research, and there is limited experience in the use of such forecasts in system operations so far.

In order to investigate the consequences of using different UC strategies and reserve requirements in the operation of the electricity market, we have developed a stochastic UC model [60][86] as presented in Chapter 4. The stochastic model determines the commitment of thermal generating units by minimizing the expected cost over a set of WPF scenarios. Alternatively, quantiles from probabilistic WPF can be used to determine operating reserve requirements in a deterministic UC. In either case, the quality of the probabilistic forecasts is clearly of major importance for the cost effectiveness and reliability of the resulting scheduling and dispatch.

In this chapter, we use the model to analyze the scheduling of energy and operating reserves under wind power uncertainty. We compare different statistical methods for the generation of probabilistic forecasts and also compare two different scenario reduction approaches. Furthermore, we compare the use of stochastic and deterministic UC strategies and operating reserves requirements. The main contribution of the chapter is in the systematic analysis of probabilistic WPF methods and their potential use in power system operations. The results and recommendations are highly relevant for ongoing efforts to improve the management of wind power uncertainty and variability in electricity markets.

\footnotetext{
${ }^{15}$ This chapter is based on Proceedings IEEE Trondheim PowerTech 2011, A. Botterud, Z. Zhou, J. Wang, J. Valenzuela, J. Sumaili, R.J. Bessa, H. Keko, V. Miranda, "Unit Commitment and Operating Reserves with Probabilistic Wind Power Forecasts,” Trondheim, Norway, June 2011, with permission from IEEE.
} 
The chapter has the following structure: We provide a brief overview of the UC/ED model used in this chapter in Section 6.2. In Sections 6.3 and 6.4, we describe different approaches to probabilistic WPF and scenario reduction. Then, in Section 6.5 we compare the use of different forecasts and operational strategies in a case study of a test system with wind power and thermal generation. Finally, we summarize our findings, draw conclusions, and point out directions for future work.

\subsection{Scheduling of Energy and Operating Reserves}

We use the model first from the previous chapter to analyze the scheduling of energy and operating reserves in the electricity market. ${ }^{16}$ As a reminder, the objective of the UC model can be expressed as:

$$
\operatorname{Min} \sum_{S} \operatorname{prob}_{s} \cdot\left\{\sum_{t, i}\left[F C_{t, i}^{S}+C\left(R N S_{t}^{S}\right)+C\left(E N S_{t}^{S}\right)\right]\right\}+\sum_{t, i} S C_{t, i}
$$

The first component corresponds to minimizing the expected sum of fuel costs from thermal generators $(F C)$, the cost of unserved reserve $(C(R N S))$, and the cost of unserved energy $(C(E N S))$ over a set of scenarios $(s)$. The last part of the objective function is the thermal generator start-up costs $(S C)$. The problem is assumed to have an hourly time resolution and is solved for the next day. Two important constraints are to meet the demand for load and operating reserves $(O R)$ in all time periods $(t)$ and scenarios $(s)$ :

$$
\begin{aligned}
& \sum_{i} \operatorname{gen}_{\text {thermal }, i, t}^{S}+\operatorname{gen}_{\text {wind }, t}^{s}=\operatorname{load}_{t}-E N S_{t}^{s}, \quad \forall t, s \\
& \sum_{i} o r_{\text {thermal }, i, t}^{s} \geq O R_{\text {reg }, t}+O R_{\text {wind }, t}^{s}-R N S_{t}^{s}, \quad \forall t, s
\end{aligned}
$$

Both thermal generation and wind power contribute to meet the load, but we assume that only thermal generation can meet the operating reserve. We only consider one type of operating reserves: spinning reserves, which are provided by committed thermal units. ${ }^{17}$ However, we differentiate between reserve requirements to meet uncertainty in thermal generation and load $\left(O R_{r e g}\right)$ and wind power uncertainty $\left(O R_{\text {wind }}\right)$. If there are scenarios with scarcity where operating reserves or load are not being served, this is penalized in the objective function. The traditional UC constraints are also included, such as ramping constraints and minimum-up and -down constraints. Note that the model becomes deterministic simply by assuming that there is only one scenario.

\footnotetext{
${ }^{16}$ Some changes have been made to the model to improve the run-time. First, an improved representation of the binary commitment variables for thermal units has been implemented based on [92]. Second, the model is now implemented in AMPL and uses the state-of-the-art solver CPLEX for the resulting mixed integer linear (MIP) and linear (LP) programming problems.

${ }^{17}$ This assumption is obviously a simplification. For a discussion of different categories of operating reserve requirements, we refer to [76].
} 
In this analysis, we focus on the impact of wind power uncertainty. We assume that commitment decisions are made based on a DA wind power forecast. Then, we calculate the resulting RT dispatch with the realized wind power generation. ${ }^{18}$ The system's ability to handle RT fluctuation in wind power will depend on both the UC strategy and the operating reserve policy. UC schedules the generating units over the next day, taking inter-temporal constraints into account. Operating reserves impose additional constraints on unit availability for each individual hour. The wind power uncertainty can be accommodated by using a stochastic UC, which considers a range of scenarios for wind power generation for the next day. The wind power scenarios can be generated from probabilistic WPF. If the scenarios give a good representation of the wind power uncertainty, it should not be necessary to impose an additional operating reserve for wind, $O R_{\text {wind }}$. Alternatively, a deterministic UC model could be used to handle the wind power uncertainty through the additional reserve requirement. In this case, $O R_{\text {wind }}$ could be derived from probabilistic WPF. With the first approach, additional reserves are implicitly added through the stochastic UC, as opposed to the second approach, which uses an explicit reserve requirement for wind power. In either case, the performance of the scheduling decisions would depend on the quality of the probabilistic forecast. The different operating strategies are explored in the case study in Section 6.5.

\subsection{Probabilistic Wind Power Forecasts}

There are three main methods for estimating WPF uncertainty: (1) the Numerical Weather Prediction (NWP) point forecast approach, where the wind power uncertainty is directly computed from the NWP point forecast for weather; (2) the power output point forecast approach, which consists of forecasting uncertainty based on the historical WPF errors; and (3) the NWP ensemble approach, where NWP ensembles are used as input to the generation of probabilistic power forecasts. For a detailed discussion of state-of-the art in WPF and probabilistic forecasts, we refer to [1].

In our research, we are focusing on the statistical algorithms used in probabilistic WPF methods (1) and (2) above. A common approach is to use quantile regression (QR) to estimate the WPF uncertainty in terms of set of forecast quantiles [77]. In the project, we have developed novel statistical uncertainty forecasting approaches using kernel density forecasts (KDFs). Two new KDF-based WPF methods based on the Nadaraya-Watson (NW) and Quantile-Copula (QC) estimators are proposed in [93], [94], and [95].

KDF methods are based on conditional density estimation, which consists of estimating the density of a random variable, $Y$, knowing that the explanatory random variable, $X$, is equal to $x$. For the wind power problem, this consists of forecasting the wind power probability density function (pdf) at time step $t$ for each look-ahead time step $t+k$ of a given time-horizon when knowing a set of explanatory variables (e.g., NWP variables and measured wind power output). Mathematically, this set of conditions can be formulated as:

\footnotetext{
${ }^{18}$ Note that we do not consider the possibility of changing commitment decisions between DA scheduling and realtime dispatch. Intra-day commitment would clearly add flexibility to operational decisions. However, we focus on the impact of different UC and operating reserve strategies and do not consider the additional flexibility from intraday commitments.
} 


$$
\hat{f}_{P}\left(p_{t+k} \mid X=x_{t+k \mid t}\right)=\frac{f_{P, X}\left(p_{t+k}, x_{t+k \mid t}\right)}{f_{X}\left(x_{t+k \mid t}\right)}
$$

where $p_{t+k}$ is the wind power forecasted for look-ahead time $t+k, x_{t+k \mid t}$ are the explanatory variables forecasted for look-ahead time step $t+k$ and available at time step $t, f_{P, X}\left(p_{t+k}, x_{t+k \mid t}\right)$ is the multivariate density function, and $f_{X}\left(x_{t+k \mid t}\right)$ is the marginal density of $X$. The nonparametric conditional density estimation [96] for (6-4) is as follows:

$$
\hat{f}(p \mid X=x)=\sum_{i=1}^{N} K_{h_{p}}\left(p-P_{i}\right) \cdot \frac{K_{h_{x}}\left(x-X_{i}\right)}{\sum_{i=1}^{N} K_{h_{x}}\left(x-X_{i}\right)}
$$

where the kernel bandwidth $h_{p}$ controls the smoothness of each conditional density in the $p$ direction, while $h_{x}$ controls the smoothness between conditional densities in the $x$ direction.

The output is a pdf for wind power generation for each look-ahead time step of a specific time horizon. The pdf is general, from which several uncertainty representations can be derived (e.g., standard deviation, quantiles). For details on the implementation of the KDF WPF methods, we refer to [93], [94], and [95]. The forecasted pdfs for each look-ahead time step are the input of the scenario generation method described in Section 6.4. In the case study in Section 6.5, we compare the use of probabilistic forecasts from splines QR and KDF based on the NW estimator.

\subsection{Scenario Generation and Reduction}

The probabilistic forecasts described above express uncertainty information for specific points in time. However, in the UC problem, it is important to take inter-temporal relationships in the forecast uncertainty into account. This makes scenarios a more appropriate representation of the uncertainty. We use the WPF scenario generation approach described in [78] to convert quantiles or pdfs to forecast scenarios. In this approach, scenario sampling is performed using a Monte Carlo approach. A covariance matrix is estimated based on historical forecasting errors, and this is used to represent inter-temporal (hour-to-hour) correlations.

We can only use a limited number of scenarios as input to the stochastic UC model because of the computational complexity of the problem. In most cases, there will be a need for scenario reduction. In this chapter, we are testing different methods to generate a reduced set of scenarios. One simple approach is to select a random subset of the original scenarios. In the case study below, this approach is referred to as scenario reduction method 1 (SR1). Within the power systems domain, it is common to use the scenario reduction method from [89], which we refer to as SR2. Here, a reduced scenario set is derived from the Kantorovich distance between the original and reduced set of scenarios, taking scenario probabilities and distances of scenario values into account. 
We are also exploring alternative approaches to generate a reduced set of representative scenarios, as outlined in Chapter 5. The idea in Chapter 5 and in [97] is to find the most attractive scenario that has a higher number of neighbors according to a flexible distance metric, which is selected to be compatible with the problem to be solved. For the stochastic UC problem, we use the maximum absolute deviation distance:

$$
d(i, j)=\max \left\|X_{t}^{(i)}-X_{t}^{(j)}\right\|, \quad t=1, \ldots, T
$$

where $X^{(i)}$ and $X^{(j)}$ are the two scenarios being compared, and $T$ is their dimension. The two scenarios are considered to be "neighbors" if the distance between them is lower than the admissible tolerance, that is, $d(i, j) \leq \varepsilon$. When the more attractive scenario and all its "neighbors" are found, they are cut from the initial set of scenarios, thereby forming a cluster. The procedure is repeated until the attractive scenario cannot find a neighbor within the tolerance. The remaining scenarios without a neighbor are considered outliers. Starting from a small tolerance value, the total number of clusters may be higher than the desired number of scenarios set for the stochastic problem. The algorithm is then repeated with a lower $\varepsilon$ in order to find the admissible tolerance that provides the desired number of clusters. Finally, each cluster is represented by its more representative scenario. Here, the scenario presenting the lower sum of distances, according to (6-6), with respect to all other members of the cluster has been used. The probability associated with each representative scenario is given by the sum of the probabilities of its cluster components. We refer to this method as SR3.

\subsection{Case Study}

The purpose of the case study is to test the different probabilistic WPF approaches, scenario reduction methods, and operating strategies on DA UC decisions and the resulting RT ED. Thermal units are committed based on a DA wind power forecast by using stochastic or deterministic UC. The RT ED is then solved with the realized availability of wind power. We focus solely on the impact of WPF uncertainty. Other uncertainties, like generator outages and load forecast errors, are therefore omitted. We first discuss the quality of the probabilistic wind power forecasts and resulting wind power scenarios. Then, we examine the impact on UC and ED results in a simple case study with wind power and 10 thermal units.

\subsubsection{Probabilistic Wind Power Forecasts}

Time series of the DA deterministic point forecasts and realized wind power output for 15 hypothetical locations in Illinois in 2006 were aggregated and used as the starting point for the probabilistic forecasts. The data were obtained from the Eastern Wind Integration and Transmission Study (EWITS) [83].

We use both QR and NW KDF to generate probabilistic wind power forecasts. The wind power point forecast is used as the only explanatory variable in both cases. The period from January to July is used to train the two statistical models, whereas the resulting forecast data for October to December are used in the analysis. An example of a quantile forecast is shown in Fig. 6-1.

In order to evaluate the outputs from the two different forecast uncertainty estimation procedures, we focused on the calibration forecasts. Calibration is a measure of how well the forecasted quantiles match the observed values [1]. Fig. 6-2 depicts the calibration diagram for 
the QR and NW methods. There is clearly a quite significant negative deviation (corresponding to an over-forecast) for both methods, with QR having the largest deviation. ${ }^{19}$ We provide a more detailed comparison of different probabilistic WPF methods in [93], [94], and [95]. The results indicate that KDF forecasts outperform QR in terms of calibration, whereas QR tends to perform better in terms of sharpness, which is a measure of the width of the forecast distribution.

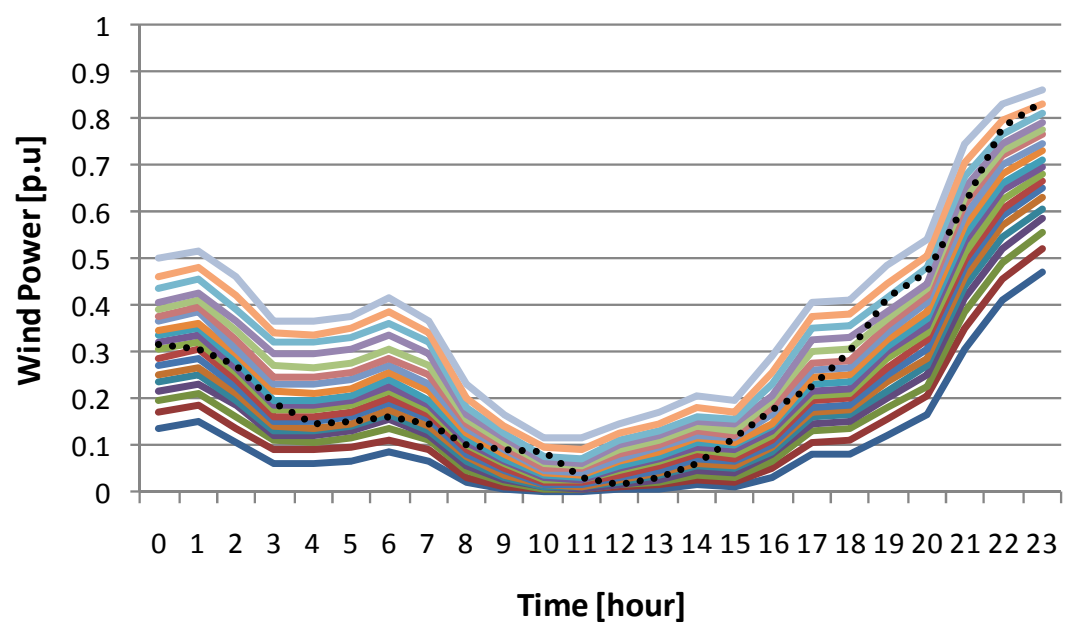

Fig. 6-1 Probabilistic forecasts of wind power quantiles $(5 \%, 10 \%, \ldots 95 \%)$ based on $Q R$ and realized wind power generation (dotted line) for day 1.

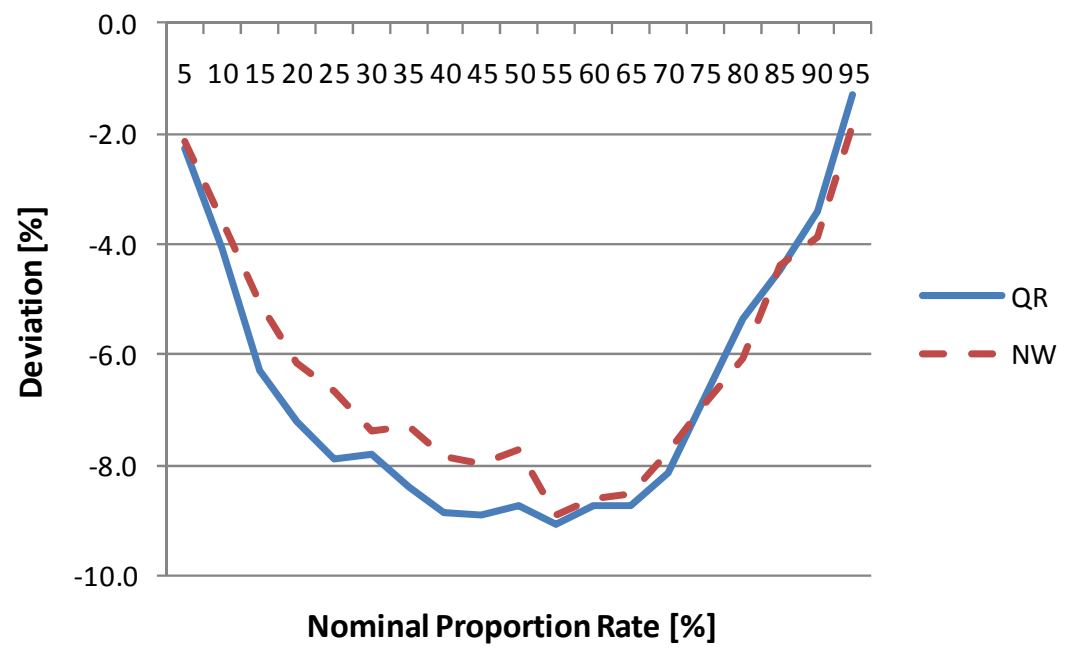

Fig. 6-2 Calibration diagram for QR and NW methods, Oct.-Dec. 2006.

Based on the probabilistic QR and NW forecasts, we generated 1,000 scenarios with the sampling method described in [78]. Scenario reduction is then used to reduce the full set to either 10 or 100 scenarios by using the three scenario reduction methods described in Section 6.4. For SR2, we use the implementation in GAMS/SCENRED [98] with standard parameters. The SR3 method is implemented in Matlab.

\footnotetext{
${ }^{19}$ The large deviations may have to do with the short evaluation period.
} 
Fig. 6-3 shows that the averages of the reduced scenarios are close to the average of the original 1,000 scenarios, although some deviations can be seen with random selection (SR1) and for the clustering-based scenario reduction method (SR3). Results in Fig. 6-4 and Fig. 6-5 show that the reduced scenarios based on SR2 and SR3 tend to have a significantly lower variance than the original 1,000 scenarios. The variance of the randomly selected scenarios (SR1) is higher on average than for SR2 and SR3, and with 100 scenarios, this method yields a closer match to the variance of the original scenarios.

Table 6-1 shows a comparison of the means and variances for the original and reduced scenarios. The statistics are calculated from the hourly forecasted scenario data over the full 3-month period. All of the reduced sets are close to the original scenarios in terms of the overall average over the period. However, SR2 has a lower mean absolute error (MAE) than the other two methods. When it comes to variance, SR1 provides a closer fit than SR2 and SR3 do, both in terms of average and MAE. It is evident that both SR2 and SR3 result in a significant reduction in the scenario variance compared to the original scenario set. Still, the fit improves by increasing the size of the reduced scenario set from 10 to 100 .

Further analysis of statistical properties could be performed by analyzing additional moments of the scenario distributions. A comparison of scenario reduction [89] and moment matching [99] approaches for scenario tree construction is conducted in [100]. The authors found that the moment-matching approach yields a better fit compared to the original scenario set in terms of mean, variance, skewness, and kurtosis. However, the scenario reduction provides better correspondence in terms of autocorrelation. Scenario reduction is also considerably faster computationally than is moment matching. The authors also tested the two approaches as input to a scheduling model for the electricity market within a region of Germany and found that scenario trees derived from moment matching yields the lowest operational cost [100].

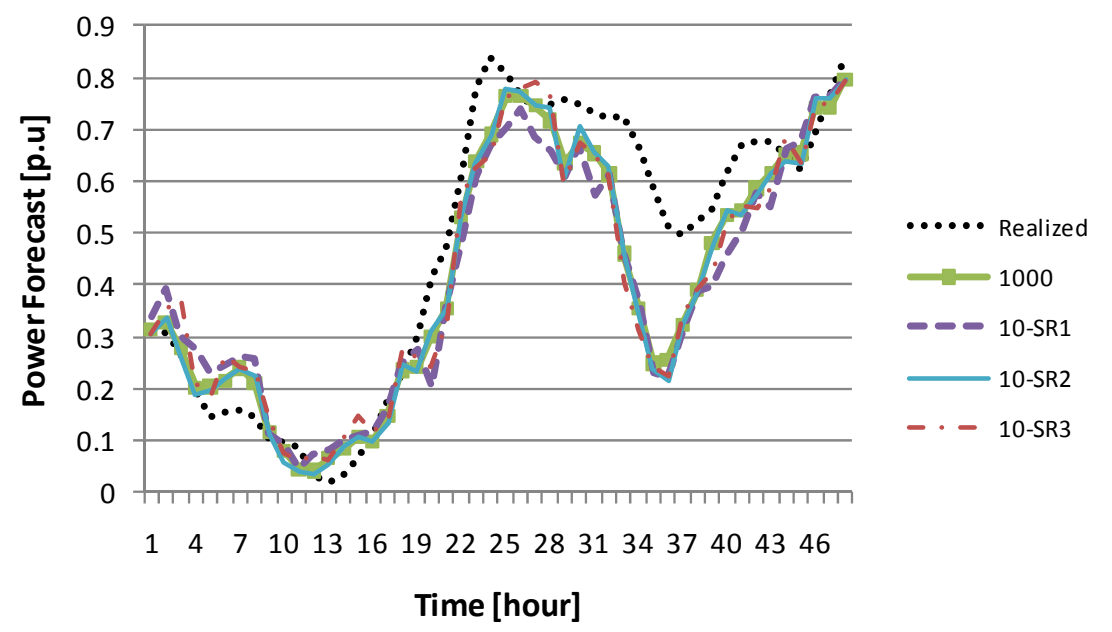

Fig. 6-3 Averages of 10 scenarios with reduction methods SR1, SR2, and SR3 as compared to the average of the original 1,000 scenarios, QR, days 1 and 2. 


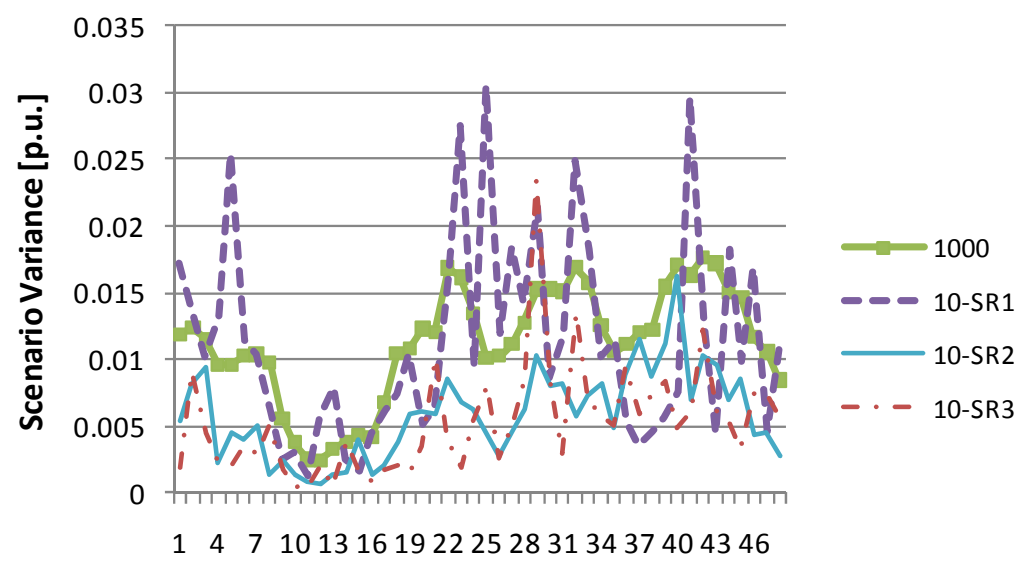

Time [hour]

Fig. 6-4 Variances for 10 scenarios with reduction methods SR1, SR2, and SR3 as compared to the average of the original 1,000 scenarios, QR, days 1 and 2.

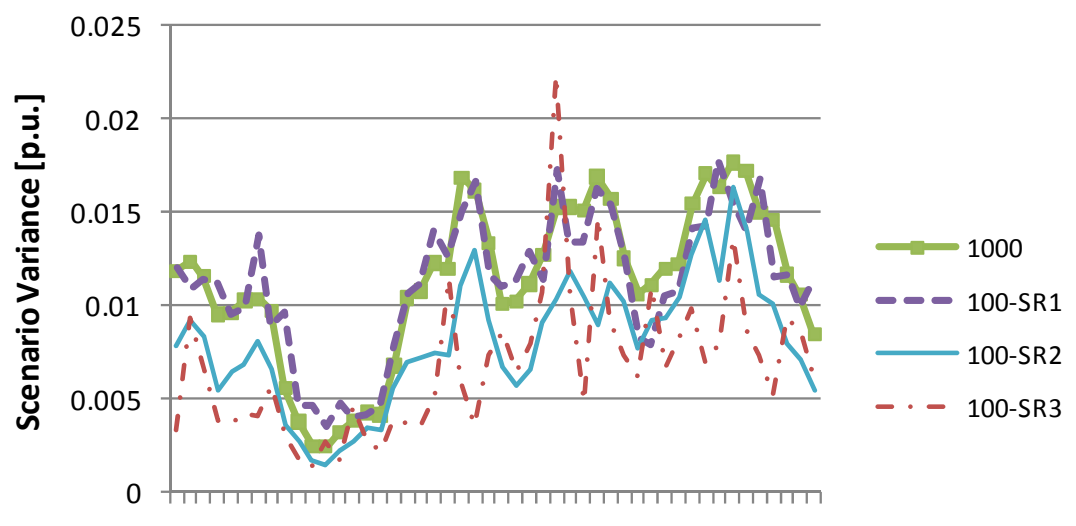

14710131619222528313437404346

Time [hour]

Fig. 6-5 Variances for 100 scenarios with reduction methods SR1, SR2, and SR3 as compared to the average of the original 1,000 scenarios, QR, days 1 and 2.

Table 6-1 Summary statistics for scenarios (QR) with different reduction methods: average (AVG), mean absolute error (MAE), Oct.-Dec.

\begin{tabular}{|c|c|c|c|c|c|c|c|}
\hline & 1,000 & $\begin{array}{c}10- \\
\text { SR1 }\end{array}$ & $\begin{array}{c}10- \\
\text { SR2 }\end{array}$ & $\begin{array}{c}10- \\
\text { SR3 }\end{array}$ & $\begin{array}{c}100- \\
\text { SR1 }\end{array}$ & $\begin{array}{c}100- \\
\text { SR2 }\end{array}$ & $\begin{array}{c}100- \\
\text { SR3 }\end{array}$ \\
\hline $\begin{array}{c}\text { Mean } \\
\text { (AVG) }\end{array}$ & 0.419 & 0.417 & 0.418 & 0.417 & 0.418 & 0.418 & 0.417 \\
\hline $\begin{array}{c}\text { Mean } \\
\text { (MAE) }\end{array}$ & 0 & 0.026 & 0.010 & 0.024 & 0.008 & 0.004 & 0.021 \\
\hline $\begin{array}{c}\text { Variance } \\
\text { (AVG) }\end{array}$ & 0.011 & 0.011 & 0.006 & 0.005 & 0.011 & 0.008 & 0.007 \\
\hline $\begin{array}{c}\text { Variance } \\
\text { (MAE) }\end{array}$ & 0 & 0.0041 & 0.0055 & 0.0060 & 0.0012 & 0.0027 & 0.0042 \\
\hline
\end{tabular}




\subsubsection{Overview of Simulated Cases}

We use the same assumption for thermal generators, loads, and realized wind power availability as in [86]. The system consists of ten thermal generators with a mix of base, intermediate, and peak units. The characteristics of the units are the same as in Chapter 4 (Table 4-1). A time series with 91 days (October-December) of data for loads and wind power were simulated. The load data are based on scaled historical load profiles for Illinois from 2006 and therefore are synchronized with the wind power data. The total installed capacity is 1,662 MW of thermal power and $500 \mathrm{MW}$ of wind power, and the peak load is 1,500 MW. The total wind energy amounts to $20 \%$ of the load within the 91-day simulation period. The costs of unserved reserve and load are assumed to be $\$ 1,100 / \mathrm{MWh}$ and $\$ 3,500 / \mathrm{MWh}$, respectively. These costs are included in the objective function of the UC problem, as shown in (6-1).

An overview of the simulated cases is provided in Table 6-2 and Table 6-3. The cases differ in terms of which UC strategy, operating reserve requirements, wind power forecast, and scenario reduction method is employed. For the deterministic cases (Table 6-2), the first case uses a perfect forecast and therefore serves as a benchmark for the analysis. The PF-QR and PF-NW cases use deterministic forecasts (i.e., the 50\% quantile from either the QR or NW forecasts), without additional operating reserve for wind uncertainty, $O R_{\text {wind }}$. In RF-QR and RF-NW, we add a fixed $O R_{\text {wind }}$ equal to the average difference of the $50 \%$ and $5 \%$ WPF quantiles. Finally, in the RD-QR and RD-NW cases, $O R_{\text {wind }}$ is dynamic, updated each day, and set equal to the difference between the $50 \%$ and $5 \%$ quantiles. Because the $50 \%$ quantile is used as the point forecast, the demands for energy and regular operating reserves should be met with a probability of 0.95 . The optimal choice of quantile range for $O R_{\text {wind }}$ generally depends on the cost of scheduling additional reserves compared to the cost of not meeting the regular reserve requirements. It should be noted that a more rigorous approach could be used to derive $O R_{\text {wind }}$ (e.g., based on the ideas in [75]).

For the stochastic cases, we vary the probabilistic WPF approach, the scenario reduction method, and the number of scenarios, as shown in Table 6-3. $O R_{\text {wind }}$ is set to zero for the stochastic cases, with the assumption that the stochastic UC strategy internally addresses the wind power uncertainty.

Table 6-2 Simulated cases with deterministic UC.

\begin{tabular}{|c|c|c|}
\hline Case & Additional Reserve: OR $_{\text {wind, }}{ }^{*}$ & Forecast \\
\hline PERF & None & Perfect \\
\hline PF-QR & None & QR: $50 \%$ quantile \\
\hline PF-NW & None & NW: $50 \%$ quantile \\
\hline RF-QR & Fixed: avg. 50-5\% quantile & QR: $50 \%$ quantile \\
\hline RF-NW & Fixed: avg. 50-5\% quantile & NW: $50 \%$ quantile \\
\hline RD-QR & Dynamic: $50-5 \%$ quantile & QR: $50 \%$ quantile \\
\hline RD-NW & Dynamic: $50-5 \%$ quantile & NW: $50 \%$ quantile \\
\hline
\end{tabular}

* This reserve is applied at the UC stage only. All cases use a regular reserve, $O R_{\text {reg,t }}$, equal to $10 \%$ of hourly loads in both UC and ED. 
Table 6-3 Simulated cases with stochastic UC.

\begin{tabular}{|c|c|c|c|}
\hline Case & Forecast & Scenario Reduction* & No. of Scenarios \\
\hline S10-QR1 & QR & SR1 & 10 \\
\hline S10-QR2 & QR & SR2 & 10 \\
\hline S10-NW1 & NW & SR1 & 10 \\
\hline S10-NW2 & NW & SR2 & 10 \\
\hline S100-QR1 & QR & SR1 & 100 \\
\hline S100-QR2 & QR & SR2 & 100 \\
\hline S100-QR3 & QR & SR3 & 100 \\
\hline
\end{tabular}

$* \mathrm{SR} 1=$ random, $\mathrm{SR} 2=$ traditional scenario reduction, and SR3 = scenario clustering.

\subsection{UC and ED Results}

The total operating costs for the different cases over the 91-day period are shown in Fig. 6-6 and Fig. 6-7. Additional results are summarized in Tables 6-4 and 6-5.

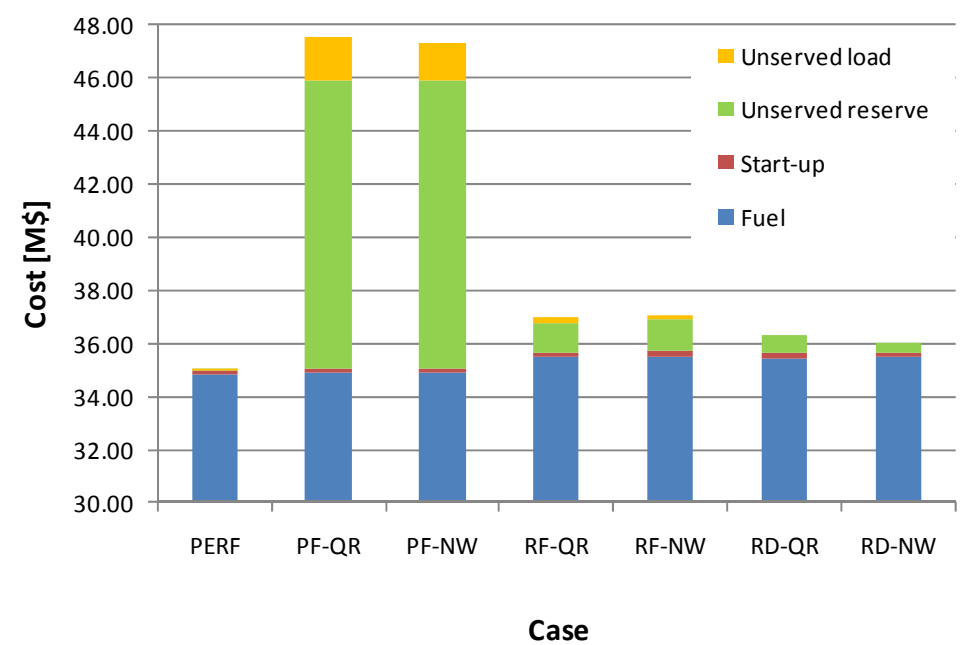

Fig. 6-6 Overview of simulated total costs for deterministic cases.

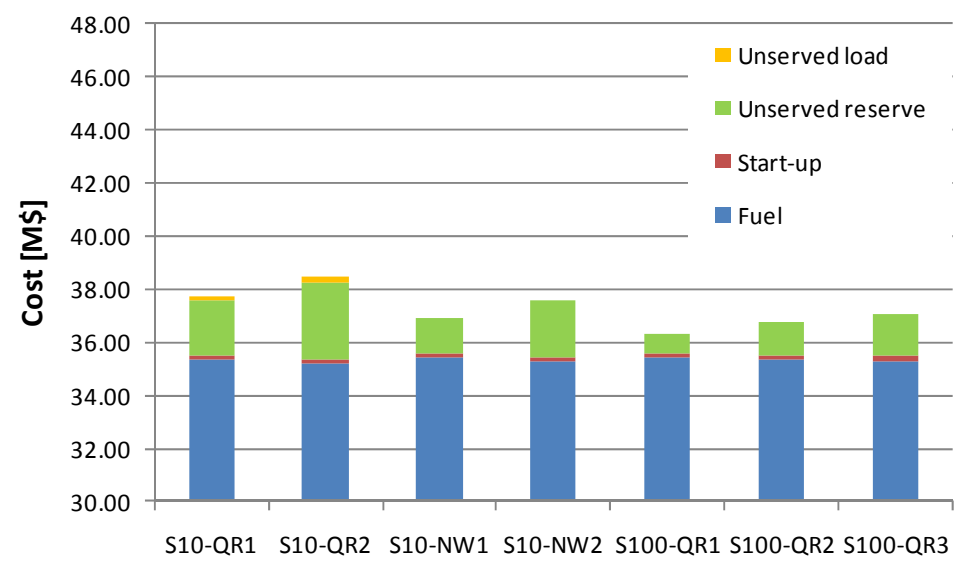

Case

Fig. 6-7 Overview of simulated total costs for stochastic cases. 
Table 6-4 Summary of other results: deterministic cases.

\begin{tabular}{|c|c|c|c|c|}
\hline Case & $\begin{array}{c}\text { No. of } \\
\text { Start-ups }\end{array}$ & $\begin{array}{c}\text { Load } \\
\text { Curtailment } \\
\text { [hours] }\end{array}$ & $\begin{array}{c}\text { Reserve } \\
\text { Curtailment } \\
\text { [hours] }\end{array}$ & $\begin{array}{c}\text { Avg. } \\
\text { Reserve } \\
{[\mathrm{MW}]}\end{array}$ \\
\hline PERF & 225 & 0 & 7 & 155.2 \\
\hline PF-QR & 228 & 14 & 268 & 153.2 \\
\hline PF-NW & 231 & 12 & 273 & 152.2 \\
\hline RF-QR & 280 & 1 & 38 & 174.5 \\
\hline RF-NW & 287 & 1 & 40 & 174.6 \\
\hline RD-QR & 267 & 0 & 31 & 170.8 \\
\hline RD-NW & 266 & 0 & 22 & 173.8 \\
\hline
\end{tabular}

Table 6-5 Summary of other results: stochastic cases.

\begin{tabular}{|c|c|c|c|c|}
\hline Case & $\begin{array}{c}\text { No. of } \\
\text { Start-ups }\end{array}$ & $\begin{array}{c}\text { Load } \\
\text { Curtailment } \\
\text { [hours] }\end{array}$ & $\begin{array}{c}\text { Reserve } \\
\text { Curtailment } \\
\text { [hours] }\end{array}$ & $\begin{array}{c}\text { Avg. } \\
\text { Reserve } \\
{[\mathrm{MW}]}\end{array}$ \\
\hline S10-QR1 & 278 & 1 & 71 & 169 \\
\hline S10-QR2 & 282 & 1 & 101 & 164 \\
\hline S10-NW1 & 267 & 0 & 49 & 171 \\
\hline S10-NW2 & 252 & 0 & 79 & 168 \\
\hline S100-QR1 & 234 & 0 & 35 & 175 \\
\hline S100-QR2 & 235 & 0 & 50 & 171 \\
\hline S100-QR3 & 234 & 0 & 68 & 170 \\
\hline
\end{tabular}

The performance of the system clearly depends on the UC and operating reserve strategy and on the WPF approach. We make the following main observations:

- Using a deterministic point forecast without additional reserve is too risky and results in high levels of unserved load and reserve.

- For deterministic cases, a dynamic reserve requirement based on the probabilistic forecast performs better than a fixed additional reserve.

- A dynamic reserve based on a NW forecast performs better than a dynamic reserve based on QR forecast, possibly caused by the better calibration of the NW forecast (Fig. 6-2).

- With stochastic UC, scenarios based on NW forecasts perform better than scenarios from QR.

- Formal scenario reduction based on SR2 and SR3 does not improve the overall system performance under a stochastic UC strategy compared to a random selection of scenarios (SR1). This may have to do with the decrease in scenario variance under SR2 and SR3 (Table 6-1), which may result in less hedging and more risky scheduling.

- Increasing the number of scenarios improves the performance of the stochastic UC strategy. However, the computational burden increases. In these cases, the run-time with 100 instead of 10 scenarios is 15 to 20 times longer.

- Some additional reserve is needed also in stochastic UC to cover wind power uncertainty not captured by the probabilistic forecasts and the limited number of scenarios. The best stochastic case (S100-QR1) yields a slightly higher total cost than that of the best deterministic case (RD-NW).

- The two best cases (S100-R1 and RD-NW) both make use of probabilistic WPF information. 


\subsection{Current Work: IL Case Study}

In our recent work [101], we are scaling up the UC modeling framework to handle larger systems in a more realistic manner. In particular, we are testing different UC models and operating reserve strategies on two-settlement electricity markets to explore the potential use of probabilistic WPF to help power system operators' scheduling and dispatch decisions. We are using the power system in Illinois as a test bed to analyze the implications of a large-scale expansion of wind power in a real-world electricity market.

The Illinois system consists of thermal generation and wind power, with only a negligible amount of hydro generation. In the case study, loads and thermal generation corresponds to the situation in Illinois in 2006, whereas we assume that additional wind power is added to the system to represent a future scenario with a high penetration of wind power. The Illinois system in 2006 consisted of 210 thermal plants with a total capacity of 41,380 MW. We assume that the total installed capacity of wind power in the system is 14,000 MW. The wind power generation fluctuates between 0 and 12,680 MW. Under this assumption, wind power provides $20 \%$ of the in-state load and $16.6 \%$ of the total load including export in the July-October period. The hourly load profile is based on historical data from two large utilities in the state of Illinois for the months of July to October of 2006.

We simulate the main steps in the operation of two-settlement electricity markets with focus on the use of wind power forecasts (Fig. 6-8). The commitment schedule for thermal units is first determined based on a DA wind power point forecast in the DA market, using deterministic UC. Then, we simulate the reliability assessment commitment (RAC), where the commitment of faststarting units is adjusted based on a 4-hour-ahead probabilistic forecast, using stochastic or deterministic UC. The RT ED is then solved with the realized availability of wind power. We focus solely on the impact of WPF uncertainty. Other uncertainties, like generator outages and load forecast errors, are therefore omitted, similar to the 10-unit case study presented above. Note that the analysis of the 10-unit case above and in Chapter 4 does not consider the RAC, as all commitment decisions are made at the DA stage. The simulation set-up for the Illinois study is therefore better aligned with current operational practices, as discussed in Chapter 3.

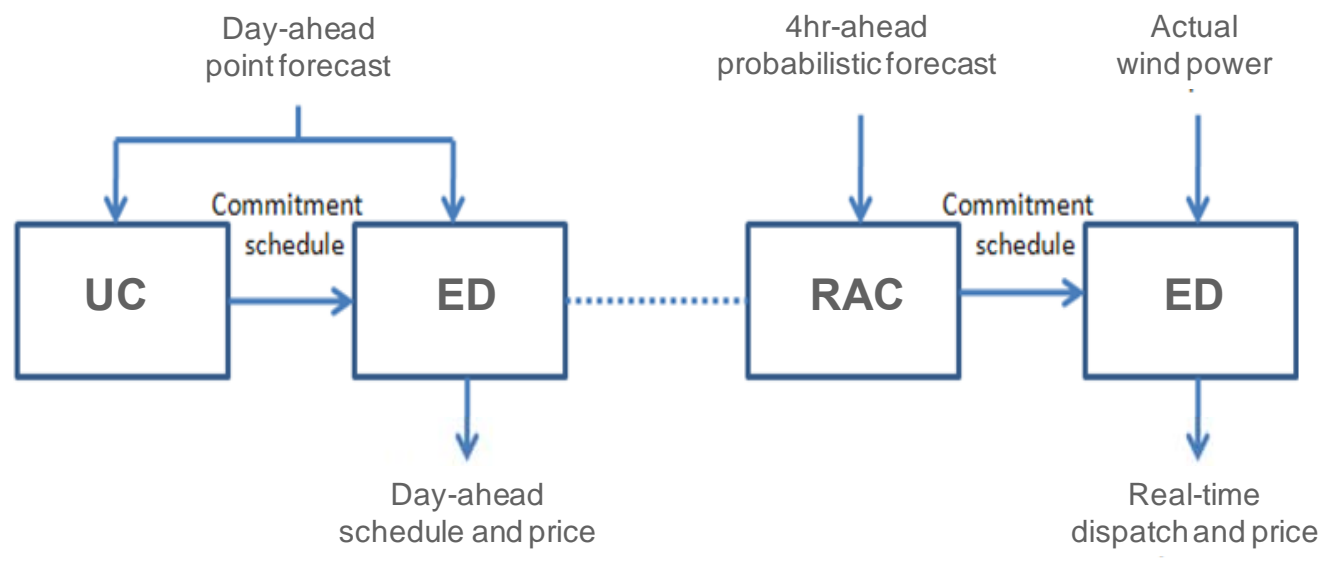

Fig. 6-8 Simulation of two settlement electricity markets in Illinois case study. 
The results from the Illinois case study show that the re-commitment decisions at the RAC stage are very important for the cost and reliability of the system. We find that additional operating reserves are required to handle the uncertainty in wind power. When basing the re-commitment decisions on a deterministic UC model, the use of dynamic operating reserves derived from the probabilistic forecast gives better performance than fixed reserve requirements, as the operating reserve levels are better aligned with the wind power forecast uncertainty. A stochastic UC model with wind power scenarios leads to different re-commitment decisions and RT dispatch. However, dynamic operating reserves and stochastic UC give similar results in terms of total system cost. Hence, from a qualitative perspective, the results from the Illinois case study are in line with the 10-unit results presented earlier in this chapter, although a more comprehensive modeling framework is applied. The detailed results from the Illinois case also illustrate that inaccurate wind power forecasts can lead to over- and under-commitment of generation capacity and therefore have large implications for system reliability and RT prices. Further details on the Illinois case study are provided in [101].

\subsection{Conclusion}

The results of the analysis in this chapter clearly illustrate the potential value of probabilistic WPF in power system operations. Such forecasts can be incorporated into a deterministic UC through probabilistic reserve requirements or can provide scenarios as input to a stochastic UC. Some advantages of the deterministic approach with dynamic reserves includes that it is better aligned with current operating procedures. Furthermore, from a computational perspective, it is a simpler model with less computational burden. However, this approach does not capture the effect of inter-temporal ramping events. It also does not consider uncertainty and its cost in the objective function. In contrast, stochastic UC does address inter-temporal variability through the scenario representation of uncertainty. Moreover, the total cost, including the expected cost of scarcity, is explicitly taken into account in the objective function. However, the switch to stochastic UC involves a more radical departure from current practice; furthermore, it may run into computational constraints in large systems, and the benefits in terms of cost savings may be limited.

More work is needed to improve the quality of probabilistic WPF, both in terms of pdf estimation and generation of adequate scenarios. However, there will always be inaccuracy in probabilistic forecasts, just as in point forecasts. This calls into question the overall risk paradigm under which scheduling decisions are made. In stochastic UC, with the minimization of expected cost, one could adjust the risk level by changing the costs of unserved energy and reserves in the objective function. However, it may also be required to consider other approaches based on different decision paradigms, such as utility theory, value at risk, robust optimization, or regret. The optimal decision strategy does not only depend on the risk preferences of the system operator, but also on the quality of the probabilistic forecast. 
This page intentionally blank. 


\section{WIND POWER TRADING UNDER UNCERTAINTY IN ELECTRICITY MARKETS ${ }^{20}$}

This chapter presents a new model for optimal trading of wind power in DA electricity markets under uncertainty in wind power and prices. The model considers settlement mechanisms in markets with locational marginal prices (LMPs), where wind power is not necessarily penalized from deviations between DA schedule and RT dispatch. We use quantile regression and kernel density estimation to produce a probabilistic wind power forecast, whereas uncertainties in DA and RT prices are assumed to follow a normal distribution. Utility theory and conditional value at risk (CVAR) are used to represent the risk preferences of the wind power producers. The model is tested on data from a hypothetical wind farm located in the state of Illinois and combined with historical LMP data. The results show that the model can effectively control the trade-off between risk and return for wind power producers operating in volatile electricity markets.

The following nomenclature is used in this chapter:

$\begin{array}{ll}\pi_{h}^{m} & \text { energy market profit, hour } h(h=1 \ldots 24), \text { scenario } m(m=1 \ldots M)[\mathrm{US} \$] \\ q_{D A, h} & \text { quantity bid into DA market, hour } h[\mathrm{MW}] \\ q_{R T, h}^{m} & \text { quantity bid into RT market, hour } h[\mathrm{MW}] \\ \bar{q}_{d, h}^{m} & \text { max. potential quantity delivered, hour } h, \text { scenario } m[\mathrm{MW}] \\ q_{d, h}^{m} & \text { quantity delivered, hour } h \text {, scenario } m[\mathrm{MW}] \\ p_{D A, h}^{m} & \text { projected DA LMP, hour } h, \text { scenario } m[\mathrm{US} \$ / \mathrm{MWh}] \\ p_{R T, h}^{m} & \text { projected RT LMP, hour } h, \text { scenario } m[\mathrm{US} \$ / \mathrm{MWh}] \\ \mu_{D A, h}, \mu_{R T, h} & \text { mean DA, RT prices, hour } h, \text { scenario } m[\mathrm{US} \$ / \mathrm{MWh}] \\ \sigma_{D A, h}, \sigma_{R T, h} & \text { stdev DA, RT prices, hour } h, \text { scenario } m[\mathrm{US} \$ / \mathrm{MWh}]^{2} \\ \rho_{h} & \text { DA-RT price corr., hour } h, \text { scenario } m[\mathrm{US} \$ / \mathrm{MWh}]^{2} \\ \varepsilon_{D A}^{m}, \varepsilon_{R T}^{m} & \text { Random sample, DA, RT prices, scenario } m[\mathrm{Norm} \sim(0,1)] \\ p^{m} o b_{m} & \text { probability of scenario } m \\ p e n_{D A} & \text { penalty for deviation between DA schedule and RT delivery } \\ U_{h}^{m}\left(\pi_{h}^{m}\right) & {[\mathrm{US} \$ / \mathrm{MWh}]} \\ \beta & \text { utility, hour } h, \text { scenario } m \\ C V A R_{h} & \text { risk parameter in utility function } \\ t h & \text { conditional value at risk, hour } h \\ w & \text { threshold for CVAR } \\ \pi_{h}^{q u a n t, t h} & \text { weight assigned to CVAR } \\ & \text { quantile } t h \text { of profit distribution, hour } h \\ & \end{array}$

\footnotetext{
${ }^{20}$ The original ideas behind the model described in this chapter were first presented in [24]. Our most recent results from applying the model on a real-world wind farm located in the Midwest are presented in [102].
} 


\subsection{Introduction}

There has been a rapid expansion of wind energy in recent years, with the global installed capacity of wind power exceeding $200 \mathrm{GW}$ [3]. Wind power is expected to continue its growth in many countries, including the United States [44]. For wind farm owners, it is therefore becoming increasingly important to manage the risk of participating in the electricity market. In the United States, most of the wind power is sold under long-term power purchasing agreements (PPAs). The PPAs lock in the price of delivered electricity and thereby stabilize the revenue stream [103]. Nevertheless, a significant share of wind power is sold directly to the electricity market. In 2009, 38\% of the new installed wind power capacity was sold as merchant generation, and most of this energy was traded in regions with open access electricity markets [103]. For merchant wind generation, it is very important to apply scheduling and trading strategies that reflect the uncertainty in wind power and prices and the corresponding trade-off between risk and return for the wind farm owner. At the same time, purchasers of PPAs will have to schedule the contracted wind power into the electricity market, and consider how wind power influences the scheduling strategy of the rest of their portfolio.

In this chapter, we discuss scheduling and trading of wind power with focus on DA electricity markets. We first provide an overview of existing literature on this topic. We then discuss the trading of wind power in U.S. markets with LMPs, including settlement procedures and their influence on trading decisions. We present an optimization model, which finds the optimal wind power bid under uncertainty in forecasts for wind power, as well as DA and RT prices. Finally, we apply the model in a case study of a hypothetical wind farm in the state of Illinois. We analyze how DA bids depend on risk preferences and deviation penalty schemes, under the assumption that wind power is sold as merchant generation.

\subsection{Literature Review}

Trading of wind power in electricity markets is still a relatively new problem. A limited number of methods have been proposed in the literature to address the problem, as outlined below. Bathurst et al. [104] use a simple wind power uncertainty representation based on Markov probabilities and show how this approach can be used to reduce imbalance costs from short-term wind power trading in the UK market. Bremnes [23] demonstrates how probabilistic wind power forecasts can be created by means of local quantile regression. He shows that if imbalance costs are deterministic, the profit maximizing bid is equal to the $\mathrm{c}_{+} /\left(\mathrm{c}_{+}+\mathrm{c}_{-}\right)$wind power forecast quantile, where $c_{+}$and $c_{-}$are the imbalance costs of excess and shortage, respectively. Matevosyan and Söder [19] use an ARIMA model for the wind speed and a power curve to produce wind power forecast scenarios. The authors propose a stochastic optimization model for DA trading that considers a more complex imbalance cost scheme akin to the rules in the Scandinavian electricity market (Nord Pool). Pinson et al. [17] also focuses on minimizing the imbalance cost. In a case study, the authors demonstrate the advantages of using probabilistic wind power forecasts to derive DA trading strategies similar to the one proposed in [23]. Bourry et al. [20] use probabilistic wind power forecasts based on kernel density estimation (KDE) to estimate a probability distribution for the imbalance cost. A risk-based decision approach based on conditional value at risk (CVAR) is used to derive the optimal bid under the resulting uncertainty in imbalance cost. Usaola and Moreno [105] focus on bidding of wind energy in intra-day markets. This is framed as a profit-maximization problem with simple representations of uncertainties in wind power predictions and prices (intra-day and balancing). Morales et al. 
[106] propose a stochastic programming model for wind power trading in multiple consecutive markets. Uncertainty in wind power and prices are represented as seasonal ARIMA models. However, the probabilistic models are general and are not linked to forecast information. The authors derive optimal offer curves (bid-quantity) using CVAR as a measure for risk preferences. Finally, Dent et al. [107] derive analytical wind power bidding strategies under the assumption of a continuous probability function for wind power. The authors also consider situations where the RT price is a function of wind generation and use CVAR to represent risk-averse trading behavior.

The main features of several of the wind power models outlined above are summarized in Table 7-1, with a comparison to the model described in this chapter.

The literature summarized above focus on the conditions in European electricity markets. In general, these markets have different prices for positive and negative imbalances in RT [105]. Although there are regional differences in market rules within Europe, wind power producers are therefore generally penalized if their deviation is against the system balance. In contrast, our analysis adds new insights by focusing on typical settlement procedures in U.S. electricity markets with LMPs. In these markets, there is only one RT price. Hence, a wind power producer may actually benefit from deviating from its advance schedule, depending on the difference in DA and RT LMPs. We emphasize the use of probabilistic WPF methods to support trading decisions, and we use different methods to produce probabilistic forecasts. We also consider uncertainties in both DA and RT prices and their impacts on the risk faced by wind power producers operating in LMP markets. Moreover, we consider different approaches to represent risk preferences, either through the expected value (i.e., risk neutral), a CVAR measure (i.e., trade-off between expected value and risk), or with a classical utility function. As far as we

know, utility theory has not been used in previous literature on wind power trading. Finally, our model considers the impact of market design on the optimal trading decision. In particular, we analyze how a deviation penalty would influence the optimal DA bidding decision.

\subsection{Wind Power in LMP Markets}

Most U.S. electricity markets are operated ISO/RTOs. In most cases, ISO/RTOs use LMPs and the so-called two-settlement system for market clearing, as briefly outlined in Chapter 3 . This means that the DA schedule is settled at the DA LMP, and that any deviation between the DA schedule and RT delivery is settled at the RT LMP [108]. In most cases, there are no intra-day markets between DA and RT in the United States.

Wind power producers typically have the option to participate in the DA market. In general, there may be some charges for deviations between the RT delivery and the DA schedule. So far, wind power has typically been exempt from imbalance charges in most U.S. markets [26][63]. However, this situation may change as several ISO/RTOs are revising their current rules [109]. The Federal Energy Regulatory Commission (FERC) is also revising wholesale market rules for better integration of variable generation [110]. The handling of wind power in general and particularly the use WPF in U.S. markets were discussed in more detail in Chapter 3. 
Table 7-1 Overview of published research/models for wind power trading.

\begin{tabular}{|c|c|c|c|c|c|c|c|}
\hline Model & WPF & Price Forecast & $\begin{array}{l}\text { Objective/ } \\
\text { Risk }\end{array}$ & $\begin{array}{l}\text { Uncertainty } \\
\text { Representation }\end{array}$ & Market Rules & $\begin{array}{l}\text { Intraday } \\
\text { Trading }\end{array}$ & $\begin{array}{l}\text { Multi-Day } \\
\text { Simulator }\end{array}$ \\
\hline $\begin{array}{l}\text { Bathurst } \\
\text { et al. } 2002[104]\end{array}$ & $\begin{array}{l}\text { Persistence } \\
\text { w/prob. table }\end{array}$ & $\begin{array}{l}\text { No, fixed bal. } \\
\text { price relations }\end{array}$ & $\begin{array}{l}\text { Min. (max.) } \\
\text { imbalance cost }\end{array}$ & $\begin{array}{l}\text { Probability table } \\
\text { for wind power }\end{array}$ & $\begin{array}{l}\text { UK (NETA), } \\
\text { regulation up/dn, } \\
\text { short term }\end{array}$ & $\begin{array}{l}\text { Yes, but no DA } \\
\text { trading }\end{array}$ & $\begin{array}{l}\text { Yes, analysis of } \\
\text { one year }\end{array}$ \\
\hline $\begin{array}{l}\text { Matevosyan } \\
\text { and Söder } 2006 \\
{[19]}\end{array}$ & $\begin{array}{l}\text { Wind speed } \\
\text { forecast } \mathrm{w} / \text { power } \\
\text { curve }\end{array}$ & $\begin{array}{l}\text { Imbalance price } \\
\text { scenarios from } \\
\text { last days }\end{array}$ & $\begin{array}{l}\text { Min. imbalance } \\
\text { cost }\end{array}$ & $\begin{array}{l}\text { WP: ARMA from } \\
\text { errors }\end{array}$ & $\begin{array}{l}\text { Nord Pool / } \\
\text { Denmark } \\
\text { (reg. up/dn) }\end{array}$ & $\begin{array}{l}\text { No, not } \\
\text { considered }\end{array}$ & Yes, one month \\
\hline $\begin{array}{l}\text { Pinson } \\
\text { et al. } 2007 \text { [17] }\end{array}$ & $\begin{array}{l}\text { Yes, persistence } \\
\text { and fuzzy neural } \\
\text { network }\end{array}$ & $\begin{array}{l}\text { No, fixed } \\
\text { imbalance prices }\end{array}$ & $\begin{array}{l}\text { Min imbalance } \\
\text { cost, analytic } \\
\text { solution }\end{array}$ & $\begin{array}{l}\text { Intervals for } \\
\text { WPF, no price } \\
\text { uncertainty }\end{array}$ & $\begin{array}{l}\text { Europe/ } \\
\text { Netherlands } \\
\text { (regulation up/dn) }\end{array}$ & $\begin{array}{l}\text { No, not } \\
\text { considered }\end{array}$ & Yes, one year \\
\hline $\begin{array}{l}\text { Bourry } \\
\text { et al. } 2008 \text { [20] }\end{array}$ & $\begin{array}{l}\text { Yes, NWP based, } \\
\text { kernel density } \\
\text { estimation (KDE) }\end{array}$ & $\begin{array}{l}\text { Simple deter- } \\
\text { ministic forecast } \\
\text { for imb. prices }\end{array}$ & $\begin{array}{l}\text { Min. imbalance } \\
\text { cost, CVAR }\end{array}$ & WP: KDE & $\begin{array}{l}\text { Denmark } \\
\text { (regulation up/dn, } \\
\text { one-way penalty }\end{array}$ & No & Yes, one year \\
\hline $\begin{array}{l}\text { Usaola and } \\
\text { Moreno } 2009 \\
{[105]}\end{array}$ & $\begin{array}{l}\text { Yes, from the } \\
\text { Sipreolico model }\end{array}$ & $\begin{array}{l}\text { Yes, simple price } \\
\text { prediction }\end{array}$ & $\begin{array}{l}\text { Expected profit, } \\
\text { no risk }\end{array}$ & $\begin{array}{l}\text { Discrete pdf for } \\
\text { WP, pdf for } \\
\text { prices }\end{array}$ & $\begin{array}{l}\text { Spain (regulation } \\
\text { up/dn) }\end{array}$ & $\begin{array}{l}\text { Focus on } \\
\text { intraday, not DA }\end{array}$ & Yes, three months \\
\hline $\begin{array}{l}\text { Morales } \\
\text { et al. } 2010 \text { [106] }\end{array}$ & $\begin{array}{l}\text { No, general } \\
\text { probabilistic } \\
\text { model (AR(2)) }\end{array}$ & $\begin{array}{l}\text { No, general } \\
\text { probabilistic } \\
\text { model (ARIMA) }\end{array}$ & $\begin{array}{l}\text { Profit (hourly) or } \\
\text { CVAR metric } \\
\text { (daily) }\end{array}$ & $\begin{array}{l}\text { Scenario tree } \\
\text { w/reduction, } \\
\text { price correlation }\end{array}$ & $\begin{array}{l}\text { Spain (regulation } \\
\text { up/dn) }\end{array}$ & $\begin{array}{l}\text { Yes, one } \\
\text { adjustment } \\
\text { market }\end{array}$ & $\begin{array}{l}\text { No, one day } \\
\text { analysis }\end{array}$ \\
\hline $\begin{array}{l}\text { Argonne model } \\
\text { in this chapter } \\
{[24][102]}\end{array}$ & $\begin{array}{l}\text { Yes, probabilistic } \\
\text { (quantile regres- } \\
\text { sion or KDE } \\
\text { w/MC sampling) }\end{array}$ & $\begin{array}{l}\text { Yes, normal dist. } \\
\text { based on price } \\
\text { history for DA } \\
\text { and RT prices }\end{array}$ & $\begin{array}{l}\text { Profit, CVAR, or } \\
\text { Utility (hourly) }\end{array}$ & $\begin{array}{l}\text { WP: Discrete pdf, } \\
\text { Prices: Random } \\
\text { sampling w/DA- } \\
\text { RT correlation }\end{array}$ & $\begin{array}{l}\text { United States } \\
\text { (LMP, two } \\
\text { settlement), DA } \\
\text { deviation penalty }\end{array}$ & No & $\begin{array}{l}\text { Yes, up to four } \\
\text { months in current } \\
\text { analysis }\end{array}$ \\
\hline
\end{tabular}


A wind power producer is facing three main uncertainties when bidding into the DA market: the DA LMP, the RT LMP, and the RT delivery of wind power. We assume that a potential deviation penalty depends on the absolute deviation between the RT delivery and the DA schedule. $^{21}$ Then, if we define a set of joint samples, $m=1 \ldots M$, of LMPs and wind power delivery, a simple formulation of the wind power producer's profit for a given hour, $h$, and sample, $m$, is:

$$
\pi_{h}^{m}=p_{D A, h}^{m} \cdot q_{D A, h}+p_{R T, h}^{m} \cdot\left(q_{d, h}^{m}-q_{D A, h}\right)-p^{2} n_{D A} \cdot\left|q_{d, h}^{m}-q_{D A, h}\right|
$$

Note that a wind power producer has an incentive to curtail its generation whenever the RT price drops below 0 , in order to avoid selling its generation at a negative price. ${ }^{22}$ This is the case regardless of the position in the DA market, since the producer can buy back energy in the RT market at a negative price for the energy scheduled DA. Experience shows that negative RT prices may occur quite frequently in markets with LMPs and a high penetration of wind power. ${ }^{23}$ The model allows for three different assumptions of how the wind power is controlled when RT prices $^{24}$ are low and negative:

1) The wind farm generates at maximum possible output regardless of the RT price.

2) The wind farm shuts down generation whenever the RT price is negative, as shown in (7-2).

3) The wind farm maximizes profits during low and negative prices, also considering a potential deviation penalty, as shown in (7-3).

$$
\begin{aligned}
& q_{d, h}^{m}=\left\{\begin{array}{cl}
\bar{q}_{d, h}^{m}, & p_{R T, h}^{m} \geq 0 \\
0, & p_{R T, h}^{m} \leq 0
\end{array}\right. \\
& q_{d, h}^{m}= \begin{cases}\bar{q}_{d, h}^{m} & , \quad p_{R T, h}^{m} \geq p e n_{D A} \\
\min \left(\bar{q}_{d, h}^{m}, q_{D A, h}\right), & \left|p_{R T, h}^{m}\right|<p e n_{D A} \\
0 \quad & p_{R T, h}^{m} \leq- \text { pen }_{D A}\end{cases}
\end{aligned}
$$

\footnotetext{
${ }^{21}$ The model allows for an alternative penalty representation, where the RT price is adjusted according to a penalty factor and there is a deadband where no penalty applies. However, this representation has not been thoroughly tested and is not used in the case study presented in this chapter.

${ }^{22}$ In many cases, wind power producers receive additional benefits, such as production tax credits or renewable energy credits. In these cases, they have an incentive to produce even with negative prices, as long as the net profit remains positive. We do not consider the impact of such incentives here.

${ }^{23}$ For example, hourly price data for the Minnesota hub in MISO for 2009 show that negative LMPs occurred 443 times in the RT market, but only 29 times in the DA market. We ignore the possibility of negative DA prices here. The negative prices in the RT market may to some extent be linked to the wind power benefits discussed in the footnote above.

${ }^{24} \mathrm{New}$ wind power plants can easily be controlled to reduce or shut down their generation, and this flexibility will add to their revenue stream. Older wind turbines may not have this flexibility.
} 
The wind power producer needs to decide on how much to bid into the DA market, $q_{D A, h}$. We assume that the DA offer is bid in at zero price. Hence, the bid will always be accepted, since we assume that the DA price is positive. We can re-write the wind power sample profit in (7-1) to the following:

$$
\pi_{h}^{m}=p_{D A, h}^{m} \cdot q_{d, h}^{m}+\left(p_{R T, h}^{m}-p_{D A, h}^{m}\right) \cdot\left(q_{d, h}^{m}-q_{D A, h}\right)-p e n_{D A} \cdot\left|q_{d, h}^{m}-q_{D A, h}\right|
$$

In this formulation, the first part $\left(p_{D A, h}^{m} \cdot q_{d, h}^{m}\right)$ is equal to the profit if all of the actual wind power generation is settled at the DA price. This corresponds to offering a perfect forecast into the DA market. The latter part of (7-4) is the consequence of deviating from a perfect forecast. Here, the deviation from the forecast is settled at the difference between the RT and DA LMPs, minus the potential deviation penalty. Note that the first part of (7-4) is entirely stochastic. The decision variable, $q_{D A, h}$, occurs in only the latter part of (7-4). If LMPs and wind power delivery are known, we can see from (7-4) that:

1) It is beneficial to deviate from a perfect forecast if the difference between the DA and RT LMPs is larger than the deviation penalty. ${ }^{25}$

2) In the absence of a deviation penalty, the profit maximizing bid is directly dictated by the difference in DA and RT prices. If the DA price is higher (lower) than the RT price, the producer benefits from bidding all (none) of its capacity in the DA market. Hence, the optimal bid is independent of the wind power delivery and, therefore, also independent of the wind power forecast.

The net deviation settlement as a function of the RT deviation is illustrated in Fig. 7-1. The figure shows the deviation settlement for different assumptions about the difference between DA and RT LMPs with and without a deviation penalty. When there is no deviation penalty (left), it is evident that the wind power producer would want to maximize the deviation and bid into the DA market according to the price difference between the two markets. A deviation penalty (right) gives an incentive to minimize the deviation as long as the expected price difference is smaller than the penalty. Note that the deviation settlement is not symmetric when there is an LMP difference.

Of course, the LMPs and the wind power delivery are unknown at the time of bidding into the DA market. A wind power producer therefore has to consider the combined risk from these uncertainties when deciding on a bidding strategy. All of the three stochastic variables are taken into account in the bidding model presented in Section 7.5.

\footnotetext{
${ }^{25}$ Note that this approach is different from most market designs in Europe, with different balancing prices for realtime deviations up and down, so the wind power producer cannot benefit by deviating from the advance schedule.
} 

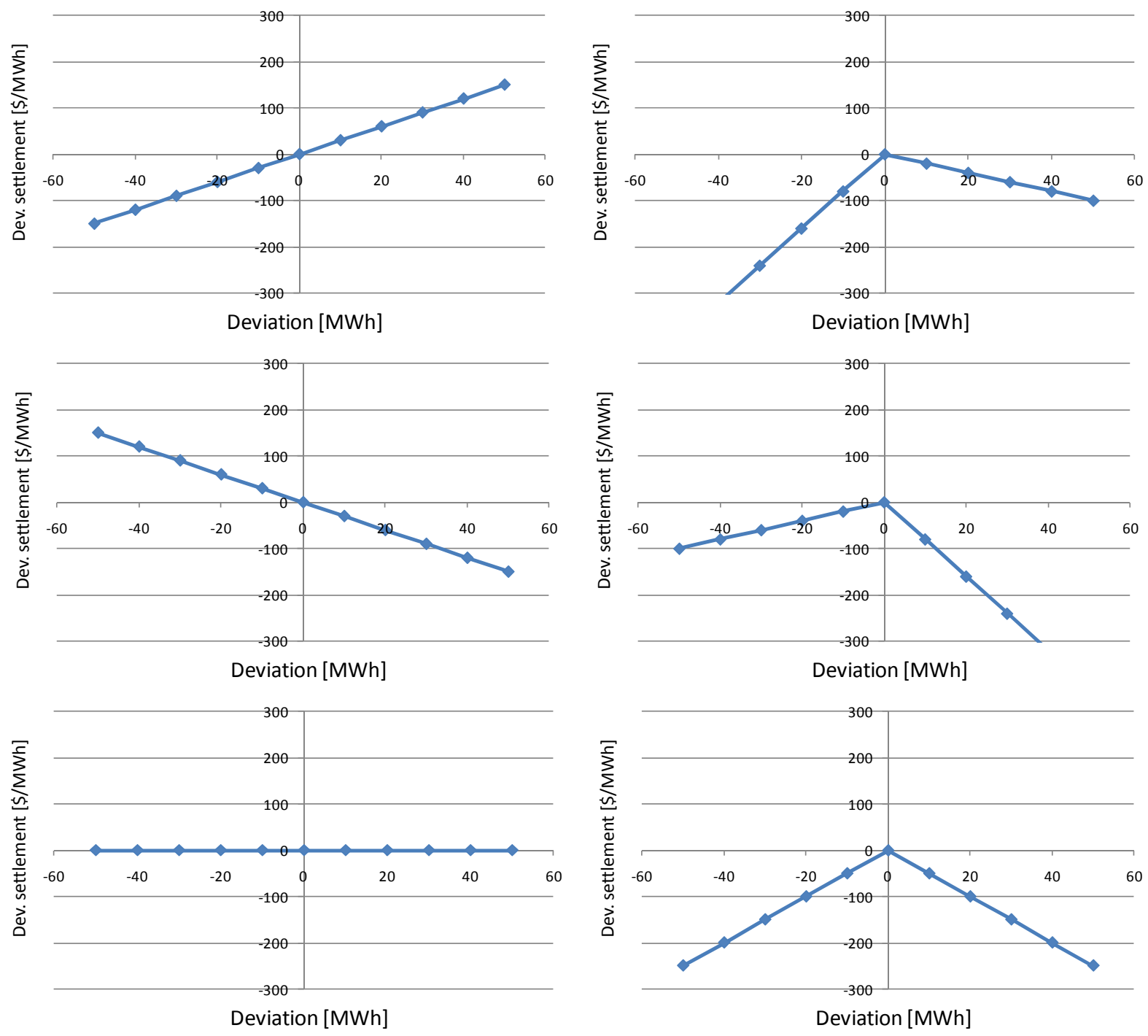

Fig. 7-1 Examples of hourly financial settlements as function of deviation between RT delivery and DA schedule with penalties: $\operatorname{pen}_{D A}=\mathbf{0}$ (left), and $p e n D A=\mathbf{5} \$ / \mathbf{M W h}$ (right), and LMP differences: $p_{R T, h}^{m}-p_{D A, h}^{m}=3,-3,0 \$ / M W h$ (upper, middle, lower).

\subsection{Probabilistic Wind Power Forecasting}

The uncertainty in the wind power forecast is obviously of importance for a wind power producer bidding into the electricity market, since the realized wind generation and the deviation from the DA bid determine the profits as discussed above. There are several different approaches for representing uncertainty in the projected wind power, as explained in [1], including probabilistic representations, risk indices, and scenarios. The wind power trading model presented below can take any discrete probabilistic representation of the wind power forecast. In our work, we use statistical approaches to estimate forecast uncertainty based on historical forecasting errors. We have applied both quantile regression $(\mathrm{QR})$ and $\mathrm{KDE}$ to model forecast uncertainty, as briefly outlined below. 
In the case study included in this chapter, we use the QR approach from [77] as the main approach to produce probabilistic forecasts based on the wind power point forecast errors. The model employs a linear quantile regression with the base functions formulated as cubic B-splines in order to obtain the quantile with a proportion of the forecast errors. Each quantile is modeled as a sum of the nonlinear smooth functions of the forecasted wind power generation. Spline bases are used to approximate each of the smooth functions as a linear combination of base functions. The probabilistic forecast is represented through a set of quantiles for each hour of the day ranging from 0.05 to 0.95 with a 0.05 increment.

The method described in [78] is used to generate a number of wind power scenarios that provide information on the dependency of the prediction errors through the set of look-ahead times. The method is based on the conversion of the set of random variables composing probabilistic forecast series, as obtained with the QR method described above, into a multivariate Gaussian random variable. The temporal interdependence structure is represented by the covariance matrix, which is recursively estimated because of the nonstationary characteristics. Monte Carlo simulation is used for the generation of equiprobable scenarios. Fig. 7-2 and Fig. 7-3 illustrate the resulting forecast quantiles (which are equivalent to forecast intervals) and scenarios of wind power generation, respectively.

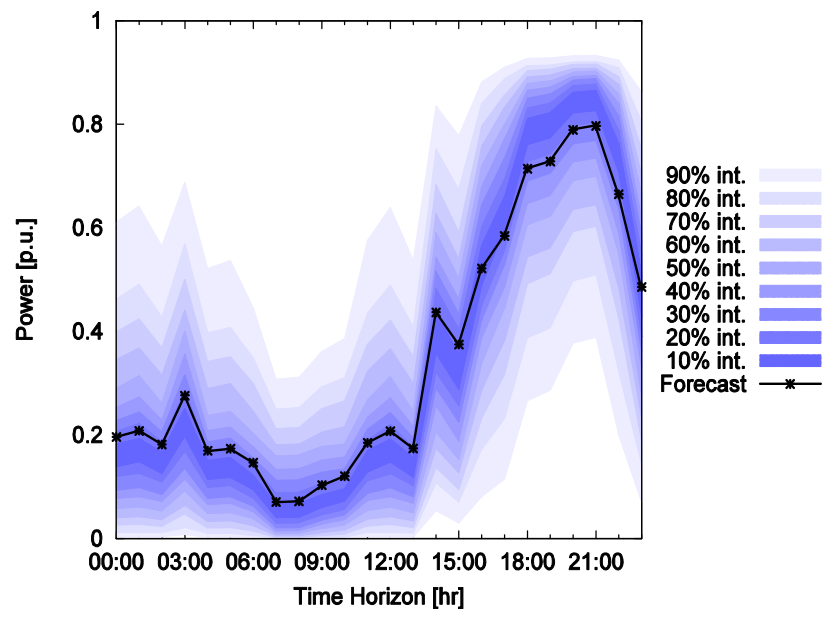

Fig. 7-2 Probabilistic representation of wind power forecast as intervals or quantiles.

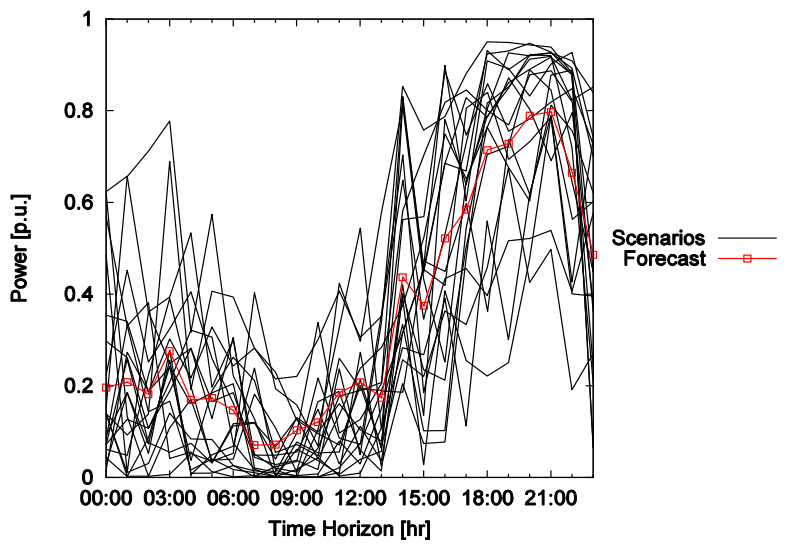

Fig. 7-3 Forecast scenarios of wind power generation. 
In this project, we have developed novel time-adaptive KDE algorithms for probabilistic WPF, based on the Nadaraya-Watson (NW) [93] and quantile copula (QC) [94] [95] estimators. The $\mathrm{KDE}$ algorithms can also be used to estimate the forecast uncertainty as input to the wind power trading model. In this case, we use conditional kernel density estimation to produce a nonparametric probabilistic wind power forecast, from which we derive hourly samples for available wind power generation for each hour of the next day, $\bar{q}_{d, h}^{m}$. In short, conditional density estimation consists of estimating the density of a random variable $Y$, knowing that the explanatory random variable $X$ is equal to $x$. For the wind power problem, it consists of forecasting the wind power probability density function $(p d f)$ at time step $t$ for each look-ahead time step $t+k$ of a given time-horizon when a set of explanatory variables (e.g., numerical weather prediction variables and current wind power) is known. When translated to an equation, we have:

$$
\hat{f}_{P}\left(p_{t+k} \mid X=x_{t+k \mid t}\right)=\frac{f_{P, X}\left(p_{t+k}, x_{t+k \mid t}\right)}{f_{X}\left(x_{t+k \mid t}\right)}
$$

where $p_{t+k}$ is the wind power forecasted for look-ahead time $t+k, x_{t+k \mid t}$ is the explanatory variable forecasted for look-ahead time step $t+k$ and available at time step $t, f_{P, X}\left(p_{t+k}, y_{t+k \mid t}\right)$ is the multivariate density function, and $f_{X}(x)$ is the marginal density of $X$. The output is the forecasted $p d f$ for wind power generation, which is then converted into a probability mass function ( $p m f$ ) with a set of $M$ equally probable points for each hour. Scenario generation is not required, as each hour is treated independently in the wind power trading problem, as further discussed below. More details about the KDE forecasting methods can be found in [93]-[95]. In the case study, we do a brief comparison of applying QR and the two KDE methods to the wind power trading problem.

\subsection{A Model for Wind Power Trading under Uncertainty}

Our wind power trading model optimizes the quantity to bid into the DA market for a wind power producer. We use a discrete representation of uncertainty in the hourly wind power forecast, as outlined in the previous section. DA and RT LMPs are also represented as discrete probability distributions, as explained below. We use the wind power and LMP information to calculate discrete probability distributions for the wind power producer's profit as a function of the DA bid. This allows us to derive optimal DA bids under different assumptions for the decision maker's risk preferences.

The initial ideas for the wind power trading model were first presented in [24]. Since then, we have extended the model and analysis in multiple directions. We use different statistical approaches for probabilistic WPF, and we have developed a more complete description of the decision problem. We have conducted a more detailed case study based on hypothetical wind farm data, as documented in the next section. We have also applied the model to a case study of a real-world wind farm [102]. 


\subsubsection{Representation of Uncertainty in DA and RT LMPs}

We use a straightforward model to project DA and RT LMPs for the next day. The price forecasts are based on historical LMPs. ${ }^{26} \mathrm{~A}$ window of four weeks from the most recent history is used to estimate the mean, variance, and correlation for each individual hour of the next day. Weekdays and weekend days are treated independently. Hourly samples of DA and RT LMPs are then drawn from (7-6) and (7-7) with the estimated parameters. Note that the same two sets of random samples $\left(\varepsilon_{D A}^{m}, \varepsilon_{R T}^{m}\right)$ are used in all hours:

$$
\begin{aligned}
& p_{D A, h}^{m}=\varepsilon_{D A}^{m} \cdot \sigma_{D A, h}+\mu_{D A, h} \\
& p_{R T, h}^{m}=\left(\rho_{h} \cdot \varepsilon_{D A}^{m}+\sqrt{1-\rho_{h}{ }^{2}}\right) \cdot \varepsilon_{R T}^{m} \cdot \sigma_{R T, h}+\mu_{R T, h}
\end{aligned}
$$

This discrete representation of the uncertainty in LMPs assumes that prices follow a normal distribution. However, any probability distributions could be used, as long as the distributions are converted to discrete samples. A set of $M$ joint random samples for DA and RT LMPs are generated and paired with the wind power $p m f$ to form a total set of $M$ samples for all three random variables. Note that the formulation in (7-6) and (7-7) includes the correlation between DA and RT LMPs. In areas with large shares of wind power, there could also be a significant negative correlation between LMPs and wind power. The wind-LMP correlation could also be captured. For instance, a more elaborate price forecasting system that uses the wind power forecast as input can be found in [111]. A more advanced price forecasting system could easily be incorporated into the model, as long as the price forecasts have a discrete representation.

\subsubsection{Mathematical Formulation of the DA Bidding Problem}

The objective for the wind power producer is to find the optimal DA bid, $q_{D A, h}$, depending on the decision criterion adopted. We consider three different objective functions for the problem, as shown in (7-8)-(7-10): (1) expected profit, (2) CVAR [112], and (3) expected utility [113]. The decision maker's risk preferences are reflected in the choice of objective function and corresponding parameters. The expected profit criterion in (7-8) is risk neutral. The CVAR criterion in (7-9) is risk averse, since the lower part of the profit distribution (i.e., CVAR) carries additional weight in the objective function. For the utility criterion, we assume a standard exponential utility function [113], as shown in (7-10). The utility function can range from risk prone to risk averse, depending on the $\beta$ parameter.

$$
\begin{aligned}
& E_{h}^{*}=\operatorname{Max}_{q_{D A, h}} \sum_{m=1}^{M} \operatorname{prob}_{m} \cdot \pi_{h}^{m}\left(q_{D A, h}\right) \\
& C_{h}^{*}=\operatorname{Max}_{q_{D A, h}}\left\{\sum_{m=1}^{M} \operatorname{prob}_{m} \cdot \pi_{h}^{m}\left(q_{D A, h}\right)+w \cdot C V A R_{h}\left(q_{D A, h}, t h\right)\right\}
\end{aligned}
$$

\footnotetext{
${ }^{26}$ We also experimented with more advanced ARIMA models for LMP projections, but since they did not give significant improvements in forecast accuracy, we chose to use the simpler approach for price projections.
} 
where

$$
\begin{array}{r}
\operatorname{CVAR}_{h}\left(q_{D A, h}, t h\right)=\mathrm{E}\left(\pi_{h} \mid \pi_{h}<\pi_{h}^{q u a n t, t h}\right) \\
U_{h}^{*}=\operatorname{Max}_{q_{D A, h}} \sum_{m=1}^{M} \operatorname{prob}_{m} \cdot U_{h}^{m}\left(\pi_{h}^{m}\left(q_{D A, h}\right)\right)
\end{array}
$$

where

$$
U_{h}^{m}=\frac{1}{1-e^{\beta}} \cdot\left[1-e^{\frac{\beta\left(\pi_{h}^{m}-\pi^{m i n}\right)}{\pi^{m a x}-\pi^{m i n}}}\right]
$$

In all three objective functions, the sample profit, $\pi_{h}^{m}$, is given by (7-1). Constraints (7-2) or (7-3) apply depending on the assumption of wind power control during negative prices, as discussed above. In addition, we impose that the DA bid must be below the normalized capacity of the wind farm (i.e., $0 \leq q_{D A, h} \leq 1$ ). In general, the resulting optimization problem is a nonlinear programming problem. We use the generalized reduced gradient algorithm built into Excel's solver to find the solution. ${ }^{27}$ The model currently has a simple user interface in Excel (Fig. 7-4), and is a stand-alone Excel application. Multi-hour simulations can be conducted to analyze the performance of the different bidding strategies over time, as illustrated in the case study in the next section.

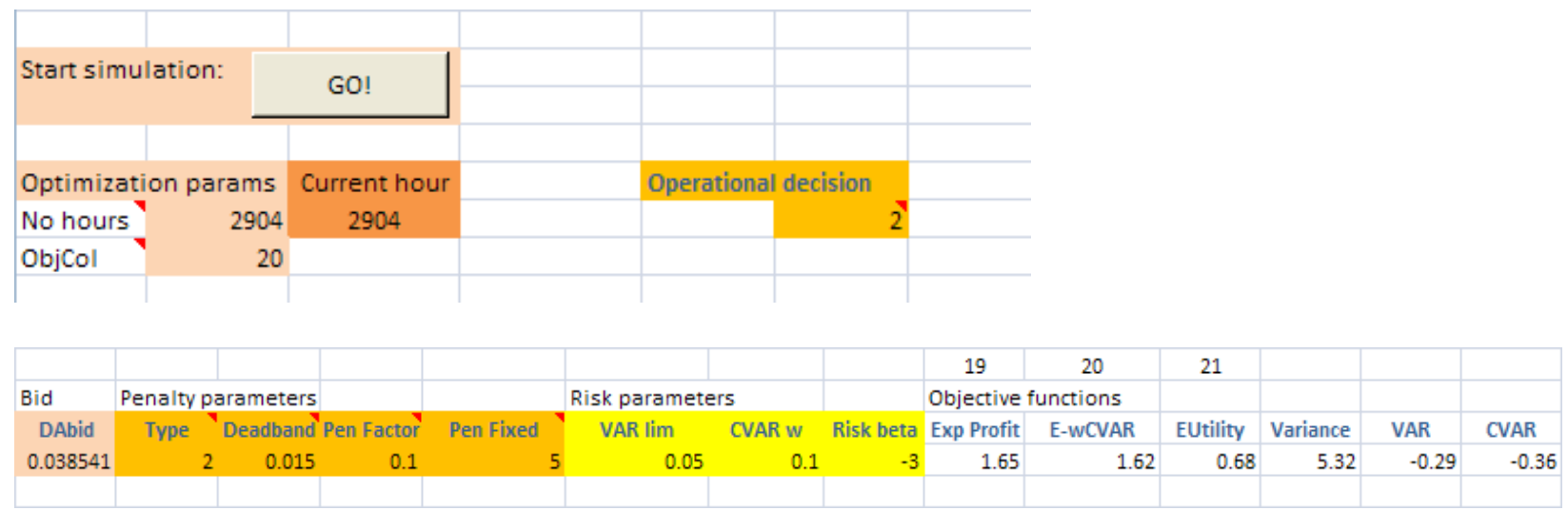

Fig. 7-4 Excerpts from Excel interface.

\footnotetext{
${ }^{27}$ A common problem with nonlinear optimization models is the possibility of convergence to a local optimum. This problem applies to the reduced gradient algorithm as well, and it cannot guarantee that the solution is a global optimum. However, the optimization problem only has one decision variable, which is constrained between 0 and 1 . Possible local optima can therefore easily be identified and ruled out (experience shows that the problem occurs most frequently with a risk-prone utility function). An initial starting point for the search is also provided to guide the algorithm in the right direction.
} 


\subsection{Case Study: Hypothetical Wind Farm in Illinois}

In this section, we apply the model outlined above in a case study of a hypothetical wind farm in the state of Illinois. We analyze how DA bids depend on risk preferences and deviation penalty schemes, assuming that wind power is sold as merchant generation.

\subsubsection{Assumptions}

We analyze the bidding of a hypothetical wind farm in the state of Illinois, using wind power data from one location (site 4848) in the Eastern Wind Integration and Transmission Study (EWITS) [83]. The EWITS database provides both point forecast and realized wind power generation. The underlying data methodology is explained in [84]. Data from January to August 2006 are used to train the quantile regression method [77] in order to generate probabilistic forecasts. A total of 1,000 scenarios of wind power generation are generated for each hour of the day for October, November, and December using the Monte-Carlo sampling scheme from [78]. The deterministic point forecast and 10 forecast scenarios are shown for one selected day in Fig. 7-5. The figure illustrates that forecast scenarios span a relatively wide range around the point forecast.

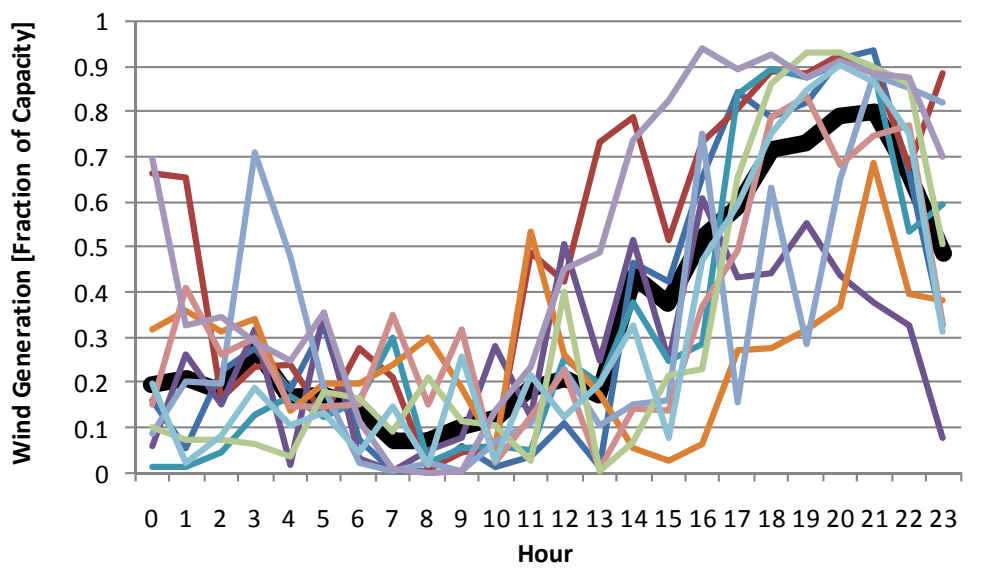

Fig. 7-5 Wind power forecast: Deterministic point forecast (solid thick line) and 10 forecast scenarios for selected day (Oct. 9, 2006).

We use market price data from the Illinois trading hub in the MISO to estimate the price parameters for each individual hour with the methodology outlined in Section 7.5.1. Fig. 7-6 shows the resulting price parameters for October 9. Monte-Carlo simulation generates 1,000 scenarios for DA and RT prices for each hour. The price scenarios are combined with the wind power scenarios described above. The resulting set of scenarios is used to evaluate DA bidding, market profits, and risk measures under different decision criteria. Illustrations of price scenarios are shown in Fig. 7-7, with RT prices clearly having a wider range of uncertainty than DA prices. Note that the uniformity between the hours occurs because the same random sample is used for all hours of each day. Capturing the inter-temporal correlation is not important in this problem because each hour is optimized individually. 


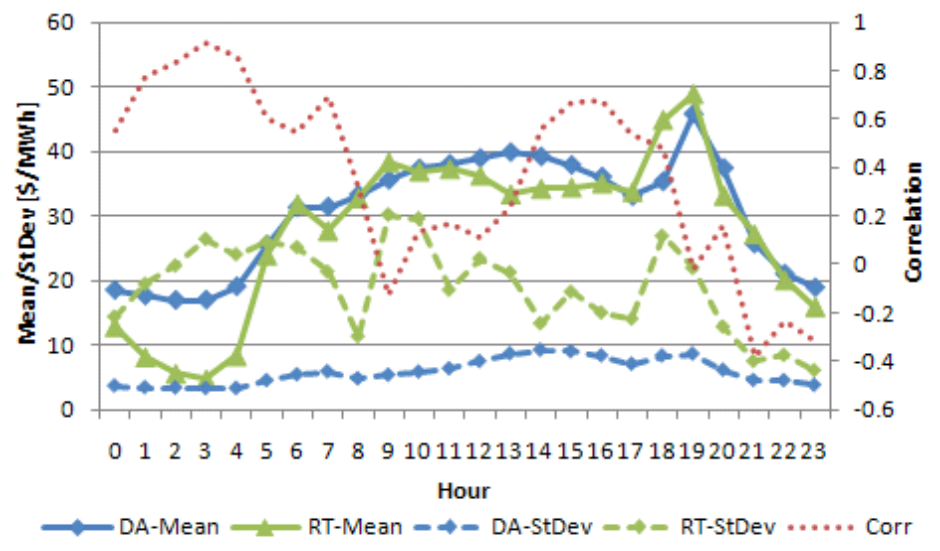

Fig. 7-6 Estimated DA and RT price parameters for selected day (Oct. 9, 2006).
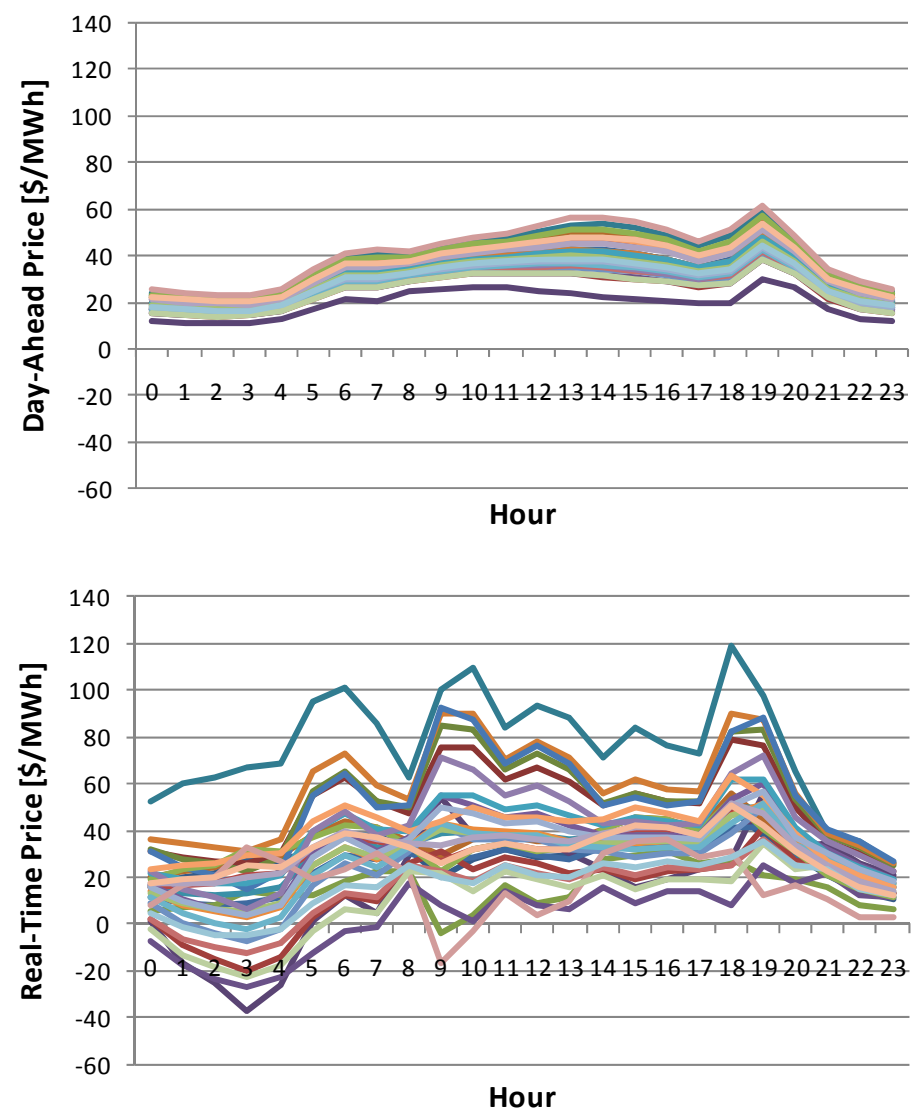

Fig. 7-7 Thirty scenarios for DA (upper) and RT (lower) prices for selected day (Oct. 9, 2006).

\subsubsection{Results}

We first present results for one individual hour, looking at optimal strategies and risk-return trade-offs for different decision criteria. We then calculate the optimal bidding decisions for all the hours of the day under different assumptions for decision criteria and deviation penalty. Finally, we present results where the effectiveness of the different strategies are evaluated over a one-month period against actual historical price data. The default assumption in this case study is that the wind power producer curtails the output during negative prices, according to (7-2). For 
the CVAR decision criterion in (7-9), we assume a CVAR threshold, $t h$, of 5\% and a weight, $w$, of 0.1 . For the utility decision criterion in (7-10), we assume that the risk parameter, $\beta$, is either -3 (risk averse) or +3 (risk prone). The min-max profit range in the utility function is set to $[-100,120]$. These values are chosen for illustrative purposes. In reality, the choice of decision criteria and corresponding parameters should reflect the actual risk preferences of the wind power producer.

\subsubsection{Analysis of Bidding in Selected Hour}

We now investigate the bidding problem for one selected hour (i.e., hour 5 on October 9 (Fig. 7-5 through Fig. 7-7) in more detail. Table 7-2 shows that the expected DA price is slightly higher than the expected RT price for this hour. However, the RT price is much more volatile, resulting in a much larger spread in RT price scenarios as compared to DA (Fig. 7-7). There is also a significant correlation between DA and RT prices. The wind power forecast is relatively low, with only $17.4 \%$ of installed capacity expected to be available. However, the 1,000 wind power scenarios span a wide range from 0 to $72.8 \%$ of installed capacity.

Table 7-2 Price parameters and wind power point forecast (pf) for hour 5, Oct. 9, 2006.

\begin{tabular}{|c|c|c|c|c|c|c|c|}
\hline$\mu_{D A, 5}$ & $\mu_{R T, 5}$ & $\sigma_{D A, 5}$ & $\sigma_{R T, 5}$ & $\rho_{5}$ & $p f_{5}$ & $\bar{q}_{d, 5}^{\max }$ & $\bar{q}_{d, 5}^{\min }$ \\
\hline 25.49 & 23.96 & 4.48 & 25.91 & 0.60 & 0.174 & 0.728 & 0.002 \\
\hline
\end{tabular}

The objective functions for different decision criteria are shown in Fig. 7-8 and Fig. 7-9. For the expected value criterion (E), it is evident that that the optimal DA bid is to bid all capacity in the DA market. This result is not surprising given that $\mu_{D A, 5}>\mu_{R T, 5}$. In this case, it will always be optimal to bid maximum capacity in the DA market under the profit criterion, regardless of the wind power forecast, as long as there is no deviation penalty. The CVAR criterion (C) yields an optimal bid just slightly above zero. This is because the CVAR decreases as a function of bid quantity (Fig. 7-10). The optimal bid under the risk-averse utility criterion is 0.39 , whereas it is the same as under expected profit with a risk-prone utility function. The results clearly illustrate that the optimal bidding decisions depend on the risk preferences of the decision maker.

The level of risk for different bidding quantities is further explored in Fig. 7-10. The figure shows that the variance in profit is lowest around the point forecast level of wind generation. The profit variance increases quickly as a function of higher amounts of bidding in the DA market. Although the variance also increases for bids below the point forecast, the increase is relatively modest. In this case, the producer is likely to sell some of its generation in the RT market. Still, the producer can hedge against selling at negative prices in RT by not producing and thereby controlling some of the risk. In contrast, the producer cannot hedge against buying at high RT prices, which is more likely to occur the more power is bid into the DA market. This explains the high variance for high bidding quantities. It also explains why the one-sided CVAR measure declines as a function of $q_{D A, 5}$. Fig. 7-10 also indicates the impact on variance and CVAR from curtailing wind at negative prices. Curtailment clearly reduces CVAR considerably for low bidding quantities, because selling at negative prices in the RT market is avoided, reducing the downside risk. Note that curtailing the wind power when prices are negative also tends to extend the upside of the profit distribution, since the producer benefits from buying at negative prices instead of producing, regardless of the bid quantity. 


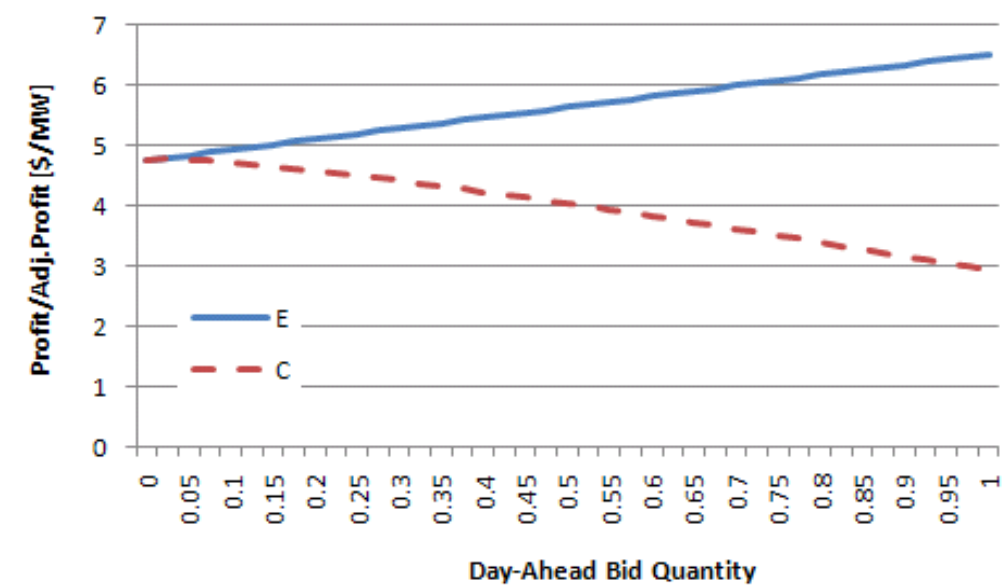

Fig. 7-8 Expected profit (E) and CVAR criteria (C) as function of DA bid quantity, no deviation penalty (hour 5 , Oct. 9,2006 ).

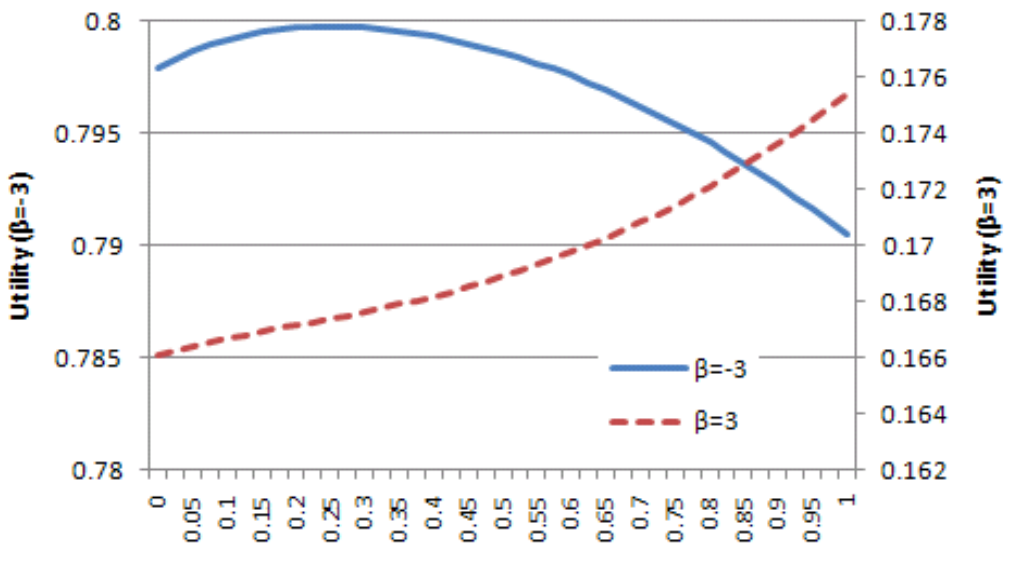

Day-Ahead Bid Quantity

Fig. 7-9 Expected utility for the risk-averse $(\beta=-3)$ and risk-prone $(\beta=3)$ decision maker, no deviation penalty.

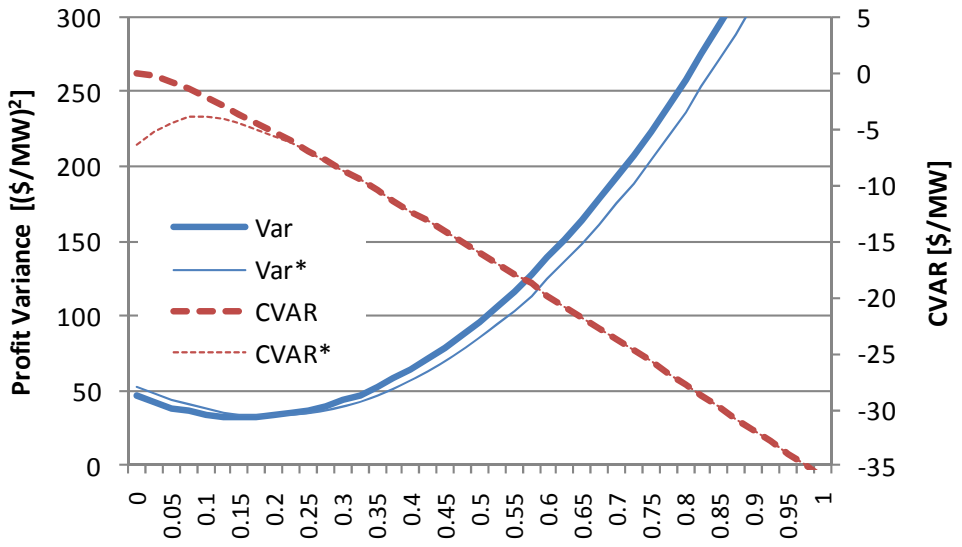

Day-Ahead Bid Quantity

Fig. 7-10 Variance and conditional value at risk, no deviation penalty (hour 5, Oct. 9, 2006). *Without wind power curtailment during negative prices. 
The trade-off between risk and return is further illustrated in Fig. 7-11, which shows the expected profit vs. CVAR for the range of possible DA bids. In this hour, it is clear that all of the bids lie on an efficient frontier. For a decision maker considering these two criteria, the optimal strategy therefore depends on how much weight to assign to each of them. Profit maximizing (i.e., risk neutral) and risk-prone decision makers opt for maximum profit. The optimal strategy according to the conditional value at risk objective is to maximize CVAR, whereas decisions based on point forecast and the risk-averse utility function lie somewhere in between.

Fig. 7-12 shows the same results with a $\$ 5 / \mathrm{MWh}$ deviation penalty. Note that the expected profits are now clearly reduced due to the penalty. Furthermore, only a limited portion of the potential bids is now on the efficient frontier. For instance, the optimal bid according to the riskprone utility function is not on the efficient frontier. This is not surprising, as the utility criterion does not consider CVAR in the objective function. However, the optimal bids under all of the other criteria and the point forecast are on the efficient frontier.

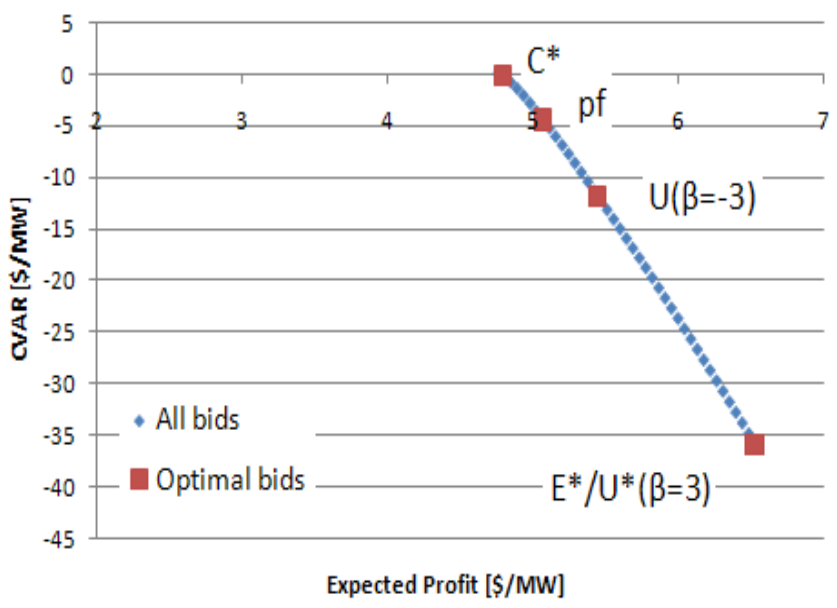

Fig. 7-11 Illustration of the trade-off between expected profit and conditional value at risk, no deviation penalty (hour 5 , Oct. 9, 2006).

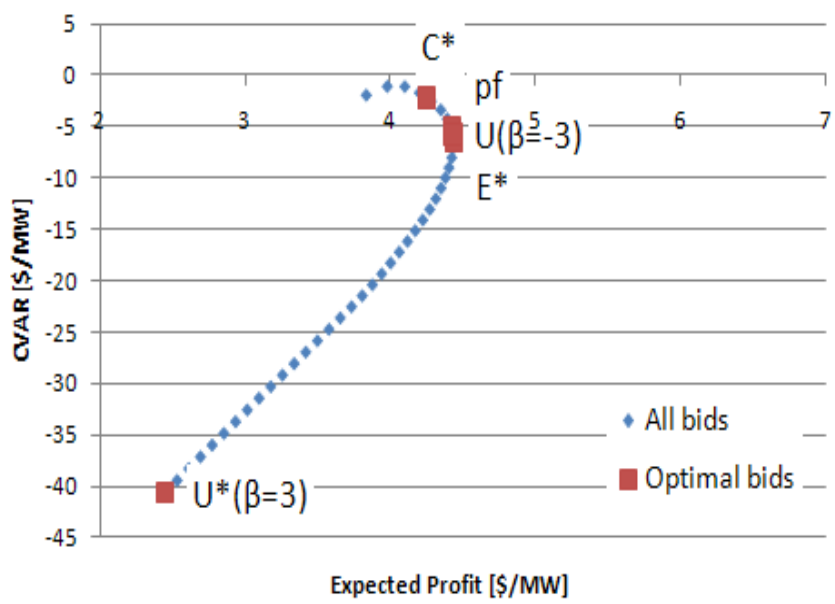

Fig. 7-12 Illustration of the trade-off between expected profit and conditional value at risk, $\$ 5 / \mathrm{MWh}$ deviation penalty (hour 5, Oct. 9, 2006). 
The optimal bidding results for the individual hour are summarized in Table 7-3. Table 7-4 summarizes the results with a $\$ 5 / \mathrm{MWh}$ deviation penalty. The penalty drives the optimal bids closer to the point forecast. At the same time, the expected profit (E) and the CVAR (C) criteria are reduced due to the penalty.

Table 7-3 Summary of results at optimum for different decision criteria (hour 5, Oct. 9, 2006), no deviation penalty.

\begin{tabular}{|l|c|c|c|c|c|c|c|}
\hline Criteria & $q_{D A}{ }^{*}$ & $\pi$ & $C$ & $U^{a}$ & $V A R$ & $C V A R$ & $\sigma_{\pi}$ \\
\hline $\mathrm{E}$ & 1.00 & 6.52 & 2.93 & 0.796 & -26.8 & -36.0 & 419.3 \\
\hline $\mathrm{C}$ & 0.02 & 4.79 & 4.78 & 0.799 & 0.13 & -0.14 & 43.3 \\
\hline $\mathrm{U}(\beta=-3)$ & 0.39 & 5.45 & 4.25 & 0.800 & -7.74 & -11.9 & 61.7 \\
\hline $\mathrm{U}(\beta=3)$ & 1.00 & 6.52 & 2.93 & 0.796 & -26.8 & -36.0 & 419.3 \\
\hline${ }^{a} \beta=-3$ &
\end{tabular}

Table 7-4 Summary of results at optimum for different decision criteria (hour 5, Oct. 9, 2006), $\$ 5 / M W h$ deviation penalty.

\begin{tabular}{|l|c|c|c|c|c|c|c|}
\hline Criteria & $q_{D A}{ }^{*}$ & $\pi$ & $C$ & $U^{a}$ & VAR & CVAR & $\sigma_{\pi}$ \\
\hline $\mathrm{E}$ & 0.21 & 4.44 & 3.79 & 0.798 & -4.28 & -6.47 & 32.4 \\
\hline $\mathrm{C}$ & 0.09 & 4.25 & 4.03 & 0.798 & -1.07 & -2.17 & 30.2 \\
\hline $\mathrm{U}(\beta=-3)$ & 0.19 & 4.43 & 3.85 & 0.798 & 31.0 & -3.64 & -5.75 \\
\hline $\mathrm{U}(\beta=3)$ & 1.00 & 2.45 & -1.61 & 0.781 & -31.5 & -40.6 & 427.5 \\
\hline $\mathrm{a}^{\mathrm{a}} \beta=-3$
\end{tabular}

\subsubsection{Optimal Bidding Strategies for One Day}

We now look at the optimal bidding strategy for the entire day (i.e., 24 hours). Fig. 7-13 shows that under the expected profit criterion, the optimal decision is to bid full capacity in the DA market whenever the expected DA price is above the expected RT price. No capacity is bid into the DA market when the expected RT price is higher than the expected DA price under this criterion. The risk-prone utility criterion gives almost exactly the same results (not included in Fig. 7-13). With the CVAR and risk-averse utility criteria, the optimal bid is in between the maximum and minimum capacity in hours with a small difference in expected DA and RT prices. The bid quantity tends to be higher with the utility criterion than it is with the CVAR criterion. Overall, the decision strategies are mainly driven by the price expectations, as long as there is no deviation penalty.

We repeat the analysis with a deviation penalty of US\$5/MWh and US\$10/MWh (Fig. 7-14 and Fig. 7-15). The results show that a deviation penalty would bring the bids closer to the projected generation (i.e., the point forecast) under the expected profit, CVAR, and risk-averse utility criteria. We can see a similar effect for the risk prone utility criterion (Fig. 7-16); however, the impact of the penalty is less prominent because of the willingness to take more risk.

Finally, the trade-offs between expected profit and CVAR for the full day are illustrated in Fig. 7-17 and Fig. 7-18 for the different bidding strategies. The results show that the expected profit criterion (E) yields the highest expected profit. The risk-averse CVAR and utility criteria lead to lower profit but higher CVAR. Finally, it is interesting to note that the point forecast, $p f$, is not on the efficient frontier for the full day, as the CVAR criteria score higher in both expected profit 
and CVAR. This result is the case both with and without a deviation penalty. Note that profit and CVAR are metrics that can be scaled up and calculated over any time resolution. In contrast, the utility function requires a definition of a minimum-maximum range and therefore cannot be scaled up in the same manner.

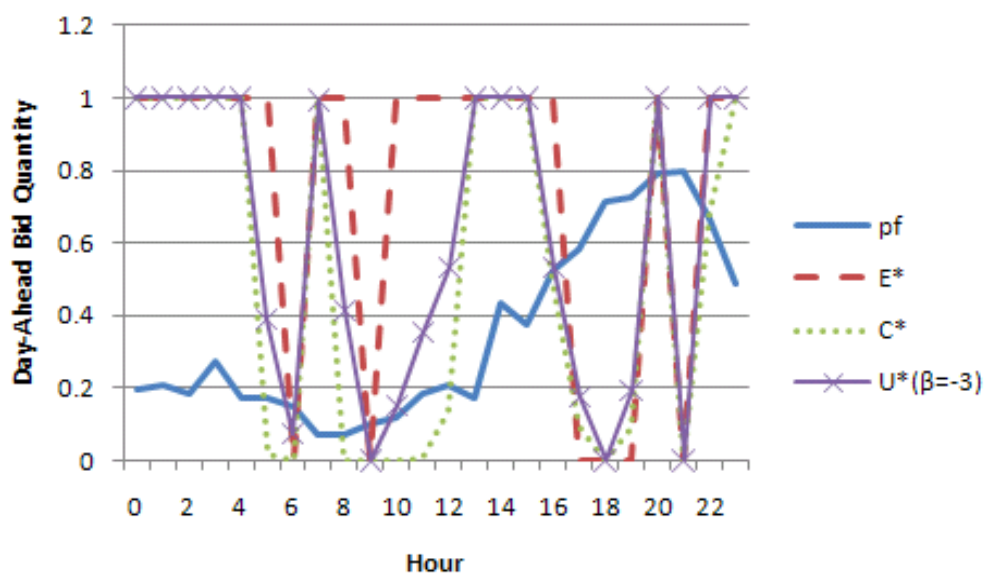

Fig. 7-13 Optimal DA bidding under different decision criteria for 24 hours (Oct. 9, 2006). No deviation penalty. pf is the point forecast.

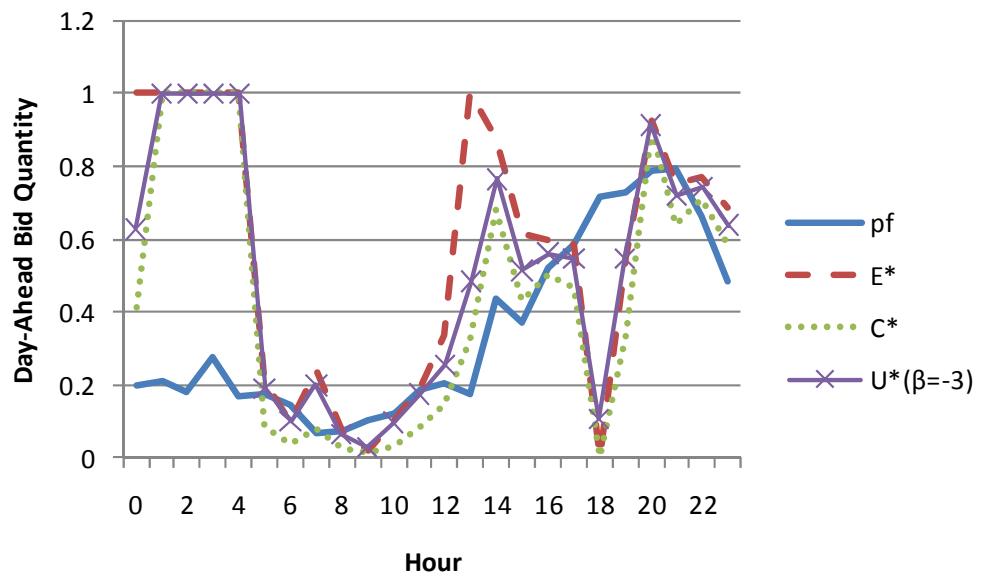

Fig. 7-14 Optimal DA bidding under different decision criteria for 24 hours (Oct. 9, 2006). US $\$ 5 / M W h$ penalty. pf is the point forecast. 


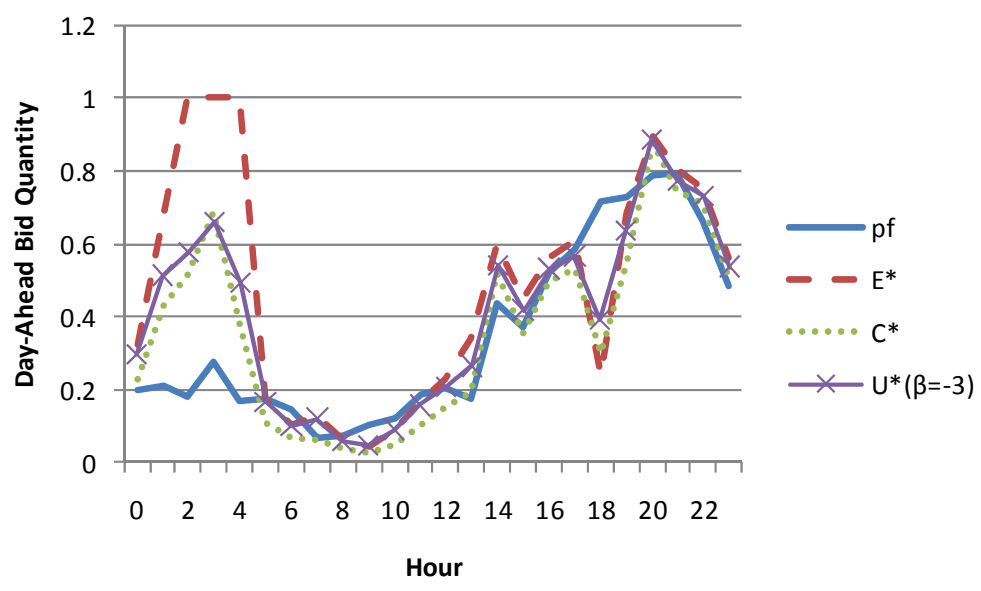

Fig. 7-15 Optimal DA bidding under different decision criteria for 24 hours (Oct. 9, 2006). US $\$ 10 / M W h$ penalty. pf is the point forecast.

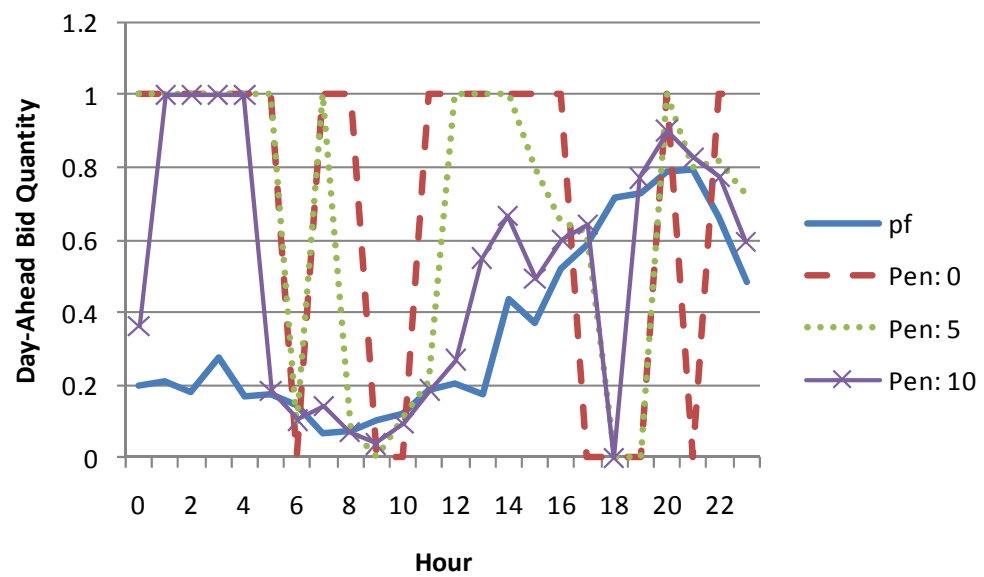

Fig. 7-16 Optimal DA bidding with risk-prone decision criteria $(\beta=3)$ for different deviation penalties $(0,5,10 \$ / M W h)$. (Oct. 9, 2006). pf is the point forecast.

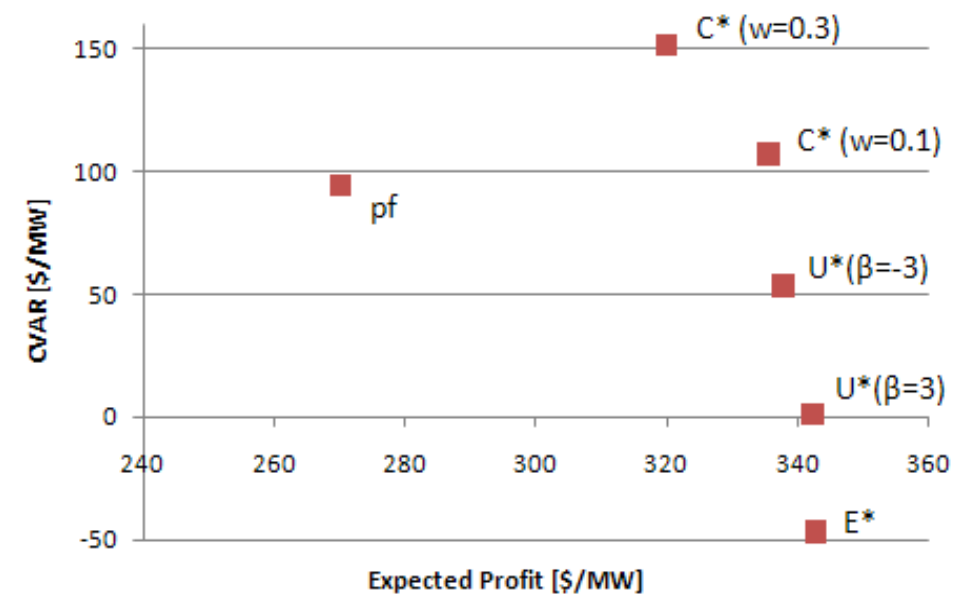

Fig. 7-17 Illustration of the trade-off between expected daily profit and conditional value at risk, no deviation penalty (Oct. 9, 2006). 


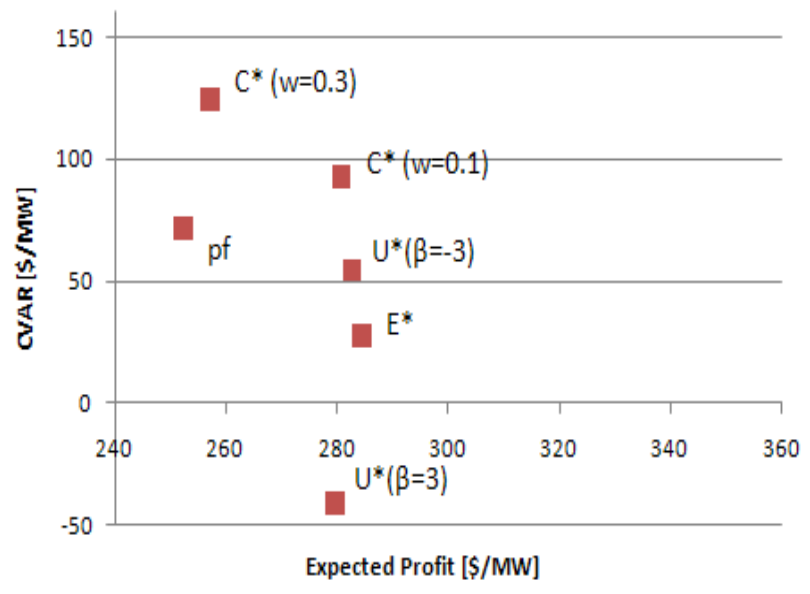

Fig. 7-18 Illustration of the trade-off between expected daily profit and conditional value at risk, $\$ 5 /$ MWh deviation penalty (Oct. 9, 2006).

\subsubsection{Monthly Simulation with Realized Price and Wind Power Outcomes}

In order to assess decisions based on stochastic optimization, it is necessary to analyze a strategy's performance over a range of outcomes. One could do this by sampling from the probability distributions used in the optimization problem. However, a better test for performance in the real world is to use historical realizations of the stochastic variables to calculate realized profit over an extended time period. We have therefore developed a simple simulator in which decision criteria can be tested based on real-world data. The optimal DA bid for each hour is derived based on probabilistic forecasts for wind power and prices, as outlined in Section 7.5. In turn, the realized profit for each hour is calculated with the historical wind power and price data.

In this case study, we have used wind power data (forecast and realized generation) for October 2006 from site 4848 in the EWITS study. We combined the wind power data with DA and RT price data for the Illinois hub at the MISO for October $2009 .^{28}$ Summary statistics for wind power and price data are provided in Table 7-3. Note that RT prices have a much higher volatility and spread as compared to the DA prices. Table 7-5 summarizes the results from testing the wind power trading model with different decision criteria over the one-month period.

Table 7-5 Summary statistics for wind power and DA/RT prices, October.

\begin{tabular}{|l|c|c|c|}
\hline & $\begin{array}{c}\text { Wind Power } \\
{[\% \text { of capacity }]}\end{array}$ & $\begin{array}{c}\text { DA Price } \\
{[\$ / \mathrm{MWh}]}\end{array}$ & $\begin{array}{c}\text { RT Price } \\
{[\$ / \mathrm{MWh}]}\end{array}$ \\
\hline Average & 0.39 & 25.12 & 24.65 \\
\hline Standard Deviation & 0.31 & 8.99 & 15.39 \\
\hline Minimum & 0.00 & 10.40 & -39.10 \\
\hline Maximum & 0.96 & 53.89 & 223.34 \\
\hline
\end{tabular}

\footnotetext{
${ }^{28}$ Note that the wind and price data are from two different years in this analysis. We do not consider the correlation between wind power and price in the probabilistic forecasts. At the same time, the wind power data is for a hypothetical site, so there is no relationship between the price and wind power data. Hence, it is not important to have synchronized time series of prices and wind power in this case study. In current work, we are testing the model on data from a real wind farm, and in this case we do use synchronized wind and price data [102].
} 
We first analyze the results with no deviation penalty. The trade-off between total profit over the month and the CVAR calculated based on the distribution of hourly realized results is shown in Fig. 7-19. The expected profit $\left(E^{*}\right)$ and risk-seeking utility $\left(U^{*}(\beta=0.3)\right)$ strategies give the highest profit but also the lowest CVAR. The CVAR strategy $\left(C^{*}\right)$, in particular with the weight of 0.3 , gives a much higher CVAR, as expected. Note that bidding according to the point forecast (pf) gives much lower profit but has a low risk level. This outcome is also the case for the strategy of constantly bidding zero capacity (zero) into the DA market, thereby selling all of the energy into the RT market.

The trade-off between the wind farm profit on the one hand and the deviation from DA schedule on the other hand is shown in Fig. 7-20. Under most circumstances, the system operator will benefit from keeping the deviations from the DA schedule as small as possible. ${ }^{29}$ Among all of the strategies considered, point forecast bidding gives the lowest absolute deviation from the DA schedule. However, this strategy also yields a low total profit. Hence, in this case there are clearly conflicting objectives between the wind farm owners and the system operator. This result is in line with our analysis in Chapter 2, where we discuss the value of WPF for different market participants and how training criteria for wind power point forecasts can be tuned according to the objectives of the forecast users.

The results for all of the strategies under the assumption of no deviation penalty are summarized in Table 7-8. One interesting observation is that the strategies that consider the price forecasts all bid more into the DA market than the wind power point forecast, as shown by the average DA bid. This is because the bidding strategy is driven by price expectations. The average DA price is higher than the average RT price in the simulation period (Table 7-5), and this is reflected in the price projections. Hence, in the absence of a deviation penalty, the wind power producers bid above the point forecast to take advantage of expected higher DA prices. The price-based strategies result in a higher profit, but also increase the wind power producer's risk exposure measured in terms of the hourly variance and CVAR (Table 7-8).

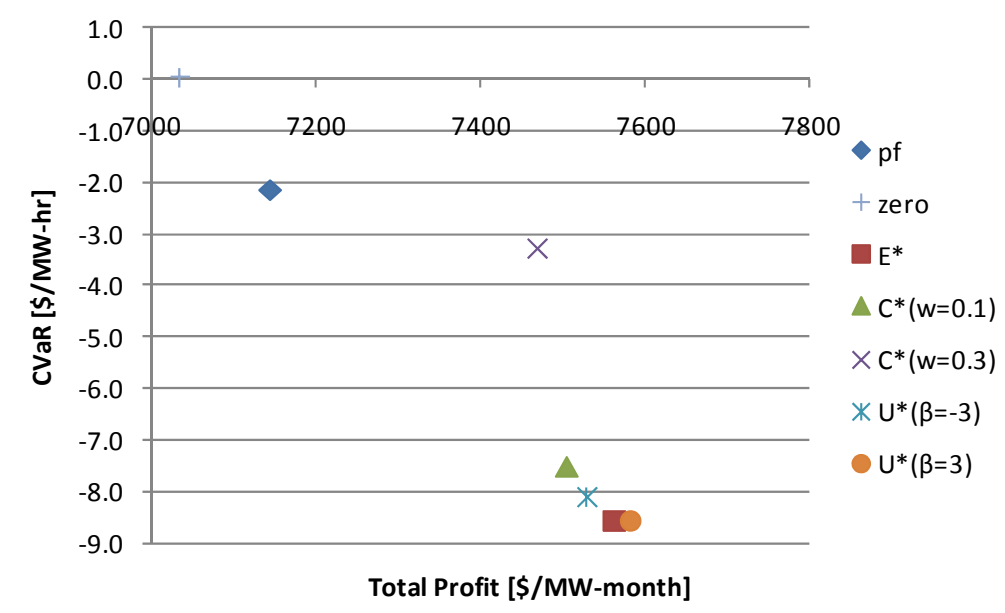

Fig. 7-19 Total October profit vs. hourly conditional value at risk, no deviation penalty.

\footnotetext{
${ }^{29}$ This is not always the case, however, as the system operator could actually benefit if the wind farm deviation goes against the system deviation at the respective location.
} 


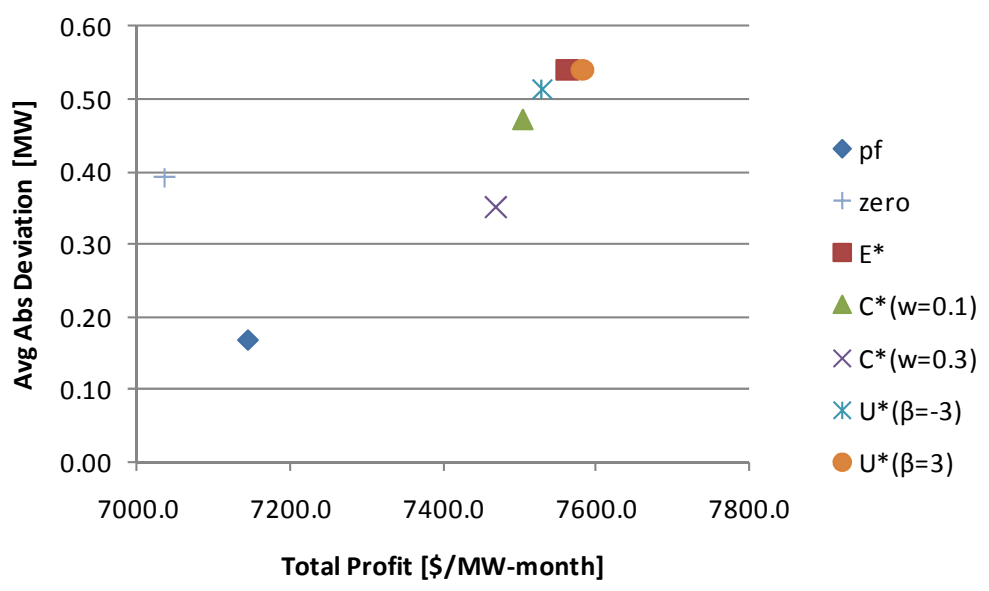

Fig. 7-20 Total October profit vs. average absolute deviation from schedule, no deviation penalty.

Table 7-6 Summary of results for October with different decision criteria, no deviation penalty.

\begin{tabular}{|c|c|c|c|c|c|}
\hline Criteria & $\begin{array}{c}\text { Total Profit } \\
{[\$]}\end{array}$ & $\begin{array}{l}\text { CVAR } \\
{[\$ / \mathrm{hr}]}\end{array}$ & $\begin{array}{c}\text { Variance } \\
{\left[\$^{2} / \mathrm{hr}\right]}\end{array}$ & Avg. Bid & $\begin{array}{c}\text { Avg. } \\
\text { Deviation }\end{array}$ \\
\hline $\mathrm{pf}$ & $7,145.2$ & -2.17 & 78.7 & 0.38 & 0.17 \\
\hline zero & $7,035.3$ & 0.03 & 78.0 & 0.00 & 0.39 \\
\hline $\mathrm{E}^{*}$ & $7,562.3$ & -8.57 & 105.9 & 0.71 & 0.54 \\
\hline$C^{*}(w=0.1)$ & $7,504.7$ & -7.53 & 104.9 & 0.60 & 0.47 \\
\hline$C^{*}(w=0.3)$ & $7,469.7$ & -3.30 & 93.7 & 0.40 & 0.35 \\
\hline $\mathrm{U}^{*}(\beta=-3)$ & $7,529.3$ & -8.11 & 104.9 & 0.71 & 0.51 \\
\hline $\mathrm{U}^{*}(\beta=3)$ & $7,582.5$ & -8.57 & 107.3 & 0.70 & 0.54 \\
\hline
\end{tabular}

The results outlined above indicate that the market operator may want to introduce incentives to avoid large deviations between DA bid and RT delivery. We now repeat the analysis with a \$5/MWh deviation penalty. The results are shown in Fig. 7-21 and Fig. 7-22. In this case, it is actually the point forecast that performs the best in terms of total profit. Among the strategies considering the price predictions, it is the risk-averse strategies that perform better (and in particular, the CVAR ones), both in terms of total profit and CVAR. The poorer performance of the profit-based and risk-prone strategies are probably caused by inaccuracies of the price forecasts. When the price predictions indicate a large different between DA and RT prices, the optimal strategy will be to deviate from the wind power forecast. However, if the price difference does not materialize, the wind farm will be penalized by the deviation penalty. In this case, it therefore appears to be better to rely on the wind power forecast without considering the price predictions. ${ }^{30}$ Furthermore, the interests of the wind farm owner and the system operator are well aligned in this case (Fig. 7-22 ). The strategy that does not bid into the DA market (zero) still gives a low risk, but the total profit is much smaller than for the other strategies. The results with a $\$ 5 / \mathrm{MWh}$ deviation penalty are summarized in Table 7-8. Note that with a deviation penalty there is less difference between the strategies in terms of the average DA bid.

\footnotetext{
${ }^{30}$ Of course, the price predictions used in this study are somewhat rudimentary, simply assuming that prices follow a normal distribution based on historical prices. However, the price predictions could easily be replaced with more advanced price forecasting models. The only requirement is that the price distribution needs to be represented as a discrete set of price realizations for each individual hour.
} 


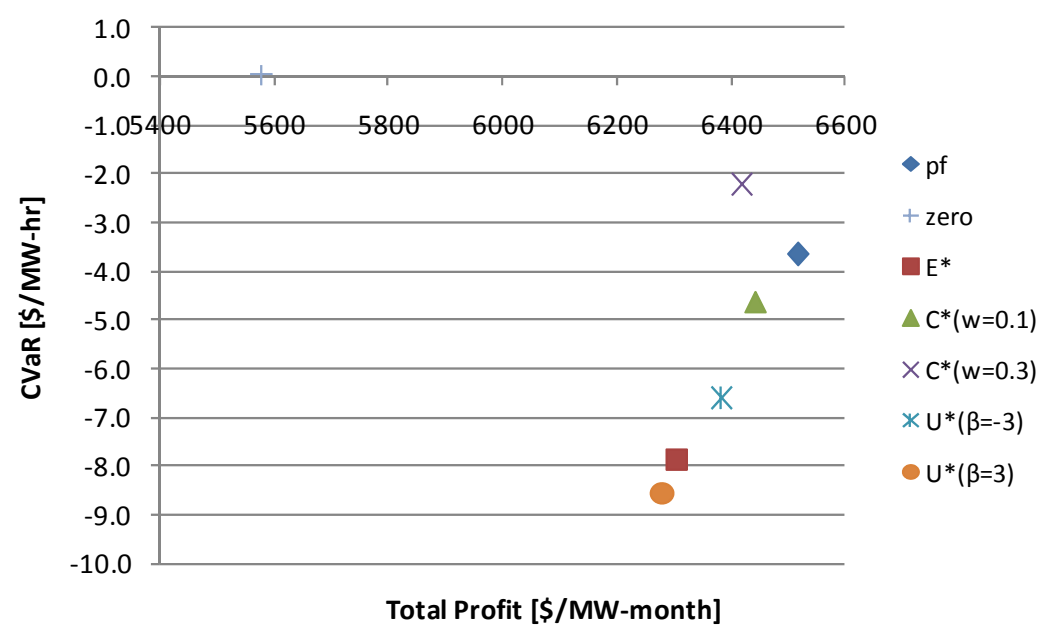

Fig. 7-21 Total monthly profit vs. hourly conditional value at risk, $\$ 5 / \mathrm{MWh}$ deviation penalty.

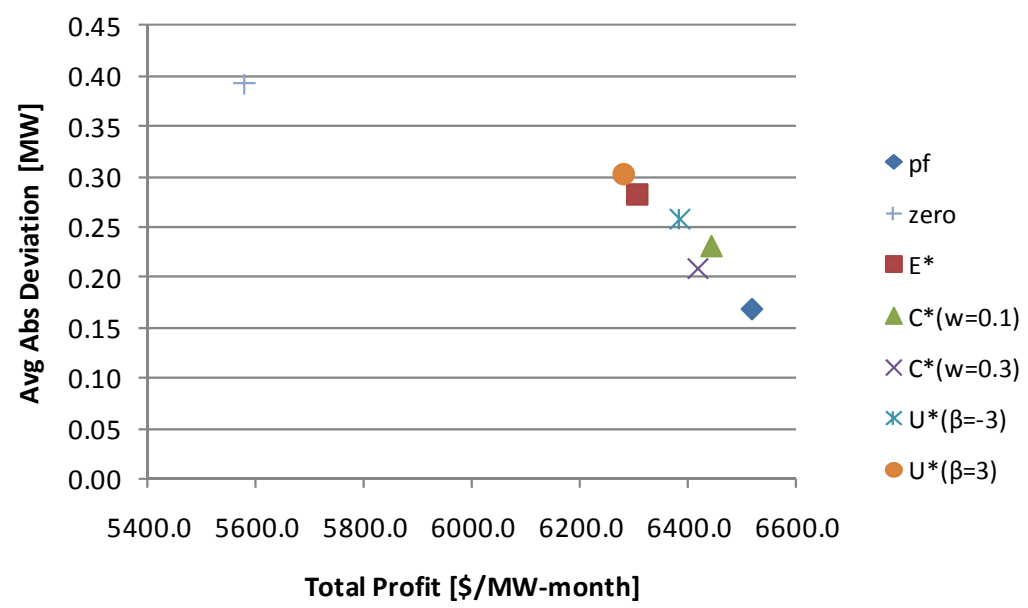

Fig. 7-22 October profit vs. average absolute deviation from schedule, $\$ 5 / \mathrm{MWh}$ deviation penalty.

Table 7-7 Results summary for October with different decision criteria, $\$ 5 / \mathrm{MWh}$ deviation penalty.

\begin{tabular}{|c|c|c|c|c|c|}
\hline Criteria & $\begin{array}{c}\text { Total Profit } \\
{[\$]}\end{array}$ & $\begin{array}{l}\text { CVAR } \\
{[\$ / \mathrm{hr}]}\end{array}$ & $\begin{array}{c}\text { Variance } \\
{\left[\$^{2} / \mathrm{hr}\right]}\end{array}$ & Avg. Bid & $\begin{array}{c}\text { Avg. } \\
\text { Deviation }\end{array}$ \\
\hline $\mathrm{pf}$ & $6,517.7$ & -3.64 & 76.1 & 0.38 & 0.17 \\
\hline zero & $5,578.0$ & -0.62 & 58.6 & 0.00 & 0.39 \\
\hline $\mathrm{E}^{*}$ & $6,304.3$ & -7.88 & 98.4 & 0.50 & 0.28 \\
\hline$C^{*}(w=0.1)$ & $6,442.9$ & -4.65 & 85.2 & 0.39 & 0.23 \\
\hline$C^{*}(w=0.3)$ & $6,417.7$ & -2.22 & 73.2 & 0.28 & 0.21 \\
\hline $\mathrm{U}^{*}(\beta=-3)$ & $6,382.9$ & -6.61 & 92.6 & 0.48 & 0.26 \\
\hline $\mathrm{U}^{*}(\beta=3)$ & $6,279.3$ & -8.58 & 101.2 & 0.53 & 0.30 \\
\hline
\end{tabular}


So far, we have considered WPF uncertainty as a set of scenarios in the DA bidding problem. However, one could also use the forecast quantiles directly to decide on how much to bid into the DA market. In fact, it was shown in [23] that for a risk-neutral wind power producer that always faces a financial loss from deviations, the optimal schedule is the $\mathrm{c}^{+} /\left(\mathrm{c}^{+}+\mathrm{c}^{-}\right)$quantile, where $\mathrm{c}^{+}, \mathrm{c}^{-}$ are the cost of positive and negative deviations. These assumptions do not hold true in U.S. LMP-based markets, as discussed in Section 7.3.

Fig. 7-23 shows the monthly profit for different quantile bids. When there is no deviation penalty, the wind power producer's profit increases by bidding a higher quantile, and the $90 \%$ quantile gives the highest profit (this corresponds to overscheduling, as there is a $90 \%$ chance that the actual generation will be below this quantile). However, the bidding strategy based on the expected profit criterion $\left(\mathrm{E}^{*}\right)$ gives a considerably higher profit. When a deviation penalty is introduced, a bid according to $60 \%$ quantile produces a slightly higher profit than the point forecast, whereas bids according to $\mathrm{E}^{*}$ gives a lower profit in this case. As a reference, optimal bidding with perfect information on prices and wind power would produce a total monthly profit of $\$ 9,740$ and $\$ 8,405$, respectively, in the cases with and without a deviation penalty. Bidding the realized wind generation would yield $\$ 7,255$ in both cases. Fig. 7-24 shows that the CVAR decreases for higher quantile bids, which means that the risk level increases as more capacity is offered into the DA market.
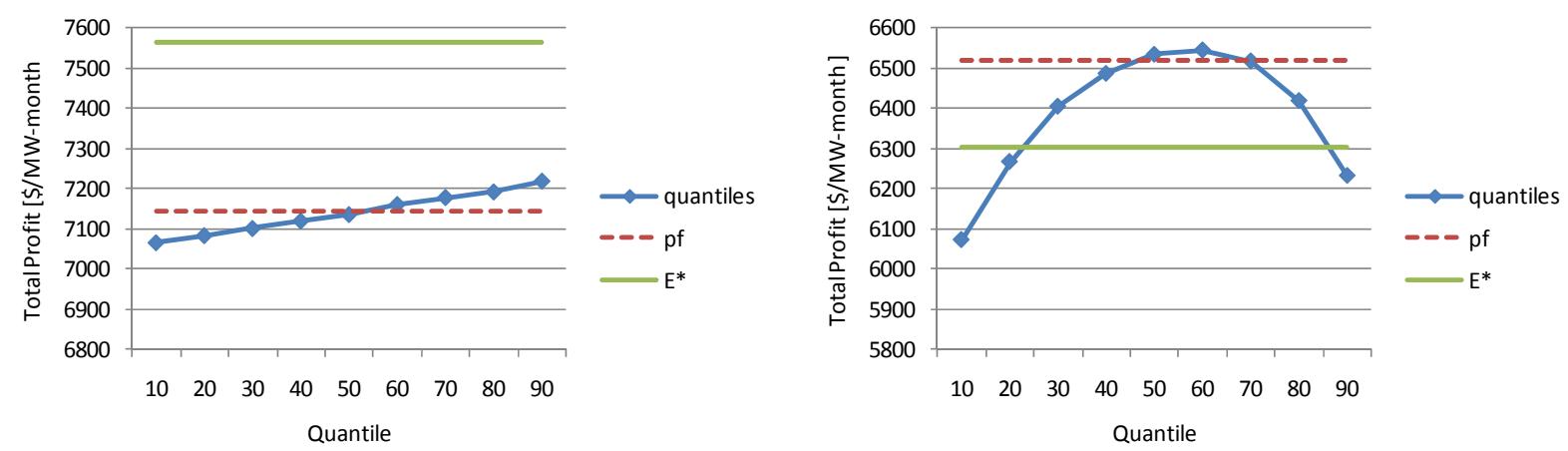

Fig. 7-23 Monthly profit for different quantile forecasts compared to bidding based on point forecast and based on the expected profit criterion, no penalty (left), $\$ 5 / \mathrm{MWh}$ deviation penalty (right).
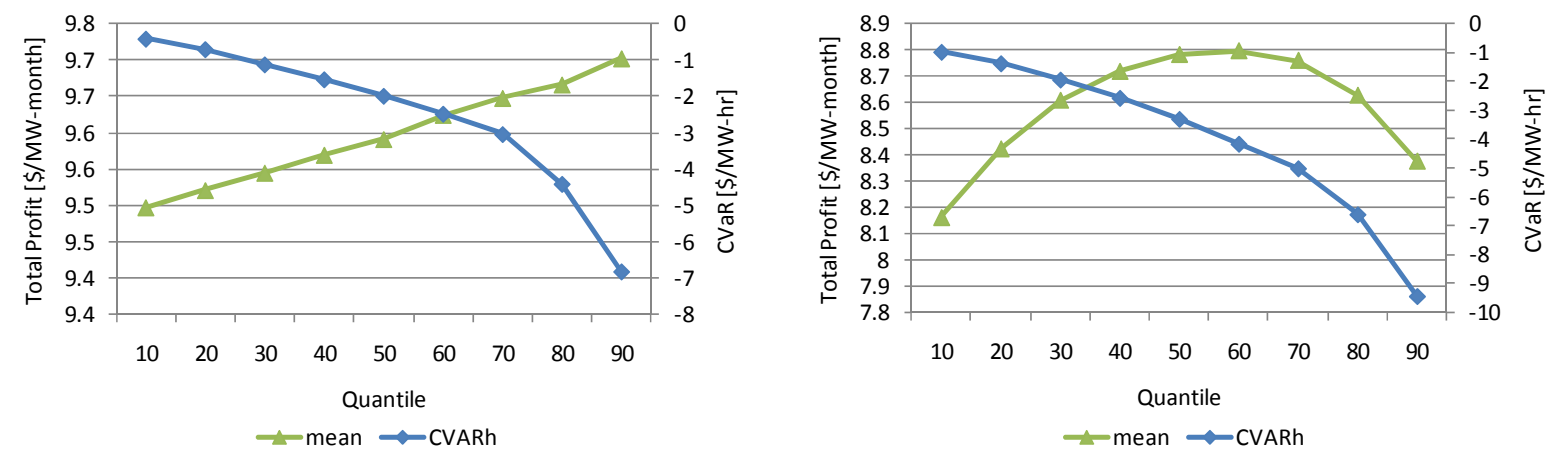

Fig. 7-24 Average hourly profit and CVAR for different quantile forecasts, no penalty (left), $\$ 5 / \mathrm{MWh}$ deviation penalty (right). 
Finally, we test the use of different methods to estimate the probabilistic wind power forecast. In the results presented above, the probabilistic forecasts were based on quantile regression, which is a relatively common approach to statistically estimate WPF uncertainty. However, in this project, we have developed two novel methods for probabilistic WPF with kernel density estimation (KDE), namely based on the Nadaraya-Watson (NW) [93] and quantile copula (QC) [94] [95] estimators. Results from [93], [94], and [95] indicate that the KDE methods produce better probabilistic forecasts than quantile regression $(\mathrm{QR})$ in terms of calibration, which is a measure for how well the forecasted quantiles match the distribution of realized wind power generation. We find the same results when comparing different probabilistic forecasting methods on the wind farm data for this case study. Fig. 7-25 shows that the deviation from perfect calibration is smaller for the KDF-based NW and QC methods compared to linear and splines QR. When it comes to sharpness, which is a measure of the width of the confidence intervals of the probabilistic forecasts, there are only small differences between the four methods.
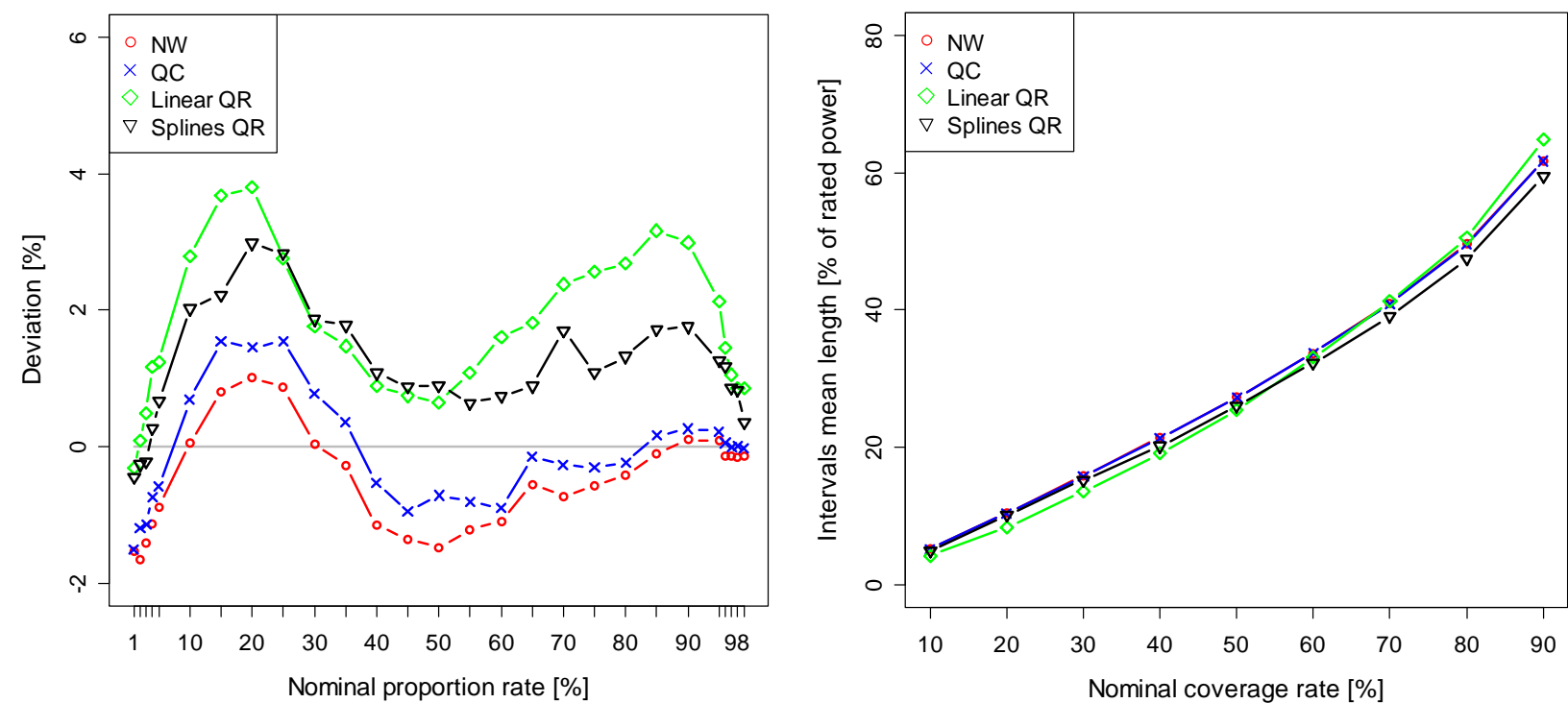

Fig. 7-25 Evaluation of probabilistic forecasts for different methods: calibration (left) and sharpness (right). Training period: January-July 2006; test period: August-December 2006.

In order to investigate the impact of the different forecasting methods, we repeat the monthly runs using the NW and QC methods for probabilistic WPF and for the CVAR strategy under a $\$ 5 / \mathrm{MWh}$ deviation penalty. The results are compared to the original run with probabilistic WPF from QR and scenario generation. Table 7-8 shows that the choice of probabilistic wind power forecast apparently has only limited impact on the results in this case. For the current assumptions, the differences between the WPF methods are small for the CVAR bidding strategy. The differences between the various bidding strategies, which are driven largely by the price projections, are much larger, as we have seen above. However, further work is needed to investigate how the decisions are influenced by the probabilistic WPF methods. Our initial results from a real-world wind farm indicate that the choice of forecasting method has a larger impact on the results. 
Table 7-8 Summary of results for October with the $C^{*}(w=0.1)$ strategy for different wind power uncertainty forecasts, $\$ 5 / \mathrm{MWh}$ deviation penalty.

\begin{tabular}{|l|c|c|c|c|c|}
\hline Criteria & $\begin{array}{c}\text { Total Profit } \\
{[\$]}\end{array}$ & $\begin{array}{c}\text { CVAR } \\
{[\$ / \mathrm{hr}]}\end{array}$ & $\begin{array}{c}\text { Variance } \\
{\left[\${ }^{2} / \mathrm{hr}\right]}\end{array}$ & Avg. Bid & Avg. Deviation \\
\hline $\begin{array}{l}\text { QR } \\
\text { scenario }\end{array}$ & $6,442.9$ & -4.65 & 85.2 & 0.395 & 0.231 \\
\hline QC & $6,433.5$ & -4.74 & 85.3 & 0.401 & 0.234 \\
\hline NW & $6,433.2$ & -4.70 & 85.1 & 0.401 & 0.235 \\
\hline
\end{tabular}

\subsection{Conclusion}

Improved scheduling decisions and better risk management will increase the viability of wind power in the long run. We conclude that stochastic models, like the one proposed here, will be important tools to control the trade-off between risk and return for wind power producers participating in electricity markets. The results presented in this chapter demonstrate that probabilistic wind power forecasts can play an important role in guiding wind power trading decisions in the DA and RT markets. The results also indicate that price forecasts are important in order to derive good trading strategies, particularly under market designs without penalties for deviations between DA schedule and RT delivery. Furthermore, risk preferences clearly influence the optimal trading decisions, with risk-averse owners bidding less energy into the DA market to reduce the exposure to high RT prices. The decisions are also highly dependent on market design, and the results show that a deviation penalty drives the optimal DA bid closer to the point forecast for all strategies. This is in the interest of the system operator, as it makes the task of balancing supply and demand in the system an easier one. However, deviation penalties also add a significant financial burden on wind power producers and therefore reduce the profitability of wind power. Revising market rules to give efficient operational incentives without adding an undue burden on wind power and other renewables will clearly be a challenge as we move forward.

The wind power trading model and analysis outlined in this chapter are general in nature and can be extended in several directions. For instance, the model could benefit from more advanced price predictions, which capture potential relationships between wind power and prices. Further exploration of different probabilistic WPF methods and their impact on trading decisions should also be investigated. Moreover, alternative market rules (different deviation penalties and other incentive schemes, intra-day markets, etc.) can be introduced for a more detailed analysis of the impact of market design on bidding decisions, wind power profits, and the overall operation of the power system. Considering the interplay between different assets, including wind power, in a portfolio is also an interesting extension. Overall, more testing on real-world wind data would contribute to validate the methodology and document the advantages of the proposed modeling framework. We are addressing some of these issues in our recent work, which is documented in [102]. 


\section{CONCLUSIONS}

This report presented our work on the application of advanced WPF in operational decisions, from the perspectives of both system operators and wind power producers. The main findings and conclusions are summarized below.

Different agents in the electricity market may disagree when it comes to assessing a wind power forecast according to its value and quality. Our case study of the Iberian electricity market showed that a "neutral" model (in the forecaster's paradigm sense) might not lead to maximized profits for wind power producers. In fact, if imbalance prices are asymmetric, the profitmaximizing bid will deviate from the unbiased point forecast. This means that a wind power producer may be willing to reduce the forecasts' accuracy in exchange for an increase in income. Yet, electricity markets should provide the participants with incentives for delivering their unbiased forecast information through their scheduling and bidding. Our contributions regarding this topic include:

- A challenge to the assumption of the mathematical neutrality of the forecasting models;

- A thorough discussion of conflicting interests associated with the "goodness" of forecasts for wind power producers and system operators;

- A comparison of the relative advantages and drawbacks of several state-of-the art criteria used in the training of statistical WPF models;

- A new approach proposed to achieve acceptable "compromise" solutions to market participants in the choice of a forecasting model with the parametric correntropy criterion from information theoretic learning; and

- The verification of the proposed theoretical concepts in a representative case study of the Iberian electricity market (Spain and Portugal).

WPF can clearly contribute to a more efficient integration of wind power and other renewable resources into the electric power grid. In a review of the current use of WPF in five ISO/RTO markets in the United States, we found that most system operators are already using WPF to support operational decisions; however, the experience to date is still relatively limited. There is therefore a need to better integrate WPF into operational procedures, including determination of operating reserve requirements, DA market clearing, reliability commitment decisions, and RT dispatch. Improving the efficiency and reliability of power systems with high shares of wind power can be achieved through:

- Developing new tools and procedures for system operators and market participants, so as to make more efficient use of WPF information, enabling better operational decisions under the growing uncertainty and variability due to the increasing penetration of renewables;

- $\quad$ Making improvements in general decision making under uncertainty and particularly in the handling of wind power uncertainty in commitment and dispatch of energy and operating reserves; and

- $\quad$ Promoting improvements in WPF models by better tuning them to the specific needs of the forecast users, such as better predictions of uncertainty and ramping events for system operators. 
We have conducted a detailed analysis of the impact of WPF errors on UC and ED. In particular, we have analyzed the potential use of probabilistic WPF in the scheduling and dispatch of power systems with a mix of thermal power plants and wind power. We have developed a stochastic UC model, where wind power uncertainty is represented in terms of a set of forecast scenarios. We have also developed a scenario reduction algorithm to select an adequate set of wind power scenarios for use in stochastic UC. Moreover, we have explored how probabilistic forecasts can be used to derive dynamic operating reserve requirements for use in a deterministic UC. Based on the application of these tools in several case studies, we conclude that:

- Commitment decisions based on a deterministic wind power point forecast are too risky and are likely to result in high levels of unserved load and reserve. Hence, additional operating reserves are needed to handle the uncertainty in wind power.

- For deterministic UC, a dynamic operating reserve requirement based on probabilistic forecasts performs better than a fixed additional reserve, because the amount of additional operating reserves is adjusted according to the predicted forecast uncertainty.

- Stochastic UC with wind power scenarios has advantages over the traditional deterministic scheduling approaches. First, stochastic UC addresses inter-temporal variability through the scenario representation of uncertainty. Second, the expected cost of scarcity is explicitly taken into account in the objective function. However, the high computational burden may prevent implementation on large-scale systems.

- The choice of scenario set is very important in stochastic UC. Formal scenario reduction methods appear to lower the level of hedging in commitment decisions, as they tend to reduce the overall scenario variance. At the same time, a higher number of scenarios improves the operating results, but may be prohibitive for computational reasons. A certain level of additional operating reserves is therefore also likely to be needed with stochastic UC, as the scenarios do not capture the full range of forecast uncertainty.

- Deterministic UC combined with dynamic operating reserves does not address intertemporal ramping events, but the approach is better aligned with current operating practices and the computational burden is much smaller than with stochastic UC.

- Stochastic UC with forecast scenarios and deterministic UC with dynamic operating reserves give similar results in terms of cost and reliability in our case studies, although there may be distinct differences in commitment and dispatch decisions.

- Probabilistic forecasts contribute to improved operational decisions both in the cases of dynamic operating reserves and stochastic UC.

- More work should be conducted to analyze the potential use of probabilistic forecasting and stochastic scheduling under more realistic assumptions, for instance by introducing additional operational constraints (e.g., transmission) in the UC formulation.

- It is important for system and market operators to consider their risk preferences in determining operational strategies, and properly represent this in the mathematical models used to support their decisions.

- We recommend that system operators start making use of probabilistic forecasting information in their scheduling decisions. A first step would be to move toward the use of dynamic operating reserve requirements. Next, as probabilistic WPF and computational resources improve, system operators should consider an entirely stochastic scheduling approach, where WPF uncertainty is fully represented in the UC formulation. 
We also investigated the use of probabilistic WPF for wind power producers. We presented a model for optimal trading of wind power in DA electricity markets under uncertainty in wind power and prices. We argued that such models constitute important tools to control the trade-off between risk and return for wind power producers that participate in the electricity market. Our results have demonstrated that:

- $\quad$ Probabilistic WPF can play an important role in guiding wind power trading decisions in the DA and RT markets;

- $\quad$ Price forecasts are important in order to derive good trading strategies, particularly when there are no deviation penalties between DA schedule and RT delivery;

- $\quad$ Risk preferences influence the optimal trading decisions, with risk-averse wind power producers bidding less energy into the DA market to reduce the exposure to high RT prices;

- Deviation penalties drive the optimal DA bid closer to the point forecast, which implies that system operators have an easier task in balancing supply and demand, but also that wind power producers' income is reduced;

- Improved scheduling decisions and better risk management will increase the viability of wind power in the long run; and

- Overall, an important challenge will be to define market rules that provide efficient operational incentives without altering the burden on wind power and other renewables. 
This page intentionally blank 


\section{REFERENCES}

[1] U.S. Department of Energy (DOE), "20\% Wind Energy by 2030: Increasing Wind Energy's Contribution to U.S. Electricity Supply,” DOE Office of Energy Efficiency and Renewable Energy Report, July 2008. Online: http://wwwl.eere.energy.gov/ windandhydro/wind_2030.html.

[2] European Union (EU), "Climate change: Commission welcomes final adoption of Europe's climate and energy package," Press Release, EU, Dec. 17, 2008. Online: http://europa.eu/rapid/pressReleasesAction.do?reference=IP/08/1998.

[3] Global Wind Energy Council (GWEC), "Global Wind Report: Annual Market Update 2010," GWEC Report, April 2011.

[4] C. Monteiro, R. Bessa, V. Miranda, A. Botterud, J. Wang, G. Conzelmann, "Wind Power Forecasting: State-of-the-art 2009," Technical report ANL/DIS-10-1. Argonne National Laboratory, 2009. Online: http://www.dis.anl.gov/projects/windpowerforecasting.html.

[5] J. Mendes, R.J. Bessa, H. Keko, J. Sumaili, V. Miranda, A. Botterud, Z. Zhou, J. Wang, "Development and Testing of Improved Wind Power Forecasting Methods," Technical report, INECS Porto and Argonne National Laboratory, Sept. 2011.

[6] A. Costa, A. Crespo, J. Navarro, G. Lizcano, H. Madsen, E. Feitosa, "A review on the young history of the wind power short-term prediction," Renewable and Sustainable Energy Reviews, Vol. 12, pp. 1725-1744, 2008.

[7] B. Ernst, B. Oakleaf, M.L. Ahlstrom, M. Lange, C. Moehrlen, B. Lange, U. Focken, K. Rohrig, "Predicting the wind," IEEE Power \& Energy Magazine, Vol. 5, No. 6, pp. 78-89, 2007.

[8] H. Madsen, P. Pinson, H. Kariniotakis, H.A. Nielsen, T.S. Nielsen, "Standardizing the performance evaluation of short-term wind prediction models," Wind Engineering, Vol. 29, pp. 475-489, 2005.

[9] Industry Work Group, "Wind power forecasting pilot project - industry work group report,” Technical Report, 2008. Online: http://www.aeso.ca/gridoperations/13825.html.

[10] A.H. Murphy, "What is a good forecast? An essay on the nature of goodness in weather forecasting," Weather and Forecasting, Vol. 8, pp. 281-293, 1993.

[11] A.H. Murphy, R.L. Winkler, "A general framework for forecast verification,” Monthly Weather Review, Vol. 115, pp. 1330-1338, 1987.

[12] P. Pinson, "Estimation of the uncertainty in wind power forecasting," PhD Thesis, Ecole des Mines de Paris, Paris, France, 2006. 
[13] T.R. Stewart, R.W. Katz, A.H. Murphy, "Value of weather information: a descriptive study of the fruit-frost problem," Bulletin of the American Meteorological Society, Vol. 65, pp. 126-137, 1984.

[14] J. Usaola, O. Ravelo, G. González, F. Soto, M.C. Dávila, B. Díaz-Guerra, "Benefits for wind energy in electricity markets from using short term wind power prediction tools; a simulation study," Wind Engineering, Vol. 28, pp. 119-127, 2004.

[15] R.J. Barthelmie, F. Murray, S.C. Pryor, "The economic benefit of short-term forecasting for wind energy in the UK electricity market," Energy Policy, Vol. 36, pp. 1687-1696, 2008.

[16] J.L. Angarita-Márqueza, C.A. Hernandez-Aramburo, J. Usaola, "Analysis of a wind farm's revenue in the British and Spanish markets," Energy Policy, Vol. 35, pp. 50515059, 2007.

[17] P. Pinson, C. Chevallier, G. Kariniotakis, "Trading wind generation with short-term probabilistic forecasts of wind power," IEEE Transactions on Power Systems, Vol. 22, No. 3, pp. 1148-1156, 2007.

[18] A. Fabbri, T. Gomez, J.R. Abbad, V.H. Quezada, "Assessment of the Cost Associated with Wind Generation Prediction Errors in a Liberalized Electricity Market," IEEE Transactions on Power Systems, Vol. 20, No. 3, pp. 1440-1446, 2005.

[19] J. Matevosyan, L. Söder, "Minimization of Imbalance Costs Trading Wind Power on the Short-Term Power Market," IEEE Transactions on Power Systems, Vol. 21, No. 3, pp. 1396-1404, 2006.

[20] F. Bourry, J. Juban, L.M. Costa, G. Kariniotakis, "Advanced strategies for wind power trading in short-term electricity markets," Proceedings European Wind Energy Conference \& Exhibition EWEC 08, Brussels, Belgium, March 31-April 3, 2008.

[21] U. Linnet, "Tools supporting wind energy trade in deregulated markets," MSc Thesis, Technical University of Denmark, Lyngby, Denmark, July 2005.

[22] R.W. Katz, A.H. Murphy, "Quality/value relationships for imperfect weather forecasts in a prototype multistage decision-making model," Journal of Forecasting, Vol. 9, pp. 7586, 2006.

[23] J.B. Bremnes, "Probabilistic Wind Power Forecasts Using Local Quantile Regression," Wind Energy, Vol. 7, pp. 47-54, 2004.

[24] A. Botterud, J. Wang, R.J. Bessa, H. Keko, V. Miranda, "Risk Management and Optimal Bidding for a Wind Power Producer," Proceedings IEEE PES General Meeting, Minneapolis, Minn., July 2010. 
[25] H.F. Ravn, "Short term wind power prognosis with different success criteria," Proceedings International Conference on Probabilistic Methods Applied to Power Systems - PMAPS 2006, Stockholm, Sweden, June, 2006.

[26] A. Botterud, J. Wang, V. Miranda, R.J. Bessa, "Wind Power Forecasting in U.S. Electricity Markets," Electricity Journal, Vol. 23, No. 3, pp. 71-82, 2010.

[27] K. Verhaegen, L. Meeus, R. Belmans, "Development of balancing in the internal electricity market in Europe," Proceedings the European Wind Energy Conference \& Exhibition EWEC 06, Athens, Greece, 2006.

[28] Y. Rebours, D.S. Kirschen, "A survey of definitions and specifications of reserve services," Technical Report, University of Manchester, Oct. 2005.

[29] R. Doherty, M. O’Malley, "A New Approach to Quantify Reserve Demand in Systems with Significant Installed Wind Capacity," IEEE Transactions on Power Systems, Vol. 20, No. 2, pp. 587-595, 2005.

[30] M.A. Matos, R. Bessa, "Operating Reserve Adequacy Evaluation using Uncertainties of Wind Power Forecast," Proceedings IEEE PowerTech Conference, Bucharest, Romania, 2009.

[31] H. Holttinen, "Impact of hourly wind power variations on the system operation in the Nordic countries,” Wind Energy, Vol. 8, pp. 197-218, 2004.

[32] R. Doherty, E. Denny, M. O’Malley, "System Operation with a Significant Wind Power Penetration," Proceedings IEEE Power Engineering Society General Meeting, Denver, Colo., USA, June 2004.

[33] M.D. Ilic, Y. Makarov, D. Hawkins, "Operations of Electric Power Systems with High Penetration of Wind Power: Risks and Possible Solutions," Proceedings IEEE Power Engineering Society General Meeting, Tampa, Florida, USA, June 2007.

[34] UCTE NetWork of experts on Wind Power, "Wind power in the UCTE interconnected system," UCTE Position Paper, 2004.

[35] E. Van Damme, "Stability and perfection of Nash equilibria," Springer-Verlag, Berlin, 1991.

[36] P. Faratin, C. Sierra, N.R. Jennings, "Using similarity criteria to make issue trade-offs in automated negotiations," Artificial Intelligence, Vol. 142, pp. 205-237, 2002.

[37] R. Bessa, V. Miranda, J. Gama, "Entropy and Correntropy against Minimum Square Error in Off-line and On-line 3-Day Ahead Wind Power Forecasting," IEEE Transactions on Power Systems, Vol. 24, No. 4, pp. 1657-1666, 2009.

[38] J.C. Principe, D. Xu, J. Fisher, "Information theoretic learning," in Unsupervised Adaptive Filtering, Simon Haykin (Editor), Wiley, pp. 265-319, 2000. 
[39] D. Erdogmus, J.C. Principe, "Generalized Information Potential Criterion for Adaptive System Training," IEEE Transactions on Neural Networks, Vol. 13, No. 5, pp. 10351044, 2002.

[40] W. Liu, P. Pokharel, J.C. Principe, "Correntropy: Properties and Applications in NonGaussian Signal Processing," IEEE Transactions on Signal Processing, Vol. 55, No. 11, pp. 5286-5298, 2007.

[41] J. Xu, "Nonlinear signal processing based on reproducing Kernel Hilbert space," $\mathrm{PhD}$ Thesis, University of Florida, Gainesville, Florida, USA, 2007.

[42] H. Bludszuweit, "Reduction of the uncertainty of wind power predictions using energy storage," PhD Thesis, Universidad de Zaragoza, Spain, 2009.

[43] North American Electric Reliability Council (NERC), "Accommodating High Levels of Variable Generation,” NERC Special Report, April 2009.

[44] U.S. Department of Energy (DOE), "20\% Wind Energy by 2030: Increasing Wind Energy's Contribution to U.S. Electricity Supply,” DOE Report, July 2008.

[45] W. Grant, D. Edelson, J. Dumas, J. Zack, M. Ahlstrom, J. Kehler, P. Storck, J. Lerner, K. Parks, C. Finley, "Change in the Air: Operational Challenges in Wind-Power Production and Prediction," IEEE Power \& Energy Magazine, Vol. 7, No. 6, pp. 47-58, 2009.

[46] M. Ahlstrom, L. Jones, R. Zavadil, W. Grant, "The Future of Wind Forecasting and Utility Operations: Planning for Improved System Operations," IEEE Power \& Energy Magazine, Vol. 3, No. 6, pp. 57-64, 2005.

[47] North American Reliability Council (NERC), "Reliability Standards for the Bulk Electric System of North America," NERC, April 2009. Online: http://www.nerc.com/page.php?cid=2|20.

[48] M.A. Ortega-Vazquez, D.S. Kirschen, "Estimating the Spinning Reserve Requirements in Systems With Significant Wind Power Generation Penetration," IEEE Transactions on Power Systems, Vol. 24, No. 1, pp. 114-124, 2009.

[49] W.W. Hogan, “A Model for a Zonal Operating Reserve Demand Curve," MIT Operations Research Seminar, October 15, 2009. Online: http://www.whogan.com/.

[50] Electricity Reliability Council of Texas (ERCOT), "ERCOT Methodologies for Determining Ancillary Service Requirements," ERCOT, December 2009. Online: http://www.ercot.com/mktinfo/services/.

[51] General Electric (GE), "Analysis of Wind Generation Impact on ERCOT Ancillary Services Requirements,” GE Report, March 28, $2008 . \quad$ Online: http://www.uwig.org/opimpactsdocs.html. 
[52] R. Wiser, M. Bolinger, "2008 Wind Technologies Market Report," Lawrence Berkeley National Laboratory, July 2009. Online: http://eetd.lbl.gov/EA/EMS/reports/2008-windtechnologies.pdf.

[53] R. Sioshansi, D. Hurlbut, "Market protocols in ERCOT and their effect on wind generation," Energy Policy, Vol. 38, No. 7, pp. 3192-3197, 2010.

[54] D. Maggio, "Integrating Wind Forecasting into Market Operation - ERCOT," Presentation Wind Forecasting Workshop, Utility Wind Integration Group (UWIG), Phoenix, AZ, February 2009.

[55] R. Barth, H. Brand, P. Meibom, C. Weber, "A Stochastic Unit-commitment model for the Evaluation of the Impacts of Integration of Large Amounts of Intermittent Wind Power," Proceedings 9th Int. Conf. on Probabilistic Methods Applied to Power Systems, Stockholm, Sweden, 2006.

[56] F. Bouffard, F. Galiana, "Stochastic Security for Operations Planning with Significant Wind Power Generation," IEEE Transactions on Power Systems, Vol. 23, No. 2, pp. 306-316, 2008.

[57] J. Wang, M. Shahidehpour, Z. Li, "Security-Constrained Unit Commitment with Volatile Wind Power Generation," IEEE Transactions on Power Systems, Vol. 23, No. 3, pp. 1319-1327, 2008.

[58] P.A. Ruiz, C.R. Philbrick, P.W. Sauer, "Wind Power Day-Ahead Uncertainty Management through Stochastic Unit Commitment Policies," Proceedings Power Systems Conference and Exhibition, Seattle, March 2009.

[59] A. Tuohy, P. Meibom, E. Denny, M. O'Malley, "Unit Commitment for Systems With Significant Wind Penetration," IEEE Transactions on Power Systems, Vol. 24, No. 2, pp. 592-601, 2009.

[60] J. Wang, A. Botterud, G. Conzelmann, V. Miranda, C. Monteiro, G. Sheble, "Impact of Wind Power Forecasting on Unit Commitment and Dispatch," 8th Int. Workshop on Wind: Large-Scale Integration of Wind Power into Power Systems, Bremen, Germany, October 2009.

[61] J. Usaola, "Probabilistic load flow in systems with wind generation," IET Generation, Transmission and Distribution. Vol. 3, No. 12, pp. 1031-1041, 2009.

[62] R. Gonzales, R. Mukerji, M. Swider, D. Allen, R. Pike, D. Edelson, F. Nelson, J. Adams, Integration of Wind into System Dispatch, New York ISO White Paper, Oct. 2008. Online: http://www.nyiso.com/public/webdocs/documents/white_papers/wind_ manage ment_whitepaper_11202008.pdf.

[63] Utility Wind Integration Group (UWIG), "Wind Power and Electricity Markets," UWIG Report, August 2009. Online: http://www.uwig.org/. 
[64] Utility Wind Integration Group (UWIG), "Central Wind Power Forecasting Programs in North America by Regional Transmission Organizations and Electric Utilities," UWIG Report, December 2009. Online: http://www.uwig.org/.

[65] N.P. Padhy, "Unit Commitment-A Bibliographical Survey," IEEE Transactions on Power Systems, Vol. 19, No. 2, pp. 1196-1205, 2004.

[66] Wind Power Integration in Liberalised Electricity Markets (Wilmar) Project. Online: http://www.wilmar.risoe.dk.

[67] A. Tuohy, E. Denny, M. O’Malley, "Rolling Unit Commitment for Systems with Significant Installed Wind Capacity," Proceedings 2007 IEEE Lausanne PowerTech, pp. 1380-1385, July 1-5, 2007.

[68] A. Tuohy, P. Meibom, M. O’Malley, "Benefits of Stochastic Scheduling for Power Systems with Significant Installed Wind Power," Proceedings $10^{\text {th }}$ Int. Conference on Probabilistic Methods Applied to Power Systems (PMAPS), Mayagüez, Puerto Rico, June 2008.

[69] B.C. Ummels, M. Gibescu, E. Pelgrum, W.L. Kling, A.J. Brand, "Impacts of Wind Power on Thermal Generation Unit Commitment and Dispatch," IEEE Transactions on Energy Conversion, Vol. 22, No. 1, pp. 44-51, 2007.

[70] P.A. Ruiz, C.R. Philbrick, E. Zak, K.W. Cheung, P.W. Sauer, "Uncertainty Management in the Unit Commitment Problem," IEEE Transactions on Power Systems, Vol. 24, No. 2, pp. 642-51, 2009.

[71] E. Delarue, W. D'haeseleer, "Adaptive mixed-integer programming unit commitment strategy for determining the value of forecasting," Applied Energy, Vol. 85, No. 4, pp. 171-181, 2008.

[72] N. Amjady, F. Keynia, "A new spinning reserve requirement forecast method for deregulated electricity markets," Applied Energy, Vol. 87, No. 6, pp. 1870-1879, 2010.

[73] P.J. Luickx, E.D. Delarue, W. D'haeseleer, "Considerations on the backup of wind power: Operational backup,” Applied Energy, Vol. 85, No. 9, pp. 787-799, 2008.

[74] M. Matos, et al. "Probabilistic evaluation of reserve requirements of generating systems with renewable power sources: The Portuguese and Spanish cases," International Journal of Electrical Power \& Energy Systems, Vol. 31, No. 9, pp. 562-569, 2009.

[75] M.A. Matos, R. Bessa, "Setting the Operating Reserve Using Probabilistic Wind Power Forecasts," IEEE Transactions on Power Systems, Vol. 26, No. 2, pp. 594-603, May 2011.

[76] E. Ela, B. Kirby, E. Lannoye, M. Milligan, D. Flynn, B. Zavadil, M. O’Malley, "Evolution of Operating Reserve Determination in Wind Power Integration Studies," 
Proceedings IEEE Power and Energy Society General Meeting, Minneapolis, Minn., July 2010.

[77] H.A. Nielsen, H. Madsen, T.S. Nielsen, "Using Quantile Regression to Extend an Existing Wind Power Forecasting System with Probabilistic Forecasts," Wind Energy, Vol. 9, No. 1-2, pp. 95-108, 2006.

[78] P. Pinson, H. Madsen. H.A. Nielsen, G. Papaefthymiou, B. Klöck1, "From Probabilistic Forecasts to Statistical Scenarios of Short-term Wind Power Production," Wind Energy Vol. 12, No. 1, pp. 51-62, 2009.

[79] M. Carrion, J.M. Arroyo, "A computationally efficient mixed-integer linear formulation for the thermal unit commitment problem," IEEE Transactions on Power Systems, Vol. 21, No. 3, pp. 1371-8, 2006.

[80] Midwest ISO (MISO), "Energy and Operating Reserve Markets Business Practices Manual.” Online: http://www.midwestiso.org.

[81] S.A. Kazarlis, A.G. Bakirtzis, V. Petridis, "A Genetic Algorithm Solution to the Unit Commitment Problem," IEEE Transactions on Power Systems, Vol. 11, No. 1, pp. 83-92, 1996.

[82] MISO, "Business Practices Manual - Energy and Operating Reserve Markets: Attachment B Day-Ahead Energy and Operating Reserve Market Software Formulations and Business Logic,” Jan. 2009. Online: http://www.midwestiso.org.

[83] Eastern Wind Integration and Transmission Study (EWITS), EnerNex Corporation. Online: http://www.nrel.gov/wind/systemsintegration/ewits.html.

[84] M. Brower, "Development of Eastern Regional Wind Resource and Wind Plant Output Datasets," National Renewable Energy Laboratory, Golden, Co, Subcontract Report No.: NREL/SR-550-46764, Dec. 2009.

[85] Electricity Reliability Council of Texas (ERCOT). "ERCOT Methodologies for Determining Ancillary Service Requirements," Dec. 2009. Online: http://www.ercot.com/mktinfo/services/.

[86] S. Rao, Weifeng Liu, J. Principe, A. de Medeiros Martins, "Information Theoretic Mean Shift Algorithm," Proceedings of the 2006 16th IEEE Signal Processing Society Workshop on Machine Learning for Signal Processing, 2006, pp. 155-160, 2006.

[87] S. Rao, A. de Medeiros Martins, J.C. Príncipe, "Mean shift: An information theoretic perspective," Pattern Recognition Letters, Vol. 30, No. 3, pp. 222-230, Feb. 2009.

[88] J. Dupačová, N. Gröwe-Kuska, W. Römisch, "Scenario Reduction in Stochastic Programming: An Approach Using Probability Metrics," Mathematical Programming, Series A, Vol. 95, pp. 493-511, 2003. 
[89] N. Gröwe-Kuska, H. Heitsch, W. Römisch, "Scenario reduction and scenario tree construction for power management problems," Proceedings 2003 IEEE Bologna Power Tech Conference, Bologna, Italy, June 2003.

[90] T. Hastie, R. Tibshirani, J. Friedman, “The Elements of Statistical Learning: Data Mining, Inference, and Prediction," $2^{\text {nd }}$ Edition, 2009, Springer, New York.

[91] J.B. MacQueen, "Some methods for classification and analysis of multivariate observations," Proc. 5th Berkeley Symposium on Mathematical Statistics and Probability, 1967, Berkeley, University of California Press, 1, pp. 281-297.

[92] D. Rajan, S. Takriti, "Minimum Up/Down Polytopes of the Unit Commitment Problem with Start-up Costs,” Report RC23628, IBM Research, June 2005.

[93] R.J. Bessa, J. Sumaili, V. Miranda, A. Botterud, J. Wang, E. Constantinescu, “Timeadaptive Kernel Density Forecast: A New Method for Wind Power Uncertainty Modeling," Proceedings $17^{\text {th }}$ Power System Computation Conference (PSCC'11), Stockholm, Sweden, Aug. 2011.

[94] R.J. Bessa, J. Mendes, V. Miranda, A. Botterud, J. Wang, Z. Zhou, "Quantile-Copula Density Forecast for Wind Power Uncertainty Modeling," Proceedings IEEE Trondheim PowerTech 2011, Trondheim, Norway, June 2011.

[95] R.J. Bessa, V. Miranda, A. Botterud, Z. Zhou, J. Wang, "Time-Adaptive Quantile-Copula for Wind Power Probabilistic Forecasting," Renewable Energy, in press, Sept. 2011.

[96] R.J. Hyndman, D.M. Bashtannyk, G.K. Grunwald, "Estimating and Visualizing Conditional Densities," Journal of Computational and Graphical Statistics, Vol. 5, No. 4, pp. 315-336, 1996.

[97] J. Sumaili, H. Keko, V. Miranda, A. Botterud, J. Wang, "Clustering-based Wind Power Scenario Reduction Technique," Proceedings $17^{\text {th }}$ Power System Computation Conference (PSCC'11), Stockholm, Sweden, Aug. 2011.

[98] GAMS/SCENRED. Online: http://www.gams.com/docs/document.htm.

[99] K. Hoyland, M. Kaut, S.W. Wallace, "A Heuristic for Moment-Matching Scenario Generation," Computational Applications and Optimization, Vol. 24, pp. 169-185, 2003.

[100] B. Hasche, "Scenario Tree Generation for an Electricity Market Model," Report AP 3.3, Institut für Energiewirtschaft und Rationelle Energieanwendung (IER), Universität Stuttgart, August 2008.

[101] Z. Zhou, A. Botterud, J. Wang, R.J. Bessa, H. Keko, J. Sumaili, V. Miranda, "Application of Probabilistic Wind Power Forecasts in Electricity Markets," Wind Energy, submitted, June 2011. 
[102] A. Botterud, Z. Zhou, J. Wang, R.J. Bessa, H. Keko, J. Sumaili, V. Miranda, "Wind Power Trading under Uncertainty in LMP markets," IEEE Transactions on Power Systems, accepted, Sept. 2011.

[103] R. Wiser, M. Bolinger, "2009 Wind Technologies Market Report," Lawrence Berkeley National Laboratory, August 2010.

[104] G.N. Bathurst, J. Weatherill, G. Strbac, "Trading Wind Generation in Short-Term Energy Markets," IEEE Transactions on Power Systems, Vol. 17, No. 3, pp. 782-789, 2002.

[105] J. Usaola, M.A. Moreno, "Optimal Bidding of Wind Energy in Intraday Markets," Proceedings $6^{\text {th }}$ International Conference on the European Energy Market, Leuven, Belgium, 2009.

[106] J.M. Morales, A.J. Conejo, J. Pérez-Ruiz, "Short-Term Trading for a Wind Power Producer," IEEE Transactions on Power Systems, Vol. 25, No. 1, pp. 554-564, 2010.

[107] C.J. Dent, J.W. Bialek, B.F. Hobbs, "Opportunity Cost Bidding by Wind Generators in Forward Markets: Analytical Results," IEEE Transactions on Power Systems, Vol. 26, No. 3, pp. 1600-1608, 2011.

[108] S. Stoft, "Power System Economics: Designing Markets for Electricity," IEEE Press, Piscataway, N.J., 2002.

[109] R. Gonzales, R. Mukerji, M. Swider, D. Allen, R. Pike, D. Edelson, F. Nelson, J. Adams, "Integration of Wind into System Dispatch," New York ISO White Paper, Oct. 2008.

[110] Federal Energy Regulatory Commission (FERC), "Integration of Variable Resources," Notice of Proposed Rulemaking, Docket No. RM10-11-000, Nov. 18, 2010.

[111] T. Jónsson, "Forecasting of Electricity Prices Accounting for Wind Power Predictions," MSc Thesis, Technical University of Denmark, Lyngby, Denmark, 2008.

[112] R.T. Rockafellar, S. Uryasev, "Optimization of Conditional Value-at-Risk," Journal of Risk, Vol. 2, pp. 21-41, 2000.

[113] R.L. Keeney, H. Raiffa, "Decisions with Multiple Objectives. Preferences and Value Tradeoffs," Cambridge University Press, 1993. 
This page intentionally blank 



\section{Argonne}

Decision and Information Sciences Division

Argonne National Laboratory

9700 South Cass Avenue, Bldg. 221

Argonne, IL 60439-4844

www.anl.gov 